Portland State University

PDXScholar

Dissertations and Theses

Dissertations and Theses

Summer 8-4-2016

\title{
Thermal Decomposition of Electronic Cigarette Liquids
}

Robert Paul Jensen

Portland State University

Follow this and additional works at: https://pdxscholar.library.pdx.edu/open_access_etds

Part of the Chemistry Commons

Let us know how access to this document benefits you.

Recommended Citation

Jensen, Robert Paul, "Thermal Decomposition of Electronic Cigarette Liquids" (2016). Dissertations and Theses. Paper 3081.

https://doi.org/10.15760/etd.3076

This Dissertation is brought to you for free and open access. It has been accepted for inclusion in Dissertations and Theses by an authorized administrator of PDXScholar. Please contact us if we can make this document more accessible: pdxscholar@pdx.edu. 
Thermal Decomposition of Electronic Cigarette Liquids

by

Robert Paul Jensen

A dissertation submitted in partial fulfillment of the requirements for the degree of

Doctor of Philosophy

in

Chemistry

Dissertation Committee:

David H. Peyton, Chair

Jonathan J. Abramson

Dean B. Atkinson

David R. Stuart

Portland State University

2016 
(C) 2016 Robert Paul Jensen 


\begin{abstract}
Electronic cigarette liquid (e-liquid) is a solution of propylene glycol and/or glycerol with varying concentrations of nicotine and flavorants. Inhalation of vaporized e-liquid is a method of nicotine delivery that is growing in popularity and is commonly regarded as safe relative to smoking traditional tobacco products. The thermal decomposition of glycerol and propylene glycol is typical of alcohols and has been investigated, although not exhaustively. In this work, samples of propylene glycol and glycerol were vaporized using an electronic cigarette (e-cigarette) and were analyzed for evidence of decomposition using nuclear magnetic resonance (NMR) spectroscopy. E-cigarettes are shown to degrade glycerol and propylene glycol into a diverse array of oxidation and dehydration products including glyceraldehyde, lactaldehyde, dihydroxyacetone, hydroxyacetone, glycidol, acrolein, propanal, acetone, allyl alcohol, acetic acid, acetaldehyde, formic acid, and formaldehyde. Evidence is presented that the abundance of these decomposition products may depend upon the temperature of the metal heating element but could also depend upon some catalytic aspect of the metal surface. The combination of formaldehyde with alcohols such as glycerol and propylene glycol was explored; hemiformals are stable hemiacetals that can be detected by NMR spectroscopy and are shown to be subject to hydrolysis when diluted in water. Investigations into smoking a glycerol-based tobacco mixture known as shisha using charcoals instead of metal heating elements also resulted in the dehydration of glycerol and sugars.
\end{abstract}




\section{Dedication}

To my friend David Peyton, who taught me to compromise.

Omnia probate: quod bonum est tenete. 


\section{Acknowledgments}

My love, Natasja Swartz, who illuminates my life.

My family, who endure me at my worst, yet love and support me without fail.

My advisor David Peyton, a patient mentor with a golden soul.

My dear friend Katherine Liebman, the finest chemist I have ever met, who makes the laboratory a wonderful place to work.

My comrade Chase Reinhart, who has a tyrannosaur heart pumping coffee through his noble veins

My labmates Cheryl Shirley, Anna Duell, Alyssa Palmer, Elias Sibhatu, Kelsie Kendrick, Kevin Murphy, Westin Morril, Steven Burgess, Rolf Winter, and Troy Wahl.

The distinguished gentlemen of my dissertation committee, Dean Atkinson, Dave Stuart, and Jon Abramson.

My friend Niles Lehman, the most curious and interesting person with whom I have ever conversed.

My friends and colleagues Sara Rosario, Alan Cornelius, Nicholas Day, Capri Price, Kathryn Hosbein, Kelly Chacon, Adriana Bon, Aaron Rogers, Thomas Seidl, Eric Young, Jessica Yeates, Lei Wang, Keith James, Davin Rautiola, Kudzanai Chipiso, Katherine Payne, Annah Farashishiko, James Proestos, Sheng-Kuei Chiu, Anna Brown, Brian Larson, Patience Marimo, Joe Harworth, and many others.

My instructors and advisors JoAnn Peters, Dion Rivera, Dirk Iwata-Reuyl, Mark Woods, Steve Benight, Levente Fabry-Asztalos, Dave Reingold, and many others. My collaborators Robert Strongin, Jorge Escobedo, Wentai Luo, and Jim Pankow. My friend Dave Cordes, a chemist after my own heart. 


\section{Table of Contents}

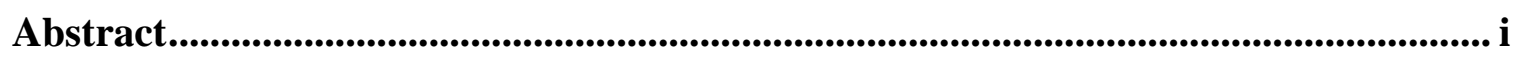

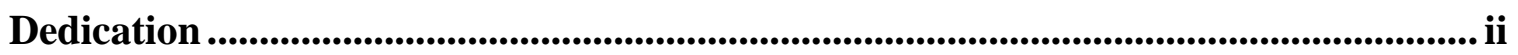

Acknowledgments .................................................................................................................................. iii

List of Tables ........................................................................................................... vi

List of Figures.............................................................................................................................................. vii

List of Schemes ........................................................................................................................... $\mathrm{x}$

1 Glycerol and Propylene Glycol ......................................................................................... 1

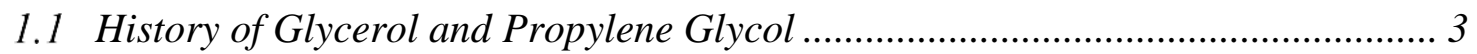

1.1.1 Isolation and Study of Glycerol ................................................................ 3

1.1.2 Isolation and Study of Propylene Glycol ..................................................... 12

1.1.3 Thermal Decomposition of Glycerol and Propylene Glycol ........................ 14

1.2 Glycerol and Propylene Glycol in the Modern Era......................................... 21

1.2.1 Modern Studies of Glycerol and Propylene Glycol Decomposition ............. 22

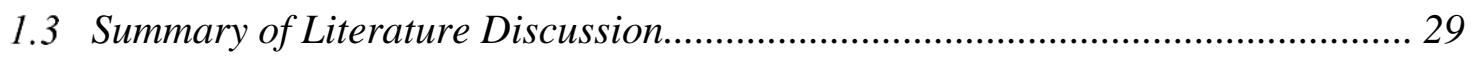

1.4 Nuclear Magnetic Resonance Spectroscopy of Glycerol and Propylene Glycol.. 29

1.4.1 The Utility of NMR Spectroscopy .......................................................... 30

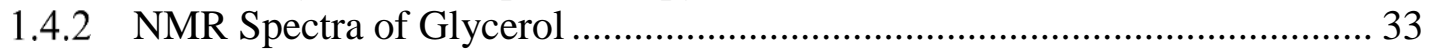

1.4.3 NMR Spectra of Propylene Glycol ............................................................. 38

1.4.4 NMR Spectra of Mixed Propylene Glycol and Glycerol.............................. 45

1.4.5 NMR Spectra of Vaporized Propylene Glycol and Glycerol......................... 46

1.5 Outline of Subsequent Chapters....................................................................... 49

2 Electronic Cigarettes .......................................................................................................... 51

2.1 How Electronic Cigarettes Work .................................................................... 51

2.2 The Rise and Regulation of Electronic Cigarettes........................................... 52

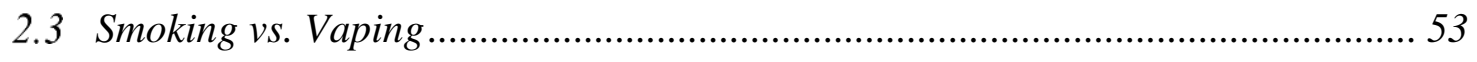

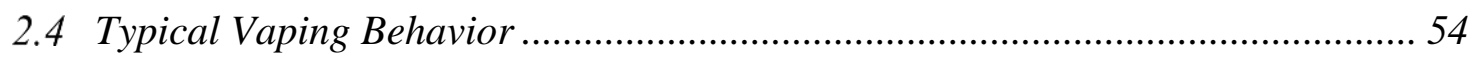

2.5 Electronic Cigarette Device Diversity ……………...................................... 55

2.5.1 The E-Liquid Reservoir and Heating Element.............................................. 55

2.6 Reports of Degradation by Electronic Cigarettes in the Literature ...................... 60

$3 \quad$ Electronic Cigarette Liquids...................................................................................... 62 
3.1 Electronic Cigarette Liquid Composition ........................................................... 62

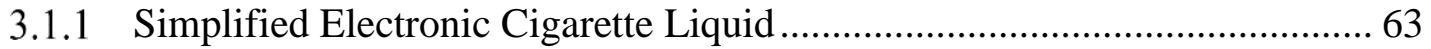

3.2 Collecting Samples of Vaporized Electronic Cigarette Liquid for NMR Analysis 64

3.3 Electronic Cigarette Liquid Consumption ........................................................... 66

3.4 Electronic Cigarette Liquid Degradation .......................................................... 69

3.4.1 Identification and Confirmation of Decomposition Products ........................ 73

3.4.2 Glycerol Degradation........................................................................... 75

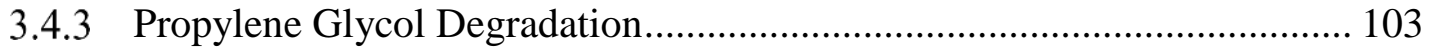

3.5 Quantification of Decomposition Products ...................................................... 118

3.5.1 Quantitative Sample Collection ............................................................ 119

3.5.2 Quantification of Hydroxyacetone and Acetaldehyde ............................... 122

3.5.3 Quantification Summary ……………………………………………….... 130

3.6 Oxygen Dependence of Decomposition …………........................................ 130

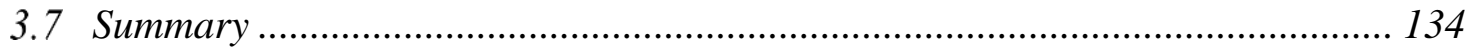

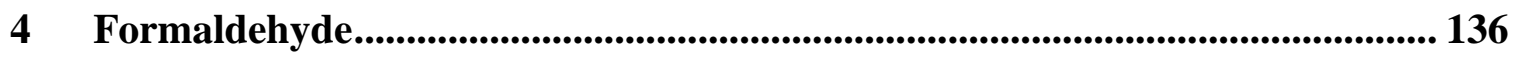

4.1 Practical Characteristics of Formaldehyde .................................................... 136

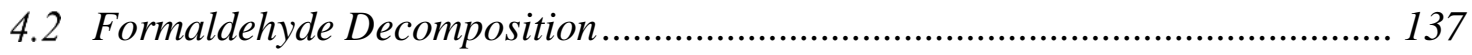

4.3 Initial Discovery of Formaldehyde ………………...................................... 138

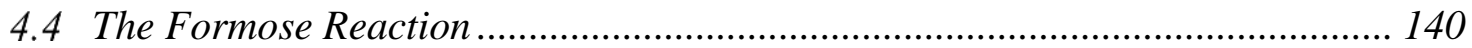

4.5 Formaldehyde as a Degradation Product of Glycerol and Propylene Glycol ... 141

4.5.1 Formaldehyde as a Degradation Product of Electronic Cigarette Liquids . 142

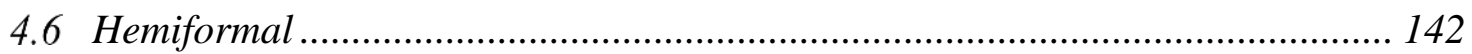

4.6.1 Hemiformal Detection by Nuclear Magnetic Resonance Spectroscopy ..... 146

4.6.2 Hemiformal Solvolysis ............................................................................ 148

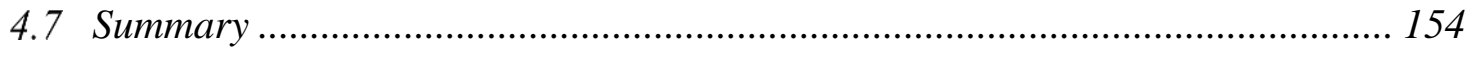

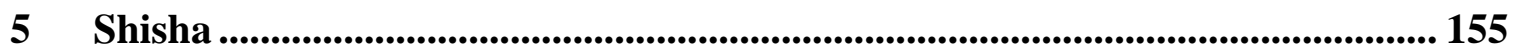

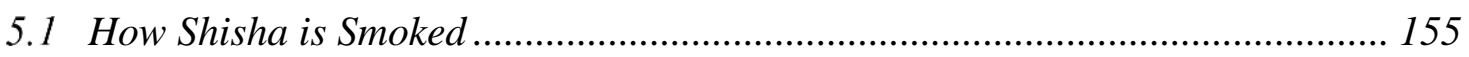

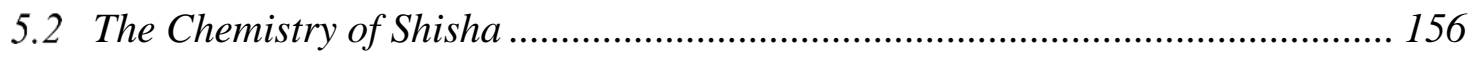

5.3 Smoking, Filtration, and Degradation ……………..................................... 157

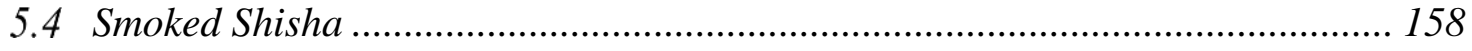

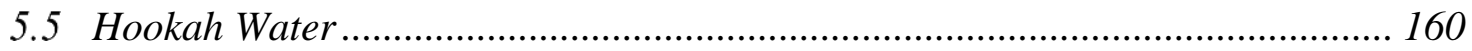

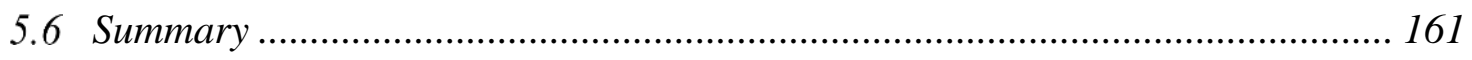

6 Conclusions and Future Work........................................................................................ 163

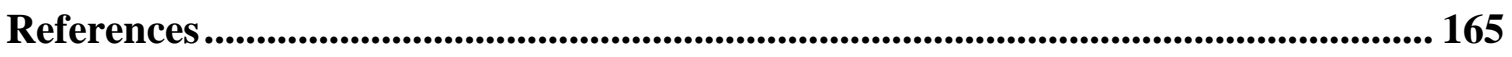




\section{List of Tables}

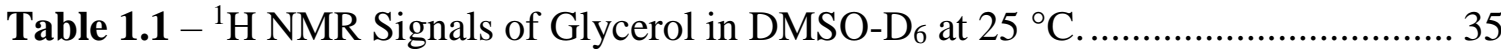

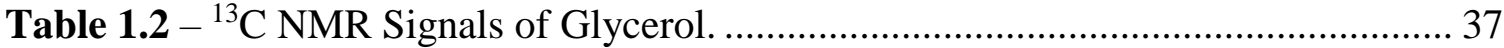

Table 1.3 - ${ }^{17} \mathrm{O}$ NMR Signals of Glycerol............................................................... 38

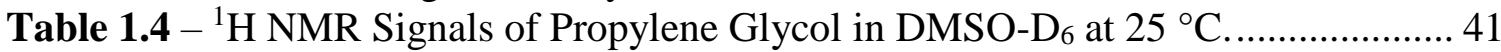

Table 1.5 $-{ }^{13} \mathrm{C}$ NMR Signals of Propylene Glycol. .................................................... 43

Table 1.6 - ${ }^{17} \mathrm{O}$ NMR Signals of Propylene Glycol...................................................... 45

Table 3.1 - Conditions and Consumption of Samples for Quantification of Degradation

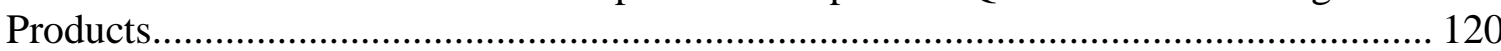

Table 3.2 - Quantification of Hydroxyacetone................................................... 123

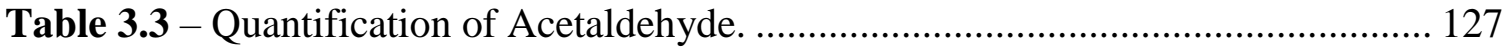

Table 3.4 - Conditions and Consumption of Aerobic/Anaerobic Samples. .................. 131

Table 4.1 - Initial Composition of Methoxymethanol Hydrolysis Samples................. 150

Table 4.2 - Equilibrium Concentrations and $K_{\text {eq }}$ of Methoxymethanol Hydrolysis at 25 ${ }^{\circ} \mathrm{C}$. 


\section{List of Figures}

Figure 1.1 - Molecular Structures of Glycerol and Propylene Glycol. .......................... 1

Figure 1.2 - Non-Equivalent Protons of Glycerol. ....................................................... 33

Figure 1.3 - The ${ }^{1} \mathrm{H}$ NMR Spectrum of Glycerol in DMSO-D 6 at $25{ }^{\circ} \mathrm{C}$....................... 34

Figure 1.4 - Assignment of the ${ }^{1} \mathrm{H}$ Spectrum of Glycerol in DMSO-D 6 at $25{ }^{\circ} \mathrm{C} \ldots \ldots \ldots . . .34$

Figure 1.5 - Non-Equivalent Carbons of Glycerol. .................................................... 36

Figure 1.6 - The ${ }^{13} \mathrm{C}$ NMR Spectrum of Pure Glycerol at $100{ }^{\circ} \mathrm{C}$................................... 36

Figure 1.7 - Assignment of the ${ }^{13} \mathrm{C}$ NMR Spectrum of Pure Glycerol at $100{ }^{\circ} \mathrm{C}$........... 37

Figure 1.8 - Non-Equivalent Oxygens of Glycerol..................................................... 37

Figure 1.9 - The ${ }^{17} \mathrm{O}$ NMR Spectrum of Pure Glycerol at $100{ }^{\circ} \mathrm{C}$................................ 38

Figure 1.10 - Non-Equivalent Protons of Propylene Glycol....................................... 39

Figure 1.11 - The ${ }^{1} \mathrm{H}$ NMR Spectrum of Propylene Glycol in DMSO-D 6 at $25{ }^{\circ} \mathrm{C} \ldots . . .40$

Figure 1.12 - Assignment of the ${ }^{1} \mathrm{H}$ Spectrum of Propylene Glycol in DMSO-D 6 at 25

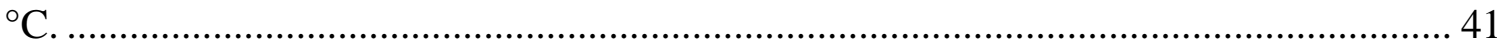

Figure 1.13 - Non-Equivalent Carbons of Propylene Glycol...................................... 42

Figure 1.14 - The ${ }^{13} \mathrm{C}$ NMR Spectrum of Pure Propylene Glycol at $100{ }^{\circ} \mathrm{C}$................. 42

Figure 1.15 - Assignment of the ${ }^{13} \mathrm{C}$ NMR Spectrum of Pure Propylene Glycol at 100

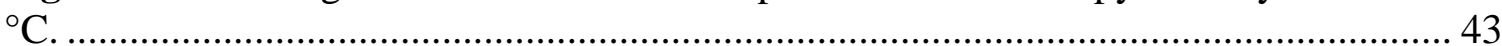

Figure 1.16 - Non-Equivalent Oxygens of Propylene Glycol. ...................................... 44

Figure 1.17 - The ${ }^{17} \mathrm{O}$ NMR Spectrum of Pure Propylene Glycol at $75{ }^{\circ} \mathrm{C}$.................. 44

Figure 1.18 - Incomplete Resolution Between ${ }^{1} \mathrm{H}$ Resonances of PG (red) and GL (blue)

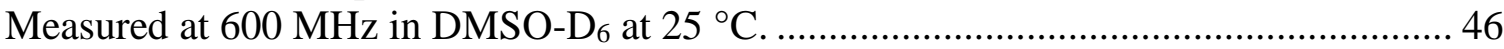

Figure 1.19 - Full ${ }^{1} \mathrm{H}$ NMR Spectrum of Pure (PG/GL) in DMSO-D 6 at $25^{\circ} \mathrm{C} \ldots \ldots \ldots . . . .47$

Figure 1.20 - The ${ }^{1} \mathrm{H}$ NMR Spectrum of Vaporized (PG/GL), Plotted in Blue, Contains

Peaks Not Observed in Unvaporized (PG/GL), Plotted in Grey. ................................... 48

Figure 1.21 - The Aldehyde Region of Vaporized (PG/GL), Plotted in Blue, and Unvaporized (PG/GL), Plotted in Grey.

Figure 2.1 - Comparison of (PG/GL) Decomposition by Cartomizers of Different Design

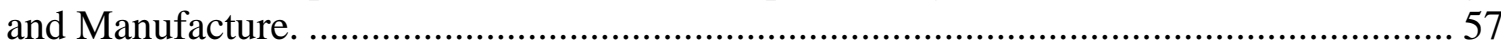

Figure 2.2 - Replacement Coil Comparison................................................................... 59

Figure 3.1 - Vaporized Sample Collection Apparatus. ............................................ 65

Figure 3.2 - Consumption of (PG/GL) Increases with Increased Operating Power........ 67

Figure 3.3 - Consumption of (PG/GL) Increases with Increased Puff Duration............. 68

Figure 3.4 $-{ }^{1} \mathrm{H}$ NMR Spectra of (PG/GL) Vaporized Using Low Power Settings........ 70

Figure 3.5 - Increased Abundance and Diversity of Decomposition Products with Increased Power.

Figure 3.6 - Selected Peaks from the ${ }^{1} \mathrm{H}$ NMR Spectra of (PG/GL) Samples Vaporized with Increasing Power....................................................................................... 72

Figure 3.7 - Confirmation of Decomposition Product Identity by Minute Addition. .... 75

Figure 3.8 - Molecular Structure of Hydroxyacetone. ............................................. 78

Figure 3.9 - ${ }^{1} \mathrm{H}$ NMR Assignment of Hydroxyacetone and Confirmation in Vaporized (PG/GL).

Figure 3.10 - Molecular Structure of Acrolein. 
Figure 3.11 - ${ }^{1} \mathrm{H}$ NMR Assignment of Acrolein and Confirmation in Vaporized (PG/GL).....

Figure 3.12 - Molecular Structure of Glycidol. 82

Figure 3.13 - ${ }^{1} \mathrm{H}$ NMR Assignment of Glycidol and Confirmation in Vaporized (PG/GL).

Figure 3.14 - Molecular Structure of Acetaldehyde.

Figure 3.15 - ${ }^{1} \mathrm{H}$ NMR Assignment of Acetaldehyde and Confirmation in Vaporized (PG/GL).

Figure 3.16 - Molecular Structure of Dihydroxyacetone.

Figure 3.17 - ${ }^{1} \mathrm{H}$ NMR Assignment of Dihydroxyacetone and Confirmation in Vaporized (PG/GL).

Figure 3.18 - Molecular Structure of Glyceraldehyde.

Figure 3.19 - ${ }^{1} \mathrm{H}$ NMR Assignment of Glyceraldehyde and Confirmation in Vaporized (PG/GL).

Figure 3.20 - Molecular Structure of Glycolaldehyde.

Figure 3.21 - ${ }^{1} \mathrm{H}$ NMR Assignment of Glycolaldehyde and Confirmation in Vaporized (PG/GL).

Figure 3.22 - Molecular Structure of Allyl Alcohol

Figure 3.23 - ${ }^{1} \mathrm{H}$ NMR Assignment of Allyl Alcohol and Confirmation in Vaporized (PG/GL) ....

Figure 3.24 - Molecular Structure of Acetic Acid.

Figure 3.25 - ${ }^{1} \mathrm{H}$ NMR Assignment of Acetic Acid and Confirmation in Vaporized (PG/GL).

Figure 3.26 - Molecular Structure of Formic Acid.

Figure 3.27 - ${ }^{1} \mathrm{H}$ NMR Assignment of Formic Acid and Confirmation in Vaporized (PG/GL).

Figure 3.28 - Molecular Structure of Propanal.

Figure 3.29 - ${ }^{1} \mathrm{H}$ NMR Assignment of Propanal and Confirmation in Vaporized

(PG/GL).

Figure 3.30 - Molecular Structures of E,Z-prop-1-ene-1-ol.

Figure 3.31 - Tautomerization of E,Z-prop-1-ene-1-ol to Propanal by ${ }^{1} \mathrm{H}$ NMR

Figure 3.32 - Molecular Structure of Acetone.

Figure 3.33 - ${ }^{1} \mathrm{H}$ NMR Assignment of Acetone and Confirmation in Vaporized (PG/GL).

Figure 3.34 - Molecular Structure of Lactaldehyde.

Figure 3.35 - ${ }^{1} \mathrm{H}$ NMR Assignment of Lactaldehyde and Confirmation in Vaporized (PG/GL).

Figure 3.36 - Increased Consumption of (PG/GL) with Increased Operating Power and

Puff Duration.

Figure 3.37 -Hydroxyacetone Values Averaged from Three Devices. ....................... 124

Figure 3.38 - Hydroxyacetone Values From Each Device, Separated by Puff Duration.

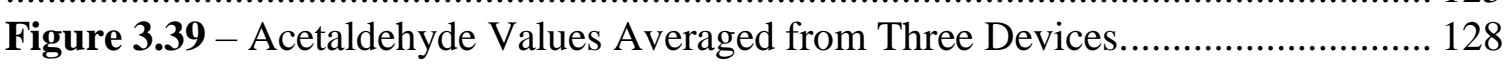

Figure 3.40 - Acetaldehyde Values From Each Device, Separated by Puff Duration. . 129 
Figure 3.41 - Averaged ${ }^{1} \mathrm{H}$ Spectra (PG/GL) Vaporized Under Anaerobic and Aerobic Conditions.

Figure 3.42 - Quantification of Hydroxyacetone and Glycolaldehyde in Anaerobic and Aerobic Samples.

Figure 3.43 - Averaged Values of Hydroxyacetone and Glycolaldehyde in Anaerobic and Aerobic Samples.

Figure 4.1 - Molecular Structure of Formaldehyde................................................... 136

Figure 4.2 - Formaldehyde Bubbling Apparatus.................................................... 145

Figure 4.3 - Hemiformal Assignment and Confirmation by ${ }^{1} \mathrm{H}$ NMR Spectroscopy.... 148

Figure 4.4 - Varied Concentrations of Methoxymethanol in $\mathrm{D}_{2} \mathrm{O}$ by ${ }^{1} \mathrm{H}$ NMR

Spectroscopy at $25{ }^{\circ} \mathrm{C}$.

Figure 4.5 - Comparison of Measured Ratios and Calculated Equilibrium Constants. 152

Figure 4.6 - Van't Hoff Plot of Methoxymethanol Hydrolysis. 153

Figure 5.1 - ${ }^{1} \mathrm{H}$ NMR Spectra of Smoked (red) and Unsmoked (black) Shisha........... 159

Figure 5.2 - ${ }^{1} \mathrm{H}$ NMR Spectra of Smoked Shisha and Water from the Hookah. 161 


\section{List of Schemes}

Scheme 1.1 - Josef Redtenbacher's Isolation of Acrolein from Glycerol. ...................... 10

Scheme 1.2 - John Nef's Pathway of Glycerin Dissociation.......................................... 16

Scheme 1.3 - Hemiacetal Formation and Acetal Condensation of Aldehydes with

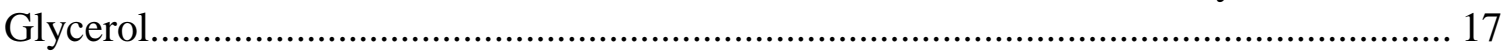

Scheme 1.4 - Sabatier \& Gaudin's Metal-Catalyzed Pathway for the Fragmentation of

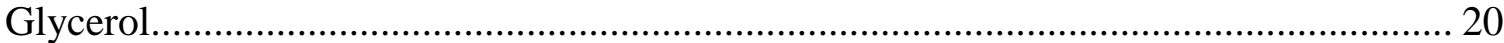

Scheme 1.5 - The Fate of ${ }^{13} \mathrm{C}$ during Glycerol Pyrolysis.......................................... 25

Scheme 1.6 - Oxirane Intermediate Formation in Glycerol and Propylene Glycol

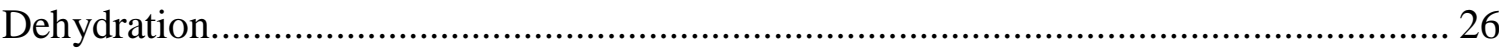

Scheme 1.7 - Propylene Glycol Combustion pathway proposed by Díaz et al. ............. 28

Scheme 3.1 - Double Dehydration of Glycerol into Acrolein Proposed by Nef. ........... 76

Scheme 3.2 - Dehydration and Subsequent Degradation of Glycerol as Discussed by

Nef.

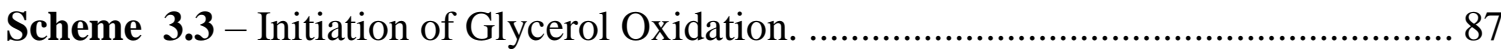

Scheme 3.4 - Glycerol Oxidation - Oxy-alkyl Radicals............................................. 88

Scheme 3.5 - Glycerol Oxidation - Hydroxyl-alkyl Radicals......................................... 89

Scheme 3.6 - Formation of Glycerol $\beta$-Formate........................................................... 101

Scheme 3.7 - Aerobic Thermal Decomposition of Glycerol. ........................................ 102

Scheme 3.8 - Dehydration of Propylene Glycol as Discussed by Nef, Laino et al., and

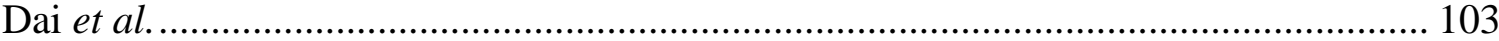

Scheme 3.9 - Initiation of Propylene Glycol Oxidation. ................................................. 110

Scheme 3.10 -Propylene Glycol Oxidation - Oxy-alkyl Radicals. .............................. 111

Scheme 3.11 - Propylene Glycol Oxidation - Alkyl and Hydroxyl-alkyl Radicals..... 113

Scheme 3.12 - Aerobic Thermal Decomposition of Propylene Glycol......................... 117

Scheme 4.1 - Stable Forms of Concentrated Formaldehyde. ....................................... 137

Scheme 4.2 - Thermal Decomposition Routes of Formaldehyde in Aerobic and Anoxic

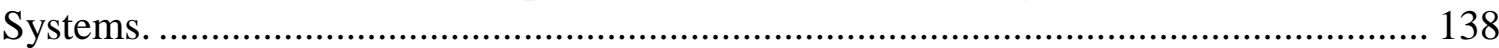

Scheme 4.3 - Butlerov's Failed Synthesis of Methylglycol or Butlerov's Accidental

Synthesis of Paraformaldehyde.................................................................................. 139

Scheme 4.4 - A Simplified Representation of the Initial Steps of the Formose Reaction.

Scheme 4.5 - Hemiacetal Formation by Addition of Formaldehyde to an Alcohol..... 143

Scheme 4.6 - Reversible Formation of Hemiformal Compound Methoxymethanol.... 143

Scheme 4.7 - Glycerol and Propylene Glycol Mono-Hemiformals................................ 146

Scheme 4.8 - Hydrolysis of Methoxymethanol. .......................................................... 149 


\section{Glycerol and Propylene Glycol}

The chemical compounds 1,2,3-propanetriol (glycerol, glycerine, GL) and 1,2-propanediol (propylene glycol, PG) are clear, colorless liquids at room temperature and atmospheric pressure. Propylene glycol and glycerol are relatively small and simple molecules having chemical formulae and structures (shown in Figure 1.1) that have been understood by organic chemists since the $19^{\text {th }}$ century. These propanols are generally recognized as safe for moderate oral consumption and are utilized in a number of food, drug, and personal care applications owing to their favorable physical properties; glycerol in particular is an exceptionally viscous liquid of remarkably low volatility, and even happens to have a sweet taste. When used as solvents these liquids form a highly protic environment thick with hydrogen bonding. Each having multiple hydroxyl groups, glycerol and propylene glycol form strong intermolecular associations, making them viscous as liquids and biasing them towards aerosol droplet formation once vaporized.
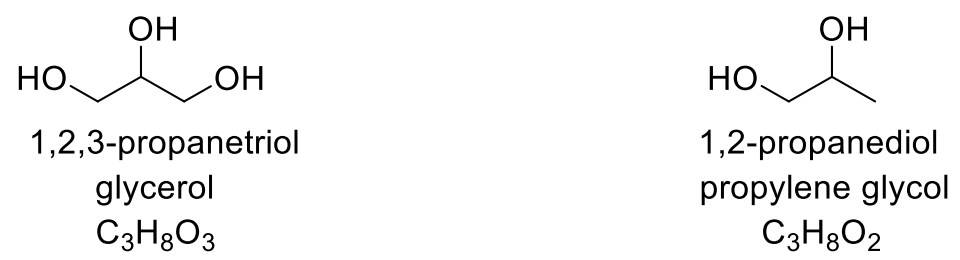

Figure 1.1 - Molecular Structures of Glycerol and Propylene Glycol.

The chemical properties and reactivity of propylene glycol and glycerol are typical of alcohols, although their multiple hydroxyl groups add degrees of complexity to the wellestablished chemistry in which they participate ${ }^{1-3}$ Each is slightly hygroscopic and 
miscible with water and with other alcohols, including one another. Similar to more simple alcohols such as 1-propanol and 2-propanol, at elevated temperatures propylene glycol and glycerol are susceptible to dehydration/pyrolysis and to oxidation/combustion, forming aldehydes and carboxylic acids before decarboxylation or fragmentation via radical bond cleavage. ${ }^{4-7}$ The identity, quantity, and reactivity of products resulting from this thermal decomposition are the central subjects of discussion within this dissertation, which seeks to describe how glycerol and propylene glycol can degrade when they are vaporized using an electronic cigarette.

Vaporization of glycerol and propylene glycol requires relatively high temperatures purification of glycerol by distillation must be performed under vacuum because it begins to polymerize and decompose at temperatures below its boiling point of $290{ }^{\circ} \mathrm{C}$ under atmospheric pressure. Propylene glycol, having one fewer hydroxyl group than glycerol, is somewhat less dense and viscous than the triol, boiling at $189{ }^{\circ} \mathrm{C}$ under atmospheric pressure and distilling without decomposition. What is known about the thermal degradation and the relevant history of these molecules is summarized in this Chapter, using primary sources when available. Please see Chapter 2 for a brief introduction to electronic cigarettes. 


\subsection{History of Glycerol and Propylene Glycol}

\subsubsection{Isolation and Study of Glycerol}

\subsubsection{Initial Discovery by Carl Scheele}

Glycerol is a naturally-occurring compound that is abundant in biomass as a component of glyceride fats, which utilize the molecule as their structural backbone. It was observed by legendary Swedish apothecary and chemist Carl Wilhelm Scheele that treatment of fats with lead calx ${ }^{\mathrm{I}}$ in boiling water resulted in the formation of a sweet water-soluble substance that was thick like syrup. Scheele referred to this compound as Süssigkeit in German and sótma in Swedish, both of which translate roughly to "sweetness" in English. Scheele's work was reported to the Swedish Royal Academy in the year $1783,{ }^{8}$ at a time in the history of chemistry when Antoine Lavoisier's description of oxygen gas was still struggling to overcome the phlogiston theory of combustion. A German communication of the discovery was published in Crell's Chemische Annalen the following year; in this article Scheele provides the date of his original discovery of the "sweetness" by making reference to a doctoral dissertation - published in Latin by the University in Göettingen in 1779 and later in German in Crell's Chemisches Journal in 1780 - in which his discovery was discussed. ${ }^{9-}$ 11

\footnotetext{
I Translated from bly-kalk in Swedish, this is an archaic name for lead(II) oxide (lead monoxide, $\mathrm{Pb}^{\mathrm{II}} \mathrm{O}$ ), which is also known by its mineral name litharge. During the phlogiston era in which Scheele was writing, the calx of metals such as mercury and lead were generated by heating the elemental forms of those metals in air.
} 
Scheele discovered this "sweetness" in his laboratory while conducting a procedure to make emplastrum simplex, a lead soap used by pharmacists of his day as a plaster for the treatment of skin ailments. ${ }^{12,13}$ This plaster was made by combining olive oil and litharge in water and boiling; Scheele conducted this procedure in a fire-heated cauldron, stirring his boiling solution with a spade. After boiling this aqueous mixture of triglyceride fats and lead monoxide, the emplastrum simplex separates from the aqueous phase and forms a solid that is isolated. Scheele observed that after cooling the mixture and removal of the lead soap and residual oil, evaporation of the water from the leftover aqueous phase yielded the sweet compound. He isolated this "sweetness" from a wide variety of plant and animal sources, empirically determining that it was a principle component of many fats. Scheele investigated this sweet material, finding it chemically distinct from sugar and honey in that it would not crystalize or ferment, was soluble in an alkali tincture from which sugars were known to separate, and was more resistant than sugar to heat, though its vapors could be ignited by a candle and the substance would catch fire if placed upon hot coals.

Scheele distilled the sweet compound, determining that the substance gave off water when heated, formed an empyreal but still sweet-tasting vapor when heated further, and formed an acrid brown smoke when heated further still, eventually condensing into a black oil that was bitter and pungent. After this distillation, Scheele noted that some shiny carbon residue remained in his retort, but there was no trace of residual lead. Among the more interesting observations that Scheele made regarding the sweetness was that it was volatile enough to distill, but that such a distillation required nearly the same amount of heat as was required 
to distill vitriolic acid, ${ }^{\mathrm{II}}$ and that this amount of heat was sufficient to destroy the material after repeated distillations, leaving it more acrid and bitter with each successive transfer. ${ }^{14}$

Thus, Carl Scheele was the first chemist to isolate and study glycerol, which he correctly identified as a principle component of fats. During his investigation, Scheele established that the compound was susceptible to pyrolysis and thermal decomposition, and as such could not be purified by simple distillation. From his descriptions of these distillations it is clear that he was the first person to taste and inhale vaporized glycerol; he almost certainly inhaled a mixture of glycerol decomposition products as well. The eminent $20^{\text {th }}$ century author and chemist Isaac Asimov wrote of Scheele that to taste the substances of his investigations was a central aspect of his chemical analyses, and that this routine exposure to toxic metals and acids is widely regarded as a contributing factor to his early death at 43 years old. ${ }^{15}$ It is said that Carl Scheele made more original discoveries during his short life than all the other notable chemists ${ }^{\mathrm{III}}$ of his era combined. ${ }^{16,17}$ In the decades following Scheele's identification of the "sweetness" and the publication of his preparation technique, the compound became known as principe doux de huiles or principe doux de Scheele in the French chemical literature, and Scheeles Süss or Scheeleschen Süss in the German literature. ${ }^{\text {IV }}$

\footnotetext{
${ }^{\text {II }}$ Sulfuric acid; boiling point $337^{\circ} \mathrm{C}$

III This is astonishing, considering that Scheele was contemporary with Joseph Black, Henry Cavendish, Joseph Priestly, and even Lavoisier himself.

IV I can find no support in the original German publications for the legend that Scheele had himself referred to the compound as Oelsüss, although he may have done so in private communications to which I am not privy.
} 


\subsubsection{Further Investigation by Michel Chevreul}

Working in Paris in 1811, French chemist Michel Eugène Chevreul began investigating the chemistry of fats and soaps at the behest of his teacher Louis Nicolas Vauquelin. ${ }^{18}$ Chevreul had come to Paris as a young man seeking to work under Vauquelin, a famous chemist who had been a student of Lavoisier. Chevreul carefully studied the saponification process and reported his observations in a series of communications to Annales de Chimie ${ }^{\mathrm{V}}$ beginning with the publication of the first memoir in 1813 and ending with the seventh (which he split into three parts) in $1817 \cdot{ }^{19-27}$ This series of publications was titled Recherches Chimiques sur Plusieurs Corps Gras et Particulierement sur Leurs Combinaisons Avec les Alkalis, which I translate to read "Chemical Research on Several Fats and Especially on Their Combinations with Alkalis" in English. In a letter to the editors immediately preceding the second memoir of this series, published in 1815 , Chevreul made his first mention of principe doux de huiles (sweet principle of oils), which he found remaining in the aqueous mother liquor after saponification. ${ }^{20,28}$ In an echo of Scheele's findings that glycerol could not simply be distilled to purity, Chevreul noted in this work that the principe doux he had recovered from pork fat - which he had attempted to purify by distillation - had a slight yellow color that he believed was the result of some foreign matter. In seeking to prove that principe doux was colorless when pure, Chevreul carefully prepared the compound by mixing litharge with olive oil under gentle heat, showing that he was familiar with the work of Scheele. In the third memoir of the series,

\footnotetext{
${ }^{\vee}$ By 1816, Annales de Chimie had become Annales de Chimie et de Physique.
} 
published later in the same volume of Annales de Chimie, Chevreul gives proper reference to Scheele's description of glycerol made three decades earlier. ${ }^{21}$

Using only glassware and a balance, at a time several decades before Kekulé and Couper articulated structural theories resembling modern notions of valence, Chevreul discovered that neutral fats absorbed water and dissociated into free fatty acids and principe doux in aqueous solutions when treated with a wide variety of bases or metal-oxides. Chevreul's experiments showed that fats in general were simply combinations of these compounds, and he brilliantly associated different chemical combinations of fatty acids and principe doux with the different physical properties of solid fats and liquid oils, establishing the foundation of our modern understanding of glyceride chemistry. ${ }^{29}$ Chevreul surveyed an even wider variety of fat sources than Scheele, studying the chemistry of glycerides so thoroughly that he correctly determined that sperm whale oil did not contain principe doux. He began referring to principe doux as glycérine ${ }^{\mathrm{VI}}$ in a celebrated monograph titled Recherches Chimiques sur les Corps Gras d'Origine Animale published in Paris in 1823, translating to "Chemical Research on Fats of Animal Origin" in English. ${ }^{30}$ The name glycérine has evolved to include glycerin as an accepted spelling typically encountered in English and German literature and glycerol as the modern preferred chemical nomenclature. Like Carl Scheele, Chevreul reported the smell and taste of each compound he studied. Unlike Scheele, who died young and in relative obscurity, Chevreul would go

\footnotetext{
${ }^{\mathrm{VI}}$ Despite numerous uncited reports that Chevreul had originally used the name glycérine - from the translation of the French "doux" to the Greek " $\gamma \lambda v \kappa o ́ \varsigma$ " (glykós) - as early as 1811, I can find no support for such claims in the primary sources; Chevreul was still referring to glycerol as principe doux in the seventh memoire of his series, which was published in 1817.
} 
on to become one of the most respected academics and beloved members of $19^{\text {th }}$ century French society, dying in 1889 at 103 years old. Following the publication of Chevreul's observations other chemists in Europe began preparing and experimenting with glycerol, including such illustrious figures as Joseph Louis Gay-Lussac, Jean-Baptiste Dumas, Justus von Liebig, and Théophile-Jules Pelouze.

\subsubsection{Further Investigation by Jules Pelouze}

In 1836, Pelouze published a Memoire sur la Glycérine in Annales de Chimie et de Physique, in which he discussed the early history of the molecule, crediting his compatriot Chevreul along with their predecessor Scheele for all that was known about glycerol at that time. ${ }^{31}$ In his own investigations, Pelouze found that entirely removing water from glycerol was difficult, but by lightly heating the material under vacuum for many hours he achieved sufficient purity to establish the nearly correct empirical formula of $\mathrm{C}_{6} \mathrm{H}_{16} \mathrm{O}_{6}$ - wrong by only a factor of two. ${ }^{\text {VII }}$ Pelouze wrote that glycerol divided into two parts when heated, one of which evaporated without alteration, while the other decomposed into empyreumatic oils, acetic acid, flammable gases and a carbonaceous residue. Carl Scheele's publications give a remarkably similar account and seem to have influenced these observations. Pelouze subjected glycerol to a number of acids and metal oxides, finding in particular that treatment with nitric acid resulted in decarboxylation, leading to the formation of carbonic acid and oxalic acid, echoing Scheele's findings from 1783 that treatment of his sweet

\footnotetext{
VII Pelouze made this determination based on the equivalent weight instead of atomic weight, accidently doubling his empirical formula in an error common to his time. This discrepancy would be rectified after the Karlsruhe Congress of 1860 helped establish the modern understanding of atomic weights.
} 
compound with nitric acid resulted in "sugar acid" - his name for oxalic acid. Pelouze described glycerol as being d'une couleur très légèrement jaunàtre, which I translate to read "of a very slight yellowish color," reflecting the difficulty of glycerol purification. In the author's opinion, the most important aspect of Pelouze's impact on the understanding of glycerol is that he was the first chemist to study the molecule who was gifted as a writer and able provide a succinct and articulate account of its chemistry, synthesizing the important ideas of Scheele and Chevreul without the linguistic and conceptual burdens of Scheele's era and with more focus and a more sophisticated chemical understanding than Chevreul, whose main concern had been fats and soaps. Like his contemporary Liebig, one might argue that Pelouze made his major impact through his writings and the work of his many his students, although the original discoveries of both men are noteworthy.

\subsubsection{Identification of Acrolein as a Thermal Decomposition Product of Glycerol}

Also published in 1836 was a work by Henri Hess titled Ueber einige Producte der trockenen Destillation, translating roughly to "On some products of dry distillation" in English, which appeared in Annalen der Pharmacie. ${ }^{32}$ In this article Hess described a distillation of purified hemp oil, during which procedure a gas evolved that violently irritated his eyes to tears. Hess described the product as Lampensäure (lamp acid) and said that it possessed properties of the substance described by Liebig under the name Aldehydharz (aldehyde resin). ${ }^{33,34}$ Hess's own mentor, Swedish chemist Jöns Jakob Berzelius, would later discuss this aldehyde compound in a 1839 edition of his celebrated textbook series Lehrbuch der Chemie, ${ }^{35}$ within a short chapter titled Destillationsproducte von fetten Oelen. In this chapter Berzelius credited German chemist Simon Rudolph 
Brandes for having named this substance Acrolein - from the Latin acris and oleum meaning "sharp" and "oil" in English - which Brandes had sought in vain to isolate by distilling fats.

Austrian chemist Josef Redtenbacher, who was among the many student of Liebig, was familiar with the works of Chevreul, Pelouze, Hess, and Berzelius. While working in Liebig's laboratory in the early 1840s Redtenbacher observed that distillation of fatty acids isolated from fats by Chevreul's saponification process did not result in the evolution of acrolein, which he did observe when he distilled the un-saponified fats. Knowing as Chevreul had demonstrated that fats were merely fatty acids joined with glycerol, Redtenbacher added glycerol to a sample of fatty acids and distilled the mixture; he observed the production of acrolein and determined it must therefore be a product of glycerol decomposition. Like his predecessor Brandes, Redtenbacher could not isolate pure acrolein by distilling unprocessed fats, but by distilling refined glycerol in the presence of dehydrating agents ${ }^{\mathrm{VIII}}$ and repeatedly fractionating the volatile products he was eventually able to isolate a compound that distilled at $52{ }^{\circ} \mathrm{C}$ and would likely be considered pure today. This reaction is summarized in Scheme 1.1.

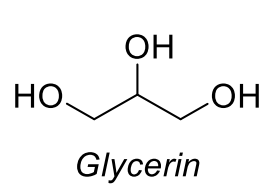

Glycerin

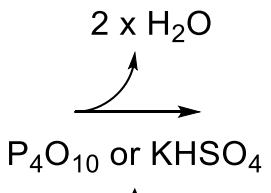

$\Delta$

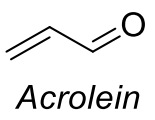

Acrolein

Scheme 1.1 - Josef Redtenbacher's Isolation of Acrolein from Glycerol.

\footnotetext{
VIII Such as wasserfreier Phosphorsaure $\left(\mathrm{P}_{4} \mathrm{O}_{10}\right.$, "anhydrous phosphoric acid" or phosphorus pentoxide) or sauren schwefelsaurem Kali ( $\mathrm{KHSO}_{4}$, "acid sulphate of potash" or potassium bisulfate).
} 
Reporting his findings in an 1843 article to Annalen der Chemie und Pharmacie titled Ueber die Zerlegungsprodukte des Glyceryl-oxydes durch trockene Destillation, Redtenbacher also reported the discovery of the "acetic acid analogue" of acrolein, which he named acrylic acid. ${ }^{36}$

\subsubsection{Additional Discussion of the History of Glycerol}

Glycerol was one of the molecules on which the Golden Era chemists practiced their art. The modern conceptions of alcohol, aldehyde, acetal, carboxylic acid, ether, and ester were developed and articulated while triglycerides, glycerol, and its degradation products were under their earliest investigations. The evolution of the language used to discuss the molecule over its two century history tells this story rather plainly. Carl Scheele and Michel Chevreul had no better way to describe the compound than by taste, though Chevreul recognized that it possessed chemical properties similar to alcohol as well as sugar. Esterification was born when Marcellin Berthelot reversed the saponification process Chevreul had developed and produced synthetic glycerides by combining glycerol with carboxylic acids. ${ }^{37}$ The very name and concept aldehyde (formed from alcoholdehydrogenation) was originally articulated by Liebig less than a decade before acrolein was confirmed to result from glycerol dehydration by his student Redtenbacher. ${ }^{33,34,36}$

While studying in Paris under Pelouze, Italian chemist Ascanio Sobrero learned how to use mixtures of concentrated nitric and sulfuric acids to create condensation conditions favorable for the nitration of alcohols - a technique that he would utilize in 1846 to create nitroglycerine, which he reported gave him a headache upon ingestion. ${ }^{38}$ During the 1850 s, 
when theories about the structure and bonding order of organic molecules were still developing, several major figures in the history of chemistry published accounts of their investigations into the structure and reactivity of glycerol. French chemist Charles Adolphe Wurtz wrote several papers on glycerol and published a synthesis from a halogenated propyl precursor, while the observations of British chemist Alexander Williamson on the synthesis of nitroglycerine helped establish that glycerol was a propane triol - an alcohol with three hydroxyl groups, having one oxygen connected to each carbon of a three carbon chain. ${ }^{39-41}$ Sobrero's acquaintance Alfred Bernhard Nobel, who had also studied under Pelouze, stabilized nitroglycerine using diatomaceous earth in 1862 , and by 1868 had filed patents in the US and Britain for the explosive product he called dynamite, from the Greek

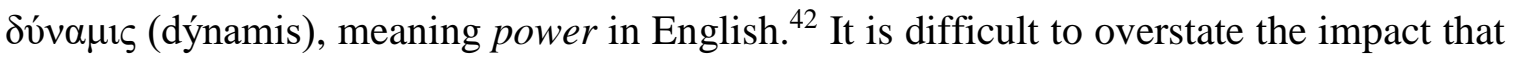
Nobel's explosives had on the industrialization of the United States and Europe, as they allowed humans to change the landscape with unparalleled speed and efficiency. It is interesting to note that both Nobel and Sobrero are documented as having displayed some remorse for the unforeseen effects of their historic inventions.

\subsubsection{Isolation and Study of Propylene Glycol}

The history of propylene glycol is neither as long nor as interesting as the discovery and development of glycerol. Briefly, propylene glycol is a synthetic molecule that is not known to occur naturally and was first prepared by Charles Wurtz. ${ }^{43}$ Writing in 1859 to Annales de Chimie et de Physique, Wurtz mused about the poly-alcoholic nature of glycerol before stating that he had successfully synthesized a series of compounds that he 
considered to be intermediate between ordinary alcohols and glycerol; he referred to these compounds as glycols, and alternatively as alcools diatomiques.

\subsubsection{Initial Synthesis by Charles Wurtz}

Among the four glycols that Wurtz had prepared for the publication - titled Mèmorie sur les Glycols ou Alcools Diatomique - was a compound he called le propylglycol $\left(\mathrm{C}_{3} \mathrm{H}_{8} \mathrm{O}_{2}\right)$, for which the following synthesis was described: alcool amylique (amyl alcohol) is decomposed at high temperature, and the resulting vapors, consisting partially of impure propylene gas, are directed into a vessel and heated with bromine to form what Wurtz called bromure de propylene (1,2-dibromopropane), which can be isolated by fractional distillation; this propylene bromide is heated with silver acetate in glacial acetic acid, yielding impure propylglycol diacetique (diacetyl propylene glycol) which can also be purified by distillation; pure propylene glycol can be obtained by heating this diacetyl product with concentrated baryte caustique (barium hydroxide) or other aqueous bases. Beginning the second stage of this process with 310 grams of propylene dibromide and 540 grams of silver acetate yielded only 45 grams of pure propylene glycol, which boiled at 188-189 ${ }^{\circ} \mathrm{C}$ and did not leave behind any residue when distilled.

Wurtz described pure propylglycol as a colorless, odorless, viscous liquid with a sweet flavor. He subjected this material to a variety of chemical investigations, demonstrating among other things the slow oxidation of propylene glycol into lactic acid in the presence of air and platinum, which was accelerated by addition of various oxidizing agents including nitric acid and potassium permanganate. In contrast with his compatriot 
Chevreul, who had been working some forty years earlier, Wurtz had a nuanced understanding of the structures and reactivity of the molecules he was synthesizing and studying. Production of propylene glycol on a commercial scale began in the United States in 1931, before which time chemists who wished to study or use the compound would generally need to prepare it themselves. ${ }^{2}$

\subsubsection{Thermal Decomposition of Glycerol and Propylene Glycol}

The thermal decomposition of propylene glycol and glycerol was studied by American organic chemist John Ulric Nef, who published his work in a 1904 volume of Liebig's Annalen der Chemie in an article titled Dissociationsvorgänge in der GlycolGlycerinreihe, which I translate to read "Dissociation Reactions in the Glycol-Glycerin Series" in English. ${ }^{44,45}$ In this investigation, which he conducted at the University of Chicago, Nef heated propylene glycol and glycerol by passing them slowly through a pumice-filled combustion tube, collecting the gases that evolved and fractionating the liquid material that exited the tube.

\subsubsection{Decomposition of Glycerol}

In the case of glycerol, Nef heated samples of up to 250 grams to $450{ }^{\circ} \mathrm{C}$, collecting only small volumes of evolved gas, noting a substantial graphite residue left in the tube, and collecting a brown liquid distillate weighing at least $90 \%$ of the initial mass of starting material. This description corresponds very strongly to the results of Carl Scheele's distillation experiments conducted 120 years before, as well as the description provided by Pelouze some 60 years after. Among the evolved gases, Nef identified only carbon 
monoxide and hydrogen. The brown distillate was fractionated, and within the volatile fractions Nef discovered hydroxyacetone, acetaldehyde, formaldehyde, acrolein, and a series of glycerin-acetals that had formed by condensation between these aldehydes and residual glycerol. Nef stated that it took him over a month of work to establish the decomposition products of glycerol, in part due to this recombination of the evolved aldehydes into acetals, and in part because he discovered that hydroxyacetone - which he called acetol - further decomposed into acetaldehyde and formaldehyde at the high temperatures of his experiments. Of his fractionated residue, he stated explicitly that all fractions smelled strongly of formaldehyde after initial collection, and that a residue of insoluble $m$-formaldehyd (1,3,5-trioxane) was left behind on his glassware. Nef contended in his writing that his determinations were made very precisely, offering for our consideration the fact that he had utilized some 1216 grams of glycerol during his dissociation experiments.

John Nef had a very sophisticated understanding of these molecules and the mechanisms of their decomposition - he described in detail the path by which glycerol dehydrates into what he called isomeric dioxypropylidenes. Nef clearly understood, and provided support for his assertion, that these unstable enols would form the stable epoxide Glycid (glycidol) at low temperatures, but at the elevated temperature of the combustion tube instead underwent tautomerization into hydracrylaldehyde (3-hydoxypropanal) and acetol (hydroxyacetone). Each of these products degraded further under the conditions of Nef's experiment; 3-hydroxypropanal is the simplest $\beta$-hydroxyaldehyde (an aldol) and forms 
acrolein nearly quantitatively in a dehydration, while hydroxyacetone partially dissociates into formaldehyde and acetaldehyde. This pathway, summarized in Scheme 1.2, is remarkably similar to those offered by modern theoretical and empirical mechanistic studies.

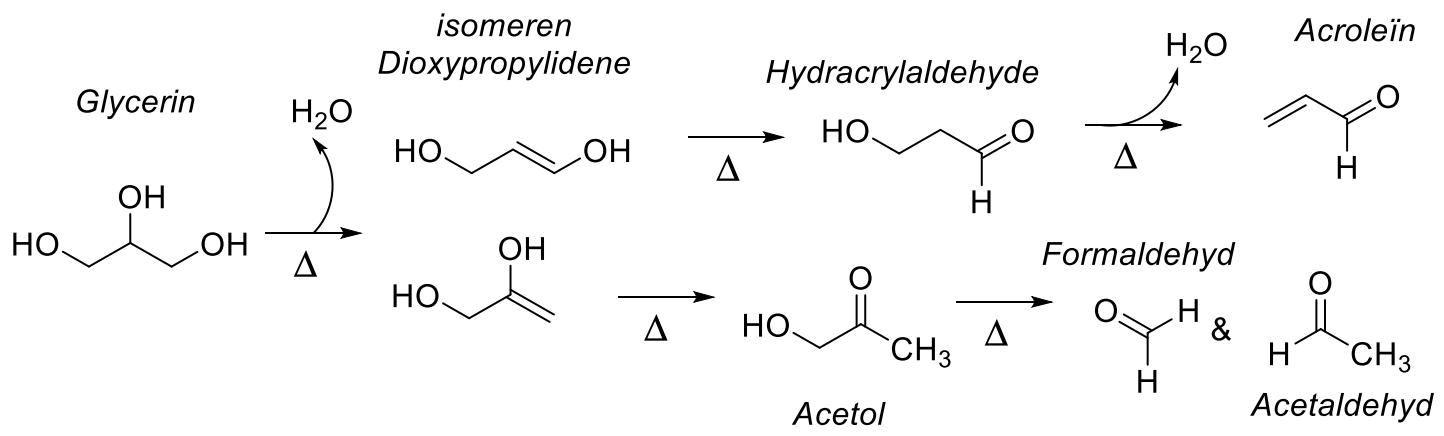

Scheme 1.2 - John Nef's Pathway of Glycerin Dissociation.

\subsubsection{Formation of Acetals and Hemiacetals with Glycerol}

Nef made particular note of Glycerinäthers - cyclic acetals that form between aldehyde decomposition products and residual glycerol - that were present in his volatile fractions. These adducts form between aldehydes and poly-alcohols first by rearrangement into a hemiacetal with one hydroxyl moiety, then by intramolecular condensation with another hydroxyl moiety, as shown in Scheme 1.3. This process becomes increasingly favorable if one of the reactants, such as unreacted glycerol, is present in excess. Having only one hydroxyl moiety left to form intermolecular hydrogen bonds, these acetals are considerably more volatile than glycerol despite the increase in molecular weight, and were thus present among the volatile fractions of Nef's distillation. 


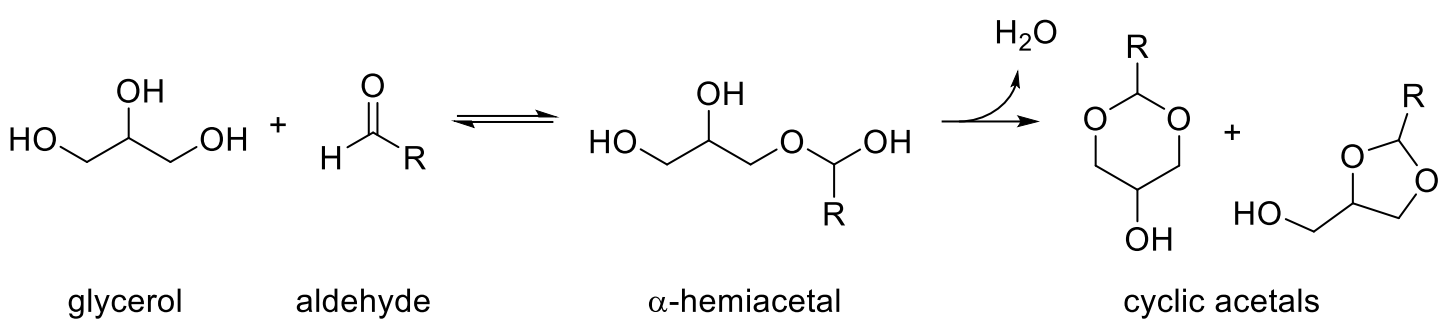

Scheme 1.3 - Hemiacetal Formation and Acetal Condensation of Aldehydes with Glycerol.

Acetals that Nef identified included glycerol adducts with formaldehyde, acetaldehyde, and acrolein. To confirm that these substances had been properly identified, Nef synthesized each in order to study their properties. The acetals of formaldehyde and acetaldehyde Glycerinmonoformal and Acetaldehydglycerin as he called them - had been previously described in the literature, and Nef cites these reports as the source of his synthetic route. ${ }^{46,47}$ The cyclic acetal of formaldehyde combined with propylene glycol, propylglycolformal, had also been described. ${ }^{48}$ Nef made no reference to any description or synthesis of the cyclic acetal between acrolein and glycerol predating his own work he prepared this compound simply by combining glycerol with acrolein in a vessel and heating over boiling water.

\subsubsection{Decomposition of Propylene Glycol}

Propylene glycol was a relatively rare chemical in 1904, so before Nef could study its decomposition, he first had to synthesize the material himself by hydration of propylene oxide. After purification by vacuum distillation, Nef recovered 26 grams of pure propylene glycol, which he heated in his pumice-filled combustion tube at $500{ }^{\circ} \mathrm{C}$, yielding several liters of unidentified gas and $20.8 \mathrm{~mL}$ of liquid distillate representing approximately $80 \%$ 
of the initial volume of material. After fractionating this liquid Nef recovered $11.5 \mathrm{~mL}$ of material which he determined was propylaldehyd (propanal). Nef stated explicitly that he did not find any acetone in this volatile fraction, and suggested that this may indicate that the mechanism of dehydration did not proceed through propylene oxide as an intermediate, reasoning that rearrangement of this epoxide should result in a mixture of both the ketone and the aldehyde products. He also noted that heating propylene glycol to $500{ }^{\circ} \mathrm{C}$ did not result in the same carbonization that he, Scheele, and Pelouze had all observed after heating glycerol.

\subsubsection{Impact of Nef's Investigations}

John Nef's dissociation studies are the foundation of the modern understanding of the thermal decomposition of glycerol and propylene glycol. Most discussions of the subject appearing after their 1904 publication cite them heavily; the relevant sections covering thermal decomposition in the American Chemical Society's Monographs titled Glycerol $\underline{\text { and the Glycols }}$ and The Pyrolysis of Carbon Compounds ${ }^{\mathrm{IX}}$ draw from Nef's work exclusively. ${ }^{3,6}$ The similarity of pumice to quartz makes the heating surface of Nef's apparatus somewhat analogous to modern pyrolysis techniques, but it is not obvious from my reading of Nef's publication what efforts he made to control for the presence of oxygen in his experiments. Although Nef discussed the compounds he investigated as though they were pyrolysis products, acetaldehyde and formaldehyde could also be products of

\footnotetext{
IX In the later ACS Monograph dedicated entirely to Glycerol (Miner \& Dalton, Vol. 117, 1953), which is widely-cited in late $20^{\text {th }}$ century discussions, the subsection on Dehydration and Pyrolysis of Glycerol within the chapter on the Chemical Properties and Derivatives of Glycerol includes a discussion of Nef's work but references Hurd's 1929 ACS monograph as the information source.
} 
numerous decomposition routes involving recombination with free oxygen (i.e. partial combustion reactions) which might occur under an aerobic atmosphere.

\subsubsection{Glycerol Decomposition by Metals}

Not long after the publication of John Nef's results, Nobel laureate Paul Sabatier studied the thermal degradation of glycerol when heated by metals in the presence of air. Sabatier published an article in 1918 in Comptes Rendus along with co-author Georges Gaudin entitled Sur le dédoublement de la glycérine en presence de divers catalyseurs: formation des alcools éthylique et allylique, in which they discuss the effects of a variety of agents known to catalytically affect the thermal decomposition of glycerol. ${ }^{49}$ These authors stated that acrolein was known to evolve during simple distillation of glycerol at atmospheric pressure, and that the addition of certain desiccating agents including potassium bisulfate and aluminum sulfate not only lowered the temperature of this dehydration significantly but also led to the production of other decomposition products. The presence of zinc metal or calcium chloride was known to catalyze the formation of allyl alcohol and hydrogen gas, and the authors discussed similar results from their own investigations using alumina and uranium oxide; however, their more interesting experiments utilized bare metals rather than oxides.

In experiments that are unfortunately not very thoroughly described, Sabatier and Gaudin heated glycerol with nickel ${ }^{\mathrm{X}}$ at $295-310{ }^{\circ} \mathrm{C}$ and with finely-divided copper at $330{ }^{\circ} \mathrm{C}$, both

\footnotetext{
$\mathrm{X}$ No physical description of the metal is given.
} 
metals having been described as freshly reduced into their elemental state. The authors explicitly noted that these temperatures are very near to the boiling point of glycerol. Their experiments yielded acrolein and water as one might expect, but both metals also drove the rapid fragmentation of glycerol, generating vapors that split into condensed liquids and mixed gases which included methane, hydrogen, carbon monoxide, and carbon dioxide. The authors describe the major decomposition route as beginning with a dehydrogenation of glycerol to glyceraldehyde, which itself undergoes fragmentation into carbon dioxide and ethanol in a process which Sabatier analogized to beer yeast fermentation. The resulting ethanol further reacts to produce acetaldehyde by dehydrogenation, then acetaldehyde is divided into carbon monoxide and methane, especially at increased temperatures. This degradation pathway is summarized in Scheme 1.4. Other degradation products that were identified and reported from these experiments included allyl alcohol, propanal, propanol, and ethylene.

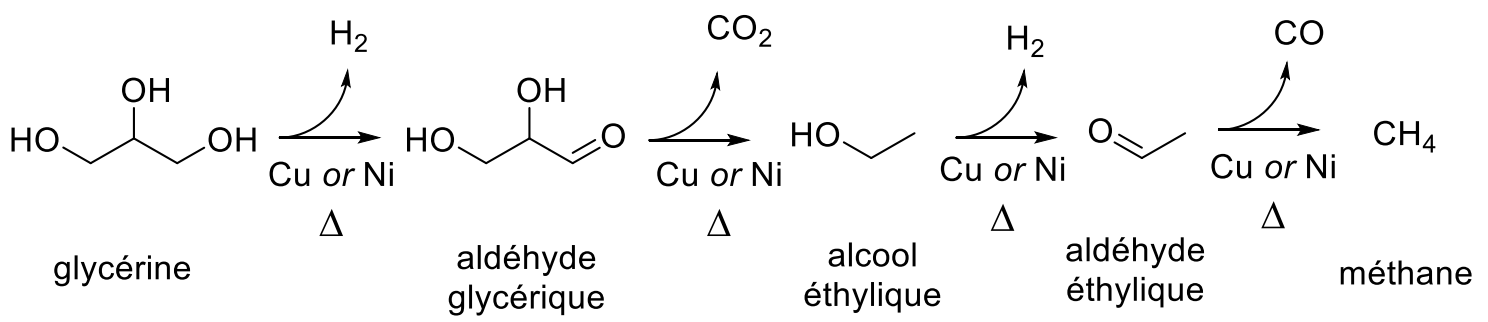

Scheme 1.4 - Sabatier \& Gaudin's Metal-Catalyzed Pathway for the Fragmentation of Glycerol. 


\subsection{Glycerol and Propylene Glycol in the Modern Era}

Millions of tons of glycerol and propylene glycol are now produced annually, generating billions of dollars in revenue and attracting investment and trade in commodities markets. Glycerol remains the subject of much investigation due to its abundance in biomass, making it attractive as a plant-based source of fuel or feedstock for synthetic precursors. ${ }^{50-}$ ${ }^{56}$ The formation of fatty acid methyl esters from triglycerides for use as biofuels generates a large amount of glycerol as a waste product. ${ }^{57,58}$ Oxidation of glycerol can be achieved using a wide variety of oxidants and is catalyzed by a number of metals and compounds but is generally difficult to control, often yielding complex mixtures. ${ }^{59-64}$ Glycerol has long been known to oxidize into eleven possible carbonyl compounds containing the original propyl backbone. ${ }^{1}$ Full combustion of glycerol with free oxygen in air (forming $\mathrm{CO}_{2}$ and $\mathrm{H}_{2} \mathrm{O}$ ) is possible if the material is sprayed into a high temperature environment - complete combustion requires less oxygen than petroleum products. ${ }^{57}$ However, glycerol has a relatively high autoignition temperature and, as Carl Scheele observed, it will not continue burning once heat is removed. The autoignition temperature of glycerol is the subject of some disagreement, with typically cited values ranging from 370 to $523{ }^{\circ} \mathrm{C}$ depending on the contact surface. ${ }^{65,66}$ When considering glycerol as combustible fuel, one might compare the above values with the autoignition of gasoline at $280{ }^{\circ} \mathrm{C}$ and kerosene at $210{ }^{\circ} \mathrm{C}$, both of which will sustain a flame once ignited. ${ }^{57}$ The autoignition temperature of propylene glycol has been reported as $421^{\circ} \mathrm{C}$, citing unpublished data from the U.S. Bureau of Mines with no description of contact surface. ${ }^{66}$ 


\subsubsection{Modern Studies of Glycerol and Propylene Glycol Decomposition}

\subsubsection{Pyrolysis (Anoxic, Anaerobic) vs. Combustion (Oxygenated, Aerobic)}

Modern investigations of the thermal decomposition of propylene glycol and glycerol are almost entirely pyrolysis studies conducted in an anoxic environment, typically heating pure samples of the liquids on a quartz surface under $100 \%$ helium or a similarly inert atmosphere. Writing in the Journal of Physical Chemistry A in 2012, authors Laino et al. stated on the subject of quantitative propylene glycol combustion studies in the literature:

Despite several types of investigations published in the literature, it is remarkable that there has been no attempt to characterize the decomposition of pure $P G$...in the gas phase. This is of paramount importance, because it offers a deep understanding on how easily $P G$...can decompose at high temperature and on the types of chemical products potentially forming during the degradation process. ${ }^{67}$

Expressing a similar sentiment on the subject of glycerol combustion, Barker-Hemings et al. wrote in Combustion Science and Technology in 2012:

To our knowledge, data on the combustion of glycerol in relatively simple systems are not available in the literature to date. ${ }^{68}$

Unfortunately, I must concur with these authors that no complete understanding of the combustion of glycerol or propylene glycol can be drawn from the chemical literature. Despite the long history of investigation into these molecules, their thermal decomposition in air is too complex and depends too heavily on the heating surface and other specific aspects of the system to have been described exhaustively. This problem is complicated by 
the fact that sources sometimes refer to thermal decomposition studies as "pyrolysis" even when they are conducted in the presence of air or controlled percentages of oxygen.

\subsubsection{Modern Pyrolysis Studies of Glycerol and Propylene Glycol}

In 1983, two hundred years after Scheele announced his discovery of glycerol to the Swedish Royal Society, Stein and Antal published a Study of the Gas-Phase Pyrolysis of Glycerol in the Journal of Analytical and Applied Pyrolysis, finding that acrolein, acetaldehyde, carbon monoxide, hydrogen, ethylene, methane, and ethane were present to degrees that varied with the temperature of their experiments, which were conducted at $650-700{ }^{\circ} \mathrm{C}$, heating with steam under an argon atmosphere.$^{69}$ These authors reported that the gas chromatography and infrared spectroscopy methods they used gave no evidence of degradation in samples of glycerol heated with oxygen present to only $200{ }^{\circ} \mathrm{C}$, but that they observed discoloration after heating samples to $250{ }^{\circ} \mathrm{C}$ in air. This work is widely cited in modern discussions of the pyrolysis and thermal degradation of glycerol. The authors stated that they were unable to quantify formaldehyde due to the small fraction present; at the temperatures of their experiments, but they reported its qualitative detection by TLC after derivatization, and they provided a mechanism for its formation. Stein and Antal provided pathways and mechanisms for the formation of acrolein and acetaldehyde, and for their secondary decomposition into the observed gases. The high temperatures used in these experiments produced a different profile of decomposition products than Nef's experiments, which were conducted at $450{ }^{\circ} \mathrm{C}$ and resulted in large amounts of formaldehyde and hydroxyacetone, which appear to have been further degraded. 
The tobacco industry has had a particular interest in the pyrolysis of glycerol and propylene glycol, having long utilized these compounds as humectants in packaged tobacco products. Published internal documents from Philip Morris discuss the addition of glycerol and propylene glycol to cigarettes in the context of increased production of formaldehyde, acetaldehyde, acrolein, glycolaldehyde, propylene oxide, acetone, and propanal..$^{70-72}$ While these documents seek to diminish the significance of any increased production or delivery levels of these decomposition products, it is extremely important to note that the authors of "The Toxic Effects of Cigarette Additives. Philip Morris' Project Mix Reconsidered: An Analysis of Documents Released through Litigation" published in 2011 in PLoS Medicine conclude their investigation by warning that the tobacco industry investigations into cigarette additives cannot be taken simply at face value. ${ }^{73}$

Writing in 2007, researchers from Philip Morris USA in Virginia published an article titled "Carbohydrate Pyrolysis Mechanisms from Isotopic Labeling Part 1: The Pyrolysis of Glycerin: Discovery of Competing Fragmentation Mechanisms Affording Acetaldehyde and Formaldehyde and the Implications for Carbohydrate Pyrolysis" in the Journal of Analytical and Applied Pyrolysis. ${ }^{74}$ In this work, authors Paine et al. focus on the pyrolysis of glycerol as a model compound for understanding the mechanisms of fragmentation of larger and more complex molecules such as carbohydrates. Using isotopically-labeled glycerol isomers, these authors tracked the fate of ${ }^{13} \mathrm{C}$ labels on either the middle or the terminal carbons of glycerol as it decomposed into formaldehyde and acetaldehyde using either a prototype Philip Morris electronic cigarette product or a commercially available 
pyrolysis autosampler; sample analysis was performed by GC/MS. The results of these experiments, summarized in Scheme 1.5, indicated that formaldehyde originates from the terminal carbons, while acetaldehyde results in a binary mixture of labeled species from both products; these findings lead the authors to speculate that under their experimental conditions glycerol undergoes two competing mechanisms of unimolecular fragmentation, although they also provide a third, non-synchronous mechanism involving unstable enol intermediates.

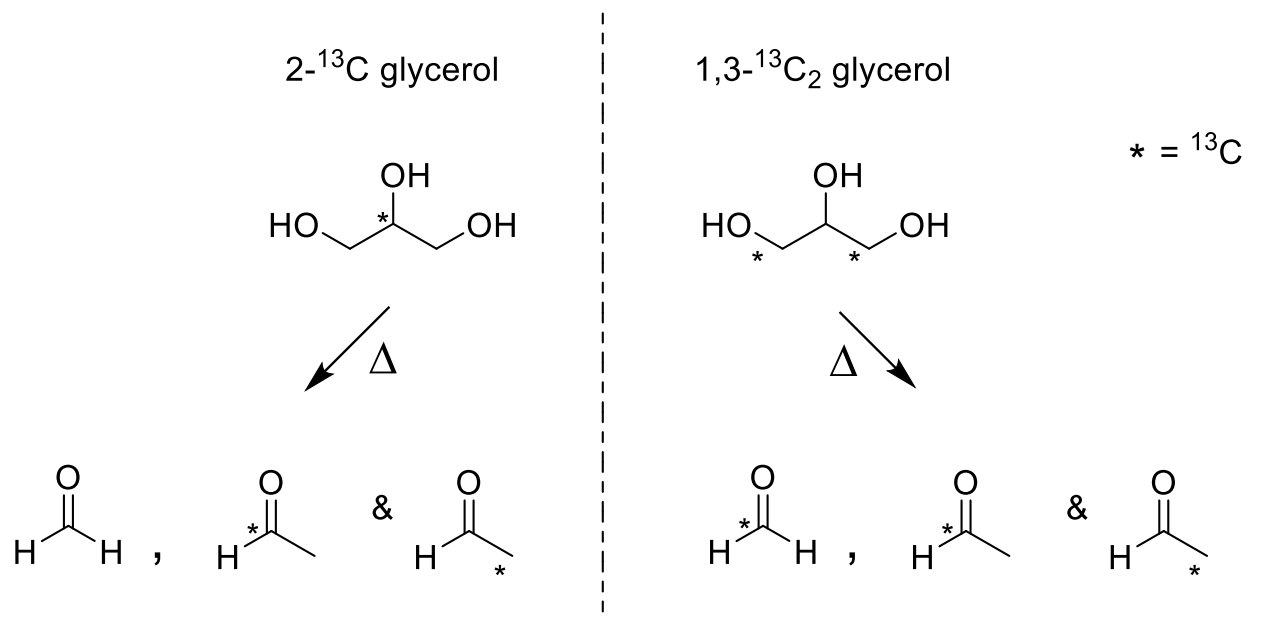

Scheme 1.5 - The Fate of ${ }^{13} \mathrm{C}$ during Glycerol Pyrolysis.

Unfortunately, the conditions of the experiments presented by Paine et al. are described with only minimal temperature/atmosphere details, and they note that several of the experiments discussed in their article were only performed one single time each. While their results are interesting, and while it is possible that the industrial affiliation of these authors has given me subconscious cause to read their work too critically, I do not believe 
that the results communicated by Paine et al. should be considered robust or generalizable to other experimental conditions or systems.

Laino et al. have published two recent works on pyrolysis of glycerol and propylene glycol: one entirely computational study in 2011 discussing glycerol dehydration, and a mixed computational and experimental study in 2012 discussing propylene glycol pyrolysis. ${ }^{67,75}$ In both of these studies the authors propose dehydration pathways which proceed through an oxirane intermediate: propylene oxide in the case of propylene glycol, and glycidol in the case of glycerol. In his 1904 publication, John Nef commented that glycerol was known to form glycidol at $240{ }^{\circ} \mathrm{C}$ in the presence of acids, and he reported that when heated at $450{ }^{\circ} \mathrm{C}$ pure glycidol rearranged into hydroxyacetone and its subsequent decomposition products, formaldehyde and acetaldehyde. ${ }^{44}$ The pathways involving the formation of these oxirane or epoxide rings proposed by Nef and Laino et al. are summarized in Scheme 1.6.

glycerol glycidol hydroxyacetone and 3-hydroxypropanal

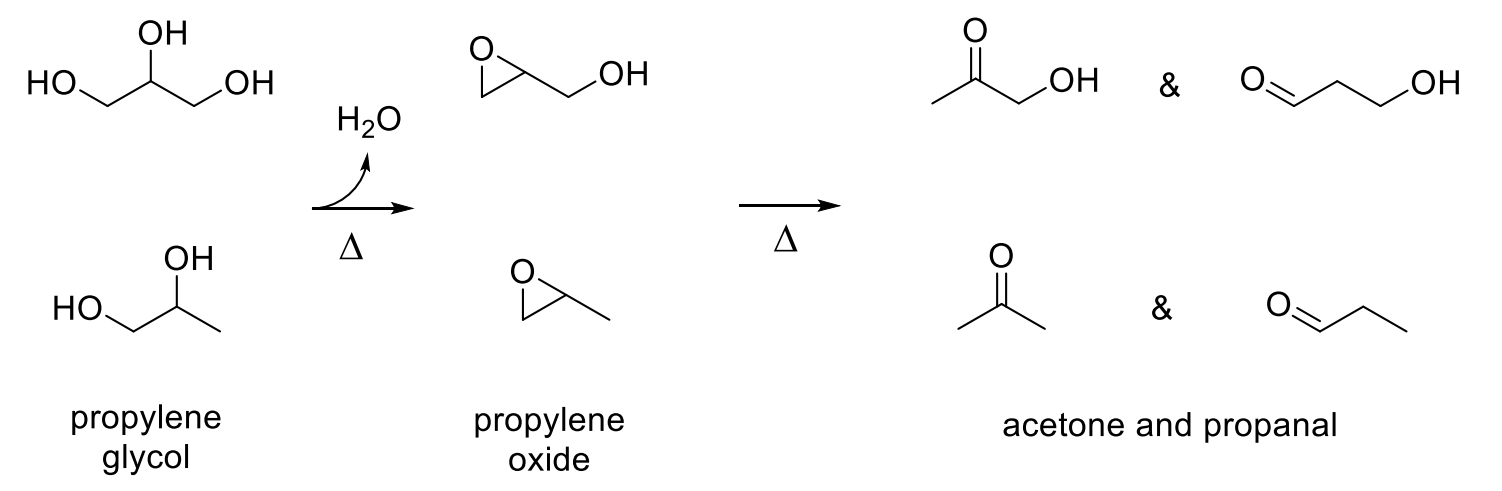

Scheme 1.6 - Oxirane Intermediate Formation in Glycerol and Propylene Glycol Dehydration. 
Nef did not know for certain that glycidol was an intermediate in the glycerol dehydration pathway he had observed, but he had no reason to doubt it considering his recovery of hydroxyacetone. However, the propylene glycol dehydration that he had observed generated propanal but did not generate any acetone, and Nef thereby reasoned that this pathway must not proceed through propylene oxide. The findings of Laino et al. may suggest that the specific conditions of Nef's experiments (e.g. temperature, atmostphere, heating surface, etc.) might have driven his results into only one of many possible directions. An article on the Mechanism of Glycerol Dehydration published in the Journal of Phyical Chemistry A in 2006 by Nimlos et al. discuss a number of potential pathways for neutral and protonated species of glycerol and glycidol - these authors appear to be unfamiliar with Nef's report of hydroxyacetone as a pyrolysis product of glycerol.

\subsubsection{Thermal Decomposition Studies of Propylene Glycol in Oxygenated Systems}

In 1985 an article was published by Rossiter et al. in Solar Energy Materials in which the authors described the liquid phase oxidative degradation of aqueous propylene glycol solutions at the low temperatures of $75-100{ }^{\circ} \mathrm{C}$ in the presence and absence of air and several metals. ${ }^{76}$ In this work the authors use ion chromatography to detect the formation of lactic, formic, and acetic acids, describing the processes of their formation rather simply as “...a complex process, involving a free-radical mechanism."

An article published by Díaz et al. from the University of California at Berkeley describes the most comprehensive study of the oxidative thermal degradation of propylene glycol. Writing to ChemSusChem in 2010, the authors found that propylene glycol decomposed in 
the gas phase at 400-600 $\mathrm{K}$ (approximately $130-330{ }^{\circ} \mathrm{C}$ ) into species including formaldehyde, acetaldehyde, and carbon dioxide. ${ }^{77}$ These experiments were conducted under a $10 \%$ oxygen, $90 \%$ helium atmosphere which was varied by further dilution with additional helium. In an interesting contrast to the results published by Nef, these authors observed the production of acetone but not propanal, and they speculate that formaldehyde and acetaldehyde are partial combustion products resulting from cleavage of the carboncarbon bond after $\mathrm{O}_{2}$ insertion and hydrogen abstraction; this abstraction is thought to result in hydroxyl radical elimination, leaving behind an unstable oxy-alkyl radical that terminates into formaldehyde, acetaldehyde, carbon dioxide, and water. Compared to the studies that have been discussed previously, the presence of oxygen in the system seems to have changed the pathway by which the molecule degrades and significantly lowered the temperature required for decomposition. This partial combustion pathway is summarized in Scheme 1.7. The authors noted that they observed no decomposition of glycerol under their experimental conditions.

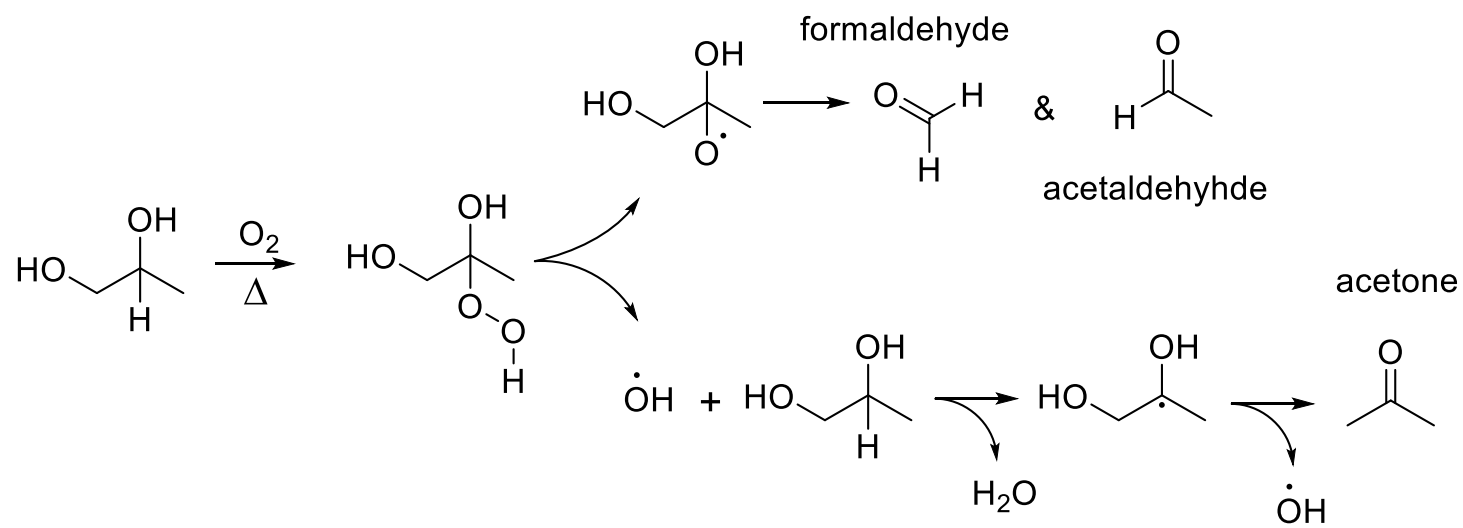

Scheme 1.7 - Propylene Glycol Combustion pathway proposed by Díaz et al. 


\subsection{Summary of Literature Discussion}

It has been understood that glycerol and propylene glycol are susceptible to degradation since their initial discoveries. Carl Scheele reported that boiling glycerol resulted in the formation of an acrid material in 1783, which was identified as acrolein and recognized as a dehydration product by Redtenbacher in 1843. Wurtz showed that propylene glycol could be oxidized into lactic acid in the same 1859 publication in which he announced its discovery. Nef and Sabatier each studied the thermal degradation of glycerol using different heating surfaces and observed different decomposition processes at different temperatures, suggesting that the speciation of products is dependent on the specific conditions of the experiment. Modern analytical and computational approaches have largely echoed the results described in these early studies and reflect rather favorably upon the pathways proposed by Sabatier and Nef a century ago. As we shall soon discuss, my own investigation into the thermal degradation of glycerol and propylene glycol that has been vaporized in air using an electronic cigarette may serve as further confirmation of their findings and proposals.

\subsection{Nuclear Magnetic Resonance Spectroscopy of Glycerol and Propylene Glycol}

My own investigations into the thermal decomposition of glycerol and propylene glycol have relied heavily upon Nuclear Magnetic Resonance (NMR) spectroscopy, a technique that utilizes radio-frequency electromagnetism to excite and observe an ensemble of nuclear spins as they decay back into ordered precession inside a strong magnetic field. Every nucleus of glycerol and propylene glycol can be observed using ${ }^{1} \mathrm{H},{ }^{13} \mathrm{C}$ and ${ }^{17} \mathrm{O}$ 
NMR spectroscopy with high-frequency spectrometers and high field-strength magnets. Having low natural abundance and magnetic receptivity, and being costly to enrich, ${ }^{17} \mathrm{O}$ is rarely the subject of observation in solution-state NMR experiments. In contrast, the utility of ${ }^{1} \mathrm{H}$ and ${ }^{13} \mathrm{C}$ NMR spectroscopy for the observation of small organic molecules is difficult to overstate. Samples can be observed at room temperature without perturbing the chemical equilibrium, and the signals detected using high-frequency spectrometers are rich in detail.

\subsubsection{The Utility of NMR Spectroscopy}

A properly-conducted ${ }^{1} \mathrm{H}$ NMR experiment will yield sharp resonance lines (peaks), wellresolved from one another along a frequency spectrum (horizontal axis), and having peak areas (signal intensity) proportional to the number of protons giving rise to each resonance. The fact that signal intensity is proportional to number of nuclei present makes NMR spectroscopy useful for quantitative molecular analysis. The natural-abundance of ${ }^{13} \mathrm{C}$ is much lower than ${ }^{1} \mathrm{H}$ on Earth, and the magnetic susceptibility is lower as well, making ${ }^{13} \mathrm{C}$ resonances more difficult to observe and quantify than protons.

Nuclear spins such as protons shift from their ideal Larmor precession frequency according to their specific chemical environment and electron density. In order to describe the position of a peak relative to the operating frequency of any specific spectrometer, the frequency spectrum is disguised as a "parts-per-million" (ppm) spectrum that can be generalized to any frequency/field-strength. Thus, the position of a resonance line, which was measured as a frequency, is discussed as a "chemical shift" from a reference position that has been designated as zero parts-per-million in a unit-less dimension. The resonance 
lines generated by nuclei which are coupled with adjacent NMR-active nuclei are split from a Lorentzian peak-shape into doublets, triplets, quartets, and further multiplets according to however many adjacent spins with which the nucleus is closely coupled. High-frequency spectrometers typically observe coupling between protons separated by three covalent bonds (i.e. bound to adjacent carbons), although other coupling events are often observed as well.

The shape, chemical shift, and relative intensity of a nuclear magnetic resonance signal offers sufficient information that an analyst can often solve simple structures of small organic molecules using only one-dimensional ${ }^{1} \mathrm{H}$ and ${ }^{13} \mathrm{C}$ experiments. Using twodimensional experiments such as Correlational spectroscopy (COSY), nuclear Overhauser effect spectroscopy (NOESY), Total Correlational spectroscopy (TOCSY), Heteronuclear Multiple-Bond Correlation (HMBC), and Heteronuclear Single-Quantum Coherence (HSQC) experiments, the structures of more complex molecules can be determined. Some of these two-dimensional experiments (HMBC and TOCSY in particular) allow an analyst to visualize distinct chemical species within samples of complex mixtures, separating signals that may not be fully resolved under one-dimensional analysis and unifying signals that may not appear coupled to a first approximation. The totality of information gathered by the above experiments makes this technique an extremely powerful tool in the molecular analysis of complex mixtures. The ${ }^{1} \mathrm{H}$ NMR spectrum of a sample containing a dozen chemical species might contain over a hundred unique resonance signals, even if the 
chemical species present in the ensemble are very similar in structure, polarity, molecular weight, and elemental composition.

\subsubsection{Experimental Considerations}

Although dilution in a solvent is not required to study propylene glycol and glycerol ${ }^{\mathrm{XI}}$ by NMR spectroscopy, it is usually desired for ${ }^{1} \mathrm{H}$ analysis. Deuterated water $\left(\mathrm{D}_{2} \mathrm{O}\right)$, methanol $\left(\mathrm{CD}_{3} \mathrm{OD}\right)$ or any other perdeuterated alcohol, dimethysulfoxide (DMSO-D 6 ), and dimethylformamide $\left(\mathrm{DMF}_{\mathrm{D}}\right)$ are suitable solvents for solution-state analysis. Solvents with an exchangeable deuterium atom, such as $\mathrm{D}_{2} \mathrm{O}$ or $\mathrm{CD}_{3} \mathrm{OD}$, will exchange the hydroxyl protons of glycerol and propylene glycol with bulk deuterium and thus are not useful for observing these important signals. Deuterated water affords the best resolution between the methylene and methyne proton resonances of propylene glycol and glycerol and offers the simplest and sharpest peak shapes. Glycerol is only sparingly soluble in aprotic organic solvents such as acetonitrile, acetone, and ethyl acetate. Addition of glycerol to a sample increases the sample viscosity and can have a dramatic effect on the chemical shift of all the peaks in a spectrum. All ${ }^{1} \mathrm{H}$ and ${ }^{13} \mathrm{C}$ spectra presented were collected at 599.80 and 150.82 MHz respectively from a Bruker Avance III $600 \mathrm{MHz}$ spectrometer and a 14 Tesla magnet. What little ${ }^{17} \mathrm{O}$ data is presented was collected at 54.24 MHz from a Bruker Avance III $400 \mathrm{MHz}$ spectrometer and a 9.4 Tesla magnet.

${ }^{\mathrm{XI}}$ Concentrated glycerol is too viscous to yield sharp resonance lines at $25^{\circ} \mathrm{C}$, but such an experiment is possible at $100{ }^{\circ} \mathrm{C}$ using a spectrometer with variable temperature control. 


\subsubsection{NMR Spectra of Glycerol}

\subsubsection{The ${ }^{1} H$ Spectrum of Glycerol}

The proton $\left({ }^{1} \mathrm{H}\right)$ spectrum of glycerol contains four resonance signals corresponding with the four non-equivalent protons found in the molecule, distinguished with subscripted Greek letters $\alpha, \beta, \gamma$, and $\delta$ in Figure 1.2.

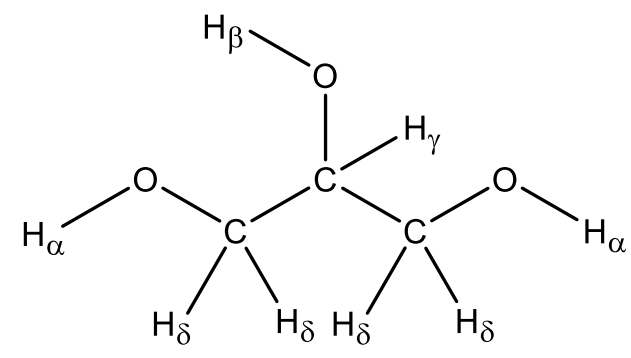

Figure 1.2 - Non-Equivalent Protons of Glycerol.

No proton in this molecule is further than two sigma bonds away from an oxygen - there are only (1) hydroxyl protons attached directly to oxygens and (2) alkyl protons attached to alcohol carbons. The alkyl resonances appear between 3.5-3.0 ppm, upfield (towards the right) from the hydroxyl protons which are located downfield (towards the left) near 4.5 ppm in DMSO-D 6 at $25{ }^{\circ} \mathrm{C}$, as shown in Figure 1.3. The small peak at $2.5 \mathrm{ppm}$ is the solvent peak, arising from the small amount of DMSO- ${ }_{5} \mathrm{H}$ present in commercially available DMSO- $\mathrm{D}_{6}$ that is labeled $99.9 \%$ deuterated. The chemical shifts in this spectrum are referenced to tetramethylsilane (TMS), appearing as the sharp peak at $0.0 \mathrm{ppm}$. 


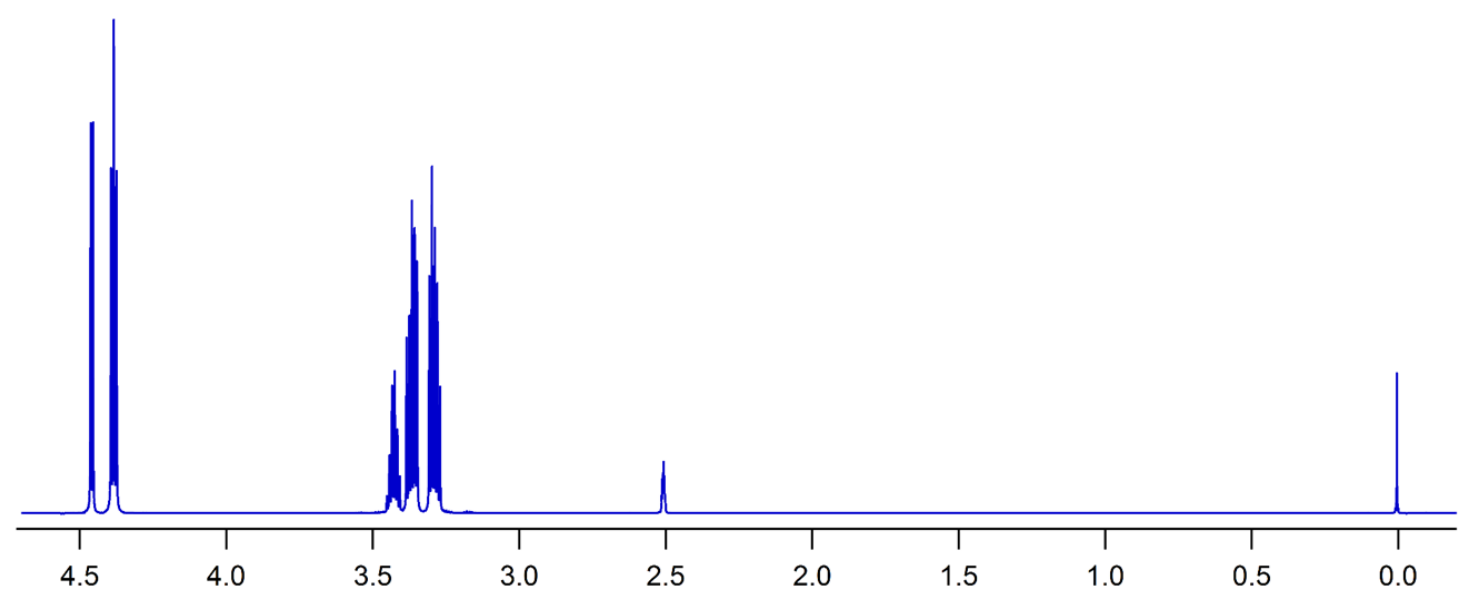

Figure 1.3 - The ${ }^{1} \mathrm{H}$ NMR Spectrum of Glycerol in DMSO-D 6 at $25^{\circ} \mathrm{C}$.

The ${ }^{1} \mathrm{H}$ resonances of glycerol are assigned as shown in Figure 1.4 according to their splitting and integration, using the same Greek-lettering scheme used in Figure 1.2 on expanded regions of the same spectrum shown in Figure 1.3.

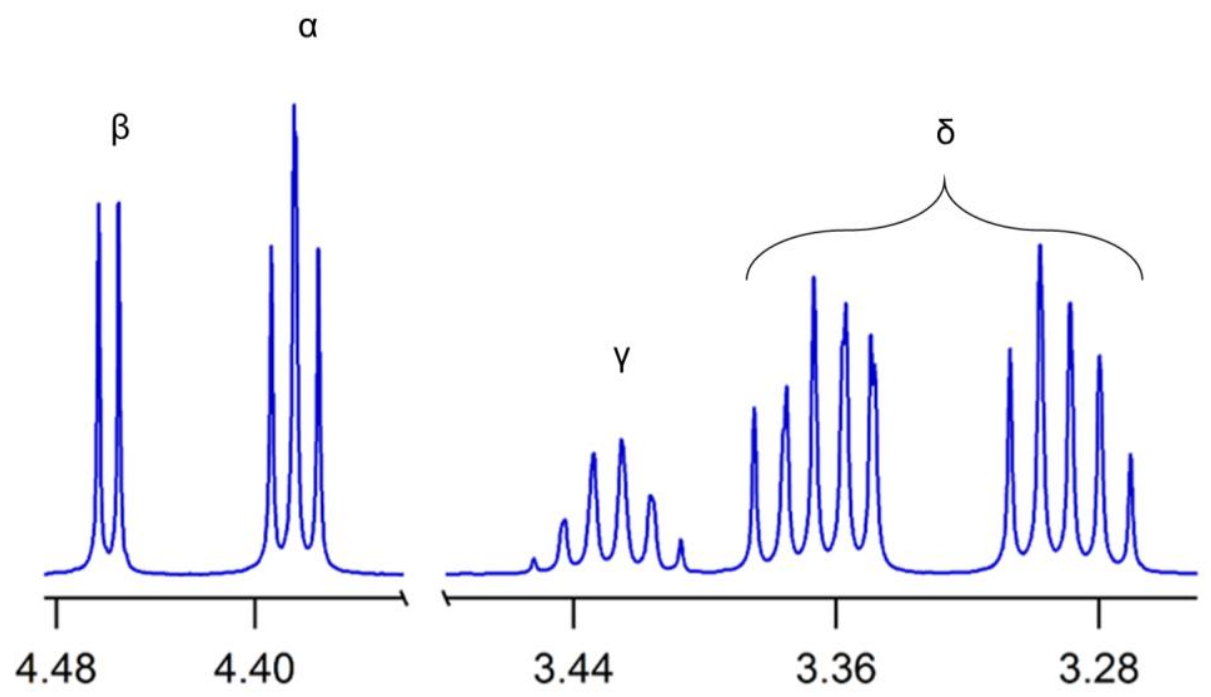

Figure 1.4 - Assignment of the ${ }^{1} \mathrm{H}$ Spectrum of Glycerol in DMSO-D 6 at $25{ }^{\circ} \mathrm{C}$. 
The $\alpha$-hydroxyl protons of glycerol resonate at $4.39 \mathrm{ppm}$ and are split into a doublet of doublets by the adjacent diastereotopic methylene protons. The $\beta$-hydroxyl, which is only split by the adjacent methyne proton, is a doublet shifted to $4.46 \mathrm{ppm}$. Like all exchangeable protons, the chemical shifts of these hydroxyl peaks vary strongly with changes in temperature and concentration. The methyne proton at $3.42 \mathrm{ppm}$ is split into a multiplet by the five adjacent protons. The diastereotopic methylene protons are split by the methyne proton and the $\alpha$-hydroxyls into a complex set of peaks "roofing" towards one another. While glycerol is fully resolved from the residual solvent peak at $2.5 \mathrm{ppm}$ in DMSO-D 6 , the water peak at $\sim 3.3$ ppm in DMSO-D 6 travels with changes in temperature and concentration, and it often overlaps with the methylene or methyne peaks. These peak shapes, shifts, and assignments are summarized in Table 1.1.

Table 1.1 - ${ }^{1} \mathrm{H}$ NMR Signals of Glycerol in DMSO-D 6 at $25^{\circ} \mathrm{C}$.

\begin{tabular}{|c|c|c|c|c|}
\hline Nucleus & Chemical Shift & Peak Shape & $\begin{array}{c}\left.\mathbf{1}^{\mathbf{H}}-{ }^{\mathbf{1}} \mathbf{H} \text { three-bond } \boldsymbol{(}^{\mathbf{J} J}\right) \\
\text { coupling }(\mathbf{H z})\end{array}$ & $\begin{array}{c}\text { Relative } \\
\text { Intensity }\end{array}$ \\
\hline $\boldsymbol{\alpha}$ & 4.39 & Doublet of doublets & $6.0,5.4$ & 2 \\
\hline $\boldsymbol{\beta}$ & 4.46 & Doublet & 4.9 & 1 \\
\hline $\boldsymbol{\gamma}$ & 3.42 & Multiplet & $*$ & 1 \\
\hline $\boldsymbol{\delta}$ & 3.32 & Multiplet & $*$ & 4 \\
\hline
\end{tabular}

\subsubsection{The ${ }^{13}$ C Spectrum of Glycerol}

The carbon $\left({ }^{13} \mathrm{C}\right)$ spectrum of glycerol contains two resonance signals corresponding with the two non-equivalent carbons found in the molecule, distinguished with subscripted Greek letters $\alpha$ and $\beta$ in Figure 1.5. 


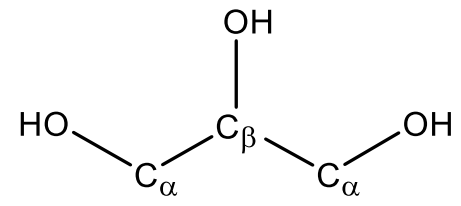

Figure 1.5 - Non-Equivalent Carbons of Glycerol.

The $\alpha$-carbons are primary $\left(1^{\circ}\right)$ alcohols with chemical shift of $63.3 \mathrm{ppm}$. The $\beta$-carbon is a secondary $\left(2^{\circ}\right)$ alcohol and is characteristically shifted downfield to $72.5 \mathrm{ppm}$. These resonances are shown without $\left\{{ }^{1} \mathrm{H}\right\}{ }^{13} \mathrm{C}$ decoupling in Figure 1.6.

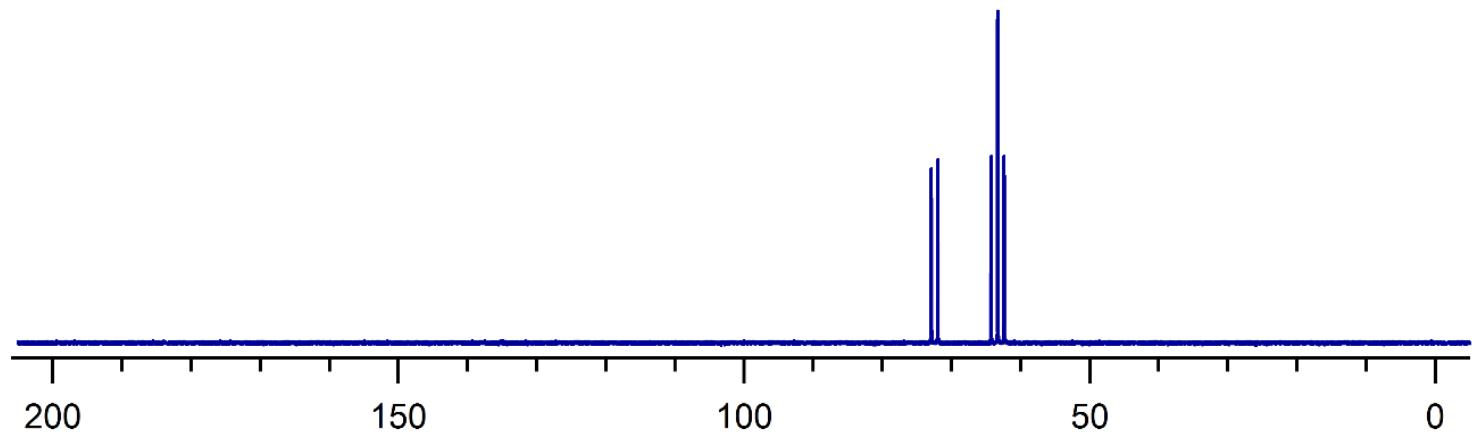

Figure 1.6 - The ${ }^{13} \mathrm{C}$ NMR Spectrum of Pure Glycerol at $100{ }^{\circ} \mathrm{C}$.

The $\alpha$-carbons are split into a triplet by their two bound methylene protons. The $\beta$-carbon is split into a doublet by the single bound methyne proton. The intensity of the $\alpha$-carbon triplet, corresponding to two equivalent nuclei, is approximately double the intensity of the $\beta$-carbon doublet by relative integration. These peak shapes, shifts, and assignments are detailed in Figure 1.7 and summarized in Table 1.2. 


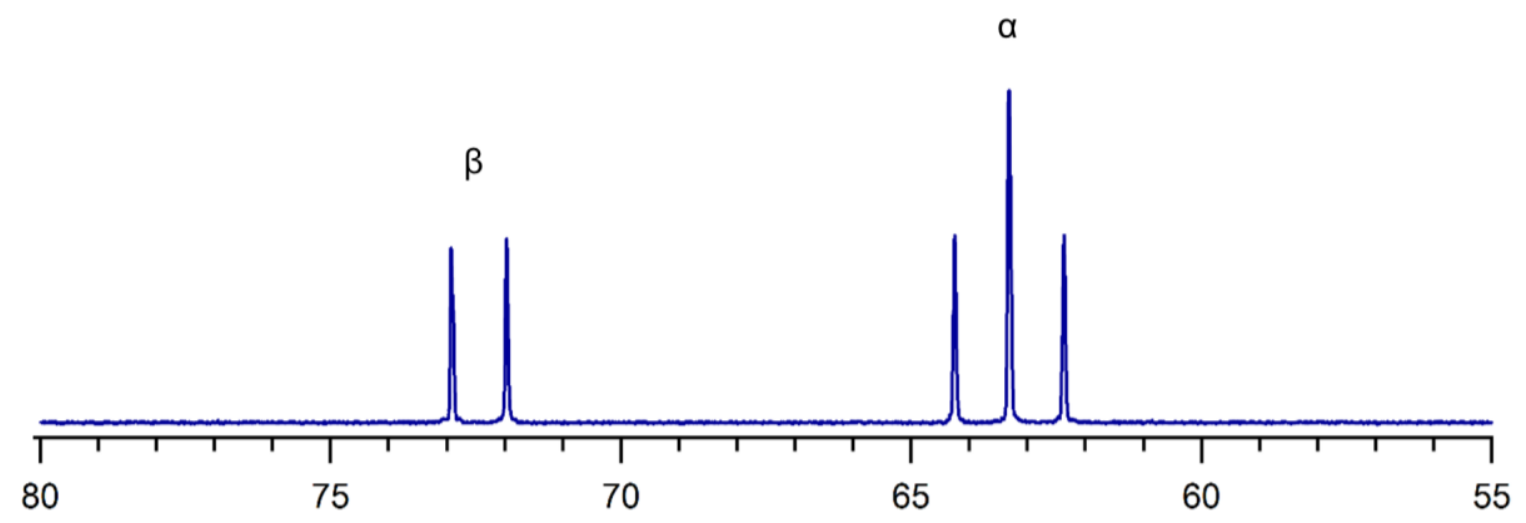

Figure 1.7 - Assignment of the ${ }^{13} \mathrm{C}$ NMR Spectrum of Pure Glycerol at $100{ }^{\circ} \mathrm{C}$.

Table 1.2 - ${ }^{13} \mathrm{C}$ NMR Signals of Glycerol.

\begin{tabular}{|c|c|c|c|c|}
\hline Nucleus & $\begin{array}{c}\text { Chemical } \\
\text { Shift }\end{array}$ & $\begin{array}{c}\text { Peak } \\
\text { Shape }\end{array}$ & $\begin{array}{c}\mathbf{H}^{\mathbf{1 3}} \mathbf{C} \text { single-bond }\left({ }^{1} \boldsymbol{J}\right) \\
\text { coupling }(\mathbf{H z})\end{array}$ & $\begin{array}{c}\text { Relative } \\
\text { Intensity }\end{array}$ \\
\hline $\boldsymbol{\alpha}$ & 63.3 & Triplet & 142 & 2 \\
\hline $\boldsymbol{\beta}$ & 72.5 & Doublet & 144 & 1 \\
\hline
\end{tabular}

\subsubsection{The ${ }^{17}$ O Spectrum of Glycerol}

The oxygen $\left({ }^{17} \mathrm{O}\right)$ spectrum of glycerol contains two resonance signals corresponding with the two non-equivalent oxygens found in the molecule, distinguished with subscripted Greek letters $\alpha$ and $\beta$ in Figure 1.8.

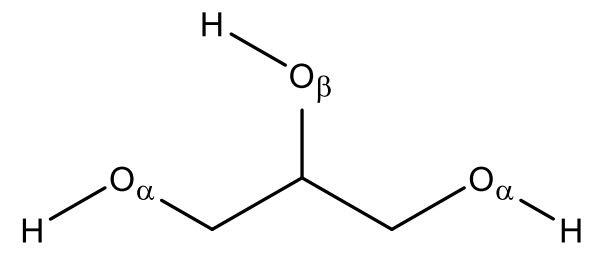

Figure 1.8 - Non-Equivalent Oxygens of Glycerol. 
The ${ }^{17} \mathrm{O}$ spectrum is shown in Figure 1.9. This spectrum was collected at $100{ }^{\circ} \mathrm{C}$ from a sample of concentrated glycerol (neat) in a $5 \mathrm{~mm}$ NMR tube.

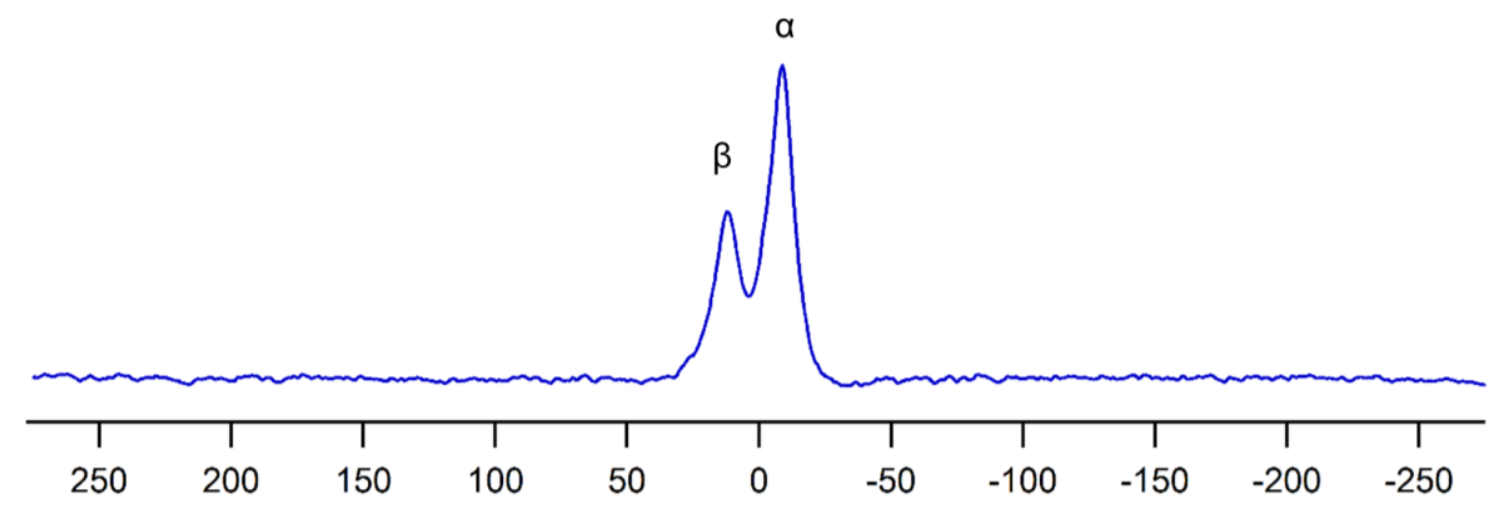

Figure 1.9 - The ${ }^{17} \mathrm{O}$ NMR Spectrum of Pure Glycerol at $100{ }^{\circ} \mathrm{C}$.

Though incompletely resolved, one can distinguish the ${ }^{17} \mathrm{O}$ resonance of the $1^{\circ}$ hydroxyls by its higher intensity relative to the smaller peak resulting from the lone $2^{\circ}$ hydroxyl moiety. The ${ }^{17} \mathrm{O}$ peak shifts and assignments of glycerol are summarized in Table 1.3.

Table 1.3 $-{ }^{17} \mathrm{O}$ NMR Signals of Glycerol.

\begin{tabular}{|c|c|c|}
\hline Nucleus & Chemical Shift & Relative Intensity \\
\hline $\boldsymbol{\alpha}$ & -9.0 & 2 \\
\hline $\boldsymbol{\beta}$ & 11.7 & 1 \\
\hline
\end{tabular}

\subsubsection{NMR Spectra of Propylene Glycol}

\subsubsection{The ${ }^{l}$ H Spectrum of Propylene Glycol}

The proton $\left({ }^{1} \mathrm{H}\right)$ spectrum of propylene glycol contains five resonance signals corresponding with the five non-equivalent protons found in the molecule, depicted with subscripted Greek letters $\alpha, \beta, \gamma, \delta$, and $\varepsilon$ in Figure 1.10. 


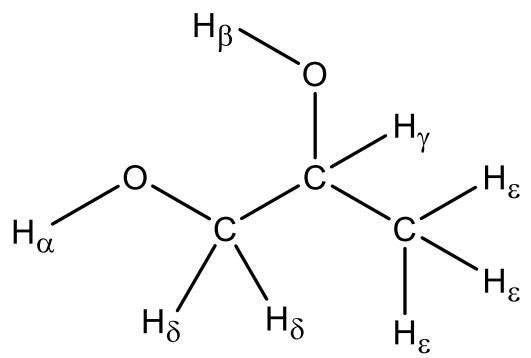

Figure 1.10 - Non-Equivalent Protons of Propylene Glycol.

The ${ }^{1} \mathrm{H}$ NMR spectrum of propylene glycol is shown in Figure 1.11. Unlike glycerol, propylene glycol contains a methyl carbon which is not covalently bound to an electronegative oxygen atom, visible in this spectrum as a tall peak at $1.0 \mathrm{ppm}$ split into a doublet by the adjacent methyne proton. This subtle structural alteration changes the electronic configuration of the molecule enough to shift the methyne proton downfield from the methyne of glycerol, and to shift the diastereotopic methylene protons upfield (although not fully resolving them) from the methylenes of glycerol. The chemical shift of the PG hydroxyl protons is nearly the same as in GL, but the orientation of the peaks is flipped - the $\alpha$-hydroxyl proton shifted further downfield than the $\beta$-hydroxyl proton of PG. The sharp peak visible at $\sim 3.3 \mathrm{ppm}$ is water, which sometimes overlaps with the methylene or methyne protons depending on concentration and temperature. The peaks arising from imperfectly-deuterated solvent and the internal shift reference (DMSO and TMS) are visible at $2.5 \mathrm{ppm}$ and $0.0 \mathrm{ppm}$, respectively. 


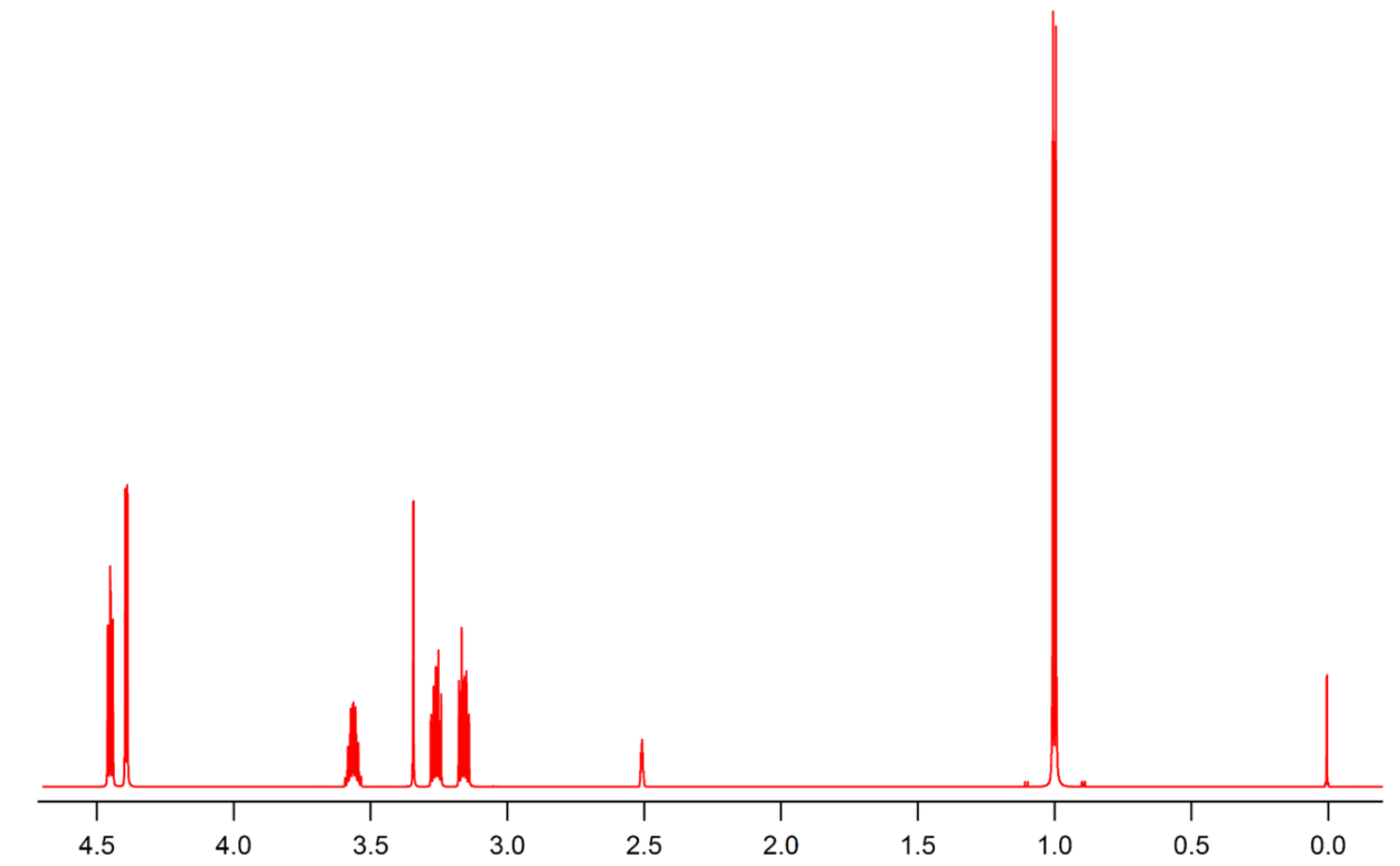

Figure 1.11 - The ${ }^{1} \mathrm{H}$ NMR Spectrum of Propylene Glycol in DMSO-D 6 at $25^{\circ} \mathrm{C}$.

The ${ }^{1} \mathrm{H}$ resonances of propylene glycol are assigned as shown in Figure 1.12 according to their splitting and integration, using the same Greek-lettering scheme used in Figure 1.10 on expanded regions of the same spectrum shown in Figure 1.11. 


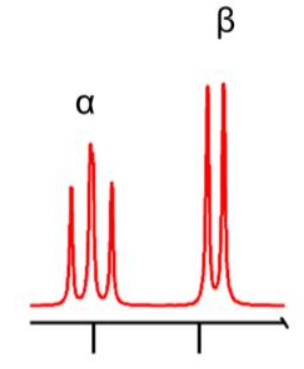

4.454 .40

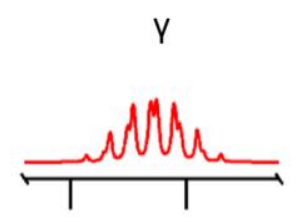

$3.60 \quad 3.55$

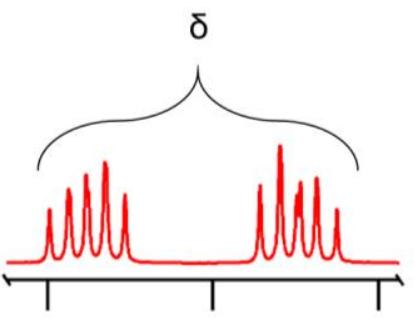

$3.28 \quad 3.20$

3.121 .00

Figure 1.12 - Assignment of the ${ }^{1} \mathrm{H}$ Spectrum of Propylene Glycol in DMSO-D 6 at $25{ }^{\circ} \mathrm{C}$.

The PG $\alpha$-hydroxyl proton resonance at $4.45 \mathrm{ppm}$ is split into a doublet of doublets by the adjacent diastereotopic methylene protons. The $\beta$-hydroxyl, which is split only by the single adjacent methyne proton, is a doublet shifted to $4.40 \mathrm{ppm}$. The methyne proton at $3.56 \mathrm{ppm}$ is split into a multiplet by the six adjacent protons. The diastereotopic methylene protons are split by the methyne proton and the $\alpha$-hydroxyl into a complex set of peaks "roofing" towards one another. The methyl proton at $1.0 \mathrm{ppm}$ is split only by the adjacent methyne proton into a doublet. These assignments are summarized in Table 1.4.

Table 1.4 - ${ }^{1} \mathrm{H}$ NMR Signals of Propylene Glycol in DMSO-D 6 at $25{ }^{\circ} \mathrm{C}$.

\begin{tabular}{|c|c|c|c|c|}
\hline Nucleus & Chemical Shift & Peak Shape & $\begin{array}{c}\mathbf{1}^{\mathbf{H}}-{ }^{\mathbf{1}} \mathbf{H} \text { three-bond }\left({ }^{\mathbf{3}} \boldsymbol{J}\right) \\
\text { coupling (Hz) }\end{array}$ & $\begin{array}{c}\text { Relative } \\
\text { Intensity }\end{array}$ \\
\hline $\boldsymbol{\alpha}$ & 4.45 & Doublet of doublets & $6.1,5.3$ & 1 \\
\hline $\boldsymbol{\beta}$ & 4.40 & Doublet & 4.5 & 1 \\
\hline $\boldsymbol{\gamma}$ & 3.56 & Multiplet & $*$ & 1 \\
\hline $\boldsymbol{\delta}$ & 3.21 & Multiplet & $*$ & 3 \\
\hline $\boldsymbol{\varepsilon}$ & 1.00 & Doublet & 6.4 & 3 \\
\hline
\end{tabular}




\subsubsection{The ${ }^{13}$ C Spectrum of Propylene Glycol}

The carbon $\left({ }^{13} \mathrm{C}\right)$ spectrum of propylene glycol contains three resonance signals corresponding with the three non-equivalent carbons found in the molecule, depicted with subscripted Greek letters $\alpha, \beta$, and $\gamma$ in Figure 1.13.

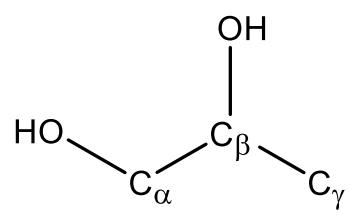

Figure 1.13 - Non-Equivalent Carbons of Propylene Glycol.

The $\alpha$-carbon is a primary $\left(1^{\circ}\right)$ alcohol with chemical shift of $67.7 \mathrm{ppm}$. The $\beta$-carbon is a secondary $\left(2^{\circ}\right)$ alcohol and is shifted to $68.2 \mathrm{ppm}$. The $\gamma$-carbon is an ordinary methyl that resonates at $19.0 \mathrm{ppm}$. These resonances are shown without $\left\{{ }^{1} \mathrm{H}\right\}{ }^{13} \mathrm{C}$ decoupling in Figure 1.14 .

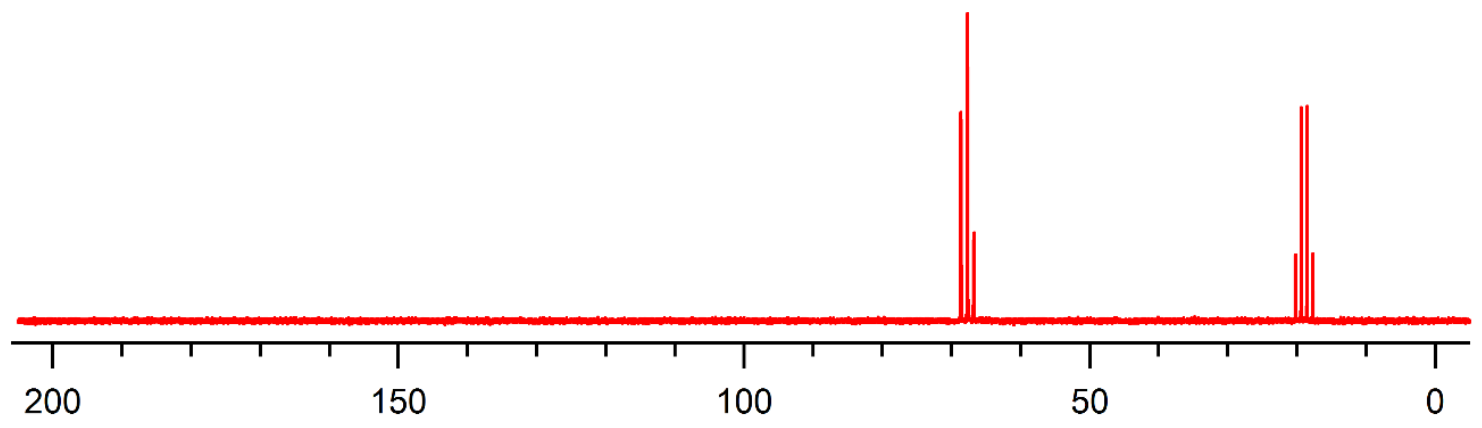

Figure 1.14 - The ${ }^{13} \mathrm{C}$ NMR Spectrum of Pure Propylene Glycol at $100{ }^{\circ} \mathrm{C}$. 
The $\alpha$-carbon is split into a triplet by the two bound methylene protons. The $\beta$-carbon is split into a doublet by the single bound methyne proton. The $\alpha$-carbon triplet and the $\beta$ carbon doublet happen to overlap under the conditions of the above experiment, giving the appearance of a lopsided triplet. ${ }^{\mathrm{XII}}$ The $\gamma$-carbon resonance is split into a quartet by the three bound methyl protons. These peak shapes, shifts, and assignments are detailed in Figure 1.15 and summarized in Table 1.5.

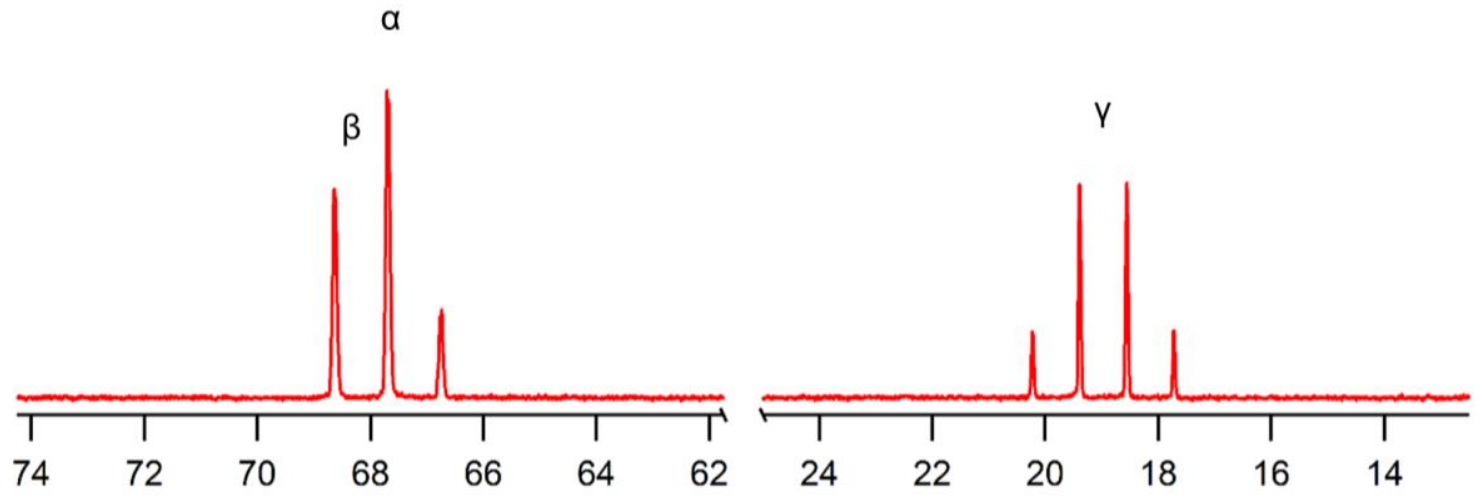

Figure 1.15 - Assignment of the ${ }^{13} \mathrm{C}$ NMR Spectrum of Pure Propylene Glycol at $100{ }^{\circ} \mathrm{C}$.

Table 1.5 $-{ }^{13} \mathrm{C}$ NMR Signals of Propylene Glycol.

\begin{tabular}{|c|c|c|c|c|}
\hline Nucleus & Chemical Shift & Peak Shape & $\begin{array}{c}{ }^{\mathbf{1}} \mathbf{H}-{ }^{\mathbf{1 3}} \mathbf{C} \text { single-bond }\left({ }^{\boldsymbol{I}} \boldsymbol{J}\right) \\
\text { coupling }(\mathbf{H z})\end{array}$ & $\begin{array}{c}\text { Relative } \\
\text { Intensity }\end{array}$ \\
\hline $\boldsymbol{\alpha}$ & 67.7 & Triplet & 142 & 1 \\
\hline $\boldsymbol{\beta}$ & 68.2 & Doublet & 140 & 1 \\
\hline $\boldsymbol{\gamma}$ & 19.0 & Quartet & 126 & 1 \\
\hline
\end{tabular}

XII The resolution between these ${ }^{13} \mathrm{C}$ peaks is even poorer when PG is diluted in DMSO-D . 


\subsubsection{The ${ }^{17}$ O Spectrum of Propylene Glycol}

The oxygen $\left({ }^{17} \mathrm{O}\right)$ spectrum of propylene glycol contains two resonance signals corresponding with the two non-equivalent oxygens found in the molecule, depicted with subscripted Greek letters $\alpha$ and $\beta$ in Figure 1.16.

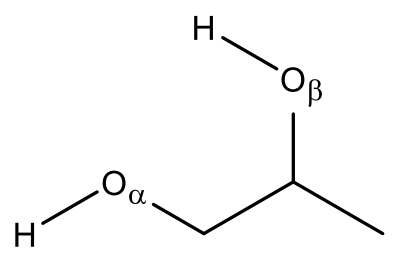

Figure 1.16 - Non-Equivalent Oxygens of Propylene Glycol.

The ${ }^{17} \mathrm{O}$ spectrum is shown in Figure 1.17. This spectrum was collected at $75{ }^{\circ} \mathrm{C}$ from a sample of concentrated propylene glycol (neat) in a $5 \mathrm{~mm}$ NMR tube.

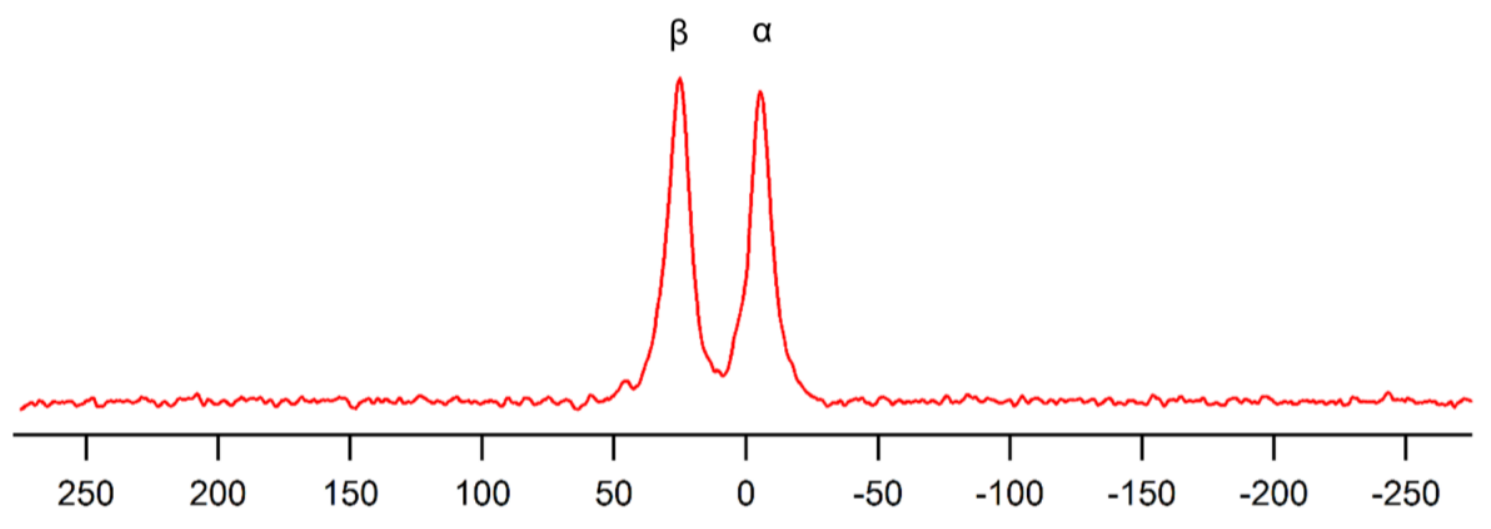

Figure 1.17 - The ${ }^{17} \mathrm{O}$ NMR Spectrum of Pure Propylene Glycol at $75{ }^{\circ} \mathrm{C}$. 
The ${ }^{17} \mathrm{O}$ peak shifts and assignments are summarized in Table 1.6.

Table 1.6 - ${ }^{17} \mathrm{O}$ NMR Signals of Propylene Glycol.

\begin{tabular}{|c|c|c|}
\hline Nucleus & Chemical Shift & Relative Intensity \\
\hline $\boldsymbol{\alpha}$ & -4.9 & 1 \\
\hline $\boldsymbol{\beta}$ & 25.5 & 1 \\
\hline
\end{tabular}

\subsubsection{NMR Spectra of Mixed Propylene Glycol and Glycerol}

Pure propylene glycol and glycerol have nearly identical room temperature concentrations (13.6 $\mathrm{mol} / \mathrm{L}$ and $13.7 \mathrm{~mol} / \mathrm{L}$, respectively). As such, mixtures of the liquids expressed as a percentage of the total number of molecules $(\% \mathrm{~mol})$ are roughly the same as mixtures expressed as a percentage of the total volume (\%vol). Glycerol forms strong intermolecular associations and has a high vaporization temperature; the addition of propylene glycol, which vaporizes more easily but does not form clouds of aerosols as thick as glycerol when used alone, acts to lower the viscosity and vaporization temperature of the liquid mixture. Most electronic cigarette liquids (e-liquids) utilize some mixture of glycerol and propylene glycol as co-solvents; stated simply, the two liquids vaporize nicely together. In order to approximate a simplified version of a typical e-liquid product, the ratio of $(50 / 50) \% \mathrm{~mol}$ was chosen as the standard mixture of propylene glycol and glycerol for this work - this mixture is referred to as (PG/GL) throughout this dissertation. This one-to-one molar ratio is convenient for quality assessment, being easy to analyze by relative integration of ${ }^{1} \mathrm{H}$ NMR signals. 
The ${ }^{1} \mathrm{H}$ NMR spectrum of (PG/GL) at $25^{\circ} \mathrm{C}$ in DMSO-D 6 contains the peaks described in sections 1.4.2.1 and 1.4.3.1, with incomplete resolution between the hydroxyl protons near $4.5 \mathrm{ppm}$ and the diastereotopic methylene protons near $3.3 \mathrm{ppm}$, even when observed near $600 \mathrm{MHz}$ in a 14 Tesla magnet. Fortunately, this field-strength is sufficient to fully-resolve the single methyne protons of PG and GL, appearing at $3.56 \mathrm{ppm}$ and $3.42 \mathrm{ppm}$ respectively in DMSO-D 6 at $25{ }^{\circ} \mathrm{C}$. These methyne protons are convenient for determining the molar equivalence of the two species by relative integration, and for quantitative determination relative to an internal standard. The incomplete resolution between PG and GL is demonstrated in Figure 1.18, where propylene glycol is plotted in red along the same spectrum upon which glycerol is plotted in blue.

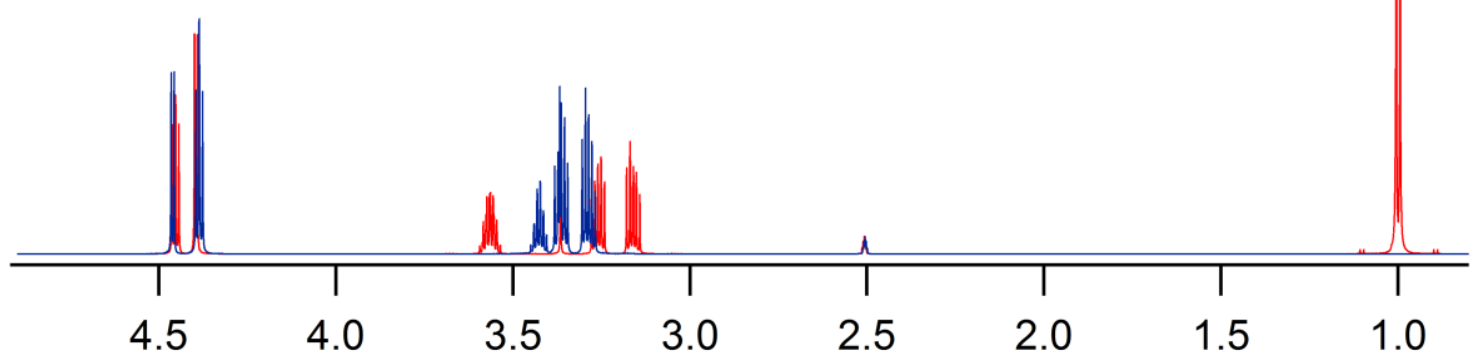

Figure 1.18 - Incomplete Resolution Between ${ }^{1} \mathrm{H}$ Resonances of PG (red) and GL (blue) Measured at 600 $\mathrm{MHz}$ in $\mathrm{DMSO}^{-\mathrm{D}_{6}}$ at $25^{\circ} \mathrm{C}$.

\subsubsection{NMR Spectra of Vaporized Propylene Glycol and Glycerol}

When (PG/GL) are pure and un-degraded, there are no peaks present in the region of the

${ }^{1} \mathrm{H}$ spectrum located downfield from the hydroxyl peaks near $4.5 \mathrm{ppm}$. A full view of the 
${ }^{1} \mathrm{H}$ spectrum is given in Figure 1.19, which contains a flat baseline between 10-5 ppm, throughout the downfield regions typically associated with resonances of aldehydes, aromatics, carboxylic acids, phenols, amides, alkenes, and other deshielded protons.

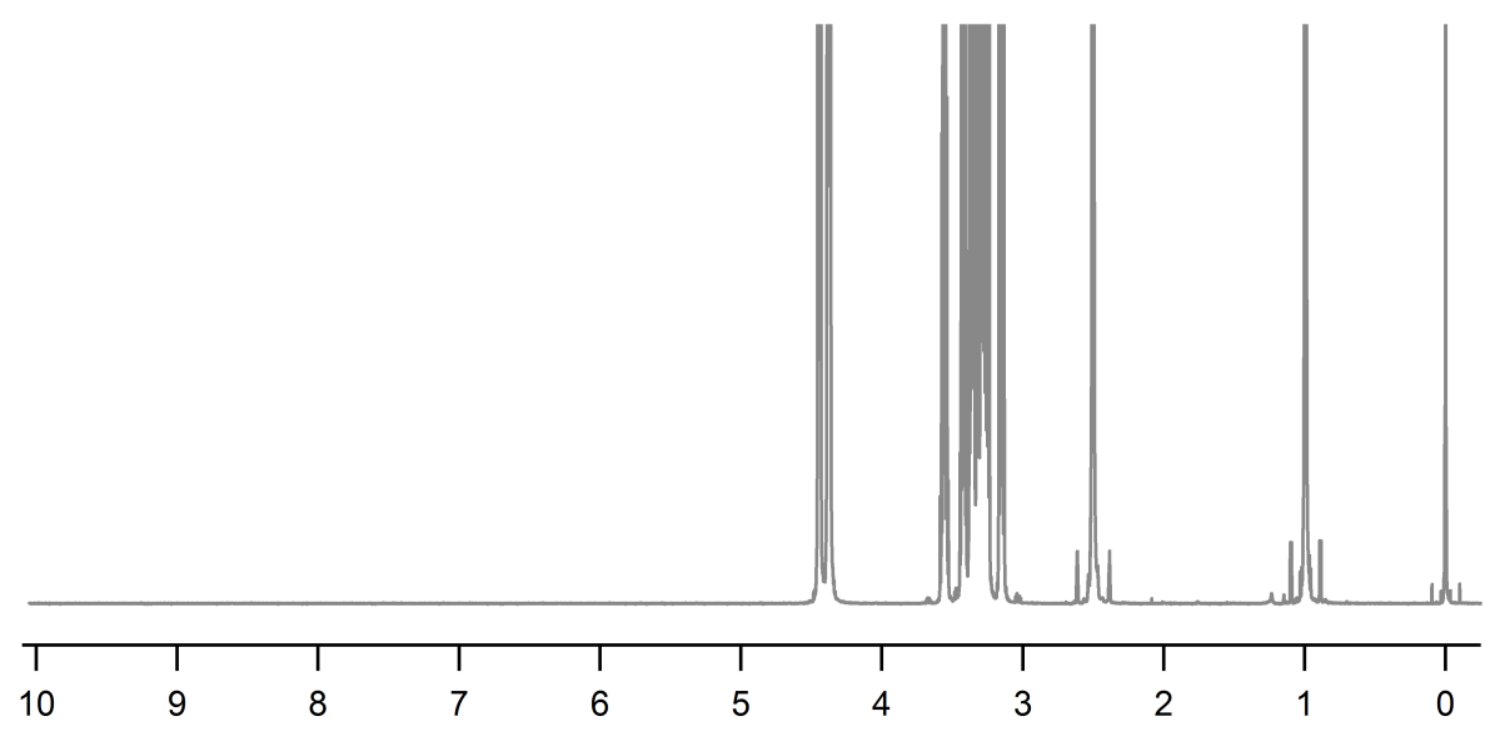

Figure 1.19 - Full ${ }^{1} \mathrm{H}$ NMR Spectrum of Pure (PG/GL) in DMSO-D 6 at $25^{\circ} \mathrm{C}$.

When a sample of (PG/GL) is vaporized using an electronic cigarette, new peaks can be seen throughout the ${ }^{1} \mathrm{H}$ NMR spectrum, indicating the formation of new molecules which were not present in the mixture before vaporization. This is typified in Figure 1.20, where a single puff of vaporized (PG/GL) is plotted in blue along with unvaporized (PG/GL) in grey. The peaks present in the blue spectrum which are absent in the grey spectrum correspond to new aldehyde, alkene, hydroxyl, acetyl, and other protons of chemical species formed by thermal decomposition. 


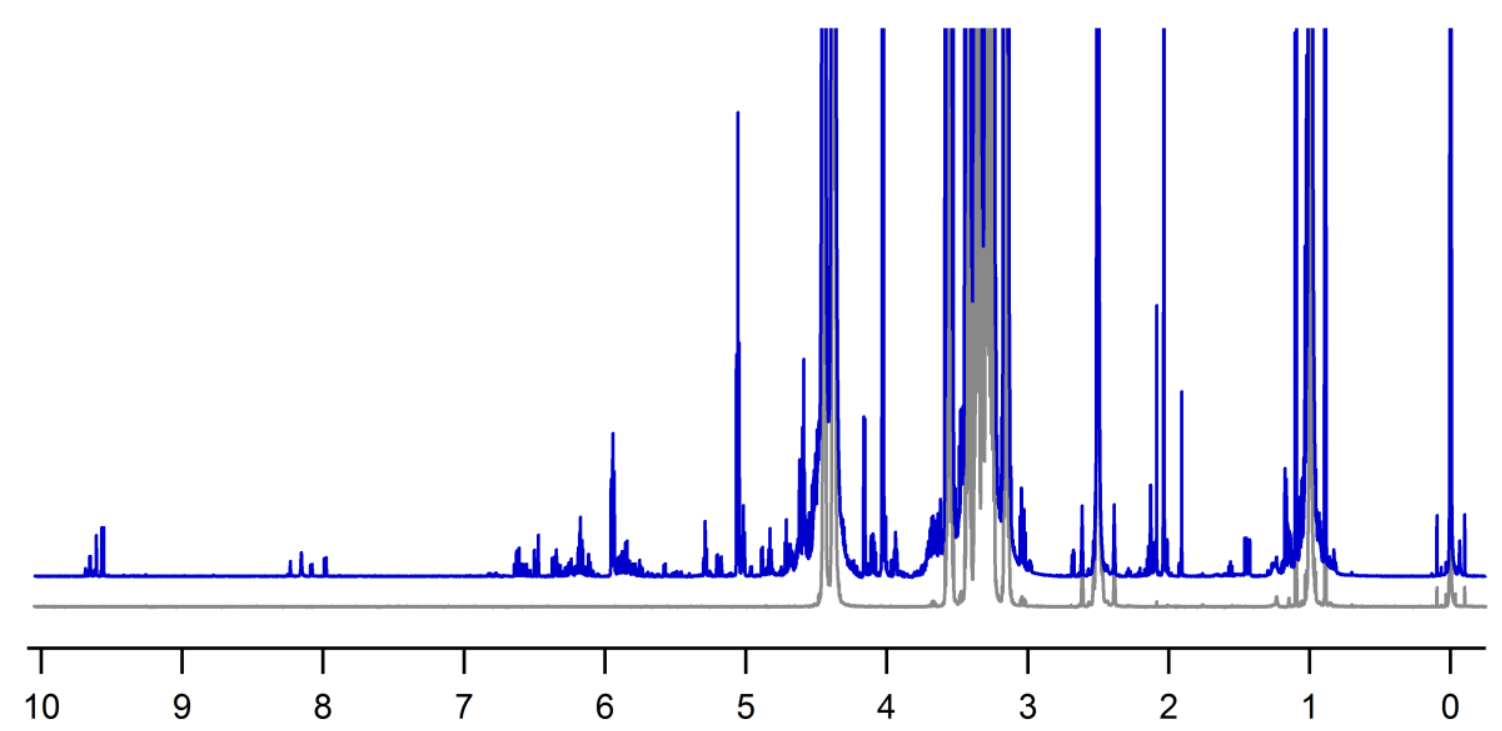

Figure 1.20 - The ${ }^{1} \mathrm{H}$ NMR Spectrum of Vaporized (PG/GL), Plotted in Blue, Contains Peaks Not Observed in Unvaporized (PG/GL), Plotted in Grey.

It is difficult to describe the fine detail present in the high-frequency spectra presented above while showing the full spectrum. As such, Figure 1.21 shows a very short region of the same spectra presented in Figure 1.20, running between 9.70-9.55 ppm in what might be described as the aldehyde region. This short section contains magnetic resonance signals corresponding to at least six decomposition products - aldehydes that have formed by dehydration or oxidation of propylene glycol or glycerol - that were not present in the sample of (PG/GL) before it was vaporized; among these products are acrolein, acetaldehyde, and propanal, which have been previously discussed in this chapter. 


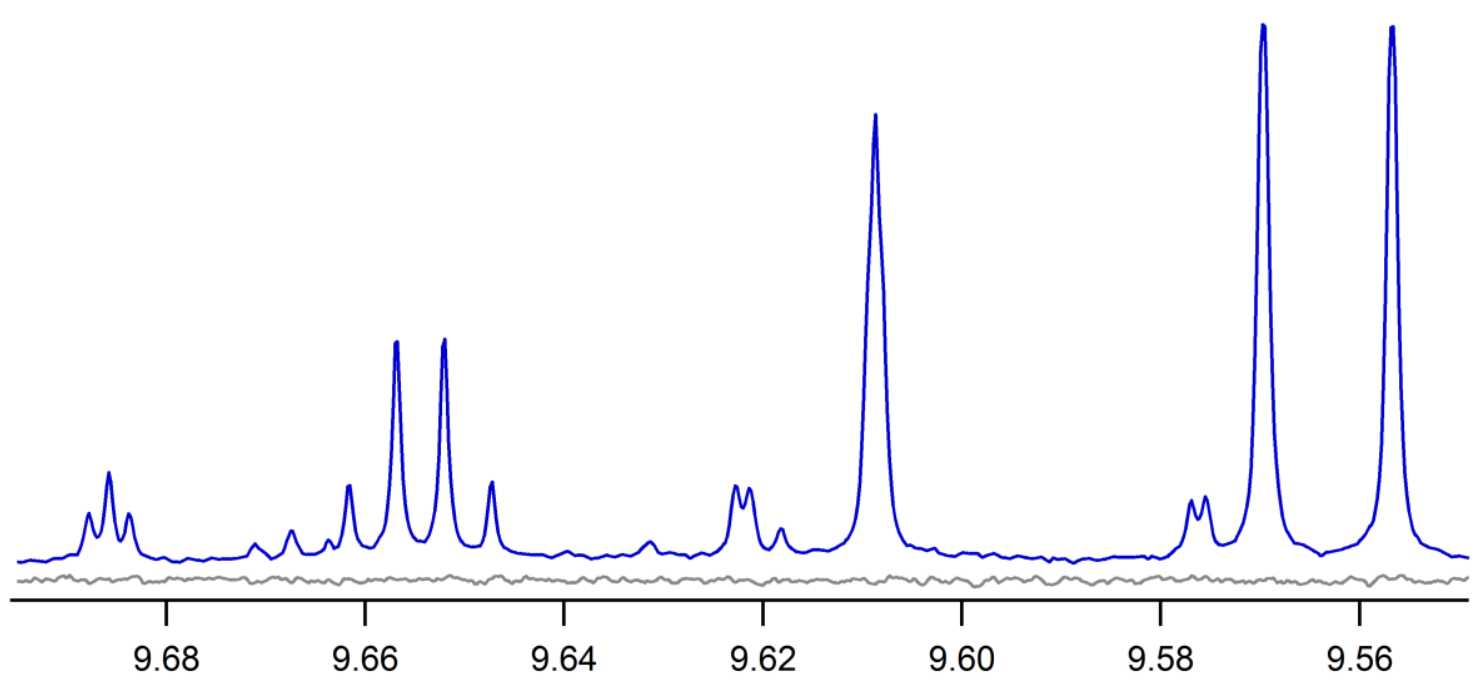

Figure 1.21 - The Aldehyde Region of Vaporized (PG/GL), Plotted in Blue, and Unvaporized (PG/GL), Plotted in Grey.

In this dissertation, products of the thermal decomposition of propylene glycol and glycerol (as visualized in the comparisons made between vaporized and unvaporized (PG/GL) demonstrated in Figures 1.20 and 1.21) are identified and investigated using NMR spectroscopy. Certain degradation products are quantified in samples collected under varying conditions by relative integration against an internal standard, and the reactivity of certain degradation products (and the subsequent reactions in which they participate) are investigated.

\subsection{Outline of Subsequent Chapters}

Chapter 2 is a brief introduction to electronic cigarettes, the devices that serve as the chemical reactors in my investigation of the aerobic thermal decomposition of glycerol and propylene glycol. 
Chapter 3 is the central work of this dissertation, where the degradation of e-liquids composed of mixed propylene glycol and glycerol (PG/GL) - is discussed in qualitative and quantitative terms.

Chapter 4 is a discussion of formaldehyde - a unique molecule that is generated by the thermal degradation of propylene glycol and glycerol, whose peculiar reactivity and difficulty of detection warrant an isolated description.

Chapter 5 is a short discussion of shisha - a smokable tobacco mixture of which glycerol is a major component.

Chapter 6 contains concluding thoughts and remarks about future work. 


\section{Electronic Cigarettes}

Electronic cigarettes (e-cigarettes) are a significant technological advancement in the consumer market of drug delivery by inhalation. While elegantly simple in concept and mechanism, modern devices have become remarkably sophisticated in design, composition, and operation. The subject has been well reviewed from regulatory and public health perspectives by a scientific community with good reasons to be concerned with nicotine addiction and critical of the tobacco industry. ${ }^{78-85}$ Considering our relatively early position in the evolution of e-cigarettes, I shall make no attempt at predicting what the future may hold for this still-emerging practice, nor do I intend to summarize what is at present known with certainty. Rather, this chapter discusses that information which might be useful in understanding how and why e-cigarettes are used, and how glycerol and propylene glycol may decompose when vaporized using an e-cigarette.

\subsection{How Electronic Cigarettes Work}

Electronic cigarettes use resistive heating to vaporize molecules that a user desires to inhale. An electric current supplied by a battery passes through a heating element typically a coil made of metal alloys such as kanthal or nichrome - which quickly heats to several hundred degrees when the device is actuated. The heating coil wraps around a wicking material that has been saturated with the drug of intended exposure, which may be stored within an integrated reservoir or added dropwise directly to the wick/coil by the user. In the case of nicotine, a potent stimulant isolated from tobacco leaves, the refined drug is diluted into a product typically referred to as electronic cigarette liquid (e-liquid) 
using glycerol and/or propylene glycol as solvents. Glycerol and propylene glycol, the molecules discussed at length in Chapter 1, are the most populous molecular species present in e-liquids. This dilute nicotine solution forms aerosolized droplets when vaporized, flowing into the lower respiratory system upon inhalation and delivering nicotine deep within the lungs exactly like traditional tobacco smoke; from the lungs the drug moves rapidly to the brain where its effects manifest. The drug uptake mechanism utilized by e-cigarettes is directly analogous to smoking - a delivery method that has been well characterized after decades of investigation - but the molecular composition of the inhaled material is much less diverse; the drug is more refined than plant material, and the heating mechanism is under electronic control.

\subsection{The Rise and Regulation of Electronic Cigarettes}

The rapid development and proliferation of e-cigarettes is easy to understand considering the testimony provided by literally every e-cigarette user that I have ever conversed with; they all believe that e-cigarettes have helped them stop smoking traditional cigarettes. The fact that consumers like a device designed to deliver nicotine to their lungs should come as no surprise to anyone. Informed opinions about the safety and utility of e-cigarettes as a smoking-cessation device will require long-term studies that will almost certainly face criticism from industrial interests. Regardless of whatever utility e-cigarettes may have as a smoking-cessation product, a casual investigation reveals that these devices are used recreationally (even competitively) and that their drug delivery applications extend beyond nicotine. 
On May $5^{\text {th }}, 2016$ the US Food and Drug Administration (FDA) announced that it had deemed e-cigarettes to fall under the regulatory scope of the 2009 Tobacco Control Act. Under the FDA proposal e-cigarette products are regarded as tobacco products, and as such, producers of e-cigarettes and e-liquids must register with FDA, submit to inspections, and disclose manufacturing processes and product details. Sales to persons younger than 18 years old will also be restricted under the announced regulations. The State of California has recently announced that under new regulation persons under the age of 21 will be barred from purchasing tobacco products including e-cigarettes.

\subsection{Smoking vs. Vaping}

Smoke is not a particularly well-defined concept - it is typically understood as a mixture of vapors and aerosols emitted during the combustion of organic material. The definition of smoke therefore depends on the definitions of a number of other complex terms including combustion, which evokes the high temperature recombination of carbon from organic material with oxygen from air. The word smoke is not generally used to describe the exhaust of complete combustion to the terminal products carbon dioxide and water; rather, it is usually employed to describe complex mixtures of chemical species emitted at lower temperatures by partial combustion. As was discussed in Chapter 1, glycerol and propylene glycol, the liquids which form the base of e-liquid, are susceptible to the type of oxidation reactions one might term partial combustion at temperatures near their boiling points. 
The e-cigarette community has adopted the word vapor to describe suspended e-liquid droplets. While the process that leads to the initial formation of aerosolized e-liquid might rightly be described as vaporization, the puff that is exhaled by an e-cigarette user is a mixture of organic aerosols and gases which more closely resembles smoke than anything else. Indeed, the clouds of aerosolized propylene glycol emitted by commercial smoke machines in theatrical productions are referred to as smoke. Efforts to distinguish ecigarettes from traditional cigarettes have resulted in the development of the terms vape, vaping, and vapers to be used instead of smoke, smoking, and smokers. Thus, the smoke shop has become the vape shop; where vaping crowds of vapers blow thick clouds of vape and compare vaporizers. I believe that this terminology has resulted in the common misconception that the cloud of material exhaled by an e-cigarette user is composed primarily of water vapor - a claim that I have even read on product labels. I find it remarkable that people are willing to believe the human lung can turn e-liquid into water.

\subsection{Typical Vaping Behavior}

I do not believe that typical vaping behavior can be precisely defined at present, although studies have attempted to ascertain the average e-cigarette puff duration, reporting values

of 3-4 seconds to displace $\sim 50 \mathrm{~mL}$ of volume. ${ }^{86-88}$ The broad diversity of e-cigarette devices (discussed in Section 2.5), which extends down to the composition and design of the atomizing heating element, makes it difficult to compare behavioral metrics like operating power or puff duration between individual users or devices. Based on my observations, e-cigarette users tend to experiment with different power levels and devices, and may change their behaviors and habits over time as they become more experienced. 
The diversity of e-liquid composition (varying in concentration of PG, GL, nicotine, and flavorants) adds further disorder to the set of variables relevant to thermal decomposition. It is presumptuous to draw a strong correlation between e-liquid decomposition and behavioral variables like operating power and puff duration without the specific context of these other important variables, which themselves may have an impact on the direction and extent of decomposition.

\subsection{Electronic Cigarette Device Diversity}

The consumer e-cigarette market might be broadly summarized as consisting of (1) disposable devices marketed towards casual consumers, (2) reusable devices, sold preassembled or as components, marketed towards regular users, and (3) highly modifiable reusable devices, sold as components, marketed towards technical users. Each of these loose classes contains a wide array of products differing in operating power, material composition, and design. Few objective statements can be made about e-cigarettes in general considering the plurality of devices unified under the term. Given this context, the results discussed subsequently within this work should be regarded as among the chemistry that can occur when propylene glycol and glycerol are vaporized using an e-cigarette, not as the chemistry that will certainly occur using any e-cigarette.

\subsubsection{The E-Liquid Reservoir and Heating Element}

Many reusable e-cigarettes employ an onboard reservoir filled with e-liquid, which saturates the wick and is in direct contact with the heating coil surface. These e-liquid tanks are sometimes referred to as cartomizers (presumably a combination of cartridge and 
atomizer) and can be understood as a reservoir combined with a heating element and a mouthpiece. This component typically threads onto a rechargeable battery, which may supply a fixed or variable voltage. Cartomizers are often modular in design, allowing users to replace a corrupted heating element or to vary the coil resistance. Devices are typically constructed of both plastics and metals, utilizing silicone rubber gaskets and a variety of wicking materials, while heating elements may employ one or two coils. This diversity of design and composition is an important consideration in characterizing the thermal degradation of glycerol and propylene glycol by e-cigarettes. Review of the relevant chemical literature (see Chapter 1) suggests that the specific characteristics of the heating surface are important in determining the temperature and pathway of the pyrolysis and combustion reactions that these molecules might undergo. The composition and condition of the heating element surface may be more important in determining the extent and direction of degradation than other variables, including temperature and residency time. Individual devices that appear functionally equivalent or even identical may generate different degradation profiles when operating at the same power level.

\subsubsection{Cartomizer Comparison}

Newer and more sophisticated devices, which may be manufactured in a more controlled and standardized process, tend to result in less degradation than older devices that were more crudely manufactured. This trend is typified in Figure 2.1, showing a comparison of vaporized propylene glycol and glycerol collected from four different consumer e-cigarette cartomizer devices. 


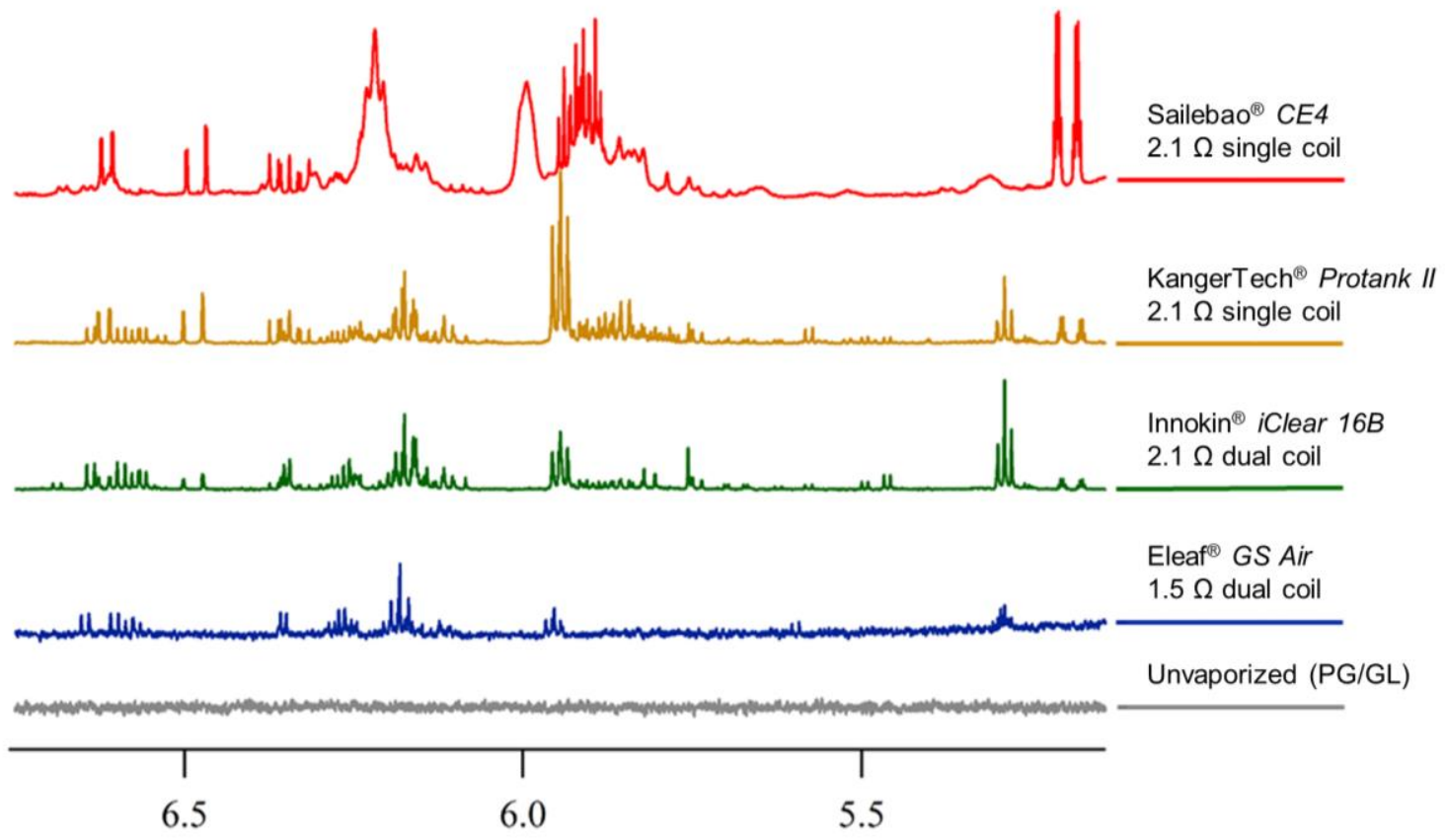

Figure 2.1 - Comparison of (PG/GL) Decomposition by Cartomizers of Different Design and Manufacture.

Figure 2.1 shows a short section of the ${ }^{1} \mathrm{H}$ NMR spectra of vaporized samples of (PG/GL) collected under varying conditions into $\mathrm{DMSO}_{6}$ and analyzed at $600 \mathrm{MHz}$, plotted against an unvaporized sample (shown on bottom in grey) demonstrating the flat baseline of the pure compounds within this region of the spectrum. The red spectrum, shown on top, was taken from a sample vaporized using an inexpensive Sailebao ${ }^{\circledR} C E 4$ cartomizer $^{\mathrm{XIII}}$ and shows the highest abundance and diversity of degradation products. Samples collected from more sophisticated devices typically produce spectra containing fewer peaks of lower

XIII Now considered an outdated design, the CE4 cartomizers discussed in this work were purchased as a component of a "Starter Kit" in 2014 and are still widely available. 
intensity, as demonstrated by the KangerTech ${ }^{\circledR}$ Protank-II and the Innokin ${ }^{\circledR}$ iClear $16 B$ plotted in the middle in orange and green respectively. Expensive modern devices such as the Eleaf® GS Air might produce vaporized samples containing little or no evidence of decomposition in their spectra, as exemplified by the small peaks growing out of the noisy baseline of the spectrum plotted in blue near the bottom.

\subsubsection{Replacement Coil Comparison}

Individual heating elements of the same design and manufacture, identical in appearance and packaged together as a batch of equivalent replacement units, can demonstrate wide variation in the abundance and profile of decomposition products observed in samples collected under identical experimental conditions. This is demonstrated in Figure 2.2, summarizing results from vaporized samples of (PG/GL) collected under fixed conditions from a KangerTech ${ }^{\circledR}$ Protank-II cartomizer, varying only the replaceable single-coil heating element installed in the cartomizer. Three single-puff samples were collected at a power setting of $10.0 \mathrm{~W}$ from eight different replacement coils, three of which were labeled $2.2 \Omega$ resistance by the manufacturer, while the other five were labeled $1.8 \Omega$ resistance. 


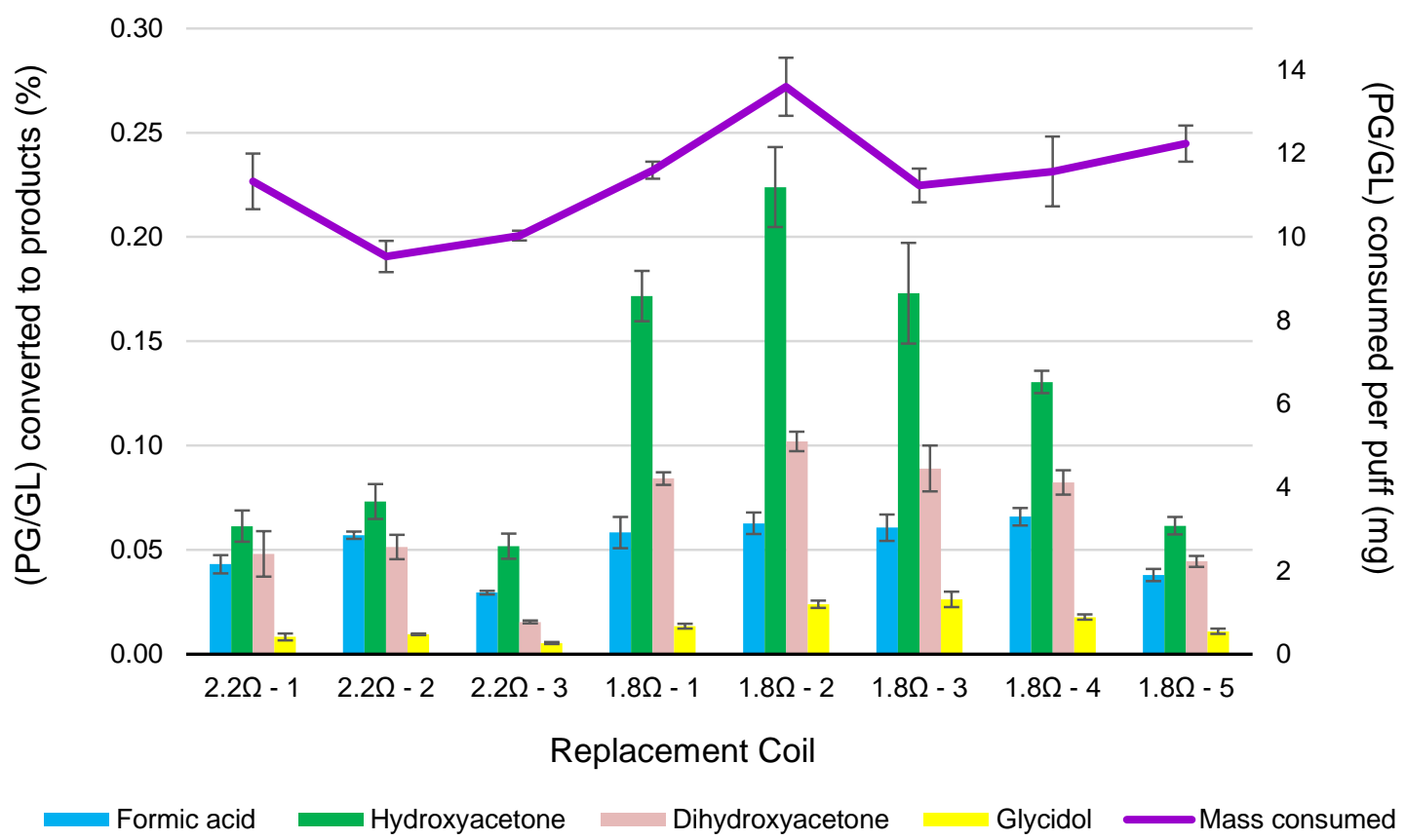

Figure 2.2 - Replacement Coil Comparison.

The intensity of magnetic resonance signals from several degradation products were compared by relative integration to the intensity of un-degraded (PG/GL) peaks, allowing for the qualitative expression of product concentrations; if only one species were present, such a ratio could be regarded as a simple percentage of conversion. Thus, for the sake of simplicity, the sample concentration of the thermal degradation products formic acid, hydroxyacetone, dihydroxyacetone, and glycidol are plotted as percentages of the concentration of left-over (PG/GL) against the left vertical axis. The cartomizer was carefully weighed in triplicate on an analytical balance before and after each puff in order to determine the mass of (PG/GL) consumed per puff, which is plotted as a purple line against the right vertical axis. Error bars depict the uncertainty in the mean $\left(\delta \chi_{\mathrm{avg}}\right)$ of the triplicate measurements. From the data summarized in Figure 2.2 it may be tempting to 
speculate that the lower resistance $1.8 \Omega$ coils consume more (PG/GL) per puff and create more degradation products than the cosmetically identical $2.2 \Omega$ coils, which presumably take slightly longer to heat; however, the results presented in Figure 2.2 draw from far too few samples and far too little data to justify such a sweeping conclusion.

\subsection{Reports of Degradation by Electronic Cigarettes in the Literature}

The degradation of e-liquids - the dilute solution of nicotine and flavorants dissolved in some mixture of propylene glycol and glycerol - has become an area of increased study over the past decade. So far as I can tell, the first study published on the subject of e-liquid degradation appeared in the Journal of Analytical and Applied Pyrolysis in $2007 .{ }^{74}$ In this work, researchers at Philip Morris USA used prototypes of their Accord ${ }^{\mathrm{TM}}$ electronically heated cigarette system that had been loaded with ${ }^{13} \mathrm{C}$-labeled glycerol, tracking the fate of the $\alpha$ - and $\beta$-carbons as glycerol decomposed into acrolein, formaldehyde, and acetaldehyde under the typical operating conditions of their device. The samples analyzed in this study were collected using a smoking machine, which smoked eight puffs by the ISO 3308 method of cigarette smoking ( $35 \mathrm{~mL}$ puff volume, 2 second puff duration, 60 second puff intervals). These eight-puff samples were filtered through Millipore $3 \mu \mathrm{m}$ nitrocellulose pads, and the filtered smoke was analyzed by GC/MS. This study, authored by Paine et al., is discussed in greater detail in Chapter 1, Section 2.1.2.

Most other published reports on the thermal decomposition of propylene glycol and glycerol by e-cigarettes have similarly focused on the detection and quantification of the above-mentioned aldehyde compounds. ${ }^{89-95}$ These studies generally use smoking 
machines to produce e-liquid vapor/aerosol that is pulled through 2,4dinitrophenylhydrazine (DNPH)-coated silica gel, which forms adducts with the carbonyl compounds that can be quantified chromatographically. It is generally agreed upon that increased e-cigarette operating temperatures are correlated with increased abundance of carbonyl decomposition products present in the resultant vapor. Other studies on aerosolized e-liquids have detected metal particles by inductively coupled plasma atomic emissions spectroscopy and free radicals by electron paramagnetic resonance spectroscopy. ${ }^{96,97}$ To my knowledge, no published study exists utilizing NMR spectroscopy to analyze samples of vaporized e-liquid for thermal degradation products, though the technique has been used to study the composition of unvaporized e-liquids. ${ }^{98}$ 


\section{Electronic Cigarette Liquids}

Free-base nicotine is a nonviscous liquid with a boiling temperature of $247{ }^{\circ} \mathrm{C}$ at atmospheric pressure, but the drug is not routinely vaporized and inhaled in pure form. Propylene glycol and glycerol are used to dilute nicotine into a viscous solution of lower potency which can be handled and inhaled; these propanols, which are generally recognized as safe for moderate oral consumption, form the base of electronic cigarette liquids (e-liquids) as mixed carrier solvents. E-liquid mixtures are countless and diverse, varying in the ratio of propylene glycol to glycerol and in the concentration of nicotine and the bouquet of molecules used to flavor the mixture. Based on my observations, e-liquid consumers tend to experiment with an assortment of products and qualitatively determine which mixtures and flavors they like best; experienced consumers sometimes mix their own e-liquids.

\subsection{Electronic Cigarette Liquid Composition}

E-liquid is a mixture of propylene glycol and glycerol containing some water, nicotine, flavorants, and other small molecules. One can find e-liquids that do not contain nicotine, but most products contain approximately $1-3 \%$ of the liquid alkaloid by volume. Propylene glycol is mixed with glycerol to lower the boiling point and viscosity of the e-liquid, but is not always used in products or applications where high viscosity is desired. Propylene glycol and glycerol are both hygroscopic, ${ }^{1,2}$ thus e-liquid will always contain at least a small amount of water, whether by design or happenstance. Small organic molecules including benzaldehyde, vanillin, and cinnamaldehyde are currently used in products at 
undisclosed concentrations without any type of testing or oversight under the assumption that molecules deemed safe for moderate ingestion are also stable to vaporization and safe for inhalation. Under regulations recently announced by the US Food and Drug Administration, producers of e-liquids will be required to disclose product details, and some uniformity may result.

\subsubsection{Simplified Electronic Cigarette Liquid}

In order to begin this investigation into the thermal decomposition of e-liquid, the mixture studied was simplified to a one-to-one molar ratio of pure propylene glycol and glycerol, which are the most populous molecules in e-liquid. This 50/50 mixture is designated (PG/GL) throughout this dissertation. The decision to focus on the solvents and exclude nicotine or other additives from this investigation almost certainly simplifies the chemistry, given that the extent and direction of degradation may be susceptible to the presence of base or acid which may act as a catalyst or reactant. Careful reading of Chapter 1 should affirm that this simplified system remains maddeningly complex. The benefit of simplifying e-liquid to (PG/GL) is that thermal decomposition data collected by ${ }^{1} \mathrm{H}$ NMR spectroscopy is not littered with the resonance signals of compounds like nicotine, benzaldehyde, vanillin, and other compounds present to varying degrees in commercially available flavored e-liquids. As discussed in Chapter 1, Section 4.5, the ${ }^{1} \mathrm{H}$ NMR spectrum of un-degraded (PG/GL) contains no peaks in the regions typically associated with aldehydes, aromatics, alkenes, and other deshielded protons which may be observed if detectable levels of decomposition products are present. Such peaks are occluded by downfield resonance signals corresponding to nicotine and flavorants - compounds that 
may comprise greater than $1 \%$ of the molecules present in commercial e-liquid products, depending on the potency and flavor.

\subsection{Collecting Samples of Vaporized Electronic Cigarette Liquid for NMR}

\section{Analysis}

Under the slight vacuum of inhalation, e-liquid leaves the mouthpiece of an actuated ecigarette as a mixture of aerosol droplets and vapors. Analyzing the composition of this mixture and understanding how it differs from the e-liquid remaining unvaporized in the reservoir is the central subject of this investigation; as such, a simple method was developed to collect samples of vaporized (PG/GL) immediately after they exit the mouthpiece. This method collects samples into glass vials capped with polytetrafluoroethylene (PTFE) septa. Samples may be collected into empty vials, or into vials containing some specific volume of $\mathrm{DMSO}^{-\mathrm{D}_{6}}$ which has been delivered by pipet. A short length of silicone rubber tubing connects the mouthpiece of an e-cigarette to a disposable needle, which pierces the septum and delivers the vaporized sample into the vial. Sterile single-use disposable needles are ideal to avoid contamination between samples; large inner-diameter needles such as 16 or 18 gauge afford unrestricted air flow through the system. A second disposable needle also pierces the septum and connects the vial to a large plastic syringe via another short length of silicon rubber tubing. This sample collection setup is shown in Figure 3.1 , with a $1.5 \mathrm{~mL}$ glass vial connected to a $50 \mathrm{~mL}$ plastic syringe and an e-cigarette composed of a KangerTech ${ }^{\circledR}$ Protank II cartomizer affixed to an Innokin ${ }^{\circledR}$ iTaste V4 battery. 


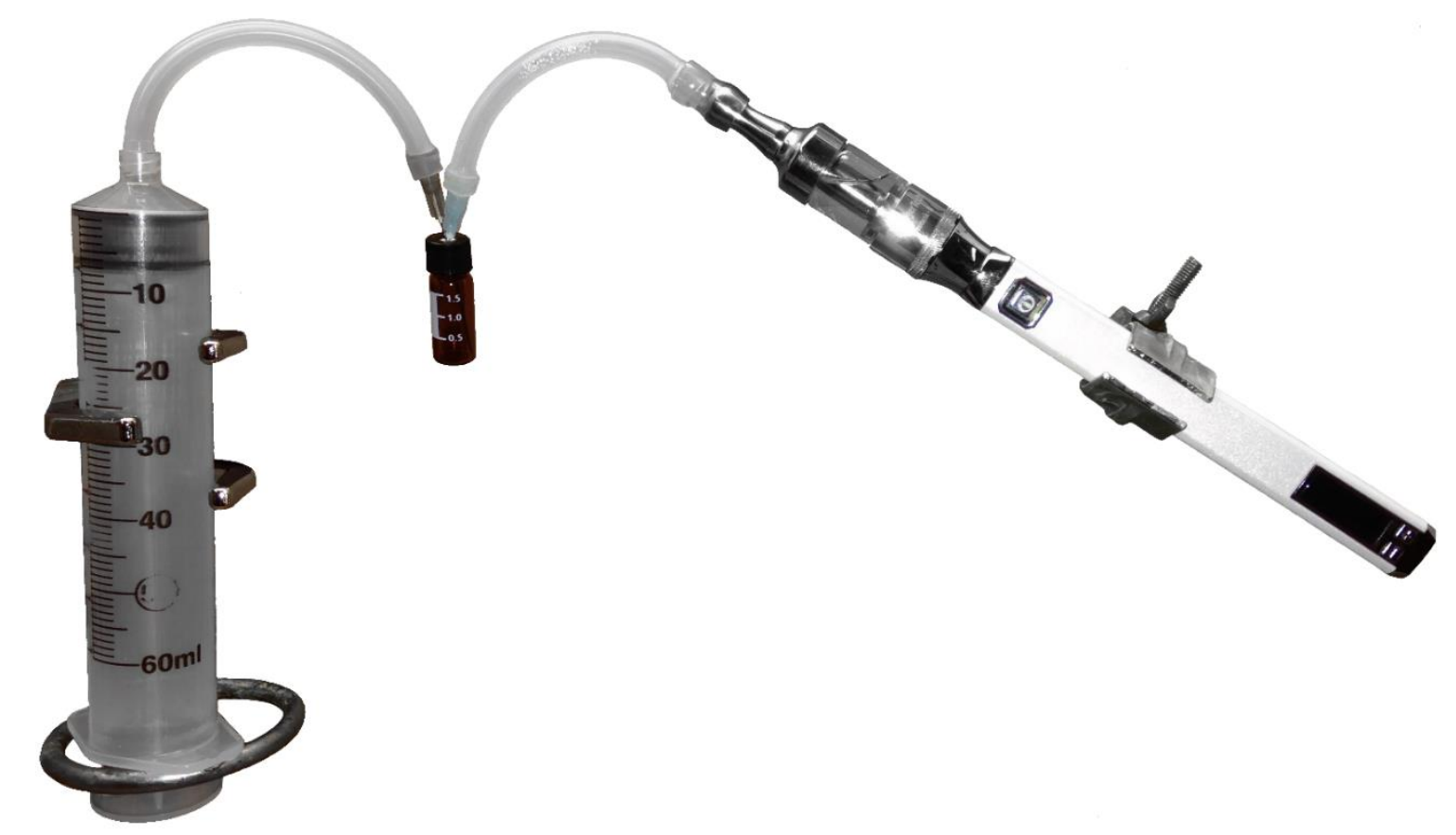

Figure 3.1 - Vaporized Sample Collection Apparatus.

When the syringe plunger is pulled down, the resulting vacuum draws air through the vent holes in the e-cigarette, past the heating coil and out the mouthpiece before entering the glass vial through the incident needle and exiting through the outlet needle towards the emptying barrel of the syringe. If the e-cigarette is actuated during this syringe pull, aerosolized e-liquid will accompany the flowing air from the heating coil through the glass vial and into the syringe; some of the e-liquid aerosols and vapors are drawn into the solvent as they flow through the vial. The mass of the vial and the e-liquid reservoir are recorded before and after sample collection to determine the mass of (PG/GL) consumed and collected. The e-cigarette is actuated with the right hand while the syringe is pulled down with the left. The syringe plunger is pulled smoothly down to the $50 \mathrm{~mL}$ graduation over a desired puff duration such as 3,5 , or 10 seconds. The barrel of the syringe begins filling 
with e-liquid immediately upon actuating and pulling. A metronome has been found a useful aid to keep consistent timing. The method allows a single investigator to collect samples alone without an expensive syringe pump or smoking machine.

Samples collected by this method are consumed from the reservoir at consistent rates, but the mass of vaporized material collected in each sample vial is less consistent. This method of collection is straightforward and effective, but it is not perfect; samples never retain all of the material aerosolized during a puff and might be regarded as an incomplete profile of the vaporized material - that fraction which did not make it all the way to the syringe, being drawn instead into the solvent or onto the inner walls of the glass vial.

\subsection{Electronic Cigarette Liquid Consumption}

E-liquid is consumed from the reservoir of an e-cigarette at a rate that is dependent on the behavior of the user. The temperature of the saturated heating element is a function of a number of variables including the operating power of the e-cigarette, the duration of the puff, and the volume of air pulled past the heating coil during the puff. Holding two of these conditions fixed while varying the third demonstrates a correlation between the variable condition and the amount of e-liquid consumed. Two of these correlations are demonstrated in Figures 3.2 and 3.3. In Figure 3.2, an increase in the mass of (PG/GL) consumed during sample collection is observed as a function of increasing the operating power of the e-cigarette, which was varied in 1 Watt increments. 


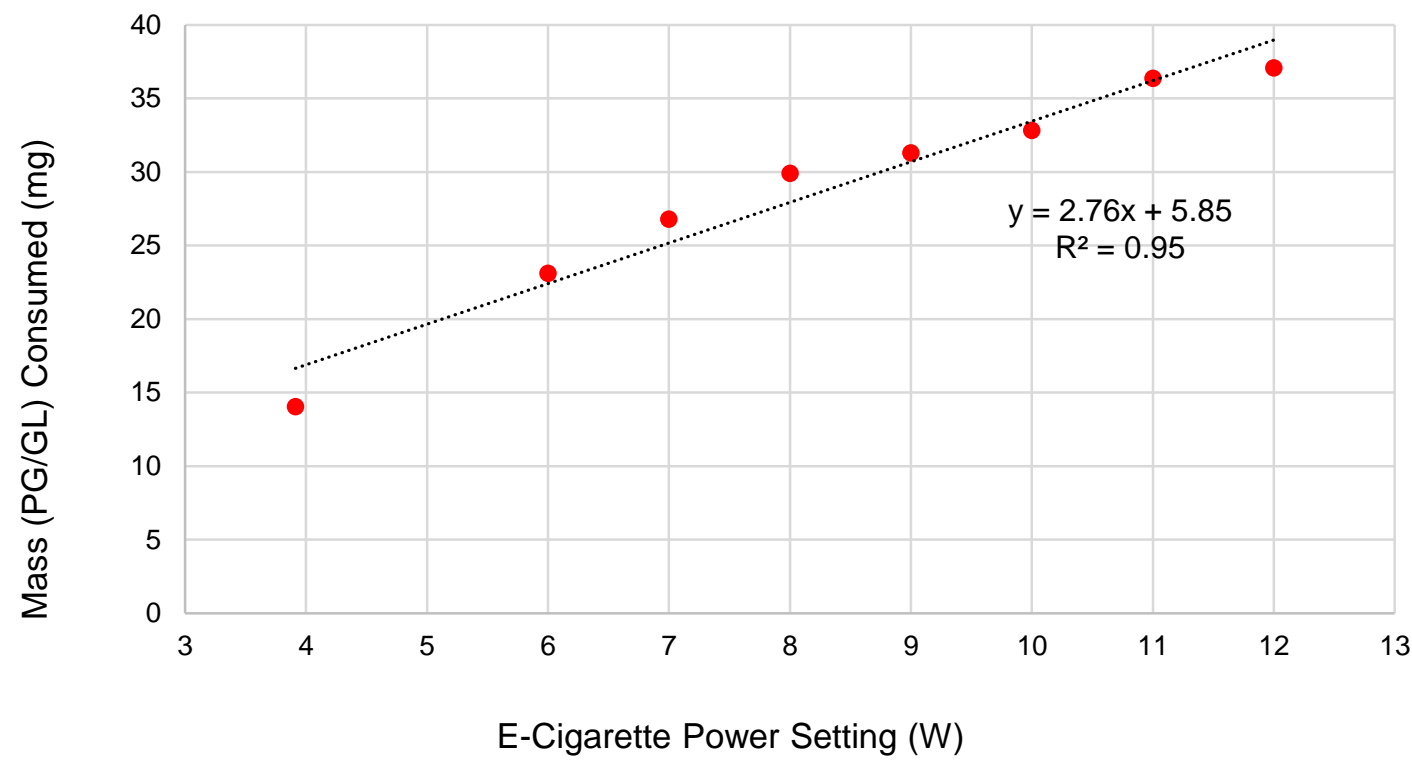

Figure 3.2 - Consumption of (PG/GL) Increases with Increased Operating Power.

The samples represented in the above figure were composed of three $50 \mathrm{~mL}$ puffs vaporized with a 5 second puff duration using an inexpensive CE4 cartomizer affixed to a variablevoltage battery. The observed increase in e-liquid consumption with increasing operating power at a fixed flow rate indicates that the temperature of the heating element increases with increasing operating power.

In Figure 3.3, an increase in the mass of (PG/GL) consumed during sample collection is observed as a function of increased puff duration. The samples summarized in this figure were collected in triplicate and were composed of a single $50 \mathrm{~mL}$ puff vaporized at $12 \mathrm{~W}$ using a KangerTech ${ }^{\circledR}$ Protank II cartomizer and a variable-voltage battery. The observed increase in e-liquid consumption with increasing puff duration at a fixed operating power 
and puff volume indicates that the temperature of the heating element increases with decreasing flow rate.

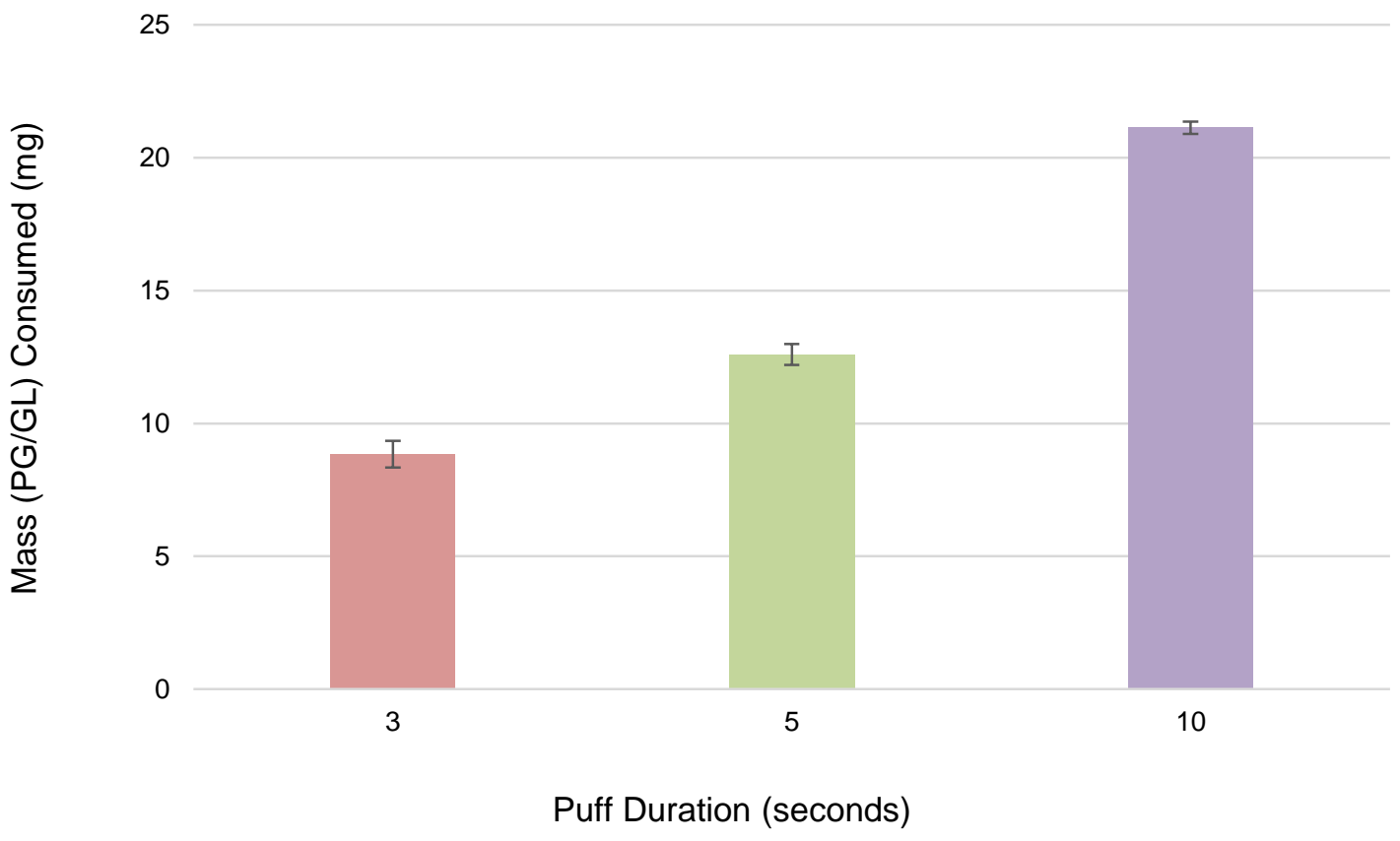

Figure 3.3 - Consumption of (PG/GL) Increases with Increased Puff Duration.

The mass of (PG/GL) consumed from the e-liquid reservoir during sample collection serves as a convenient handle by which to gauge the quality of each vaporized sample, guarding against anomalous results that could arise from an atypical sample. For example, if the wick of the heating element was not completely wetted with e-liquid during sample collection, or if the device were not fully actuated during the puff, then the low mass of (PG/GL) consumed during the collection of that particular sample might provide an indication of abnormality prior to collecting and analyzing decomposition data. The mass 
of (PG/GL) consumed during the collection of each sample also allows for the extrapolation of molecular concentrations quantified within the sample into rates of exposure; by assuming an average e-liquid consumption rate such as $3000 \mathrm{mg} / \mathrm{day}$, the daily exposure of any quantified species can be calculated. This type of treatment is interesting but relies on many assumptions of uniformity.

\subsection{Electronic Cigarette Liquid Degradation}

Glycerol and propylene glycol can both be aerosolized using an e-cigarette without any evidence of degradation by operating the device in a manner intended to minimize the temperature of the heating element. The absence of decomposition products in samples of (PG/GL) vaporized using an e-cigarette set to the lowest power setting serves as a metric of quality assurance for the experimental technique, indicating that contamination is not introduced into samples during collection or their subsequent preparation for analysis. The lowest power setting on the Innokin ${ }^{\circledR}$ iTaste V4 variable-wattage battery used in this investigation is 6 Watts, although an even lower power level of $\sim 4$ Watts is possible using the lowest voltage setting of 3.0 volts while operating in variable-voltage mode with $a \sim 2$ $\Omega$ heating coil. As the operating power of the e-cigarette rises, new magnetic resonance signals unrelated to (PG/GL) begin to appear in the ${ }^{1} \mathrm{H}$ spectra of vaporized (PG/GL) samples. This is demonstrated in Figure 3.4, which shows the ${ }^{1} \mathrm{H}$ NMR spectrum of unvaporized (PG/GL) plotted along with a sample vaporized from a KangerTech ${ }^{\circledR}$ Protank II cartomizer on the lowest voltage setting ( $4 \mathrm{~W})$ and the lowest wattage setting $(6 \mathrm{~W})$. The spectrum of the sample vaporized at 4 Watts contains only a few very small peaks associated with decomposition products. The spectrum of the sample vaporized at 6 Watts 
contains more peaks than the spectrum from the lower power sample, and some of the signals have a much higher intensity.

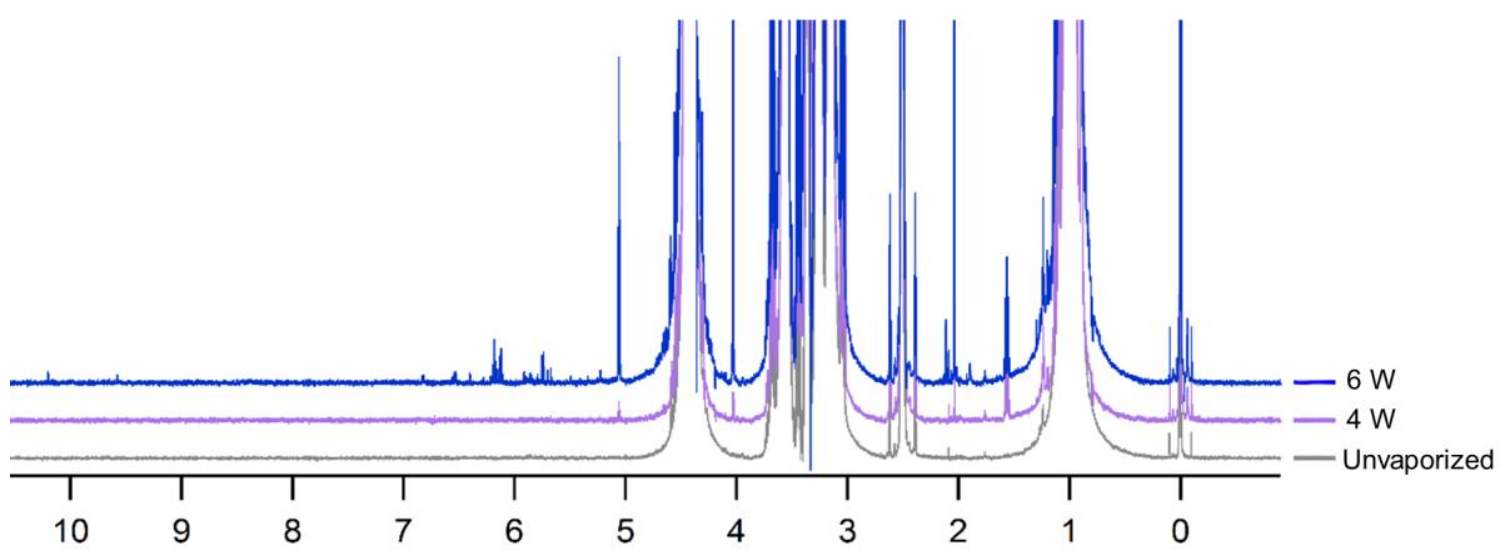

Figure 3.4 $-{ }^{1} \mathrm{H}$ NMR Spectra of (PG/GL) Vaporized Using Low Power Settings.

When using a KangerTech ${ }^{\circledR}$ Protank II cartomizer, decomposition is usually evident in the ${ }^{1} \mathrm{H}$ NMR spectra of single-puff samples of (PG/GL) vaporized around or above 10 Watts, although small peaks often appear at much lower power levels as seen in Figure 3.4.

Spectra collected from samples vaporized at high power levels may contain sizable proportions of over a dozen different decomposition products. As operating power increases, the profile of degradation products found in samples changes; signals corresponding to certain decomposition products continue to increase in intensity, while other signals corresponding to intermediates in decomposition pathways may decrease or disappear altogether. This increase in decomposition product diversity and abundance is demonstrated in Figure 3.5, featuring ${ }^{1} \mathrm{H}$ NMR spectra of (PG/GL) samples vaporized in a 
single $50 \mathrm{~mL}$ puff lasting 5 seconds from a Sailebao ${ }^{\circledR}$ CE4 cartomizer at variable power levels.

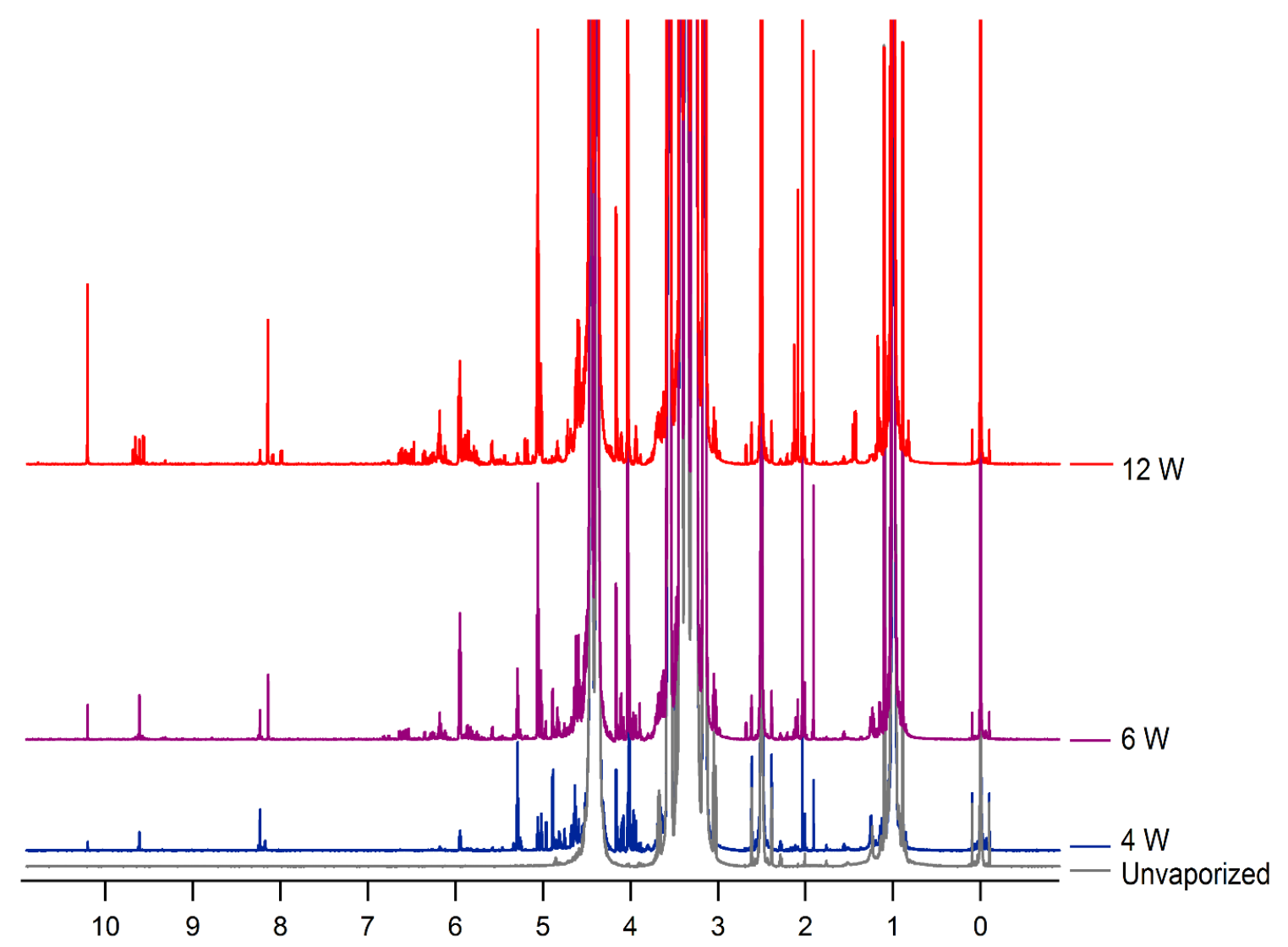

Figure 3.5 - Increased Abundance and Diversity of Decomposition Products with Increased Power.

Samples vaporized at very high temperatures, using the highest possible power setting and the longest possible puff duration of the battery $(15 \mathrm{~W}, 10 \mathrm{sec})$, can contain abundances of diverse and reactive chemical species. The trend of increased decomposition with increased operating power is typified in Figure 3.6, showing a series of ${ }^{1} \mathrm{H}$ NMR spectra collected 
from samples composed of single $(50 \mathrm{~mL}, 5 \mathrm{sec})$ puffs of (PG/GL) vaporized from a Sailebao ${ }^{\circledR}$ CE4 cartomizer at varying wattage levels using an Innokin ${ }^{\circledR}$ iTaste V4 battery.

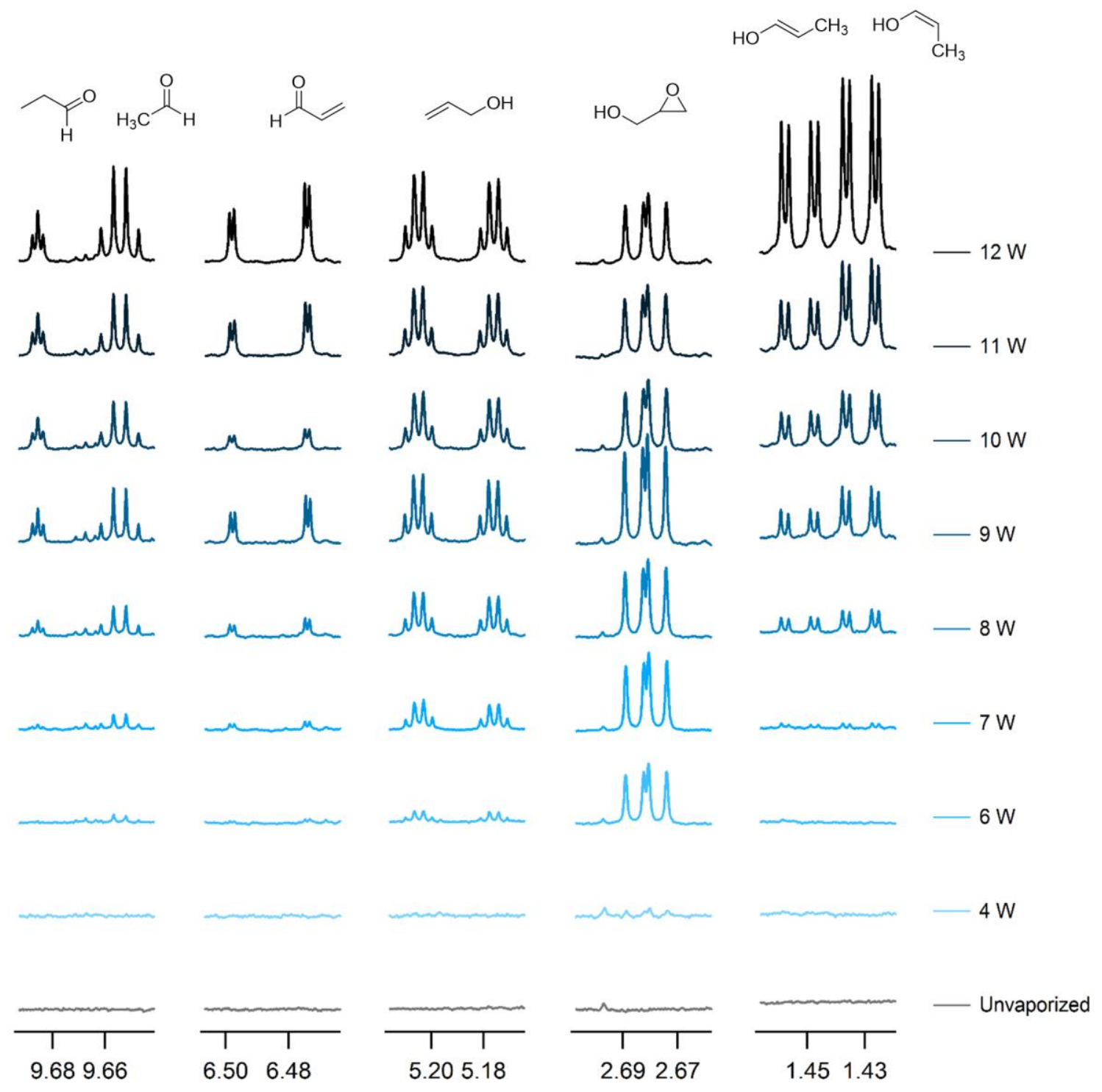

Figure 3.6 - Selected Peaks from the ${ }^{1} \mathrm{H}$ NMR Spectra of (PG/GL) Samples Vaporized with Increasing Power. 


\subsubsection{Identification and Confirmation of Decomposition Products}

Vaporized samples of (PG/GL) were collected directly into aliquots of DMSO-D 6 contained within pre-weighed glass vials capped with PTFE septa; during quantitative experiments, the solvent was carefully prepared with an internal standard $(1,2,4,5-$ tetrachloro-3-nitrobenzene at $1.0 \mathrm{mM}$ ) for quantification by relative ${ }^{1} \mathrm{H}$ integration. After collection, the vials were weighed to determine the mass of samples collected, and the solutions were transferred to $5 \mathrm{~mm}$ glass NMR tubes for analysis. At high magnetic fieldstrengths, one-dimensional ${ }^{1} \mathrm{H}$ analysis is straightforward with as little as one single $50 \mathrm{~mL}$ puff of (PG/GL) consisting of 5-10 mg, although many scans may be required to collect a spectrum with an acceptable signal-to-noise ratio. Samples consisting of three to ten $50 \mathrm{~mL}$ puffs of (PG/GL) significantly cut down on acquisition time and allow for much easier one-dimensional ${ }^{13} \mathrm{C}$, COSY, TOCSY, NOESY, ${ }^{1} \mathrm{H}-{ }^{13} \mathrm{C}$ HSQC and HMBC experiments. Collecting too many puffs of vaporized (PG/GL) may result in overly-concentrated samples and diminished quality of observed ${ }^{1} \mathrm{H}$ signals; an abundance of glycerol makes samples viscous and increases the rate of exchange, diminishing peaks and collapsing the observed splitting of exchangeable protons such as hydroxyls. When studying glycerol by ${ }^{1} \mathrm{H}$ NMR, increased signal intensity is bought at the expense of fine detail.

After a compound has been speculatively identified by chemical shift, peak shape, coupling constants, and relative integration, the identity of the product is confirmed by adding a very small portion of the pure compound obtained from a commercial source to the sample and 
observing a small increase in the intensity of the peaks under investigation. If sufficiently little of the pure compound is added, the results of such an experiment can provide confirmation of identity; if too much is added, the addition of large resonances to the spectrum will overwhelm the peaks under investigation and no confirmation can be made. The data generated by this minute addition experiment is demonstrated in Figure 3.7, showing a successful confirmation of acetone in a sample of vaporized (PG/GL). In green, plotted across the top, is the ${ }^{1} \mathrm{H}$ NMR spectrum of pure acetone diluted in DMSO- ${ }_{6}$. In grey, plotted across the bottom, is the ${ }^{1} \mathrm{H}$ NMR spectrum of unvaporized (PG/GL), which contains no peaks in the acetyl region. In blue is plotted the ${ }^{1} \mathrm{H}$ NMR spectrum of a sample of vaporized (PG/GL) containing new peaks in the acetyl region corresponding to decomposition products. When a very small amount of acetone is added to the sample of vaporized (PG/GL), one of the peaks in the acetyl region grows, while the others remain at the same intensity, as shown in the spectrum plotted in the red dotted line. Figures such as these are presented for a number of decomposition products in this chapter. 

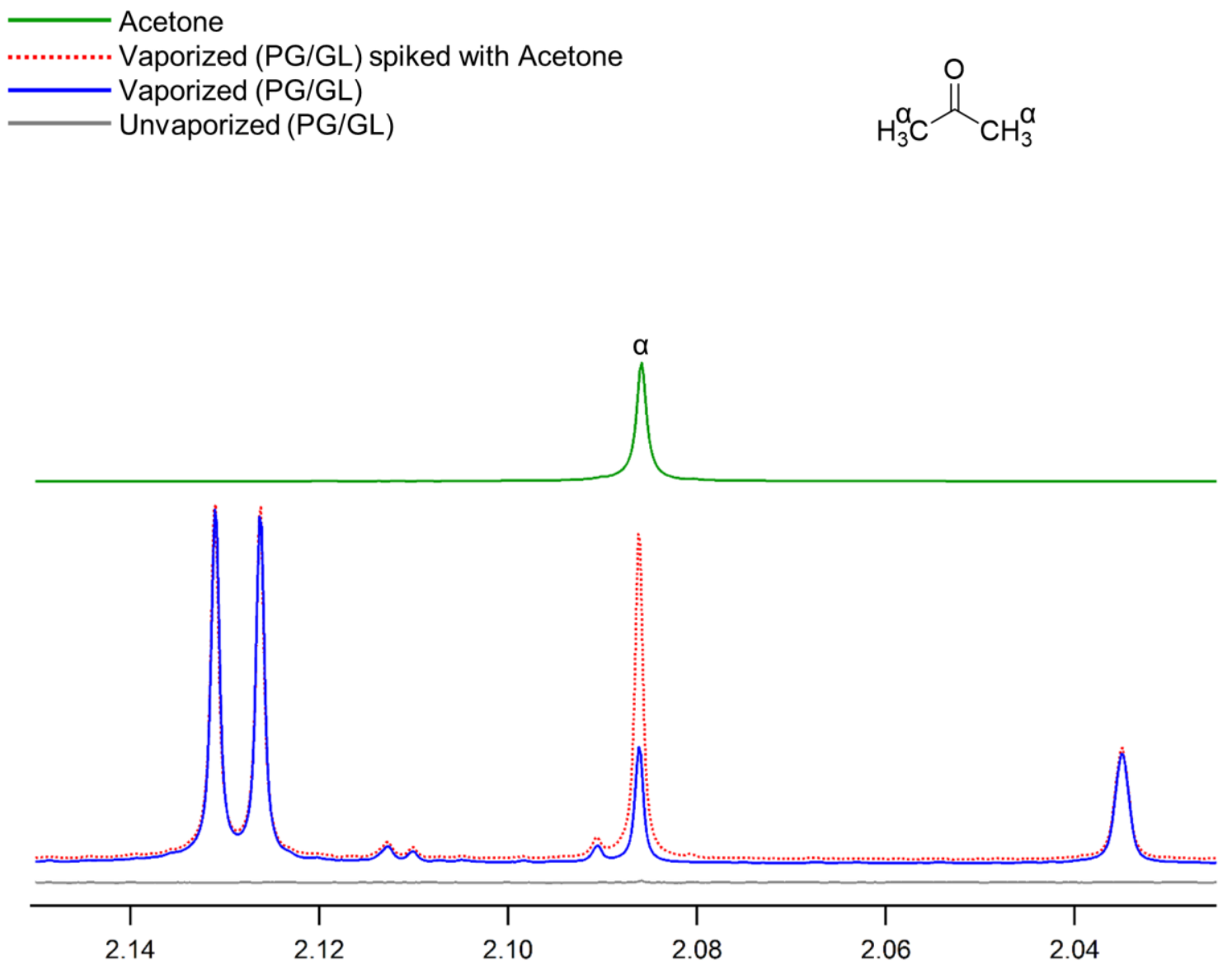

Figure 3.7 - Confirmation of Decomposition Product Identity by Minute Addition.

\subsubsection{Glycerol Degradation}

\subsubsection{Glycerol Dehydration}

Glycerol has been understood to dehydrate into acrolein since 1843, when Josef Redtenbacher reported his isolation of the $\alpha, \beta$-unsaturated aldehyde by distillation of glycerol in the presence of dehydrating agents. ${ }^{36}$ John Nef explained in 1904 that formation of this double-dehydration product occurs in two steps: initially, water is lost 
from the $\beta$-carbon atom, forming an unstable intermediate that Nef called a dioxypropylidene which undergoes intramolecular rearrangement into 3hydroxypropanal, a $\beta$-hydroxyaldehyde that eliminates a second equivalent of water and forms acrolein in a dehydration reaction analogous to the final step of an aldol condensation. ${ }^{44}$ This double-dehydration is summarized in Scheme 3.1.

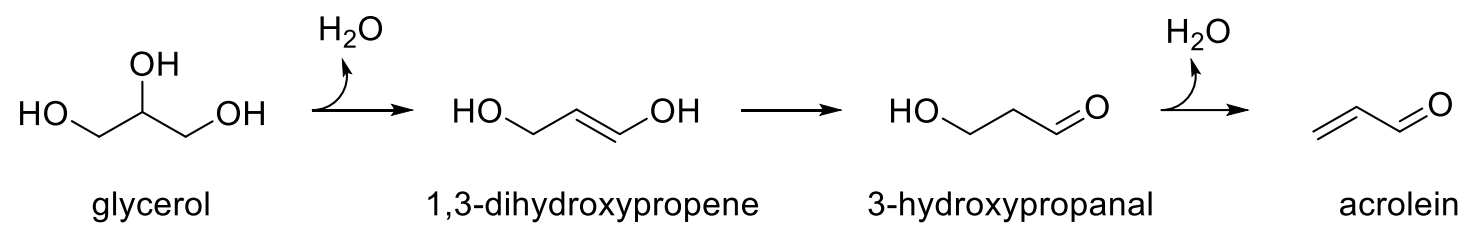

Scheme 3.1 - Double Dehydration of Glycerol into Acrolein Proposed by Nef.

In his discussion of the pathway depicted in Scheme 3.1, Nef contends that glycidol is a potential product of the dehydration of glycerol but would be further degraded under his experimental conditions. He proposed that at lower temperatures the dioxypropylidene might transform into glycidol, which he and others had previously produced by heating glycerol to $240{ }^{\circ} \mathrm{C}$ in the presence of acetic acid or glyceric acid. At $450{ }^{\circ} \mathrm{C}$, the temperature of his decomposition experiments, Nef knew that glycidol rearranged preferentially into hydroxyacetone, forming only trace amounts of acrolein; hydroxyacetone went on to dissociate into formaldehyde and acetaldehyde. This more complex discussion of the dehydration of glycerol is summarized in Scheme 3.2. 


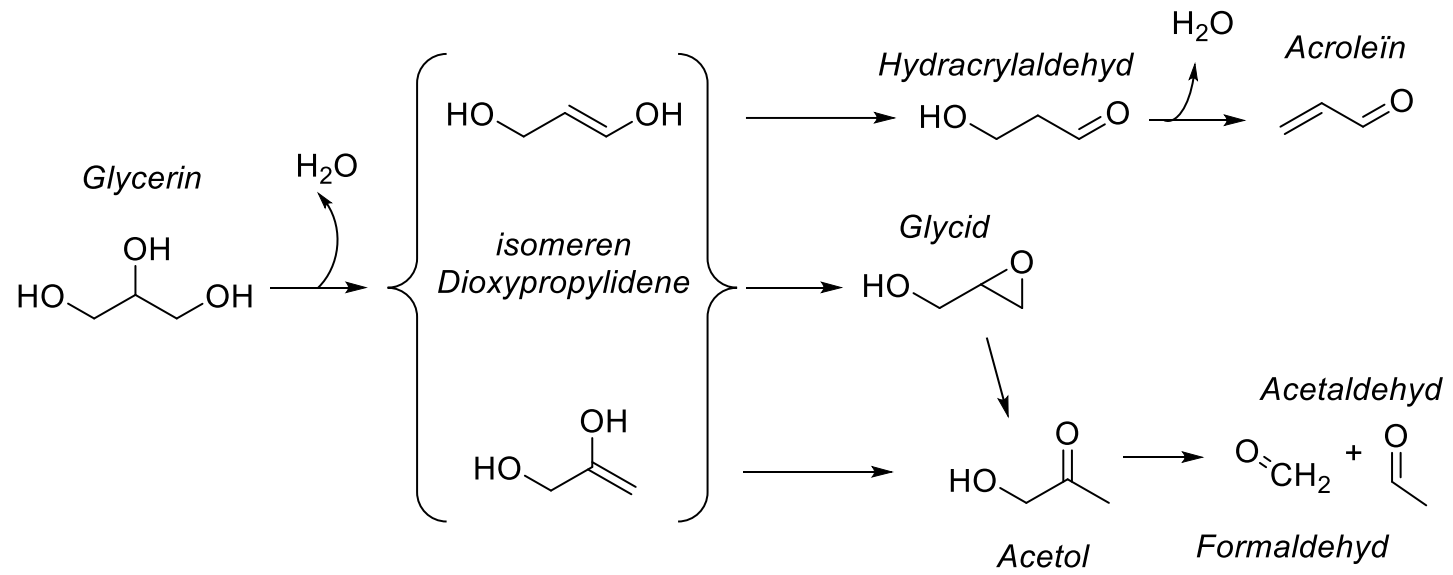

Scheme 3.2 - Dehydration and Subsequent Degradation of Glycerol as Discussed by Nef.

Thus, glycerol forms three primary dehydration products; 3-hydroxypropanal, glycidol, and hydroxyacetone. Each of these primary dehydration products is sufficiently reactive to further decompose under the conditions investigated by Nef. The pathways described in Scheme 3.2 are remarkably similar to the pathways proposed in a 2011 article published in The Journal of Physical Chemistry A titled "A Revisited Picture of the Mechanism of Glycerol Dehydration" by authors Laino et al,${ }^{75}$ who seem to have been unfamiliar with Nef's discussion of glycidol.

\subsection{Hydroxyacetone}

Hydroxyacetone is often the most populous decomposition product present in samples of vaporized (PG/GL), being a primary dehydration product of glycerol and a primary oxidation product of propylene glycol (see Section 3.4.3.2). When samples are vaporized at low temperatures by using low power settings and short puff durations, this compound is sometimes the only degradation product detected. This is typified in Figure 3.4, which 
shows a ${ }^{1} \mathrm{H}$ NMR spectrum of (PG/GL) vaporized at 4 Watts containing little evidence of decomposition products save a few small peaks corresponding to hydroxyacetone. As the power level and/or puff duration used to collect vaporized (PG/GL) samples increases, hydroxyacetone continues to increase in intensity; only rarely is it outnumbered by any other single decomposition product. The structure of hydroxyacetone is shown in Figure 3.8.

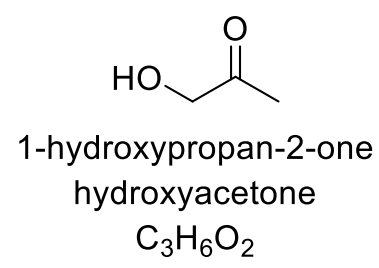

Figure 3.8 - Molecular Structure of Hydroxyacetone.

The compound has a relatively simple structure to solve and confirm using ${ }^{1} \mathrm{H}$ and ${ }^{13} \mathrm{C}$ NMR data. Having a ketone (and thus no protons) at the $\beta$-carbon, the resonance signal of the adjacent acetyl group (s, $2.04 \mathrm{ppm}$ ) remains a Lorentzian singlet located near the signals of other acetyl compounds such as acetone and acetic acid. The two equivalent methylene protons (d, $4.02 \mathrm{ppm})$ are split into a doublet by the adjacent hydroxyl proton (t, $5.06 \mathrm{ppm})$, which is likewise split into a triplet by the methylene protons. The chemical shift of the methylene doublet falls between the large resonances of the hydroxyl and alkyl protons of un-degraded (PG/GL) and is un-occluded in samples of vaporized (PG/GL) when observed at high frequency. The hydroxyl proton is labile to hydrogen-deuterium exchange experiments, disappearing from the spectrum while the methylene doublet collapses into a singlet. The chemical shift of the hydroxyl proton is very susceptible to changes in 
temperature or concentration. The acetyl resonance, being un-split and composed of three equivalent protons, is much taller than the other signals. The assigned ${ }^{1} \mathrm{H}$ NMR spectrum of hydroxyacetone is shown in Figure 3.9, along with confirmation of the compound in vaporized (PG/GL) by minute addition.
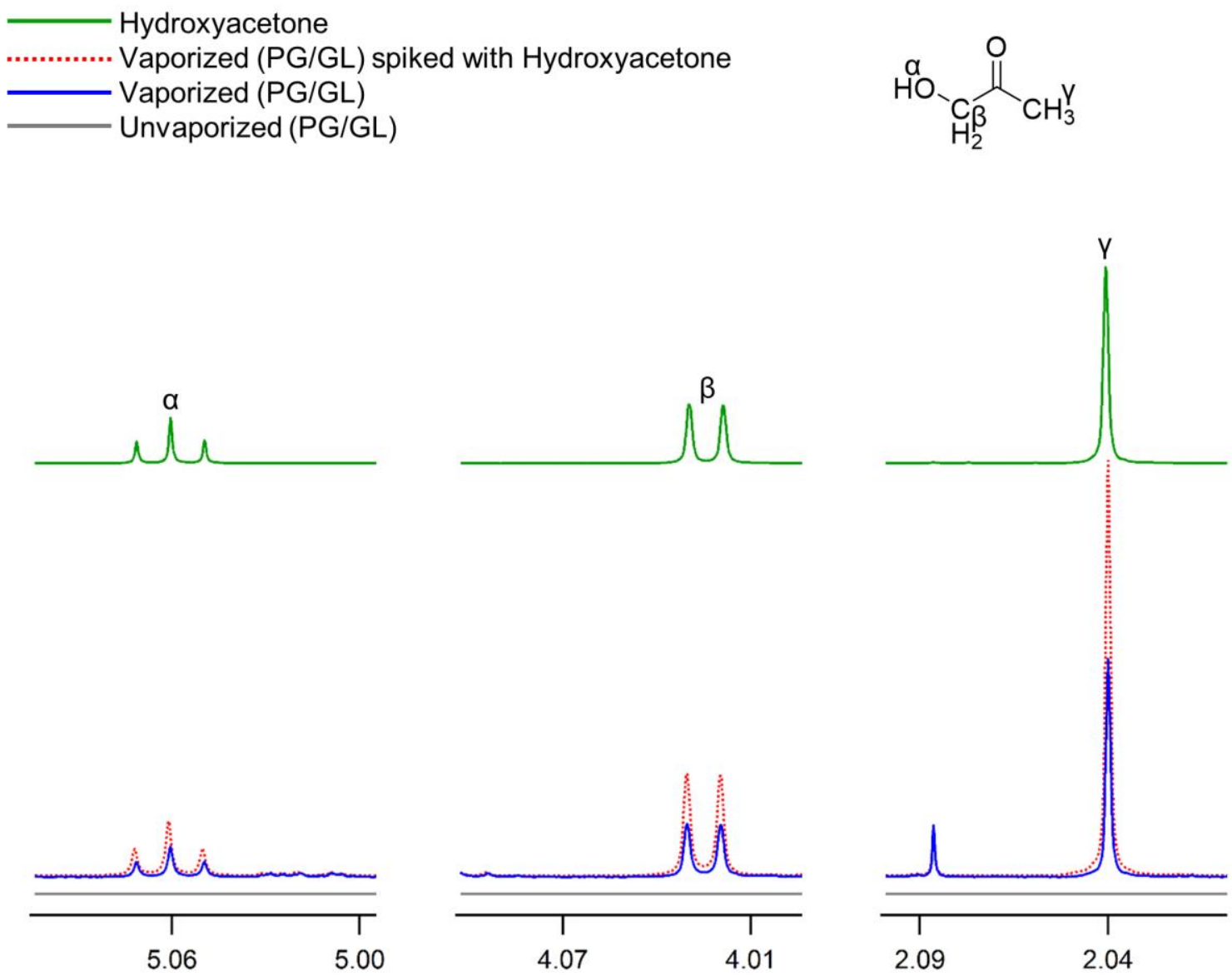

Figure 3.9 $-{ }^{1} \mathrm{H}$ NMR Assignment of Hydroxyacetone and Confirmation in Vaporized (PG/GL). 
Note that in the vaporized (PG/GL) spectrum, the small singlet peak corresponding to acetone (s, $2.08 \mathrm{ppm})$ does not change in intensity upon minute addition of hydroxyacetone to the vaporized (PG/GL) sample.

\subsection{Acrolein}

The simplest $\alpha, \beta$-unsaturated aldehyde, acrolein is a double-dehydration product of glycerol which grows steadily in abundance as e-cigarette operating power is increased. The structure of acrolein is shown in Figure 3.10.

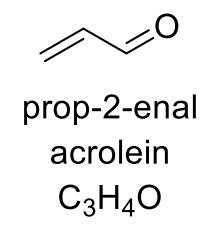

Figure 3.10 - Molecular Structure of Acrolein.

The compound has distinct ${ }^{1} \mathrm{H}$ resonances that are easily recognized but unfortunately overlap with a number of compounds found in vaporized (PG/GL) samples. Having one proton in the $\alpha$-position (the carbon bound to the carbonyl), the aldehyde resonance (d, $9.57 \mathrm{ppm})$ is split into a wide doublet. This $\alpha$-proton (ddd, $6.34 \mathrm{ppm})$ is split into a complex resonance (a doublet-of-doublets-of-doublets) by the aldehyde and the two non-equivalent geminal protons on the adjacent $\beta$-carbon, which can themselves be assigned by their vicinal cis (dd, $6.62 \mathrm{ppm})$ and trans $(\mathrm{dd}, 6.48)$ coupling with the $\alpha$-proton. The aldehyde proton is sometimes partially occluded by a sharp singlet corresponding to monomeric formaldehyde. One of the geminal protons is usually un-occluded in the downfield region. Acrolein is often among the most abundant degradation species present in vaporized 
samples of (PG/GL) collected at high temperatures by using high power levels and long, slow puffs. The assigned ${ }^{1} \mathrm{H}$ NMR spectrum of acrolein is shown in Figure 3.11, along with confirmation of the compound in vaporized (PG/GL) by minute addition.
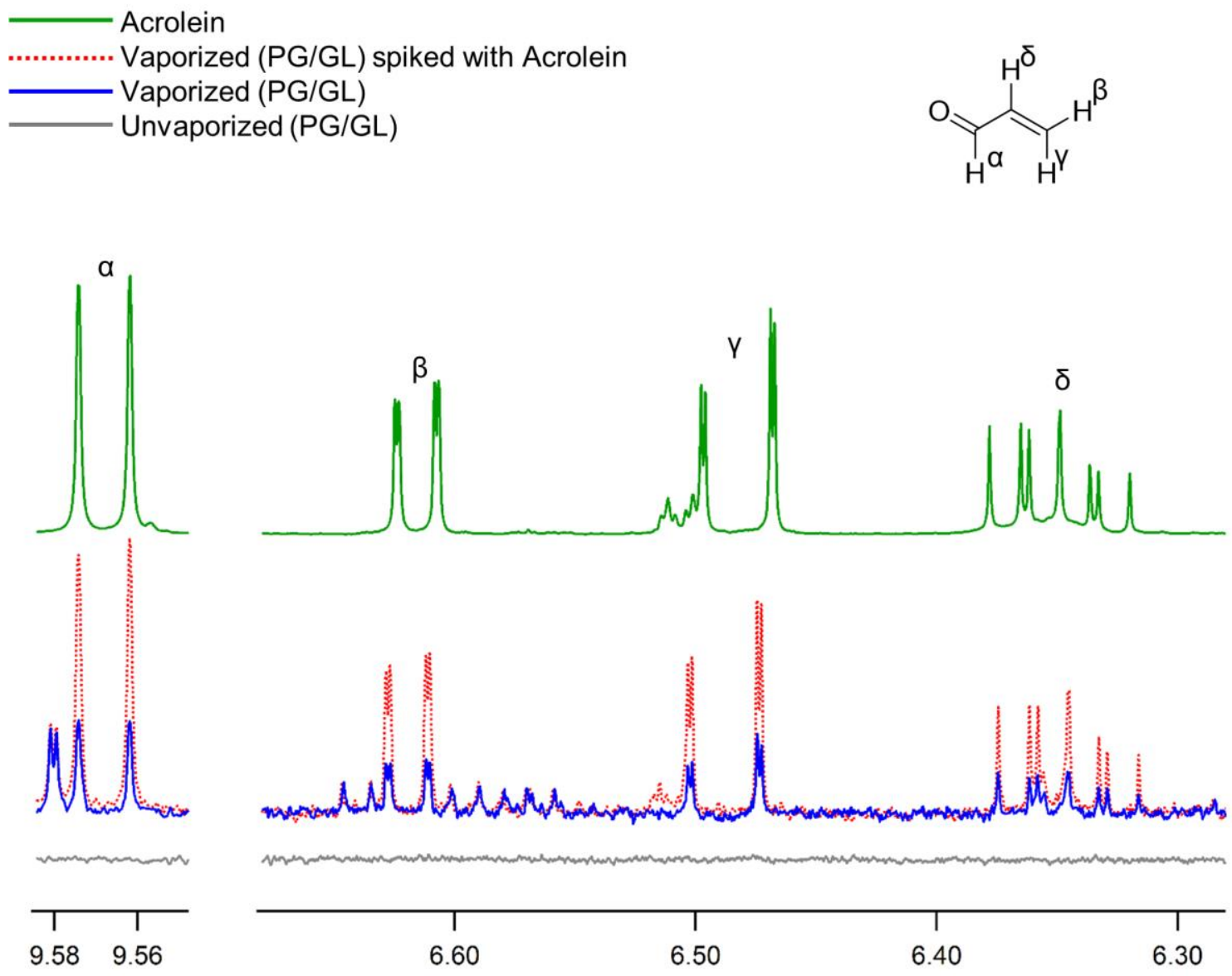

Figure 3.11 - ${ }^{1} \mathrm{H}$ NMR Assignment of Acrolein and Confirmation in Vaporized (PG/GL).

\subsection{Glycidol}

Glycidol is a chiral oxirane compound with an unusual ${ }^{1} \mathrm{H}$ NMR spectrum. The structure of glycidol is shown in Figure 3.12. 


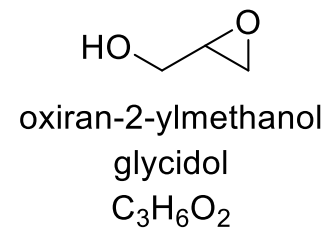

Figure 3.12 - Molecular Structure of Glycidol.

The extreme bond angles of the epoxide ring give atypical geometry to the ring-bound protons, which are non-equivalent. Two resonances corresponding to ring protons appear between the residual DMSO peak and the (PG/GL) methylene protons, the more upfield a doublet-of-doublets (dd, $2.66 \mathrm{ppm}$ ), and the more downfield a complex crown-shaped set of peaks (m, $2.98 \mathrm{ppm}$ ). At $400 \mathrm{MHz}$, these signals are unresolved from and occluded by the ${ }^{13} \mathrm{C}$ satellite peaks of the much larger resonances of DMSO and (PG/GL). A triplet corresponding to the hydroxyl proton (t, $4.84 \mathrm{ppm})$ is sometimes visible downfield of the (PG/GL) hydroxyls. The partially assigned ${ }^{1} \mathrm{H}$ NMR spectrum of glycidol is shown in Figure 3.13, along with confirmation of the compound in vaporized (PG/GL) by minute addition. 

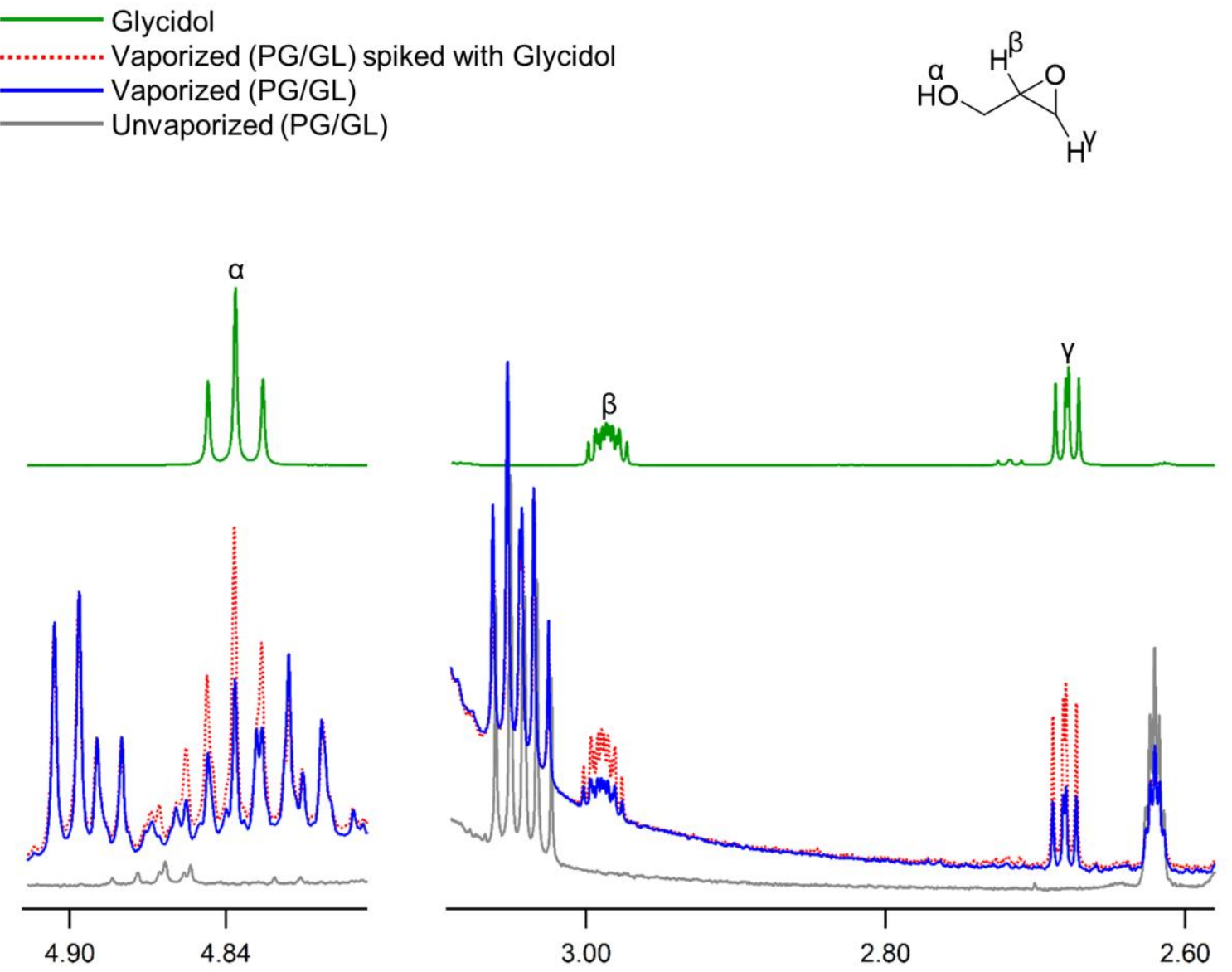

Figure 3.13 $-{ }^{1} \mathrm{H}$ NMR Assignment of Glycidol and Confirmation in Vaporized (PG/GL).

Glycidol has previously been described as a dehydration product of glycerol by Nef, Laino et al., and Nimlos et al., ${ }^{44,75,99}$ but at the time of this writing, I do not believe it has been reported as a product of vaporized e-liquid. This compound never grows to a very high abundance, possibly reflecting the reactivity shown in Scheme 3.2.

\subsection{Acetaldehyde}

The structure of acetaldehyde is shown in Figure 3.14. 


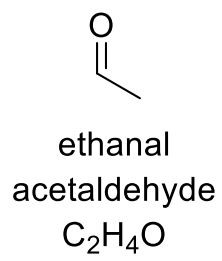

Figure 3.14 - Molecular Structure of Acetaldehyde.

Acetaldehyde is easy to recognize by ${ }^{1} \mathrm{H}$ NMR spectroscopy - the aldehyde resonance is split into an unmistakable quartet (q, $9.65 \mathrm{ppm}$ ) by the three adjacent acetyl protons, while the acetyl resonance $(\mathrm{d}, 2.12 \mathrm{ppm})$ is split by only the single aldehyde proton into a doublet. The assigned ${ }^{1} \mathrm{H}$ NMR spectrum of acetaldehyde is shown in Figure 3.15, along with confirmation of the compound in vaporized (PG/GL) by minute addition. These resonances have a strong correlation by ${ }^{1} \mathrm{H}_{-}{ }^{1} \mathrm{H} \mathrm{COSY}$, and their attachments may be observed by ${ }^{1} \mathrm{H}-$ ${ }^{13} \mathrm{C}$ HSQC and HMBC experiments. This two-carbon compound is a secondary degradation product that grows to very high abundances in samples of (PG/GL) vaporized at high power levels. 

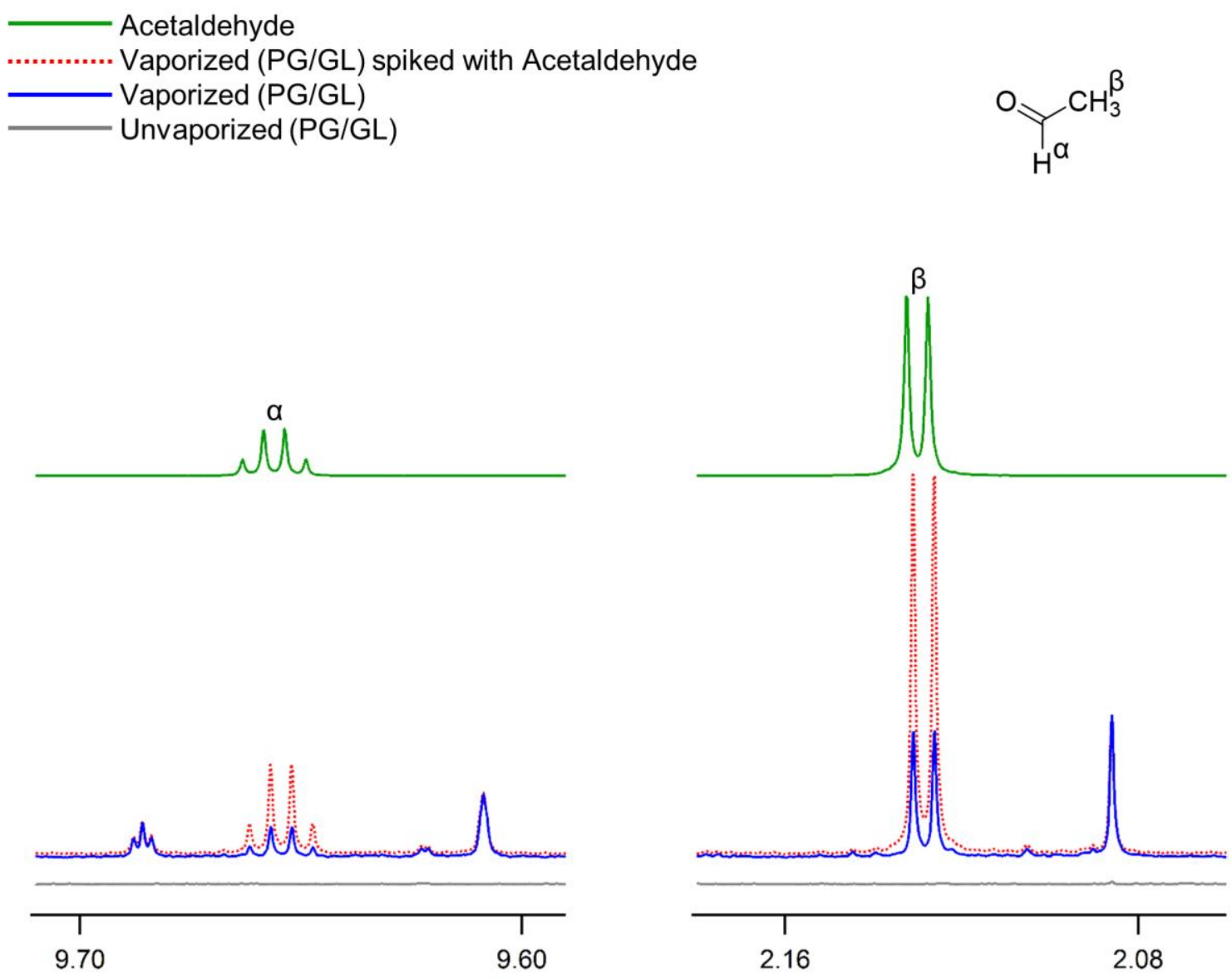

Figure 3.15 $-{ }^{1} \mathrm{H}$ NMR Assignment of Acetaldehyde and Confirmation in Vaporized (PG/GL).

\subsection{Formaldehyde}

Formaldehyde is a volatile and reactive molecule that is not usually encountered in its monomeric form. The sample collection technique described in Section 3.2 does allow for the observation of a sharp singlet peak in the aldehyde region that corresponds to the minor peak present in the ${ }^{1} \mathrm{H}$ spectrum of formalin solution diluted in DMSO-D 6 . In a ${ }^{1} \mathrm{H}$ spectrum collected from a sample of vaporized (PG/GL), this sharp aldehyde resonance overlaps partially with the aldehyde resonance of acrolein before diminishing in intensity over the 
course of several hours and ultimately disappearing entirely from view. This disappearance is coincident with the growth of peaks corresponding to hemiformals of glycerol and propylene glycol, indicating that the carbonyl formaldehyde present has formed hemiacetals with the propanols it encountered while in solution. Formaldehyde is the subject of Chapter 4, which is intended to provide helpful context for understanding the bizarre chemistry in which it participates.

\subsubsection{Glycerol Oxidation}

The oxidation of glycerol in air may be initiated by insertion of $\mathrm{O}_{2}$ into the $\mathrm{C}-\mathrm{H}$ bond of the $\alpha$ - or $\beta$-carbon. The resulting hydroperoxyl compound may decompose to form a hydroperoxyl radical and an alkyl radical, or alternatively a hydroxyl radical and an oxy-

alkyl radical. By abstraction of an additional hydrogen atom, such as the $\mathrm{C}-\mathrm{H}$ of an unreacted equivalent of glycerol, hydroxyl radical forms water and hydroperoxyl radical forms hydrogen peroxide, each leaving behind another alkyl radical, as depicted in Scheme 3.3, which is an original composition that borrows from Diaz et al. and Comprehensive Chemical Kinetics Vol. 17: Gas Phase Combustion., ${ }^{477}$ 


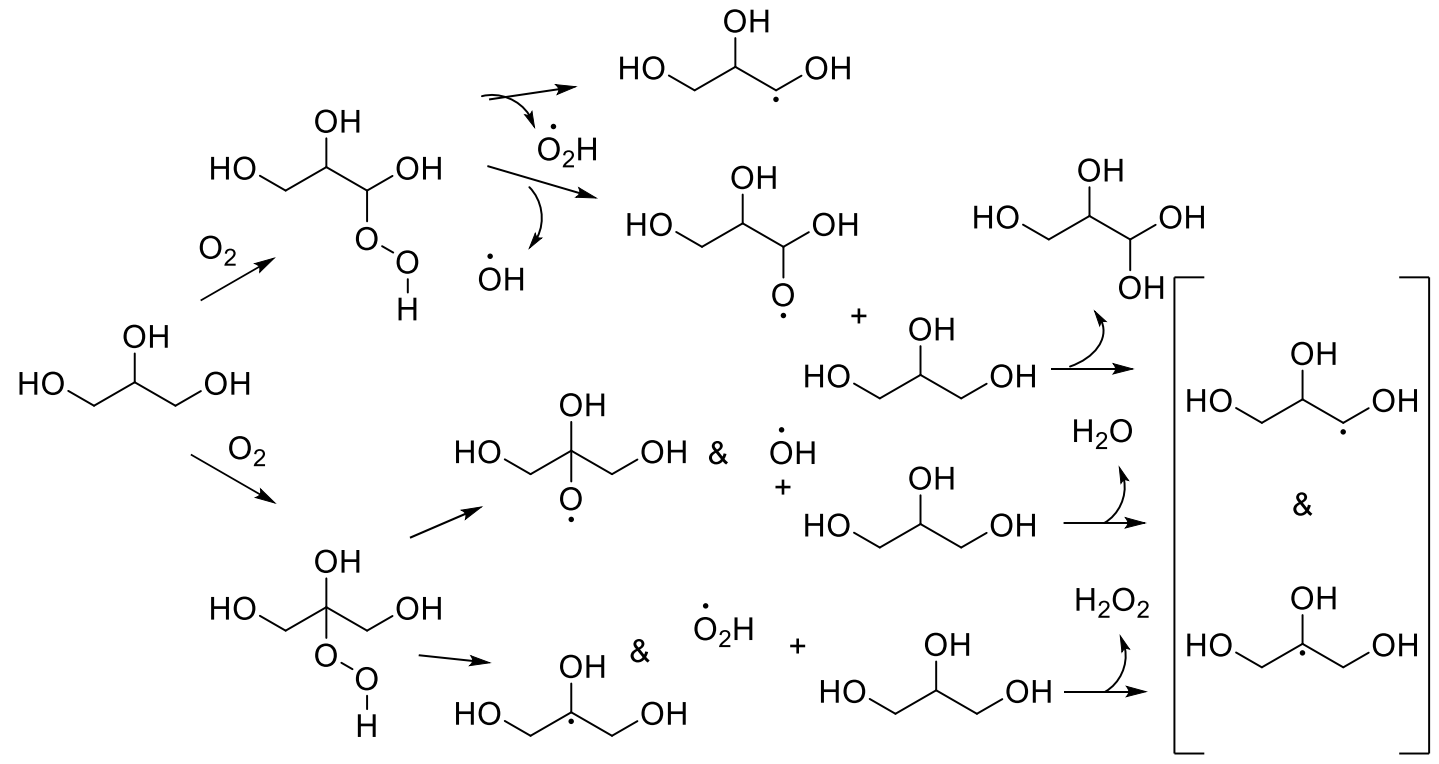

Scheme 3.3 - Initiation of Glycerol Oxidation.

Scheme 3.4 shows the insertion of $\mathrm{O}_{2}$ into the $\mathrm{C}-\mathrm{H}$ bond of the $\alpha$-, $\beta$-carbons of glycerol to form hydroperoxyl compounds, which lose hydroxyl radical and form oxy-alkyl radicals. These $\alpha$-, $\beta$-oxy-alkyl radicals might abstract a hydrogen and form a tetraol, or cleave bonds and form a diverse array of more stable decomposition products while further propagating an equally diverse array of radicals. 


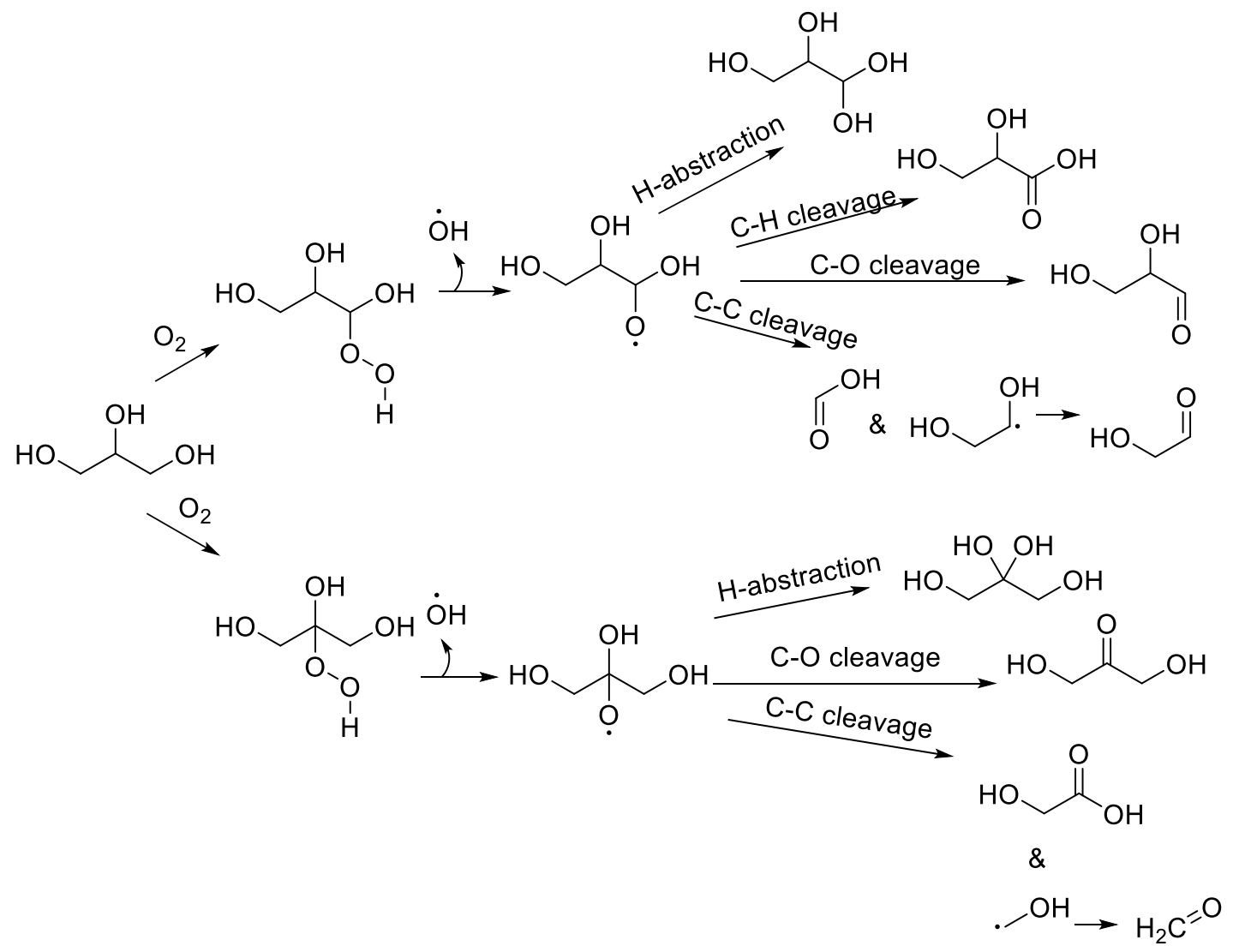

Scheme 3.4 - Glycerol Oxidation - Oxy-alkyl Radicals.

Hydroxy-alkyl radicals of glycerol may decompose by elimination of one of the $\mathrm{C}-\mathrm{O}$ or $\mathrm{C}$ $\mathrm{H}$ bonds. Elimination of one of the $\mathrm{C}-\mathrm{O}$ bonds forms the same isomeric dioxypropylidenes discussed by John Nef in his 1904 publication in Justus Liebig's Annalen der Chemie. ${ }^{44}$ As Nef described, these enols tautomerize to the corresponding ketone or aldehyde product hydroxyacetone and 3-hydroxypropanal - which can further decompose into acrolein, formaldehyde, and acetaldehyde. One might recognize these compounds as the canonical "pyrolysis" products of glycerol, but an oxygen-dependent free radical mechanism provides a competing route to these same products if the system is aerobic; this is not 
typically discussed in the literature. Elimination of one of the $\mathrm{C}-\mathrm{H}$ bonds results in an intermediate which, according to Nef's terminology, might be considered a trioxypropylidene; this di-enol compound undergoes tautomerization to form dihydroxyacetone and glyceraldehyde, which are simple oxidation products of glycerol (meaning that glycerol has lost $\mathrm{H}_{2}$ during the reaction). The elimination, tautomerization, and secondary decomposition reactions discussed above are summarized in Scheme 3.5.

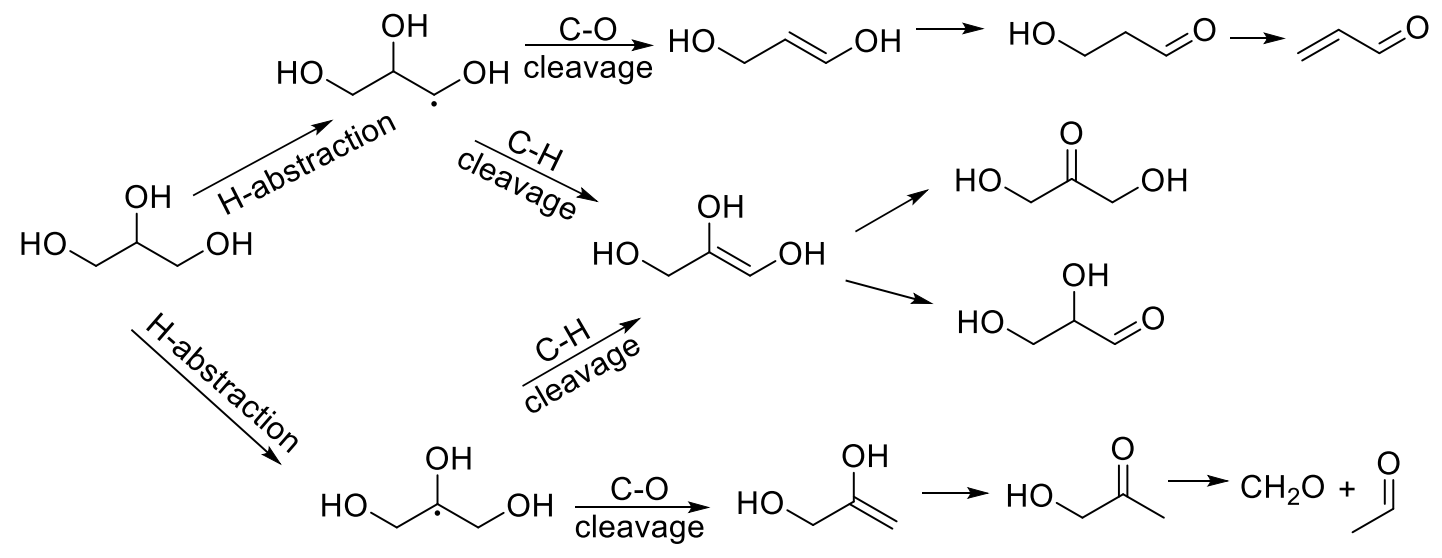

Scheme 3.5 - Glycerol Oxidation - Hydroxyl-alkyl Radicals.

\subsection{Dihydroxyacetone}

The structure of dihydroxyacetone is shown in Figure 3.16.<smiles>O=C(CO)CO</smiles>

\section{1,3-dihydroxypropan-2-one dihydroxyacetone

$$
\mathrm{C}_{3} \mathrm{H}_{6} \mathrm{O}_{3}
$$

Figure 3.16 - Molecular Structure of Dihydroxyacetone. 
Dihydroxyacetone is a symmetrical molecule with an extremely simple ${ }^{1} \mathrm{H}$ NMR spectrum, making it difficult to assign certainly without confirmation by spiking. The signals are very similar to those of hydroxyacetone, but for the absence of an acetyl singlet. One can use ${ }^{1} \mathrm{H}-{ }^{13} \mathrm{C}$ Heteronuclear Multiple Bond Correlation (HMBC) experiments to correlate the methylene protons (, $4.16 \mathrm{ppm})$ with a carbon in the ketone region of the ${ }^{13} \mathrm{C}$ spectrum. The hydroxyl triplet (t, $5.02 \mathrm{ppm}$ ) sits immediately upfield from the nearly identical hydroxyl triplet of hydroxyacetone and is sometimes occluded by ${ }^{1} \mathrm{H}$ resonances from allyl alcohol. The pure form of dihydroxyacetone is often encountered as a dimer and must be dissociated with elevated temperature in dilute solution before it can be used to confirm the presence of the monomer by minute addition. The assigned ${ }^{1} \mathrm{H}$ NMR spectrum of dihydroxyacetone is shown in Figure 3.17, along with confirmation of the compound in vaporized (PG/GL) by minute addition. 

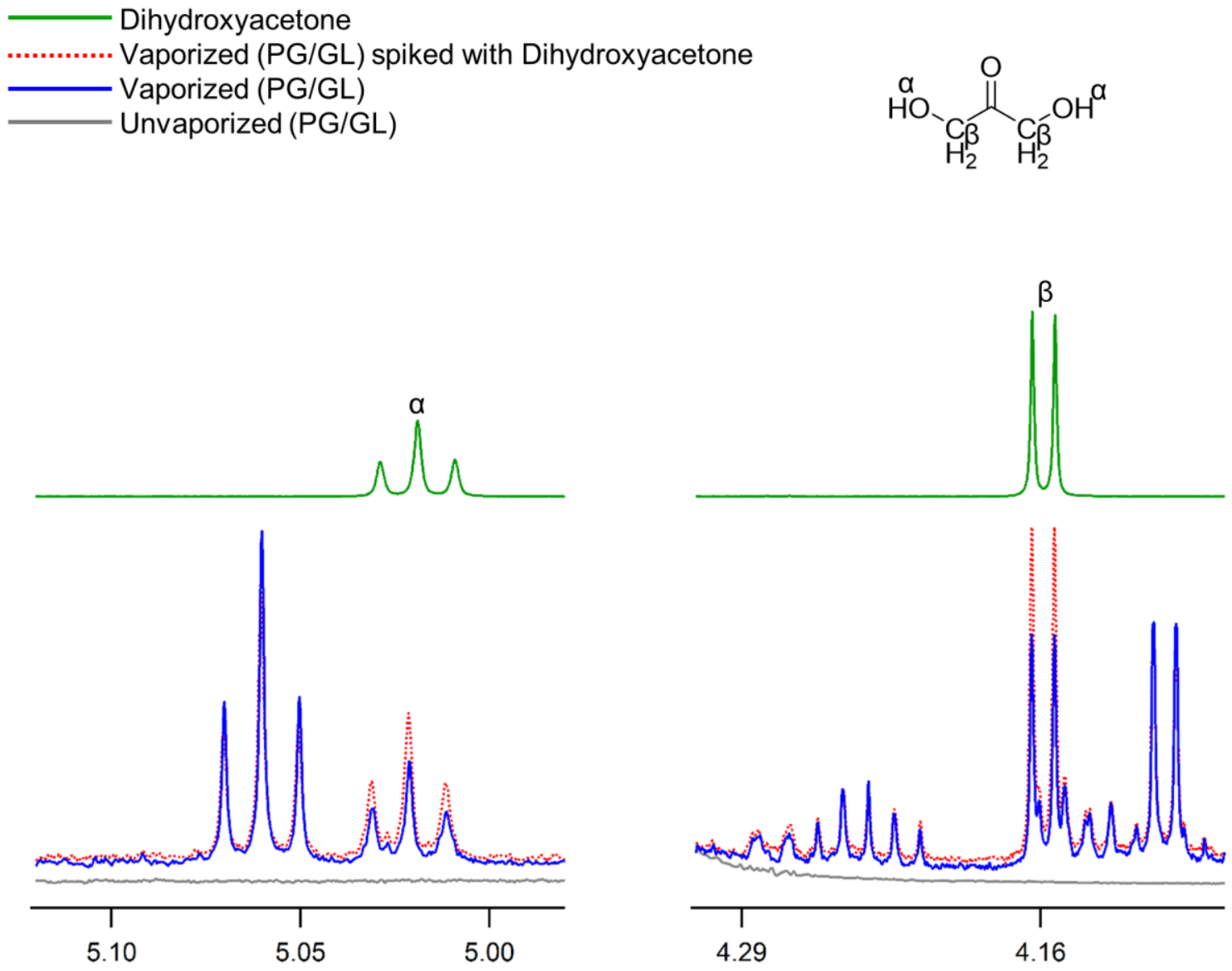

Figure 3.17 - ${ }^{1} \mathrm{H}$ NMR Assignment of Dihydroxyacetone and Confirmation in Vaporized (PG/GL).

\subsection{Glyceraldehyde}

The structure of glyceraldehyde is shown in Figure 3.18.

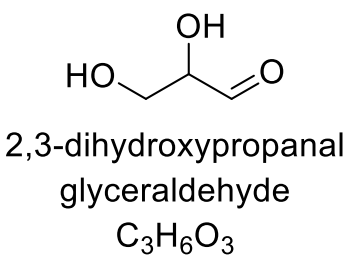

Figure 3.18 - Molecular Structure of Glyceraldehyde. 
Glyceraldehyde is an $\alpha, \beta$-dihydroxyaldehyde that was evoked in the mechanism proposed by Paul Sabatier in the 1918 publication of Comptes Rendus where he described the low temperature oxidation of glycerol by copper and nickel. ${ }^{49}$ This compound is also purchased as a solid dimer that can be dissociated with elevated temperature and will persist as a monomer in solution if sufficiently dilute. The aldehyde resonance of glyceraldehyde $(\mathrm{d}$, $9.62 \mathrm{ppm})$ is a very tightly coupled doublet - a trait that I have found typical of $\alpha$ hydroxyaldehyhdes. Perhaps unsurprisingly, most of the non-aldehyde protons present in glyceraldehyde resonate at frequencies very similar to (PG/GL) and are not well resolved. Glyceraldehyde is never among the most abundant decomposition products of vaporized glycerol and is possibly susceptible to dissociation by a retro-aldol mechanism. The partially assigned ${ }^{1} \mathrm{H}$ NMR spectrum of glyceraldehyde is shown in Figure 3.19, along with confirmation of the compound in vaporized (PG/GL) by minute addition. 

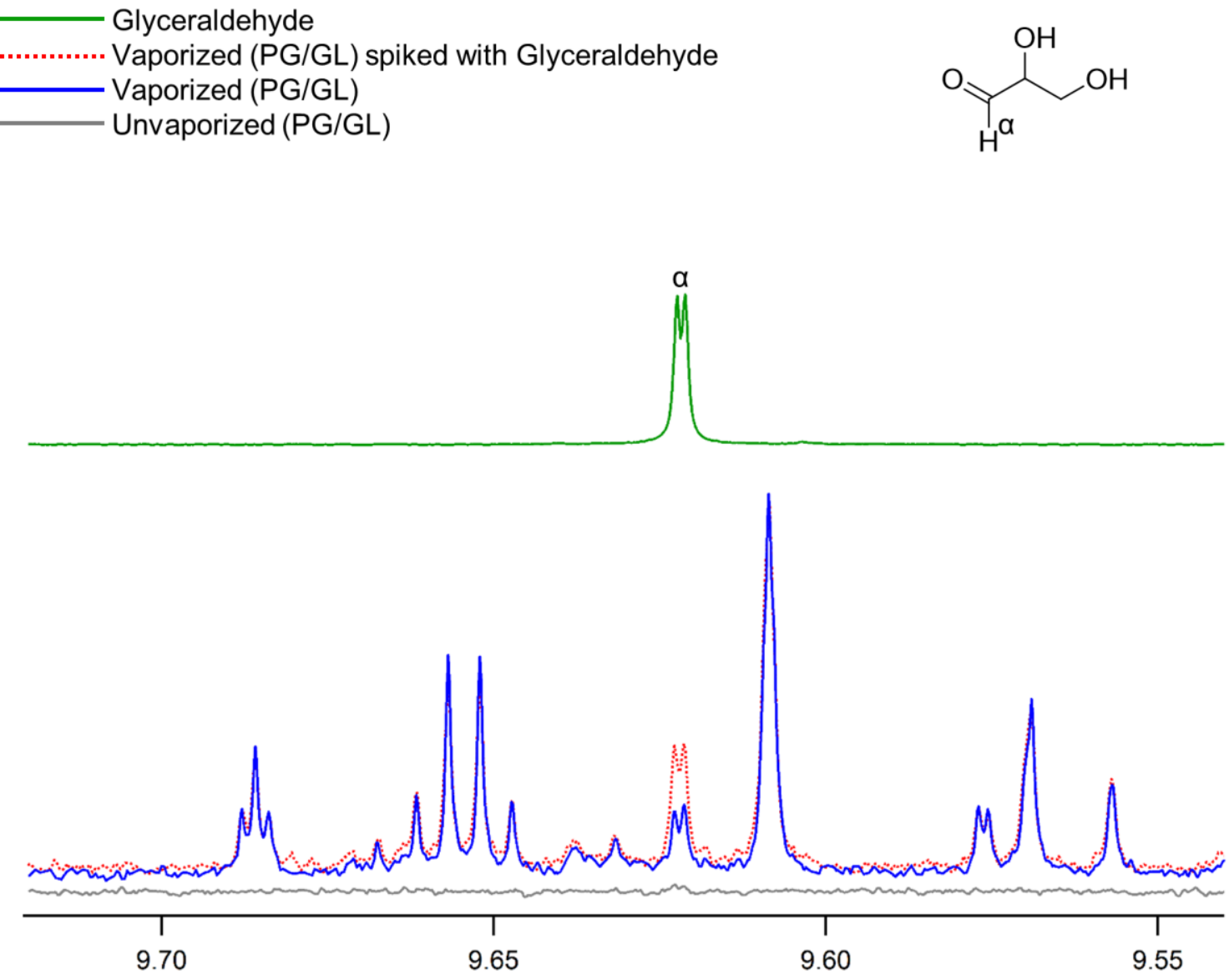

Figure 3.19 - ${ }^{1} \mathrm{H}$ NMR Assignment of Glyceraldehyde and Confirmation in Vaporized (PG/GL).

\subsubsection{Further Glycerol Dissociation}

Many of the primary decomposition products of glycerol, such as hydroxyacetone or 3hydroxypropanal, are susceptible to further decomposition by anoxic dissociation or by CC elimination of oxy-alkyl radicals as discussed above, resulting in one- and two-carbon compounds. These compounds include acetaldehyde and formaldehyde, which have already been discussed, along with a number of other small decomposition products discussed below. 


\subsection{Glycolaldehyde}

Glycolaldehyde is the simplest $\alpha$-hydroxyaldehyde and has a very simple ${ }^{1} \mathrm{H}$ NMR spectrum. One might properly refer to glycolaldehyde as the simplest sugar molecule. This compound is often present in samples of (PG/GL) that have been vaporized under lowtemperature conditions - it is the earliest of the two-carbon species to arise, sometimes present in samples lacking any detectable acetaldehyde or acetic acid. The structure of glycolaldehyde is shown in Figure 3.20.

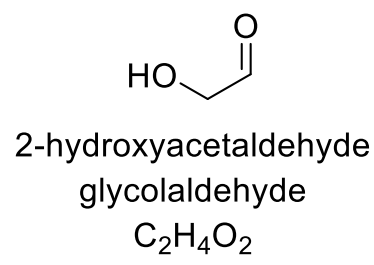

Figure 3.20 - Molecular Structure of Glycolaldehyde.

Like glyceraldehyde and lactaldehyde, which are also $\alpha$-hydroxyaldehydes, the aldehyde resonance of glycolaldehyde is very tightly coupled to the adjacent protons. The aldehyde resonance (t, $9.61 \mathrm{ppm})$ appears to be a singlet to a first approximation, but has triplet character upon close inspection. The methylene protons $(\mathrm{d}, 4.10 \mathrm{ppm})$ are split into a doublet by the hydroxyl proton (t, $5.30 \mathrm{ppm}$ ).

Like dihydroxyacetone and glyceraldehyde, the pure form of this compound is usually encountered as a solid dimer that can be dissociated with heat. The fact that this compound is an intermediate between formaldehyde and glyceraldehyde in the formose reaction (discussed in detail in Chapter 4, Section 5) makes retro-aldol dissociation - similar to the 
dissociation of hydroxyacetone into acetaldehyde and formaldehyde described by Nef - a possibility for the mechanism of glycolaldehyde formation. The assigned ${ }^{1} \mathrm{H}$ NMR spectrum of glycolaldehyde is shown in Figure 3.21, along with confirmation of the compound in vaporized (PG/GL) by minute addition.
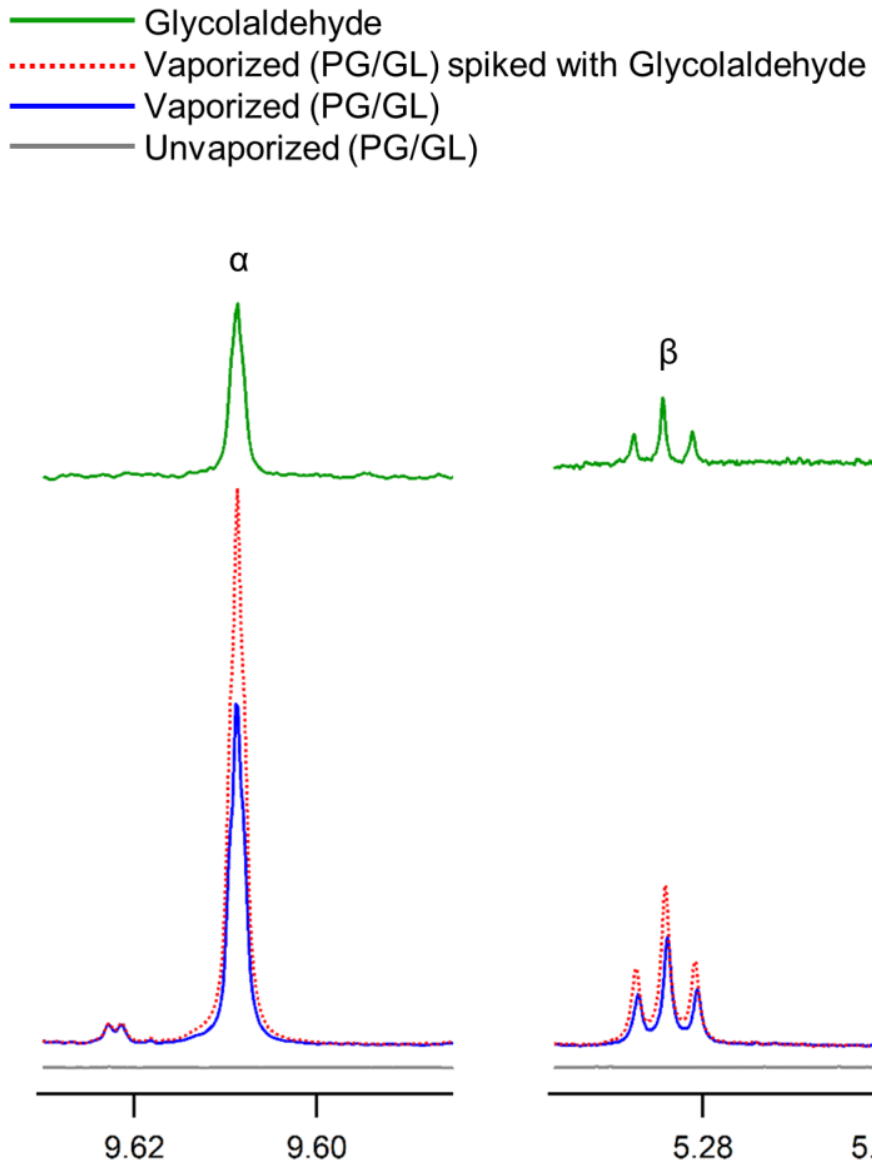
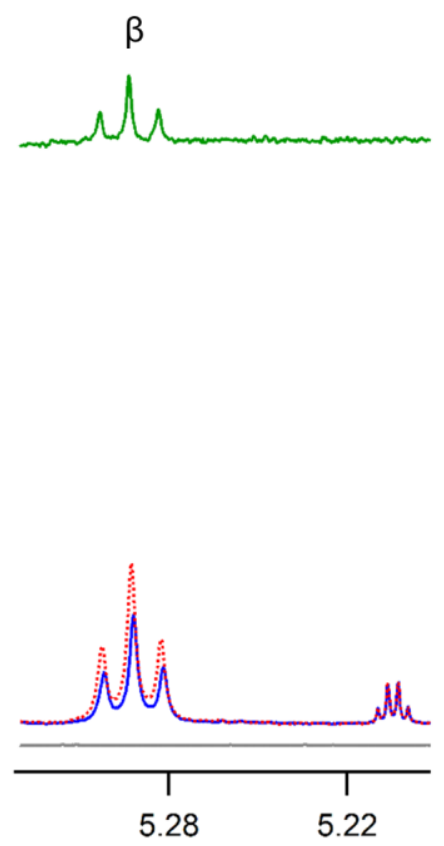
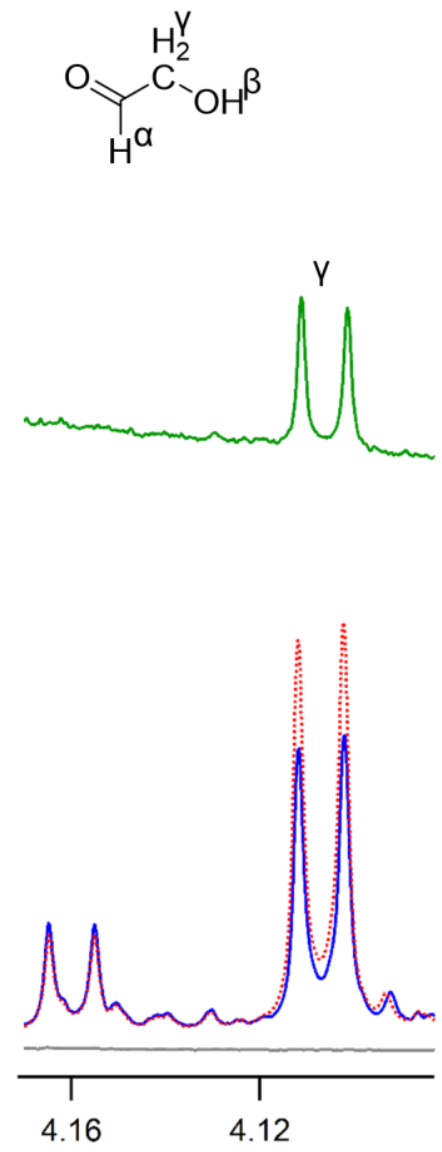

Figure 3.21 $-{ }^{1} \mathrm{H}$ NMR Assignment of Glycolaldehyde and Confirmation in Vaporized (PG/GL).

To determine whether glycolaldehyde might result from glyceraldehyde decomposition, a sample of glyceraldehyde was dissolved to approximately $10 \%$ by mole in propylene glycol. Glycolaldehyde does not form as a result of pure propylene glycol decomposition, 
but was observed as a product in this glyceraldehyde-doped sample, making glyceraldehyde a possible intermediate between glycerol and glycolaldehyde.

\subsection{Allyl alcohol}

Allyl alcohol is both a decomposition product of glycerol and a dehydration product of propylene glycol. The structure of allyl alcohol is shown in Figure 3.22.

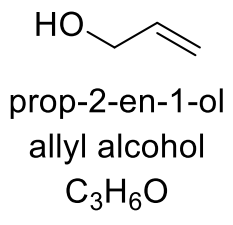

Figure 3.22 - Molecular Structure of Allyl Alcohol.

The geminal protons (trans: dq, $5.19 \mathrm{ppm}$; cis: dq, $5.02 \mathrm{ppm}$ ) of the alkene appear distinctly as doublets-of-quartets, being split by one another, the vicinal proton, and the nearby methylene protons. The lone proton (tt, $5.91 \mathrm{ppm})$ on the middle carbon appears as a triplet-of-triplets, being split by the adjacent methylene protons and by the two terminal alkene protons. The methylene protons (m, $3.93 \mathrm{ppm})$ are split by many protons and appear as a complex crown-shaped resonance. The hydroxyl proton ( $\mathrm{t}, 4.71 \mathrm{ppm})$ is a sharp triplet, being split by the two adjacent methylene protons. The assigned ${ }^{1} \mathrm{H}$ NMR spectrum of allyl alcohol is shown in Figure 3.23, along with confirmation of the compound in vaporized (PG/GL) by minute addition. 

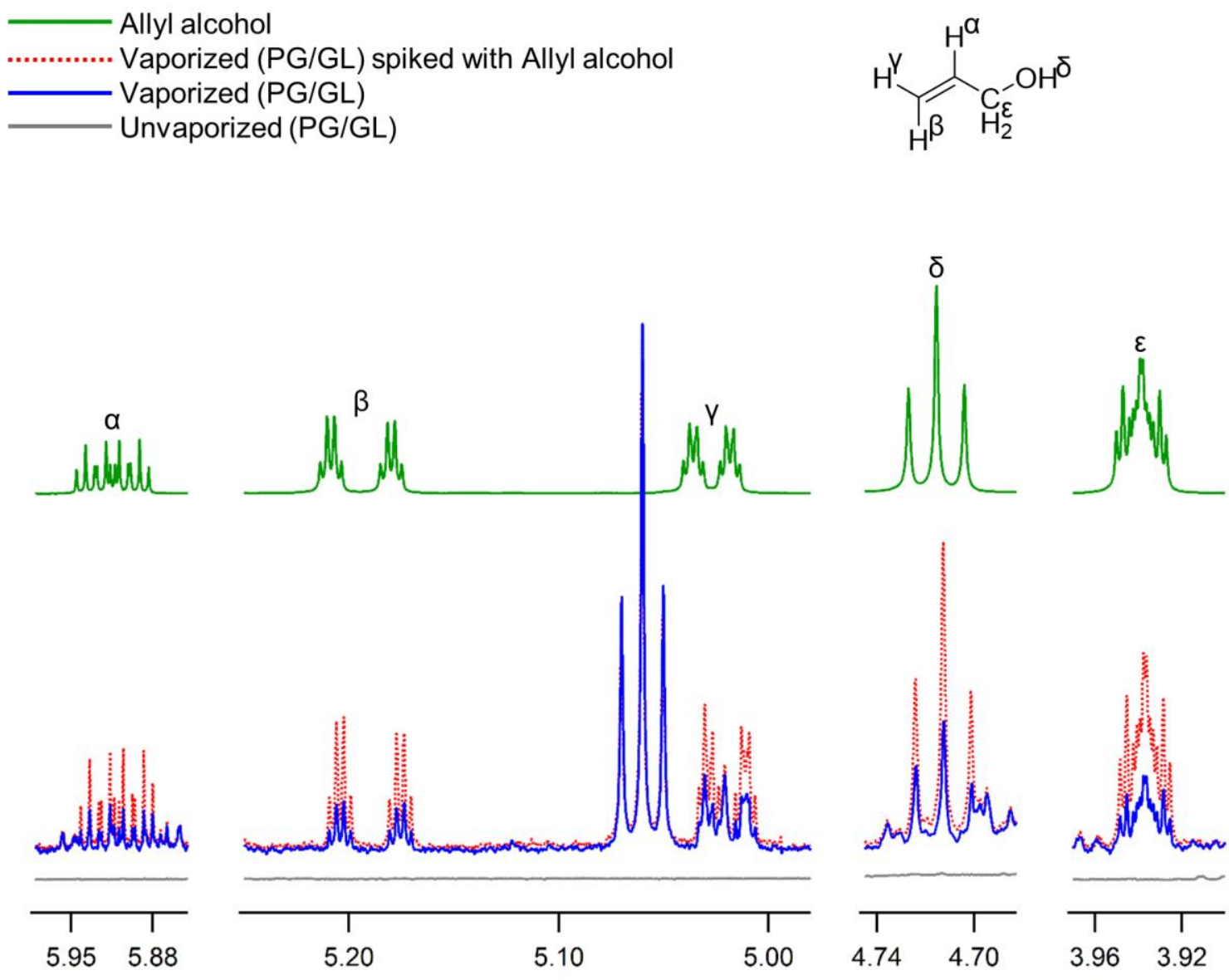

Figure 3.23 - ${ }^{1} \mathrm{H}$ NMR Assignment of Allyl Alcohol and Confirmation in Vaporized (PG/GL)

The mechanism of allyl alcohol formation from glycerol is not straightforward; neither a simple dehydration nor a simple oxidation, glycerol must lose $\mathrm{H}_{2} \mathrm{O}_{2}$ in order to become allyl alcohol. This transformation may occur in several steps and could be mediated by reaction with formic acid, as has been reported previously. ${ }^{100}$ 


\subsection{Acetic Acid}

Acetic acid was reported as a decomposition product of glycerol by Pelouze in his 1836 article Memoire sur la Glycérine published in Annales de Chimie et de Physique. ${ }^{31}$ The structure of acetic acid is shown in Figure 3.24.

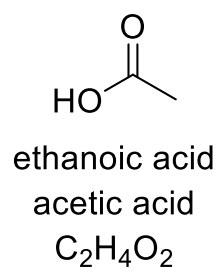

Figure 3.24 - Molecular Structure of Acetic Acid.

Acetic acid is a decomposition product of both glycerol and propylene glycol. The ${ }^{1} \mathrm{H}$ NMR spectrum of acetic acid contains a sharp singlet (s, 1.91 ppm) in the acetyl region and a broad singlet in the carboxylic acid region - the acid peak is not particularly useful, being broad due to rapid exchange and erratic in chemical shift. The partially assigned ${ }^{1} \mathrm{H}$ NMR spectrum of acetic acid is shown in Figure 3.25, along with confirmation of the compound in vaporized (PG/GL) by minute addition. 


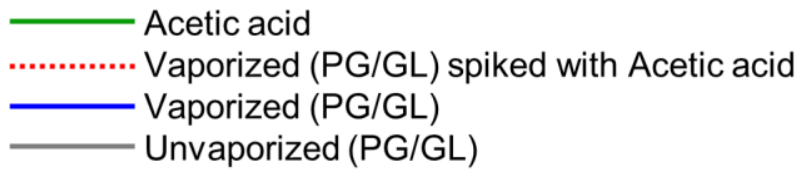

Vaporized (PG/GL) spiked with Acetic acid

Vaporized (PG/GL)

Unvaporized (PG/GL)
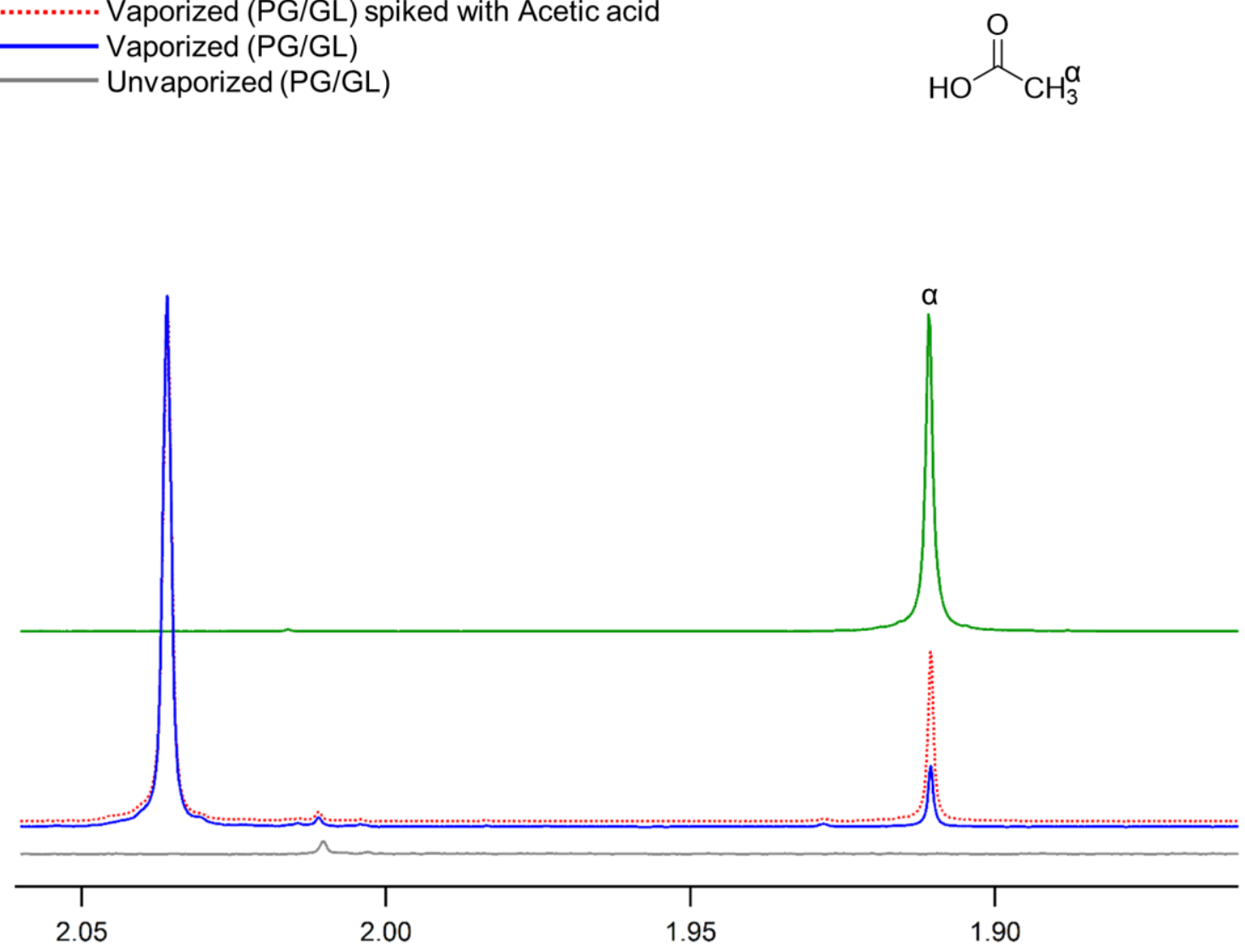

Figure 3.25 $-{ }^{1} \mathrm{H}$ NMR Assignment of Acetic Acid and Confirmation in Vaporized (PG/GL).

Glycerol itself contains no methyl carbon - only methylenes and a methyne - so acetic acid must be a secondary decomposition product of hydroxyacetone or some other compound that has already formed a methyl carbon by dehydration and rearrangement, or it must result from a multi-step process where such a rearrangement occurs.

\subsection{Formic Acid and Glycerol- $\beta$-Formate}

The structure of formic acid is shown in Figure 3.26. 


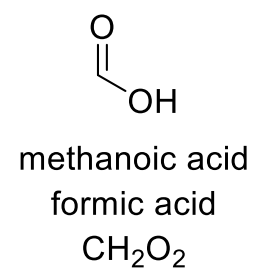

Figure 3.26 - Molecular Structure of Formic Acid.

Formic acid has a sharp singlet (s, $8.14 \mathrm{ppm})$ corresponding to the aldehyde proton. The partially assigned ${ }^{1} \mathrm{H}$ NMR spectrum of formic acid is shown in Figure 3.27, along with confirmation of the compound in vaporized (PG/GL) by minute addition.
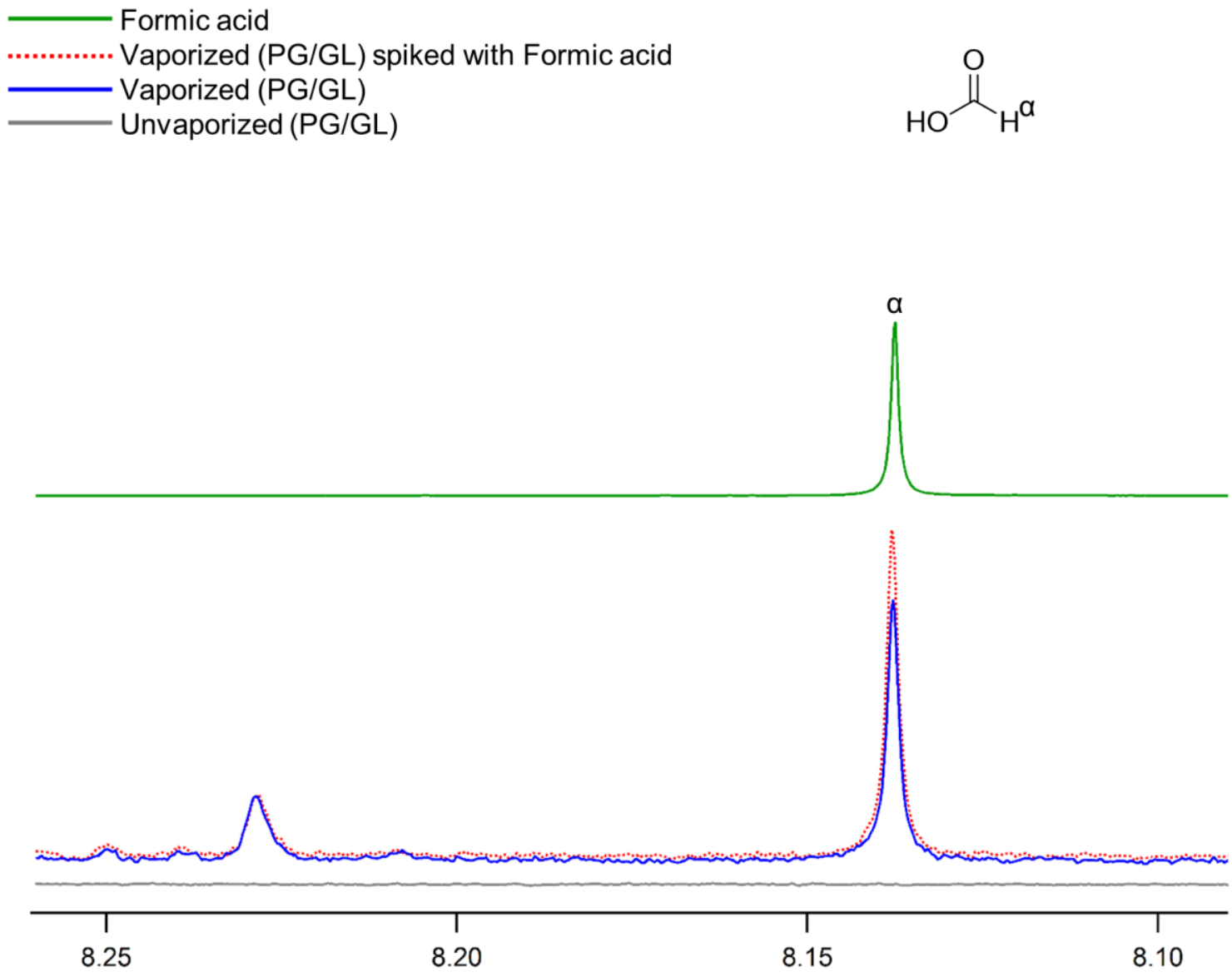

Figure 3.27 $-{ }^{1} \mathrm{H}$ NMR Assignment of Formic Acid and Confirmation in Vaporized (PG/GL). 
Formic acid may result from elimination of a carbon-carbon bond by a glycerol oxy-alkyl radical, separating from the other two carbons, which may rearrange into a stable product such as glycolaldehyde or acetaldehyde, or may further decompose into volatile singlecarbon products such as formaldehyde, carbon monoxide, or carbon dioxide. Formic acid is known to form formate esters by condensation with alcohols such as glycerol and propylene glycol; according to Miner and Dalton, ${ }^{1}$ reaction with glycerol is favored at the $\beta$-hydroxyl. This reaction is shown in Scheme 3.6.
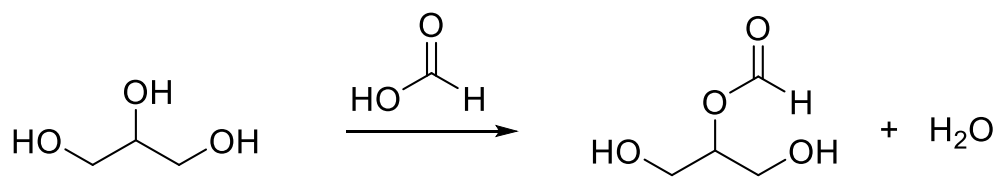

Scheme 3.6 - Formation of Glycerol $\beta$-Formate.

It is hypothesized that the small peak near $8.23 \mathrm{ppm}$ in the spectra of the vaporized (PG/GL) samples plotted in blue and red in Figure 3.27 represents the formyl proton of glycerol $\beta$-formate.

\subsubsection{Glycerol Decomposition Summarized}

Glycerol decomposes primarily into the oxidation products dihydroxyacetone and glyceraldehyde and the dehydration products glycidol, hydroxyacetone, and 3hydroxypropanal. Hydroxyacetone is known to further dissociate into formaldehyde and acetaldehyde, while 3-hydroxypropanal further dehydrates into acrolein. Glyceraldehyde may dissociate into formaldehyde and glycolaldehyde (which itself may dissociate into two 
formaldehyde monomers). Formic acid may result from C-C bond elimination of oxy-alkyl radicals, while allyl alcohol and acetic acid result as products of secondary decomposition reactions. The thermal decomposition of glycerol in the presence of oxygen is summarized in Scheme 3.7.

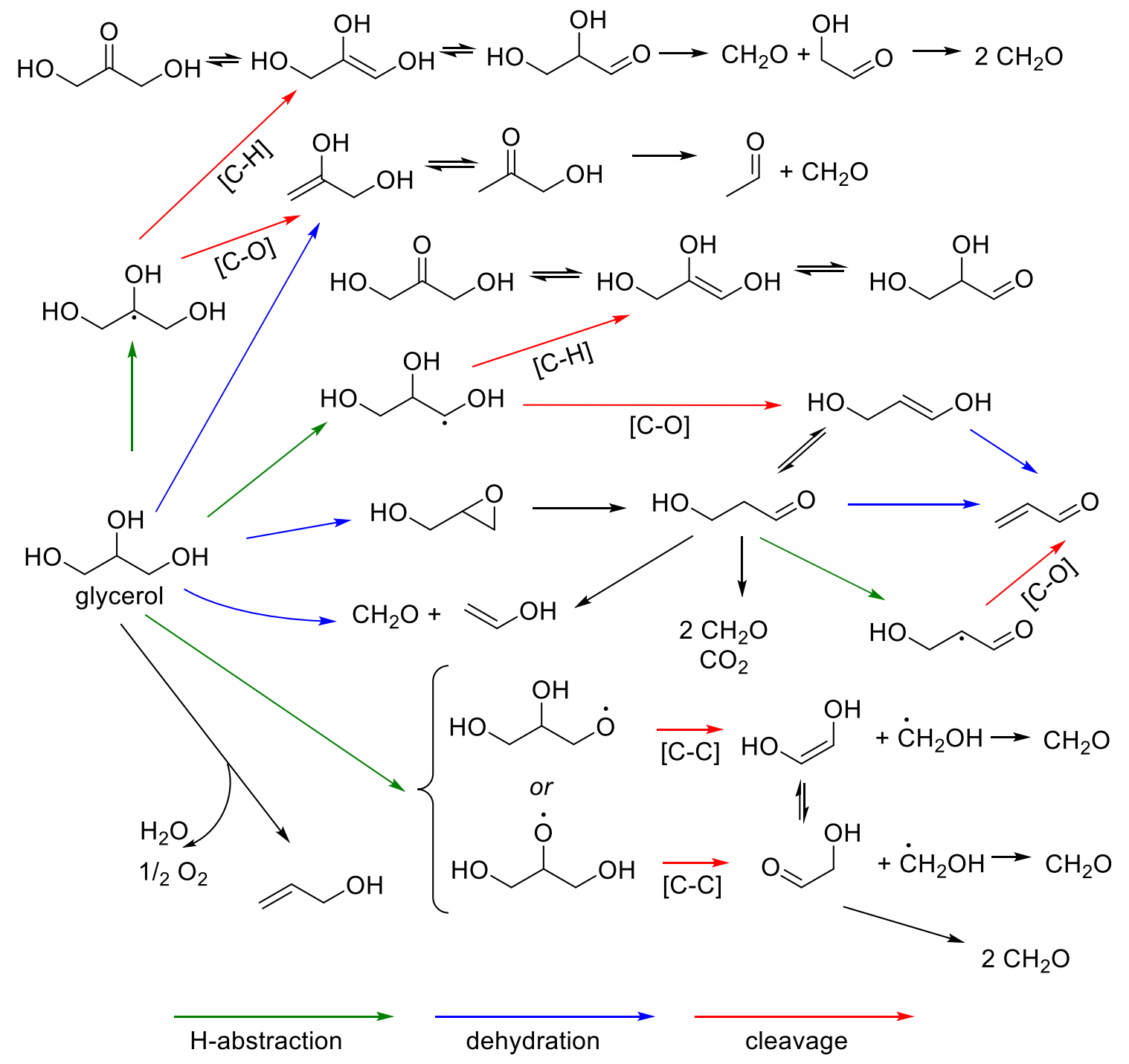

Scheme 3.7 - Aerobic Thermal Decomposition of Glycerol. 


\subsubsection{Propylene Glycol Degradation}

\subsubsection{Propylene Glycol Dehydration}

In his 1904 publication in Liebigs Annalen der Chemie, ${ }^{44}$ John Nef reported collecting 11.5 $\mathrm{mL}$ of feuchtem Propylaldehyd, völlig frei von Aceton, or "damp propanal, completely free of acetone," after heating $26 \mathrm{~g}$ of propylene glycol at $500{ }^{\circ} \mathrm{C}$ in a pumice-filled combustion tube. Nef had to synthesize propylene glycol himself, which he did by treating propylene oxide with water; in the same article he discussed the formation of glycidol by dehydration of glycerol, showing that he was well aware that oxirane rings combine with water to form glycols. The fact that he did not observe acetone in his sample of decomposed propylene glycol made him suspect that propylene oxide might not be an intermediate in the dehydration of propylene glycol, for he knew that propylene oxide formed both aldehyde and ketone products upon ring opening. Modern articles published by Dai et al. and Laino et al. propose reaction pathways using enols and propylene oxide as intermediates, ${ }^{67,101}$ very similar to Nef's discussion of glycerol dehydration. These pathways are summarized in Scheme 3.8.

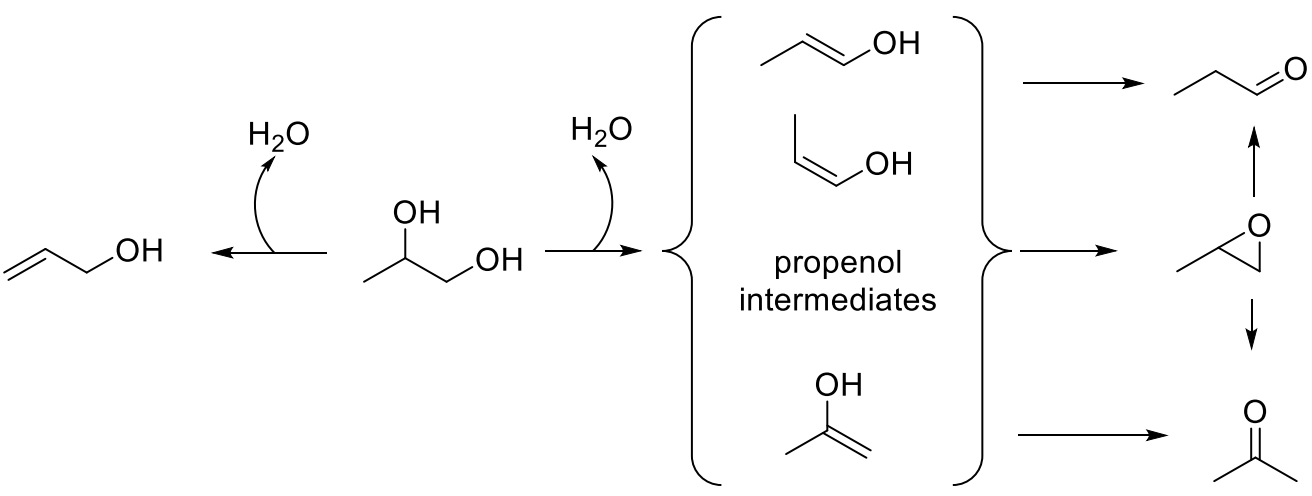

Scheme 3.8 - Dehydration of Propylene Glycol as Discussed by Nef, Laino et al., and Dai et al. 
The above pyrolysis products of propylene glycol are discussed from the perspective of anoxic dehydration routes, but may also be generated by oxygen-insertion followed by hydrogen-abstraction and C-O bond elimination as discussed in Section 3.4.3.2.

\subsection{Propanal}

The structure of propanal is shown in Figure 3.28.

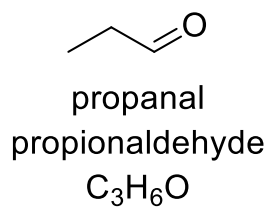

Figure 3.28 - Molecular Structure of Propanal.

Propanal has three ${ }^{1} \mathrm{H}$ resonances that are visible in samples of vaporized (PG/GL) corresponding to the aldehyde (t, $9.68 \mathrm{ppm})$, the $\alpha$-methylene protons (dq, $2.45 \mathrm{ppm}$ ), and the methyl protons (t, $0.96 \mathrm{ppm})$ - the methyl triplet is sometimes occluded by the overwhelmingly-large PG methyl triplet of similar chemical shift. Unless samples are very concentrated, the doublet-of-quartets corresponding to the $\alpha$-methylene protons is not fully resolved from the DMSO- $\mathrm{D}_{5} \mathrm{H}$ peak at $2.50 \mathrm{ppm}$. The partially assigned ${ }^{1} \mathrm{H}$ NMR spectrum of propanal is shown in Figure 3.29, along with confirmation of the compound in vaporized (PG/GL) by minute addition. 

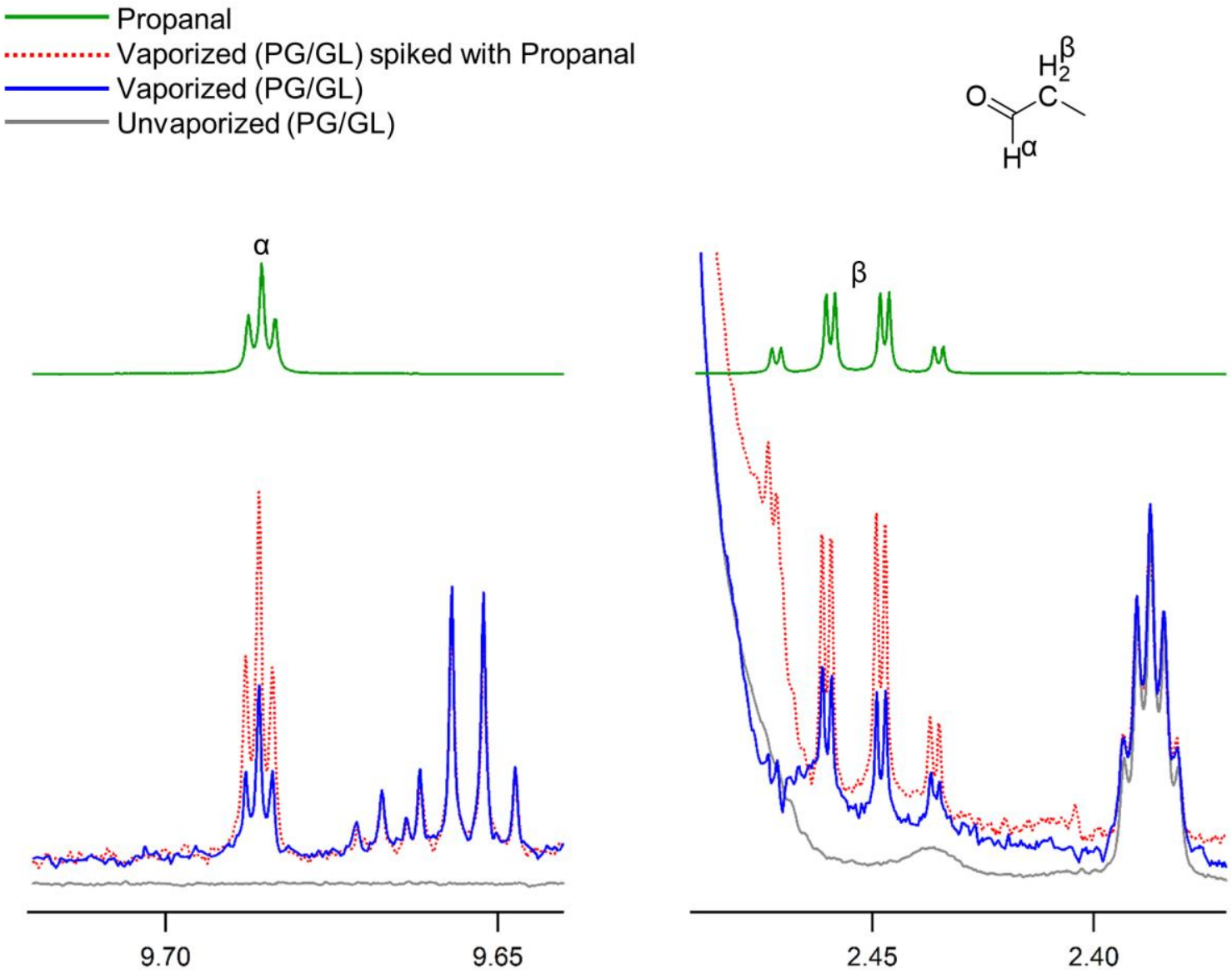

Figure 3.29 - ${ }^{1} \mathrm{H}$ NMR Assignment of Propanal and Confirmation in Vaporized (PG/GL).

\subsection{E,Z-prop-1-ene-1-ol}

The structures of the $E$ and $Z$ isomers of prop-1-ene-1-ol are shown in Figure 3.30. 

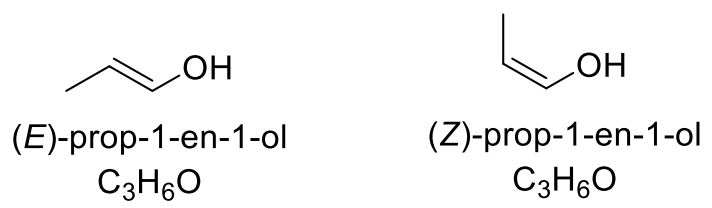

Figure 3.30 - Molecular Structures of E,Z-prop-1-ene-1-ol.

These vinyl alcohols are enols - unstable intermediates that form during the dehydration of propylene glycol and tautomerize to form propanal. The observation of these compounds by ${ }^{1} \mathrm{H}$ NMR spectroscopy has been described in the literature, ${ }^{102}$ and they have been proposed as reasonable intermediates of propylene glycol decomposition, ${ }^{77,101}$ but their direct observation in pyrolyzed samples of propylene glycol has not been previously reported as far as I am aware. The conditions under which samples are collected and analyzed in this investigation (see Section 3.2) leads to the rapid dilution of vaporized (PG/GL) and their degradation products, allowing reactive species such as these prop-1ene-1-ols to persist for hours or days before rearranging into the more stable carbonyl compound. This rearrangement can be observed by ${ }^{1} \mathrm{H}$ NMR experiments and can be accelerated by heating if the probe being used is capable of controlling and varying temperature. The separation and detection of these vinyl alcohols by gas chromatography may be difficult due to their tautomerization to propanal unless care is taken to keep the inlet and oven temperatures low. The ${ }^{1} \mathrm{H}$ NMR assignments of E,Z-prop-1-ene-1-ol and evidence of their tautomerization to propanal are summarized in Figure 3.31. In this figure, unvaporized propylene glycol is plotted in grey, indicating that all the peaks shown are degradation products; in blue is plotted a spectrum of vaporized propylene glycol collected immediately after sample collection, and in red is plotted a spectrum of that same sample 
collected after 24 hours at $25^{\circ} \mathrm{C}$. Resonances corresponding to the enols are plotted on top and diminish over time, while those corresponding to propanal (for which ${ }^{1} \mathrm{H}$ NMR assignments are given in Figure 3.29) are plotted on bottom and grow over time.
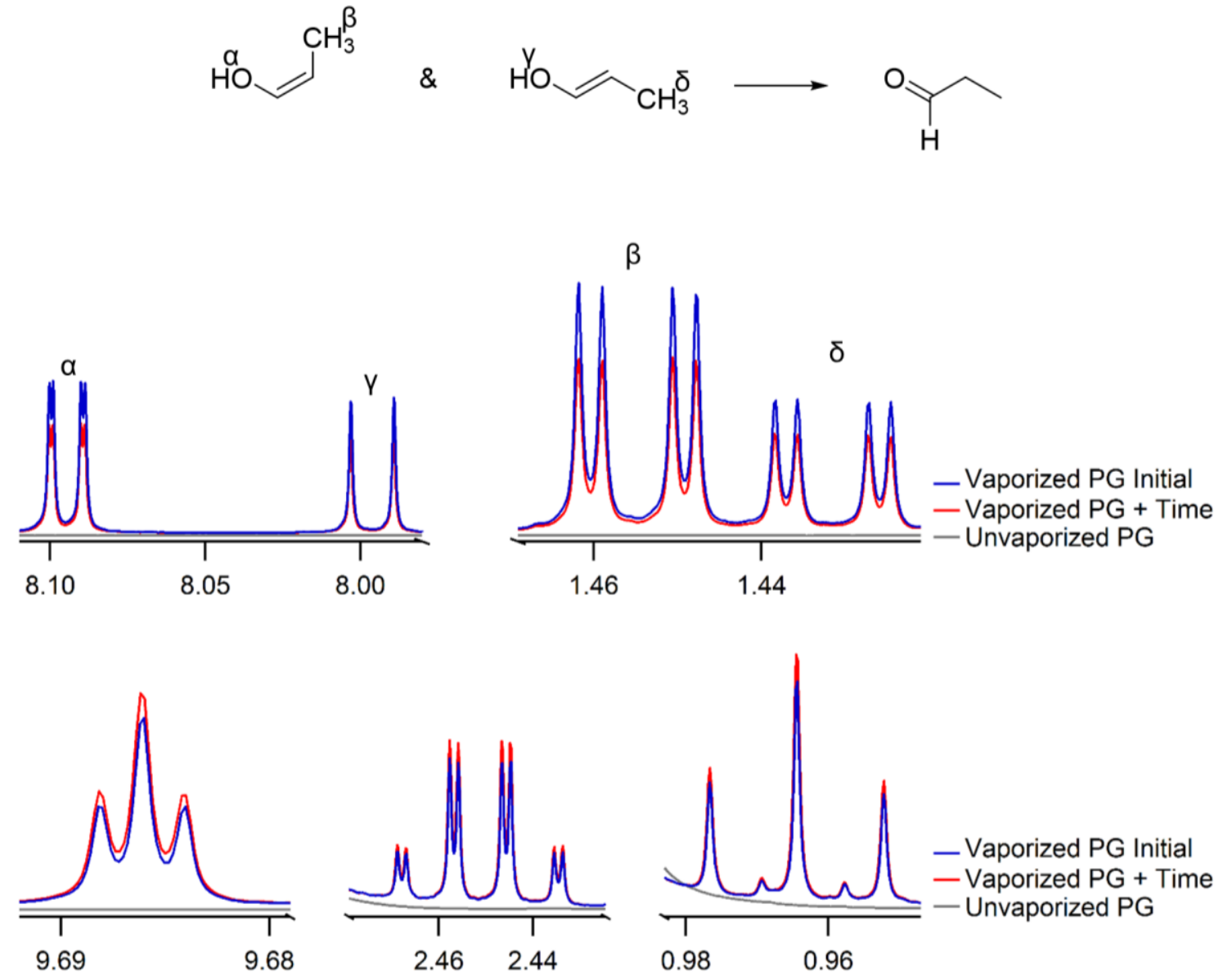

Figure 3.31 - Tautomerization of E,Z-prop-1-ene-1-ol to Propanal by ${ }^{1} \mathrm{H}$ NMR Spectroscopy.

\subsection{Acetone}

In their 2010 article discussing the oxidative thermal decomposition of propylene glycol, ${ }^{77}$ authors Díaz et al. reported acetone was the dominant three-carbon product, not propanal as Nef had found. The structure of acetone is shown in Figure 3.32. 


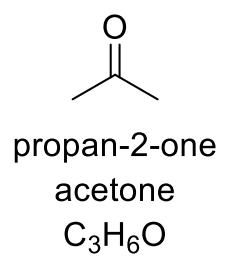

Figure 3.32 - Molecular Structure of Acetone.

The compound is symmetrical and has only a single ${ }^{1} \mathrm{H}$ resonance (s, $2.09 \mathrm{ppm}$ ) making it particularly unobvious in a spectrum of vaporized (PG/GL) containing dozens of peaks. This resonance can be correlated to an acetyl carbon and a ketone using HSQC and HMBC experiments; the only way to confirm that this resonance is from acetone specifically is to spike the sample with a minute addition of the pure compound. The assigned ${ }^{1} \mathrm{H}$ NMR spectrum of acetone is shown in Figure 3.33, along with confirmation of the compound in vaporized (PG/GL) by minute addition. 

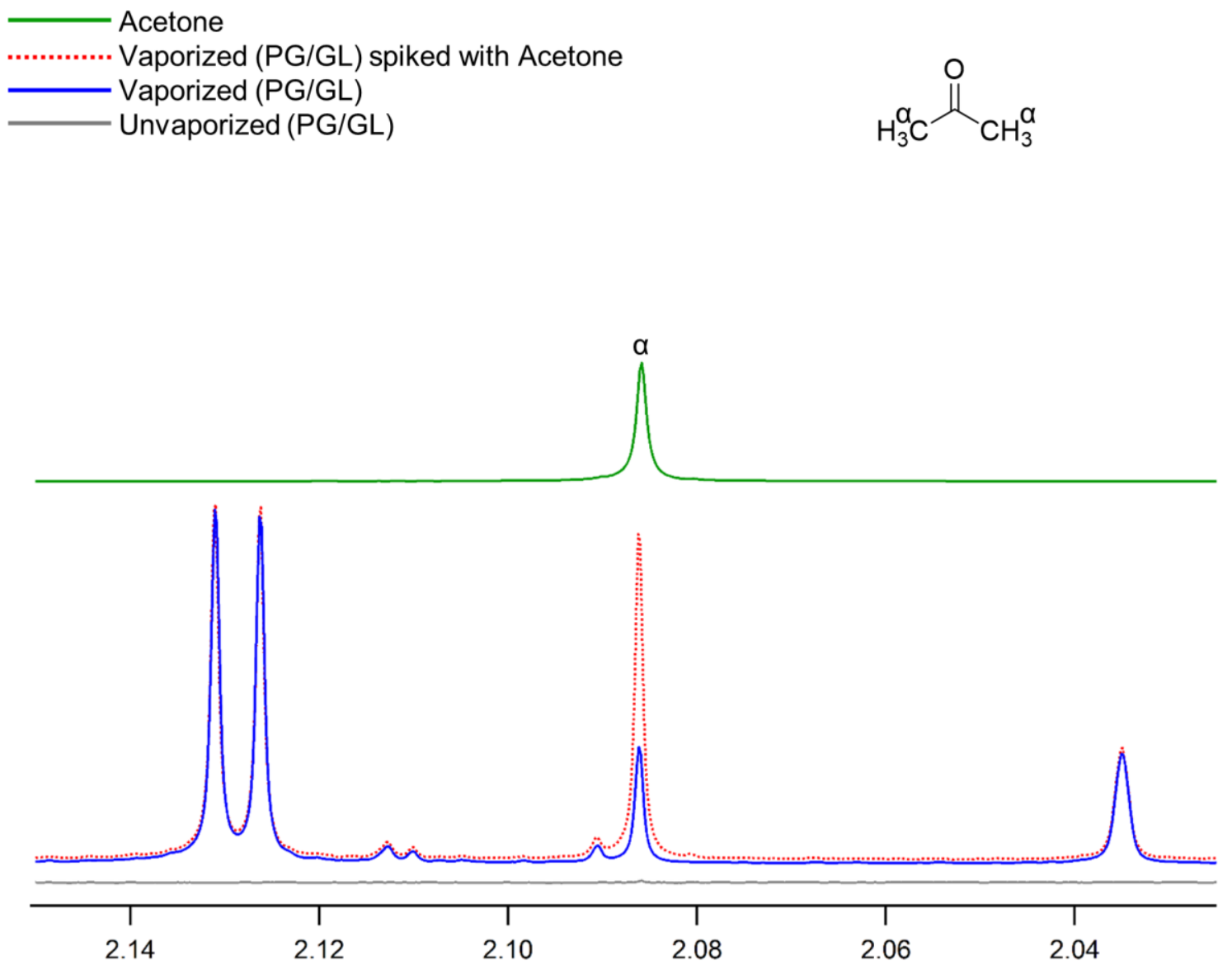

Figure 3.33 - ${ }^{1} \mathrm{H}$ NMR Assignment of Acetone and Confirmation in Vaporized (PG/GL).

\subsection{Allyl Alcohol}

Allyl alcohol is a dehydration product of propylene glycol that might also form by C-O elimination of an alkyl radical after oxygen insertion and hydrogen abstraction. ${ }^{67,77}$ The structure and ${ }^{1} \mathrm{H}$ NMR spectrum of allyl alcohol is discussed in Section 3.4.2.3.2 under Further Glycerol Dissociation. 


\subsubsection{Propylene Glycol Oxidation}

The oxidation of propylene glycol in air may be initiated by insertion of $\mathrm{O}_{2}$ into the $\mathrm{C}-\mathrm{H}$ bond of the $\alpha-, \beta-$, or $\gamma$-carbon. The resulting hydroperoxyl compound may decompose to form a hydroperoxyl radical and an alkyl radical, or alternatively a hydroxyl radical and an oxy-alkyl radical, all of which propagate. By abstraction of an additional hydrogen atom, such as the $\mathrm{C}-\mathrm{H}$ of an unreacted equivalent of propylene glycol, hydroxyl radical forms water and hydroperoxyl radical forms hydrogen peroxide, both leaving behind another alkyl radical, as depicted in Scheme 3.9, an original composition borrowing from Diaz et al. and Comprehensive Chemical Kinetics Vol. 17: Gas Phase Combustion.,77

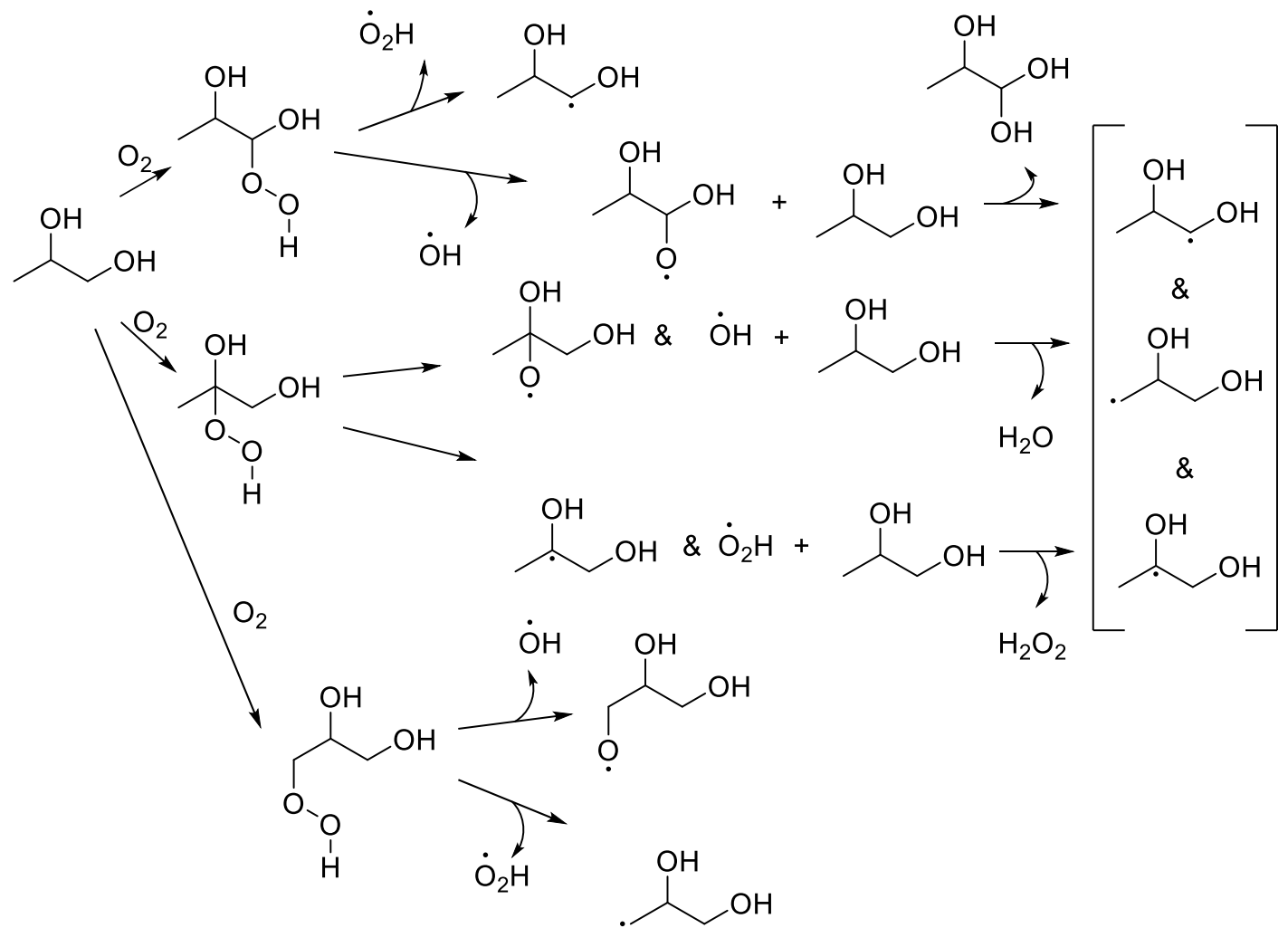

Scheme 3.9 - Initiation of Propylene Glycol Oxidation. 
Scheme 3.10 shows the insertion of $\mathrm{O}_{2}$ into the $\mathrm{C}-\mathrm{H}$ bond of the $\alpha-, \beta-$, and $\gamma$-carbons of propylene glycol to form hydroperoxide compounds, which lose hydroxyl radical and form oxy-alkyl radicals. These $\alpha-, \beta$-, and $\gamma$-oxy-alkyl radicals might abstract a hydrogen and form a triol, or cleave bonds and form a diverse array of stable decomposition products while further propagating an equally diverse array of radicals.

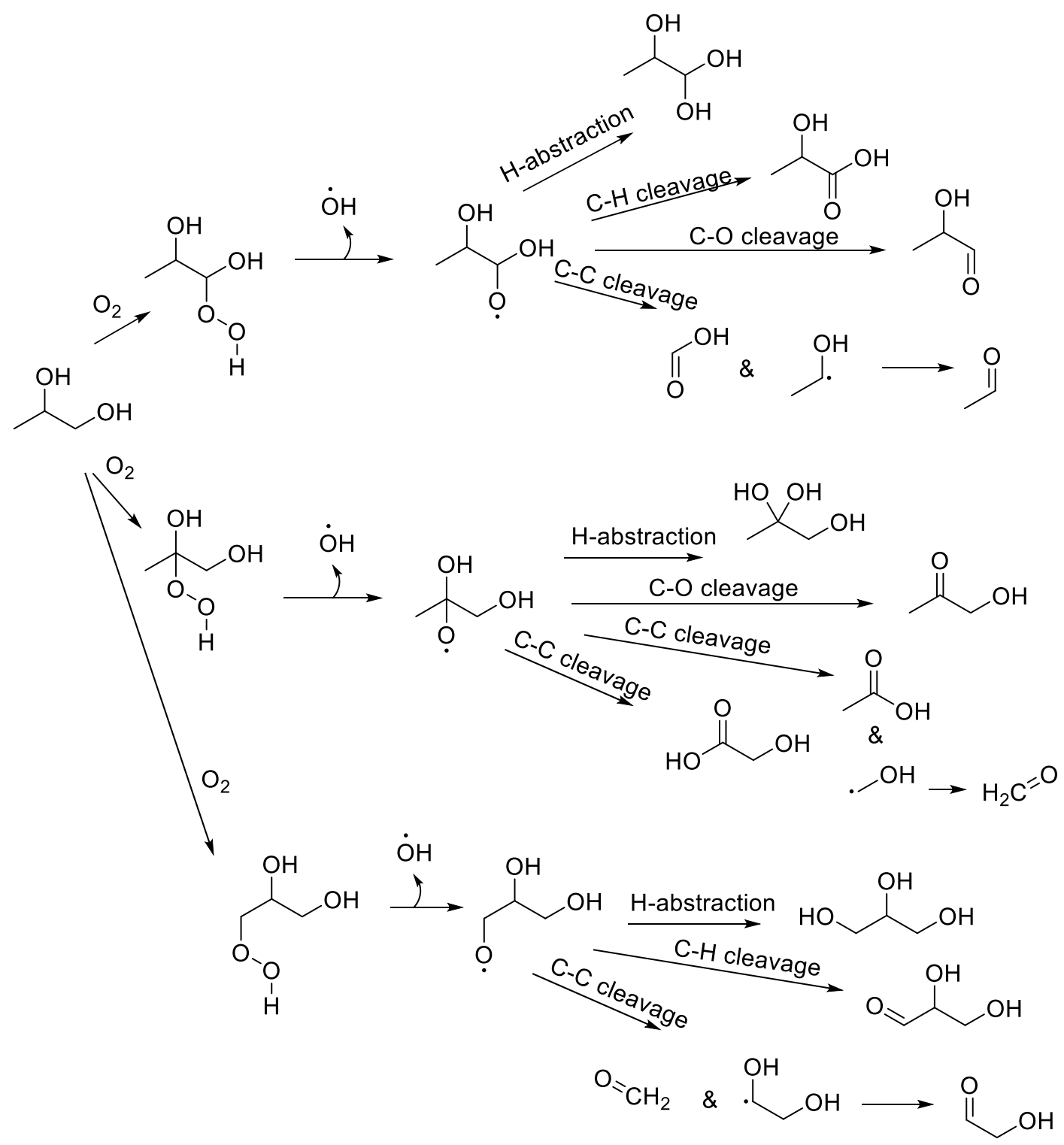

Scheme 3.10 -Propylene Glycol Oxidation - Oxy-alkyl Radicals. 
The insertion of oxygen between the $\mathrm{C}-\mathrm{H}$ bond of the $\gamma$-carbon of propylene glycol and formation of the subsequent $\gamma$-oxy-alkyl radical is unlikely; the formation of glycerol, glyceraldehyde, and glycolaldehyde have not been observed in vaporized samples of pure propylene glycol.

The hydroxyl-alkyl radicals formed by abstraction of hydrogen from the $\alpha$ - and $\beta$-carbons of propylene glycol may decompose by elimination of one of the $\mathrm{C}-\mathrm{O}$ or $\mathrm{C}-\mathrm{H}$ bonds. Elimination of one of the $\mathrm{C}-\mathrm{O}$ bonds forms enols which tautomerize to the corresponding ketone or aldehyde product - acetone and propanal. Elimination of one of the $\mathrm{C}-\mathrm{H}$ bonds results in a di-enol intermediate which undergoes tautomerization to form hydroxyacetone and lactaldehyde, which are simple oxidation products of propylene glycol (meaning that propylene glycol has lost $\mathrm{H}_{2}$ during the reaction). The alkyl radical formed by abstraction of hydrogen from the $\gamma$-carbon of propylene glycol may decompose by $\mathrm{C}$-O elimination to form allyl alcohol or C-H elimination to form hydroxyacetone, but may also undergo C-C elimination and produce acetaldehyde and formaldehyde. The elimination, tautomerization, and secondary decomposition reactions discussed above are summarized in Scheme 3.11. 


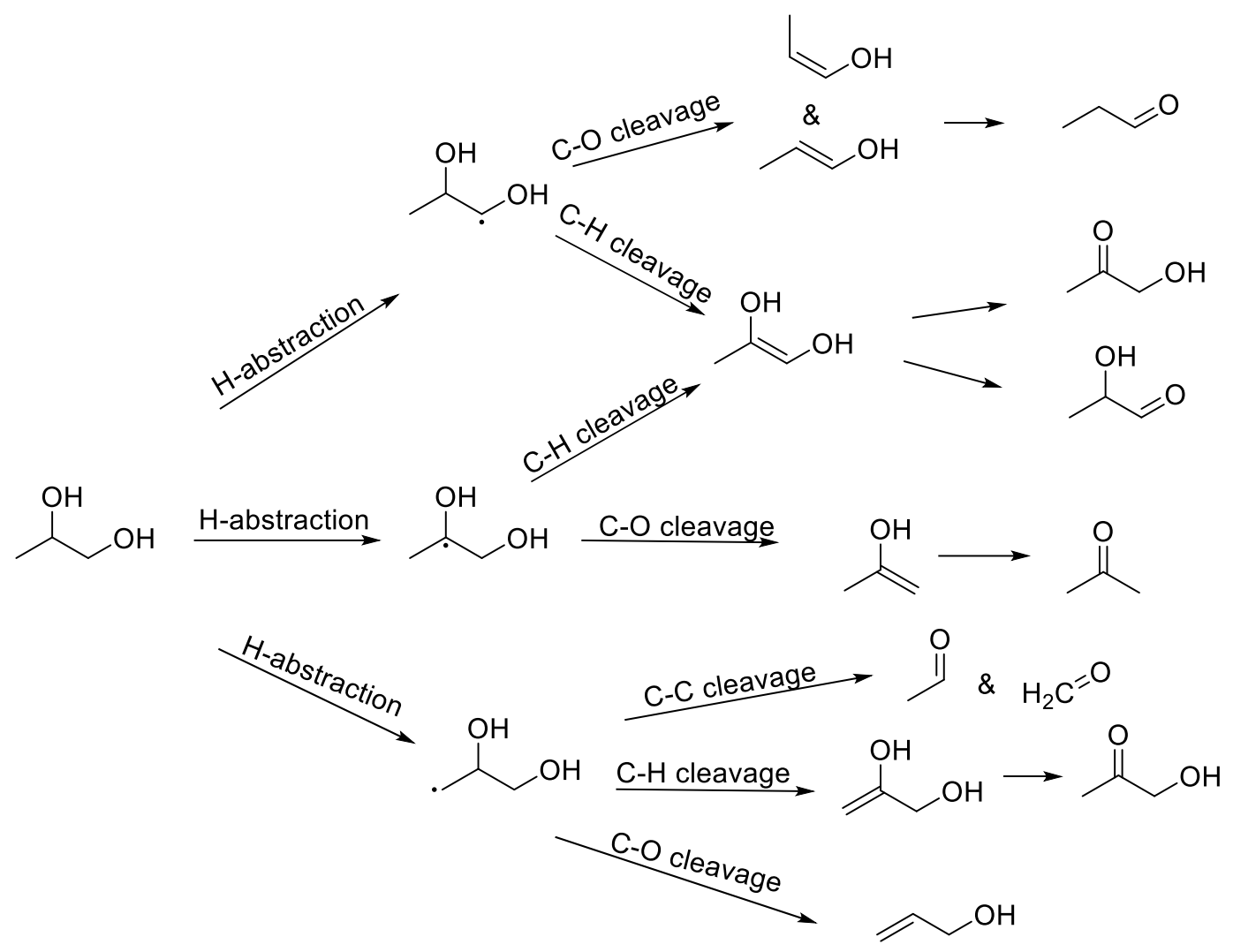

Scheme 3.11 - Propylene Glycol Oxidation - Alkyl and Hydroxyl-alkyl Radicals.

\subsection{Hydroxyacetone}

Hydroxyacetone is a primary oxidation product of propylene glycol and a primary dehydration product of glycerol. Confirmation of this compound in samples of vaporized (PG/GL) and the corresponding ${ }^{1} \mathrm{H}$ NMR spectrum assignments are discussed in Section

\subsubsection{1 under Glycerol Dehydration.}

\subsection{Lactaldehyde}

The structure of lactaldehyde is shown in Figure 3.34. 


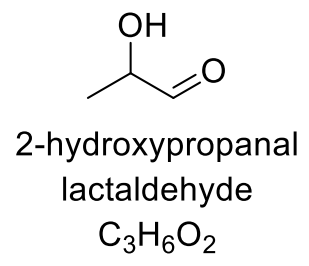

Figure 3.34 - Molecular Structure of Lactaldehyde.

Lactaldehyde is an $\alpha$-hydroxyaldehyde - like glyceraldehyde, the aldehyde resonance is a very tightly coupled doublet. Perhaps unsurprisingly, most of the non-aldehyde protons present in lactaldehyde resonate at frequencies very similar to (PG/GL) and are not well resolved. The partially assigned ${ }^{1} \mathrm{H}$ NMR spectrum of lactaldehyde is shown in Figure 3.35, along with confirmation of the compound in vaporized (PG/GL) by minute addition. 


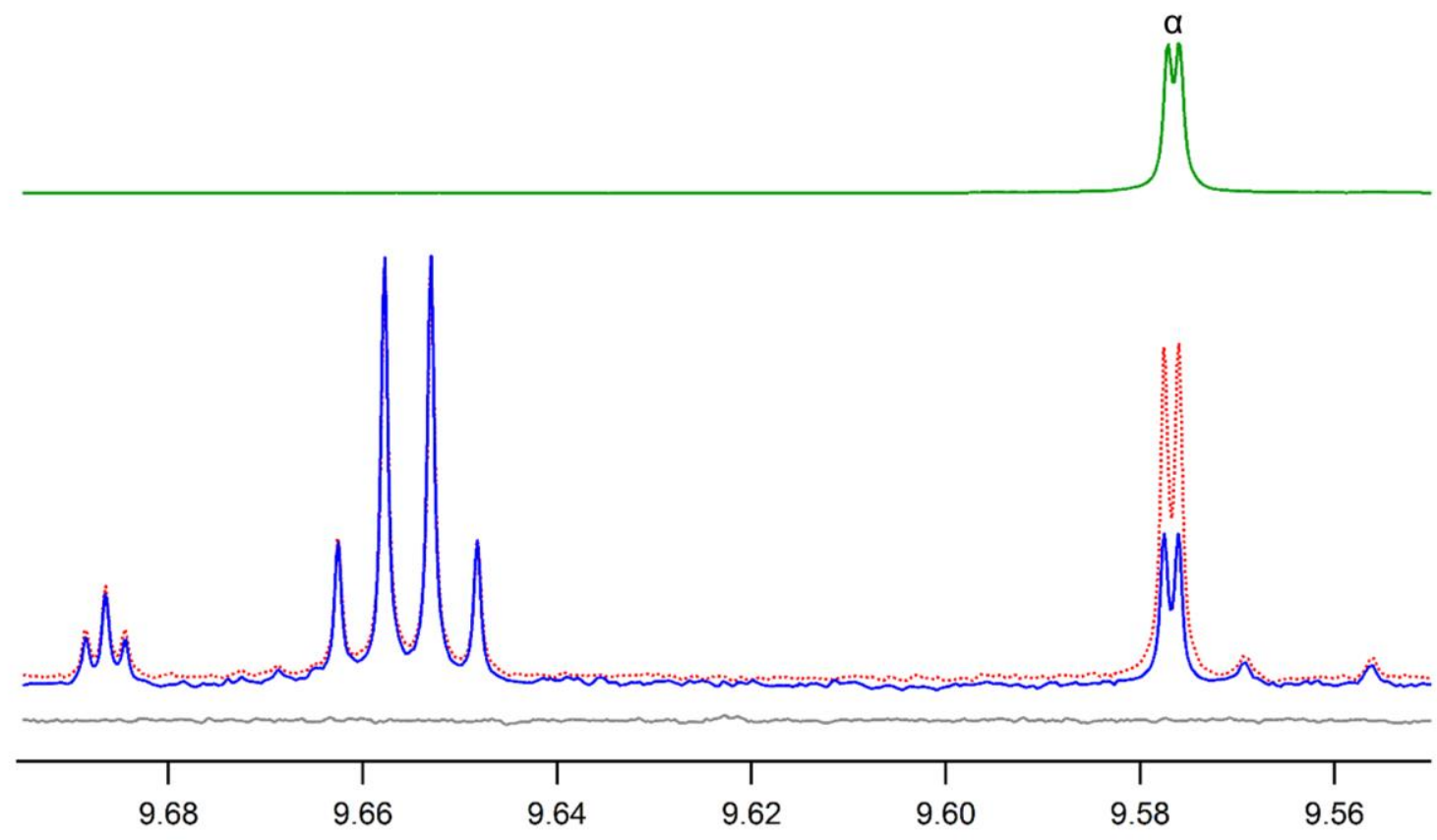

Figure 3.35 $-{ }^{1} \mathrm{H}$ NMR Assignment of Lactaldehyde and Confirmation in Vaporized (PG/GL).

\subsubsection{Further Propylene Glycol Dissociation}

Like glycerol, propylene glycol decomposition may result in the formation of secondary reaction products including acetaldehyde and formaldehyde, which may form as products of hydroxyacetone dissociation or as C-C elimination products of an alkyl radical. Other small molecules are also observed in samples of vaporized propylene glycol, including acetic acid and formic acid, both of which may form by C-C elimination of oxy-alkyl radicals. Lactaldehyde is capable of dehydration to a much smaller degree than 3- 
hydroxypropanal, forming a very small amount of acrolein when vaporized at high temperatures. The small peaks of the acrolein aldehyde doublet can be seen from .57 to $9.55 \mathrm{ppm}$ in the spectrum of vaporized propylene glycol plotted in blue and red in Figure 3.35 .

\subsubsection{Propylene Glycol Decomposition Summarized}

Propylene glycol decomposes primarily into the oxidation products hydroxyacetone and lactaldehyde and the dehydration products acetone, allyl alcohol, and propanal. Reactive enol intermediates E,Z-prop-1-ene-1-ol have been identified as precursors to propanal. Hydroxyacetone is known to further dissociate into formaldehyde and acetaldehyde, while lactaldehyde can further dehydrate into acrolein to a limited degree. Acetic acid and formic acid may result from C-C bond elimination of oxy-alkyl radicals. The thermal decomposition of propylene glycol in the presence of oxygen is summarized in Scheme 3.12 . 


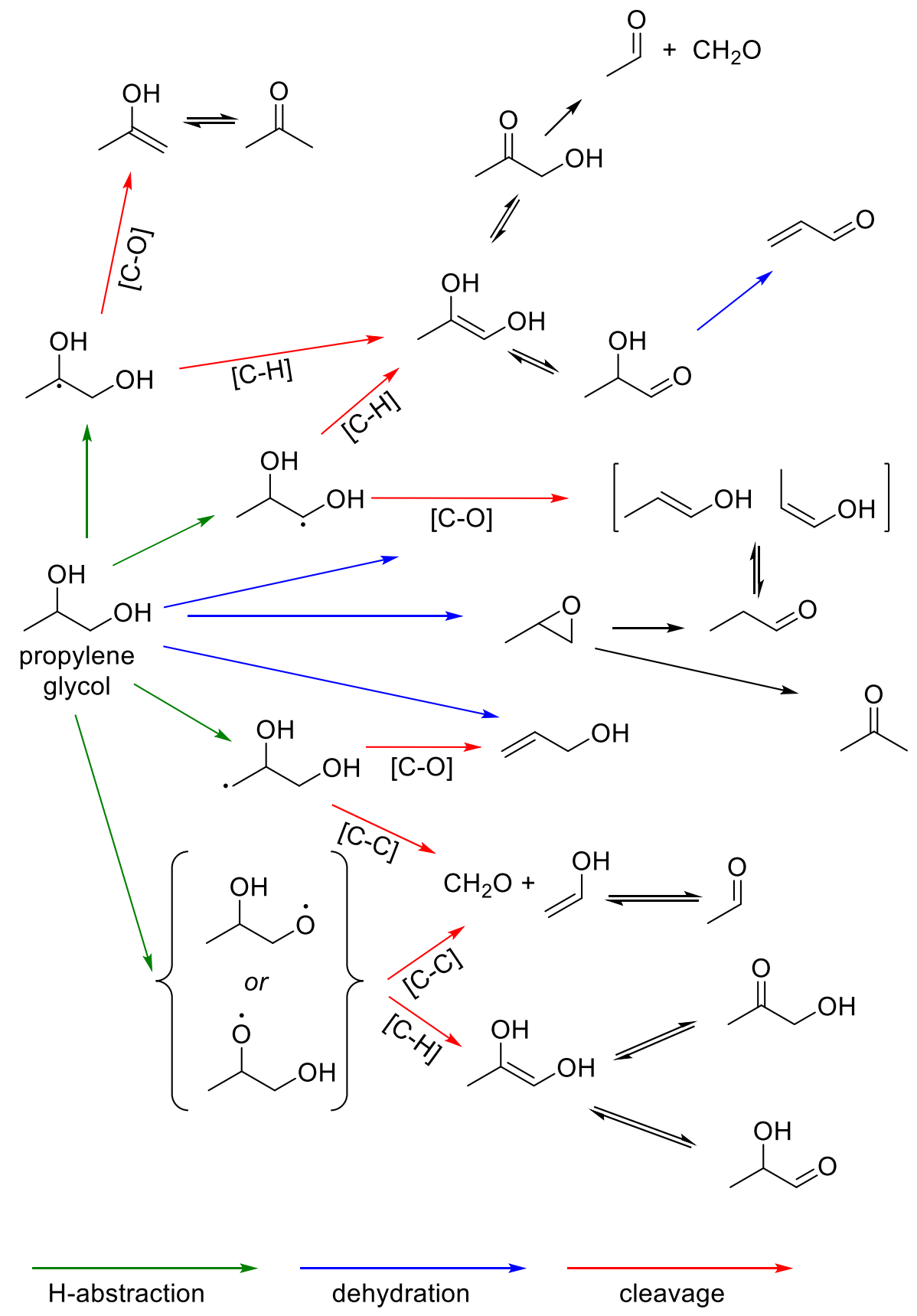

Scheme 3.12 - Aerobic Thermal Decomposition of Propylene Glycol. 


\subsection{Quantification of Decomposition Products}

The abundance of a decomposition product in a sample of vaporized (PG/GL) is dependent upon the operating power of the e-cigarette, the rate of air flowing over the heating coil during the puff, and the surface of the heating coil used to vaporize the sample. Along with these factors, a number of additional concerns complicate the process of developing a quantitative understanding of (PG/GL) decomposition. If the specific product under investigation is a primary decomposition product occurring early in a degradation pathway (such as hydroxyacetone or glycidol) then the abundance of the product may decline as the reactivity of the system is increased by increasing temperature. Moreover, the sample collection technique employed in this investigation does not retain a consistent portion of the (PG/GL) consumed from the reservoir. Downstream decomposition products such as acetaldehyde, formaldehyde, carbon monoxide, ethylene, and carbon dioxide are volatile and might be poorly retained using the flow-through technique discussed in Section 3.2. Detection of gases like carbon monoxide and carbon dioxide cannot be achieved by ${ }^{1} \mathrm{H}$ NMR spectroscopy, making it impossible to analyze the entirety of a highly degraded vaporized sample by this method. Even if there existed some clearly defined average of

user behaviors by which to standardize experimental parameters like operating power, puff duration, and puff volume, the unique features and aspects of each individual heating element would still present a major obstacle in attempting to generalize the extent of decomposition beyond the context of the device under investigation.

Nevertheless, it is possible to quantify decomposition products within samples of vaporized (PG/GL) analyzed by ${ }^{1} \mathrm{H}$ NMR spectroscopy if experiments are carefully conducted. The 
information collected from such experiments could be useful in developing a deeper mechanistic understanding of (PG/GL) decomposition if steps were taken to properly address the problems of sample retention discussed above. Even without addressing those problems, quantification of certain specific decomposition products of (PG/GL) such as hydroxyacetone or acetaldehyde can be sufficiently straightforward that simple trends might be observed, including increased abundance with increased operating power or increased duration of a $50 \mathrm{~mL}$ puff. In the experiment discussed below, these trends were demonstrated by collecting samples under fixed conditions while varying the operating power and puff duration - variables that were shown in Section 3.3 to affect the mass of (PG/GL) consumed from the reservoir and are suspected to correspond to the temperature of the heating element.

\subsubsection{Quantitative Sample Collection}

The samples collected and analyzed in this experiment are outlined in Table 3.1. Each sample consisted of three $50 \mathrm{~mL}$ puffs of (PG/GL) vaporized in ascending order at 6, 8, 10, 12 , and 14 Watts over the duration of 3, 5, and 10 second puffs for a total of 15 samples composed of 45 puffs. The cartomizer was removed from the e-cigarette and weighed on an analytical balance before and after each of the three puffs taken per sample in order to determine the mass of (PG/GL) consumed from the reservoir during each puff. In this experiment, three identical KangerTech ${ }^{\circledR}$ Protank II cartomizers were used to collect triplicate sets of samples. Each cartomizer was equipped with a unique, identical $2.2 \Omega$ coil and each fitted to one of three identical Innokin ${ }^{\circledR}$ iTaste V4 batteries. These triplicate sets of samples taken from identical devices are intended to account for the unique differences 
that may arise between devices, such as a poor electrical connection or a manufacturing flaw. Quantitative values determined by analysis of samples collected from these devices (designated $A, B$, and $C$ ) are presented below, first averaged together, then disjointed to show the variation between devices. The masses of (PG/GL) consumed per puff shown in Table 3.1 are the average $+/$ - standard deviation of nine identical puffs (three each taken from three devices). The consumption data from Table 3.1 is plotted in Figure 3.36, representing 45 samples composed of 135 puffs, taken from 3 different devices.

Table 3.1 - Conditions and Consumption of Samples for Quantification of Degradation Products.

\begin{tabular}{|c|c|c|c|c|}
\hline $\begin{array}{c}\text { Collection } \\
\text { Order } \\
\text { (Sample \#) }\end{array}$ & $\begin{array}{c}\text { E-cigarette } \\
\text { Power } \\
\text { (Watts) }\end{array}$ & $\begin{array}{c}\text { Duration of } \\
\mathbf{5 0} \mathbf{~} \mathbf{\text { puff }} \\
\text { (seconds) }\end{array}$ & $\begin{array}{c}\text { Average (n=9) Mass of } \\
\mathbf{( P G / G L )} \text { consumed from } \\
\text { reservoir per puff (grams) }\end{array}$ & $\begin{array}{c}\text { Error } \\
\text { (S.D. } \mathbf{n = 9})\end{array}$ \\
\hline $\mathbf{1}$ & 6 & 3 & 5.5 & 1.2 \\
\hline $\mathbf{2}$ & 6 & 5 & 10.1 & 1.0 \\
\hline $\mathbf{3}$ & 6 & 10 & 15.7 & 0.8 \\
\hline $\mathbf{4}$ & 8 & 3 & 8.6 & 0.9 \\
\hline $\mathbf{5}$ & 8 & 5 & 14.2 & 1.0 \\
\hline $\mathbf{6}$ & 8 & 10 & 16.6 & 1.0 \\
\hline $\mathbf{7}$ & 10 & 3 & 11.3 & 0.6 \\
\hline $\mathbf{8}$ & 10 & 5 & 17.4 & 0.8 \\
\hline $\mathbf{9}$ & 10 & 10 & 18.0 & 2.6 \\
\hline $\mathbf{1 0}$ & 12 & 3 & 13.6 & 1.3 \\
\hline $\mathbf{1 1}$ & 12 & 5 & 18.1 & 2.0 \\
\hline $\mathbf{1 2}$ & 12 & 10 & 19.5 & 3.0 \\
\hline $\mathbf{1 3}$ & 14 & 3 & 14.3 & 1.5 \\
\hline $\mathbf{1 4}$ & 14 & 5 & 19.1 & 2.1 \\
\hline $\mathbf{1 5}$ & 14 & 10 & 21.9 & 3.8 \\
\hline
\end{tabular}




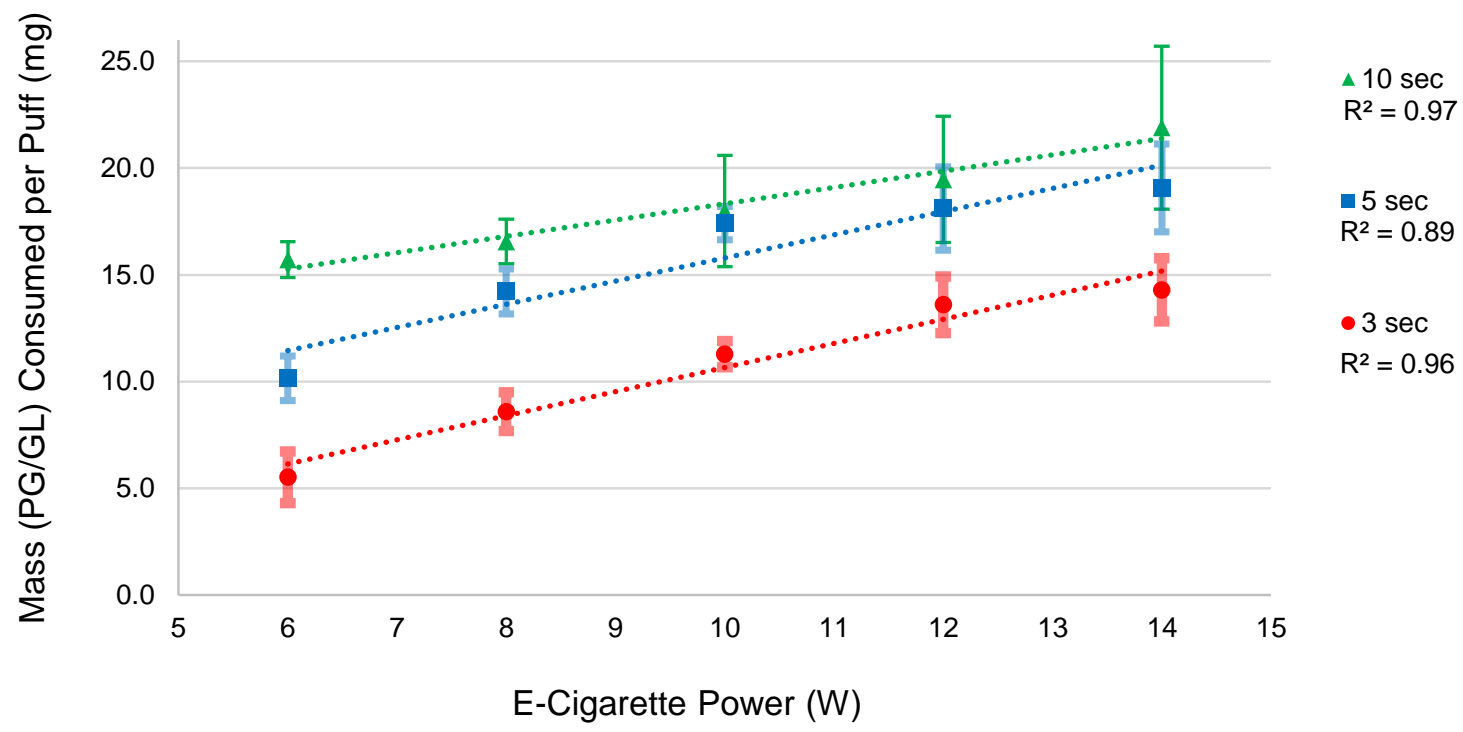

Figure 3.36 - Increased Consumption of (PG/GL) with Increased Operating Power and Puff Duration.

Similar to Figures 3.2 and 3.3 from Section 3.3, Figure 3.36 shows a general increase in the mass of (PG/GL) consumed from the reservoir with increases in both operating power and puff duration - variables that likely correlate with operating temperature. A similar relationship was hypothesized to exist between increased operating temperature and the extent of sample degradation.

\subsubsection{Materials and Methods}

The mixture of (PG/GL) used in this study was made by adding $18.4180 \mathrm{~g}$ of glycerol and $15.2180 \mathrm{~g}$ of propylene glycol (both USP grade, Sigma-Aldrich) together into a $100 \mathrm{~mL}$ bottle on an analytical balance, then capping and shaking the mixture for an extended period. The mixture is analyzed by ${ }^{1} \mathrm{H}$ NMR spectroscopy and confirmed as 1:1 molar ratio by relative integration. This (PG/GL) mixture may be added to the e-liquid reservoir of a 
cartomizer using a Pasteur pipet. Puffs of vaporized (PG/GL) were drawn through 1-inchlong, 18 gauge disposable needles into $1500 \mu \mathrm{L}$ glass vials containing $600 \mu \mathrm{L}$ of solvent for NMR analysis. These septa-capped vials were weighed both before and after collection of each three-puff sample to determine the mass of the sample collected. Collected samples were vortexed and transferred to a $5 \mathrm{~mm}$ NMR tube for analysis. Quantitative ${ }^{1} \mathrm{H}$ NMR experiments were conducted in $\mathrm{DMSO}_{6}$ (99.9\%, Cambridge Isotope) containing $0.5 \%$ v/v TMS as a chemical shift reference. For quantification by relative integration, a $1.0 \mathrm{mM}$ solution of an internal standard (1,2,4,5-tetrachloro-3-nitrobenzene, M.W. $=260.89 \mathrm{~g} / \mathrm{mol})$ was made by 1:10 dilution from a $10.0 \mathrm{mM}$ stock solution made by dissolving 0.1304 grams of the solid in a $50 \mathrm{~mL}$ volumetric flask. Samples were analyzed by ${ }^{1} \mathrm{H}$ NMR spectroscopy at 599.80 MHz using 30-degree flip angles and long inter-scan delays to ensure full relaxation. Relative integration against the internal standard was used to establish a concentration of decomposition products, which was used to determine the mass of each product within the $600 \mu \mathrm{L}$ sample. The calculated mass was then taken as a ratio of the total mass of vaporized (PG/GL) collected in the sample to determine the mass percent $(\% \mathrm{w} / \mathrm{w})$ of the product in the sample.

\subsubsection{Quantification of Hydroxyacetone and Acetaldehyde}

\subsubsection{Hydroxyacetone}

Table 3.2 shows the concentrations of hydroxyacetone in collected samples as measured by relative integration against an internal standard, along with calculated values of the mass percent of hydroxyacetone in the sample $+/$ - the standard error in the measurement - these 
values are plotted in Figure 3.37. The error terms are large relative to the calculated sample percentage, reflecting a high degree of variability between devices used in this experiment - this can be observed in Figure 3.38, which presents the calculated $\%$ w/w of hydroxyacetone in the samples generated by each of the three identical devices $(A, B$, and C) used in this experiment.

Table 3.2 - Quantification of Hydroxyacetone.

\begin{tabular}{|c|c|c|c|c|}
\hline \multirow{6}{*}{$\begin{array}{c}3 \\
\text { sec }\end{array}$} & Power (W) & $\begin{array}{c}\text { Average } \\
\text { [hydroxyacetone] }(\mathrm{mM})\end{array}$ & $\begin{array}{c}\text { Average \% } \\
\text { hydroxyacetone (w/w) }\end{array}$ & error \\
\hline & 6 & 0.020 & 0.031 & 0.015 \\
\hline & 8 & 0.090 & 0.080 & 0.062 \\
\hline & 10 & 0.393 & 0.221 & 0.124 \\
\hline & 12 & 0.920 & 0.634 & 0.404 \\
\hline & 14 & 1.160 & 0.643 & 0.427 \\
\hline \multirow{6}{*}{$\begin{array}{c}5 \\
\text { sec }\end{array}$} & Power (W) & $\begin{array}{c}\text { Average } \\
\text { [hydroxyacetone] }(\mathrm{mM})\end{array}$ & $\begin{array}{c}\text { Average \% } \\
\text { hydroxyacetone (w/w) }\end{array}$ & error \\
\hline & 6 & 0.090 & 0.036 & 0.022 \\
\hline & 8 & 0.383 & 0.157 & 0.112 \\
\hline & 10 & 0.833 & 0.403 & 0.260 \\
\hline & 12 & 1.117 & 0.578 & 0.413 \\
\hline & 14 & 1.397 & 1.012 & 0.695 \\
\hline \multirow{6}{*}{$\begin{array}{c}10 \\
\text { sec }\end{array}$} & Power (W) & $\begin{array}{c}\text { Average } \\
\text { [hydroxyacetone] }(\mathrm{mM})\end{array}$ & $\begin{array}{c}\text { Average \% } \\
\text { hydroxyacetone (w/w) }\end{array}$ & error \\
\hline & 6 & 0.450 & 0.133 & 0.098 \\
\hline & 8 & 1.020 & 0.331 & 0.184 \\
\hline & 10 & 1.297 & 1.196 & 0.742 \\
\hline & 12 & 1.777 & 0.923 & 0.587 \\
\hline & 14 & 1.710 & 1.452 & 1.026 \\
\hline
\end{tabular}




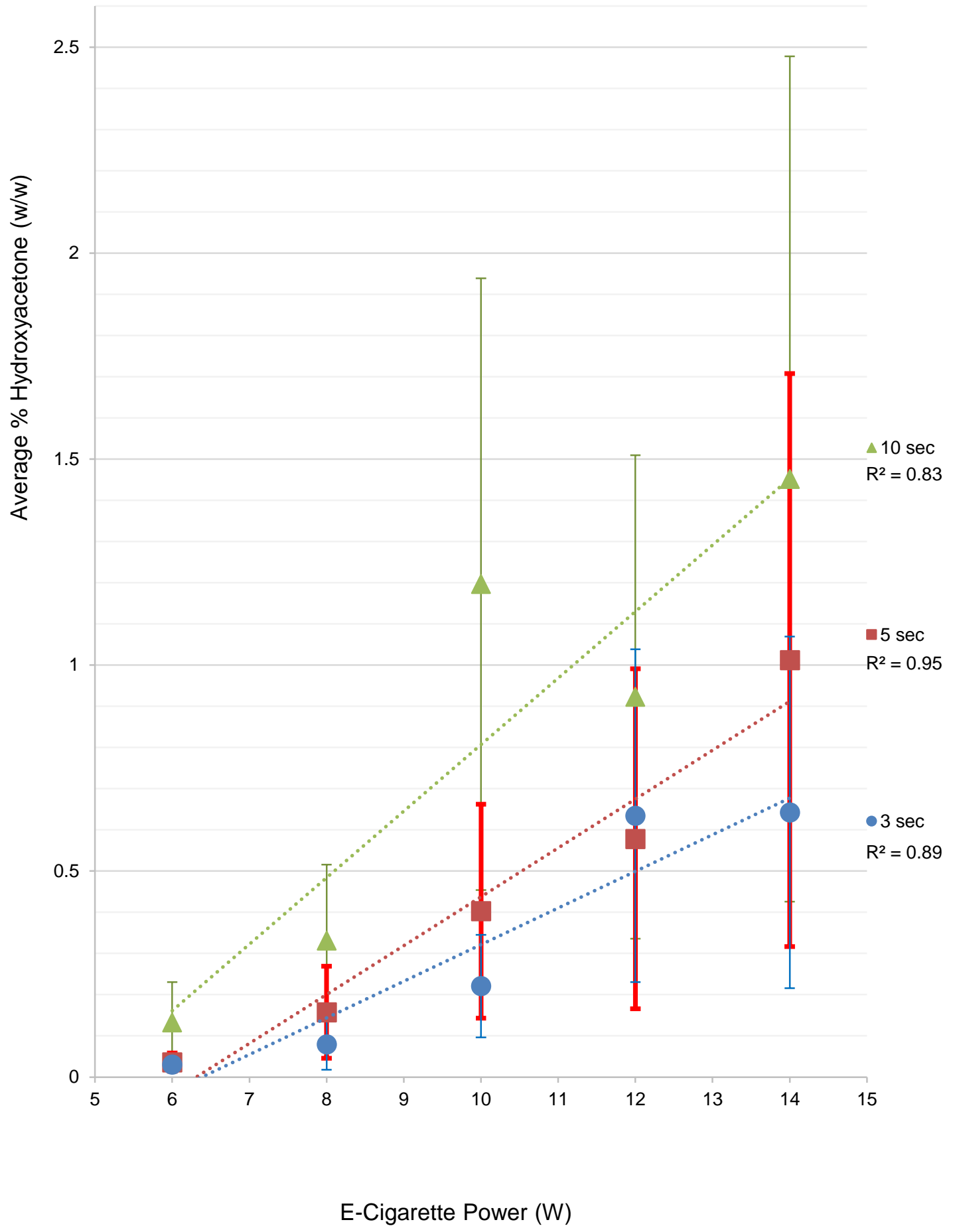

Figure 3.37 -Hydroxyacetone Values Averaged from Three Devices. 

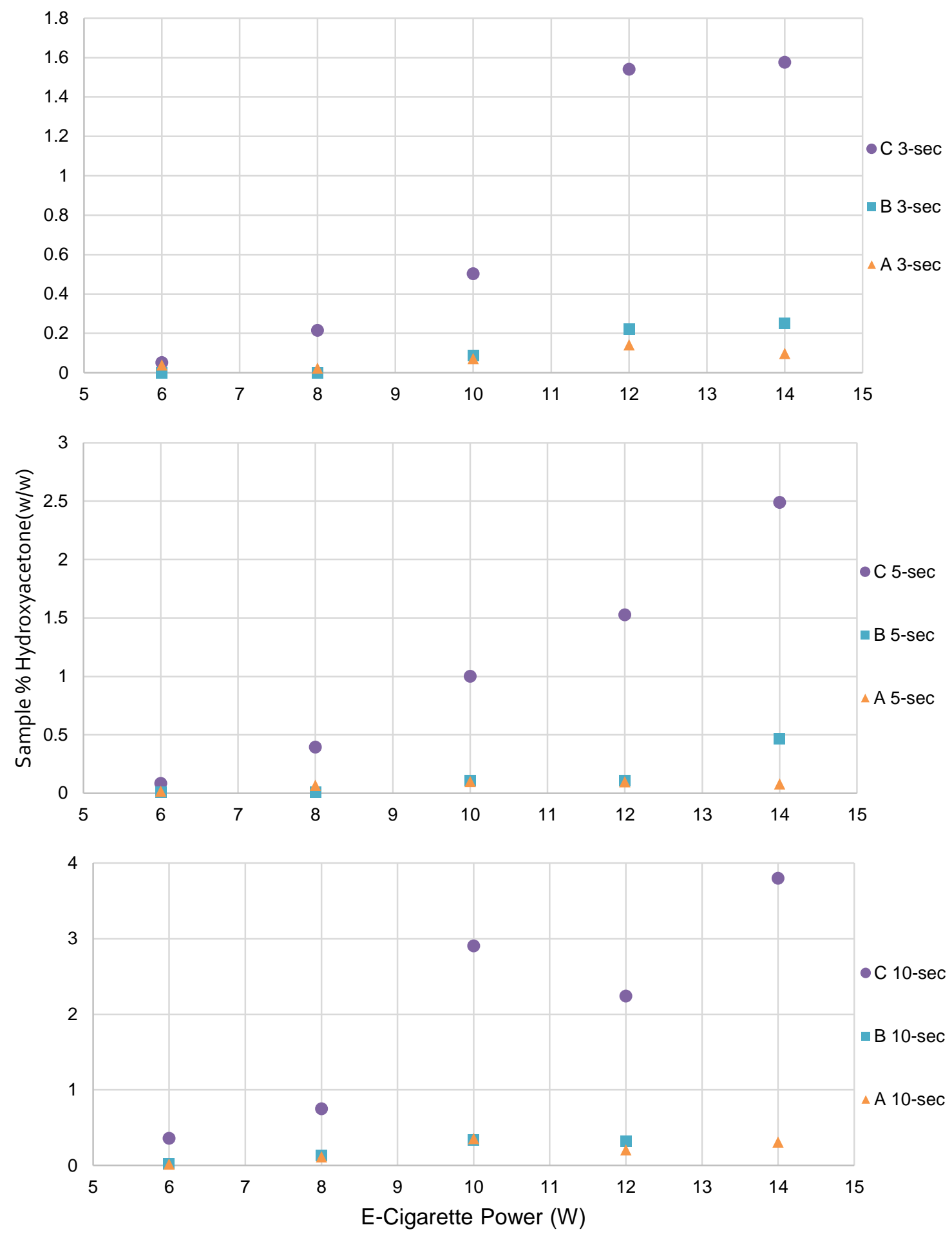

Figure 3.38 - Hydroxyacetone Values From Each Device, Separated by Puff Duration. 
Figure 3.38 shows that in each of the three conditions of puff duration $(3,5$, and 10 seconds), the samples vaporized using e-cigarette device $C$ contain significantly more hydroxyacetone than the samples collected from the other two devices, especially at high power levels. The extremely high levels of decomposition observed in samples vaporized using device $C$ is the source of the large error terms observed in Table 3.2 and Figure 3.37. The mass of (PG/GL) consumed from the reservoir of device $C$ during collection of the samples presented above is unremarkable in comparison with the other devices, indicating that there has been no failure to keep the wick and coil wetted with liquid during sample collection.

\subsubsection{Acetaldehyde}

Table 3.3 shows the concentrations of acetaldehyde in collected samples as measured by relative integration against an internal standard, along with calculated values of the mass percent of acetaldehyde in the sample $+/$ - the standard error in the measurement, which are

plotted in Figure 3.39. The error terms are again large relative to the calculated sample percentage, reflecting a high degree of variability between devices used in this experiment - this can be observed in Figure 3.40, which presents the calculated \% acetaldehyde generated by each of the three devices $(A, B$, and $C)$ used in this experiment. 
Table 3.3 - Quantification of Acetaldehyde.

\begin{tabular}{|c|c|c|c|c|}
\hline \multirow{6}{*}{$\begin{array}{c}3 \\
\sec \end{array}$} & Power (W) & $\begin{array}{c}\text { Average } \\
\text { [acetaldehyde] }(\mathrm{mM})\end{array}$ & $\begin{array}{c}\text { Average } \% \\
\text { acetaldehyde }(w / w)\end{array}$ & error \\
\hline & 6 & 0.003 & 0.003 & 0.002 \\
\hline & 8 & 0.013 & 0.007 & 0.006 \\
\hline & 10 & 0.130 & 0.042 & 0.032 \\
\hline & 12 & 1.037 & 0.427 & 0.345 \\
\hline & 14 & 1.620 & 0.557 & 0.443 \\
\hline \multirow{6}{*}{$\begin{array}{c}5 \\
\sec \end{array}$} & Power (W) & $\begin{array}{c}\text { Average } \\
\text { [acetaldehyde] }(\mathrm{mM})\end{array}$ & $\begin{array}{c}\text { Average } \% \\
\text { acetaldehyde }(\mathrm{w} / \mathrm{w})\end{array}$ & error \\
\hline & 6 & 0.023 & 0.005 & 0.004 \\
\hline & 8 & 0.397 & 0.097 & 0.072 \\
\hline & 10 & 1.287 & 0.376 & 0.278 \\
\hline & 12 & 2.087 & 0.680 & 0.548 \\
\hline & 14 & 2.323 & 1.124 & 0.868 \\
\hline \multirow{6}{*}{$\begin{array}{l}10 \\
\text { sec }\end{array}$} & Power (W) & $\begin{array}{c}\text { Average } \\
\text { [acetaldehyde] }(\mathrm{mM})\end{array}$ & $\begin{array}{c}\text { Average } \% \\
\text { acetaldehyde }(w / w)\end{array}$ & error \\
\hline & 6 & 0.130 & 0.022 & 0.018 \\
\hline & 8 & 0.510 & 0.098 & 0.053 \\
\hline & 10 & 1.477 & 0.730 & 0.406 \\
\hline & 12 & 4.163 & 1.671 & 1.351 \\
\hline & 14 & 3.117 & 2.042 & 1.665 \\
\hline
\end{tabular}




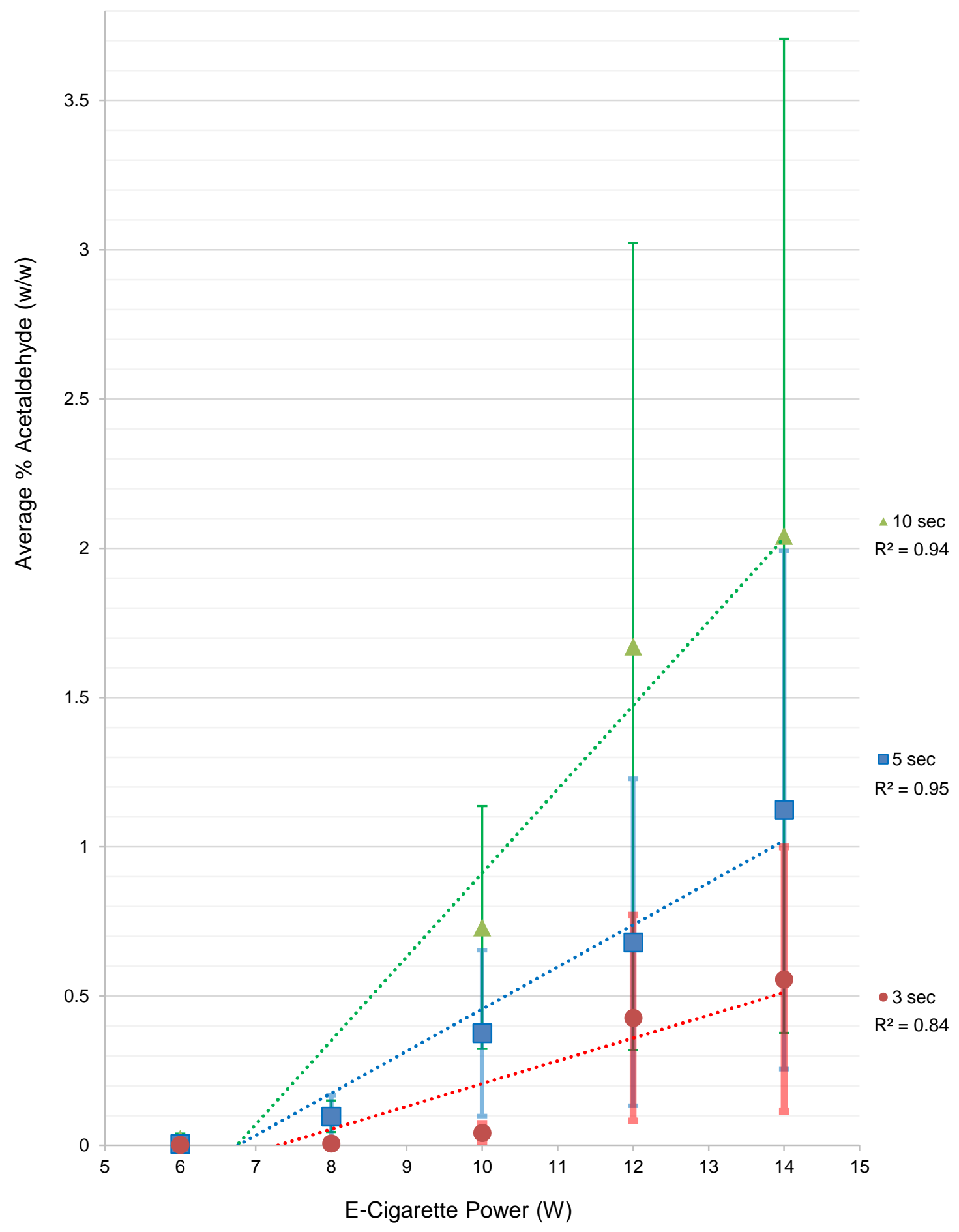

Figure 3.39 - Acetaldehyde Values Averaged from Three Devices. 

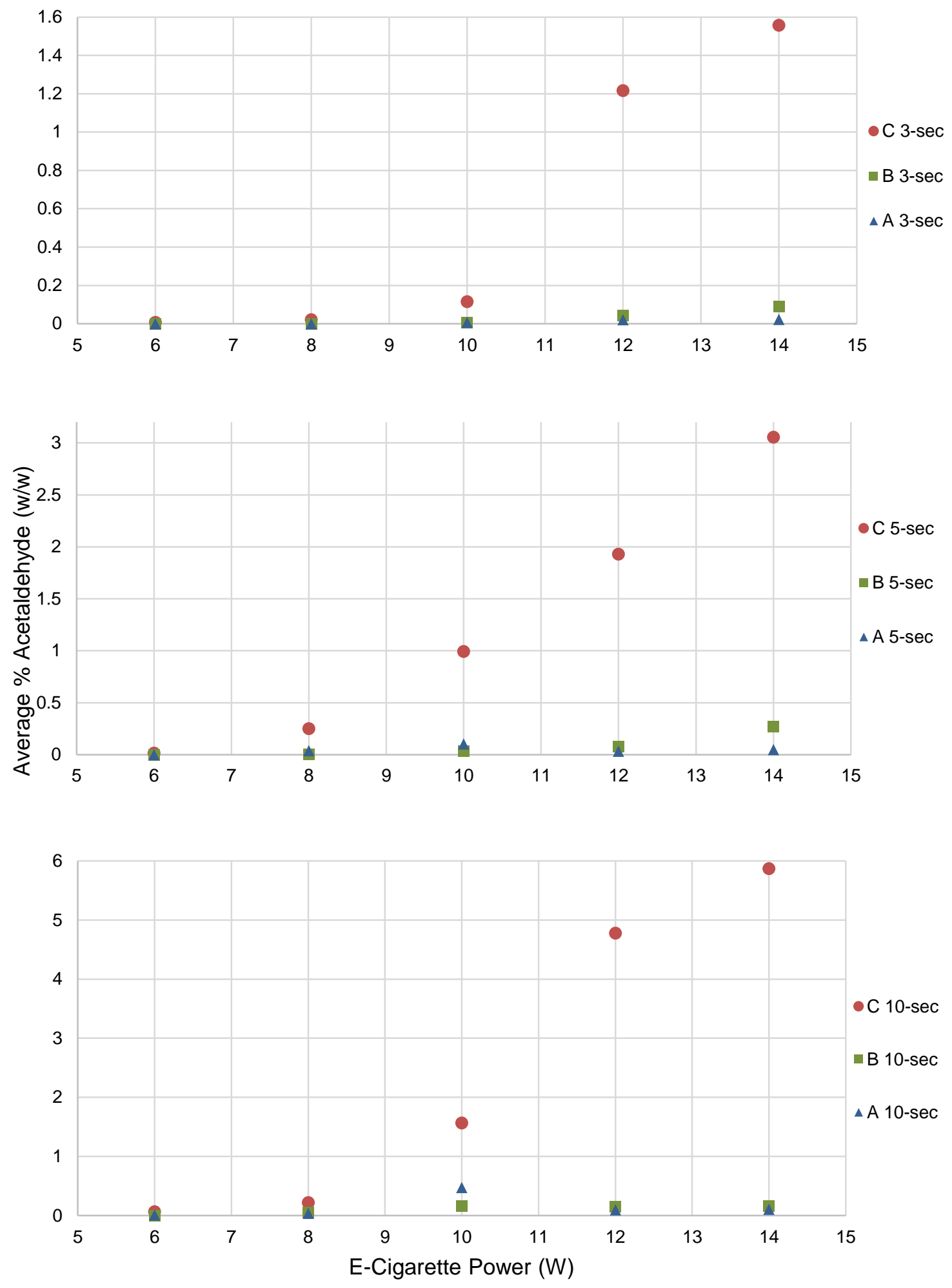

Figure 3.40 - Acetaldehyde Values From Each Device, Separated by Puff Duration. 
As was the case with hydroxyacetone, Figure 3.40 shows that in each of the three conditions of puff duration $(3,5$, and 10 seconds), the samples vaporized using e-cigarette device $C$ contain significantly more acetaldehyde than the samples collected from the other two devices. The extremely high levels of decomposition observed in samples vaporized using device $C$ is the source of the large error terms observed in Table 3.3 and Figure 3.39.

\subsubsection{Quantification Summary}

The difficulties encountered in attempting to precisely quantify the extent of decomposition in the samples discussed above reflect the variability between seemingly identical products. While the mass consumed during collection of the samples remained consistent across all three devices, the calculated mass percent of hydroxyacetone and acetaldehyde in samples from device $C$ were more than an order of magnitude higher than the values calculated for the samples collected from the other devices. Based on the results discussed above, it is tempting to conclude that the nature and condition of the heating element used to vaporize (PG/GL) is the most important factor in determining the extent of decomposition, significantly outweighing operating power and rate of air flow.

\subsection{Oxygen Dependence of Decomposition}

When measures are taken to limit the amount of oxygen present during collection of vaporized (PG/GL) samples, an obvious decrease in the intensity of many ${ }^{1} \mathrm{H}$ NMR peaks corresponding to decomposition products can be observed. Samples of vaporized (PG/GL) were collected in a sealed glove-bag that had been flushed with compressed nitrogen until a portable oxygen detector (BW Technologies GAXT-X-DL-2) indicated that $\mathrm{O}_{2}$ levels 
were $<0.1 \%$ of the atmosphere inside the bag. Anaerobic samples were collected in between aerobic samples as indicated in Table 3.4, which details the order the samples were collected in, the concentration of oxygen present in the glove-bag during collection of each sample, and the mass of each sample collected in the glass vial. The consumption of (PG/GL) was not possible to determine during this experiment because the cartomizer could not be removed from the glove-bag after each puff to determine its mass. The average of the six normalized ${ }^{1} \mathrm{H}$ NMR spectra from each condition are plotted in Figure 3.41 .

Table 3.4 - Conditions and Consumption of Aerobic/Anaerobic Samples.

\begin{tabular}{|c|c|c|}
\hline Sample \# & {$\left[\mathrm{O}_{2}\right](\%$ vol $)$} & Mass Collected $(\mathrm{mg})$ \\
\hline 1 & 20.9 & 30 \\
\hline 2 & 20.9 & 18 \\
\hline 3 & 20.9 & 25 \\
\hline 4 & $<0.1$ & 26 \\
\hline 5 & $<0.1$ & 34 \\
\hline 6 & $<0.1$ & 34 \\
\hline 7 & $<0.1$ & 42 \\
\hline 8 & $<0.1$ & 37 \\
\hline 9 & $<0.1$ & 31 \\
\hline 10 & 20.9 & 25 \\
\hline 11 & 20.9 & 14 \\
\hline 12 & 20.9 & 23 \\
\hline
\end{tabular}




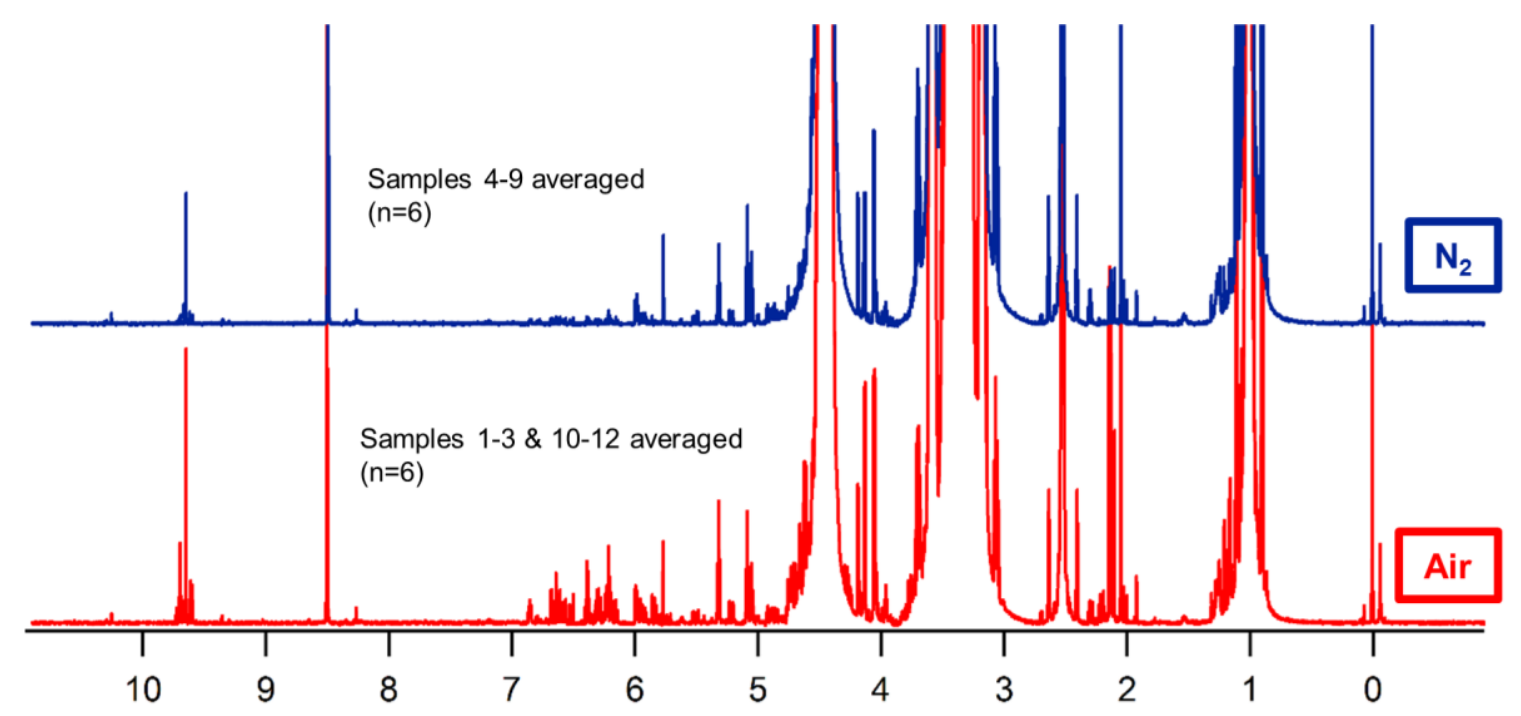

Figure 3.41 - Averaged ${ }^{1} \mathrm{H}$ Spectra (PG/GL) Vaporized Under Anaerobic and Aerobic Conditions.

The intensities of many peaks in the spectrum of the sample collected under nitrogen flow are significantly reduced when compared with samples collected under air flow, despite the fact that the samples collected under nitrogen are generally heavier than those collected under air. Some of the peaks associated with decomposition products in the anaerobic spectra, including glycolaldehyde and hydroxyacetone, appear to be less influenced by the absence of oxygen than others. These two species were observed to be the most abundant decomposition products in the anaerobic samples detailed in Table 3.4 - in fact, they were the only two decomposition products which were present in all twelve samples collected during both conditions of this experiment. Thus, these species were quantified in all samples by relative integration against $1.0 \mathrm{mM}$ 1,2,4,5-tetrachloro-3-nitrobenzene as discussed in Section 3.5.1.1. The results of this quantification are presented in Figures 3.42 and 3.43 , which show each sample treated independently and averaged together by condition. 


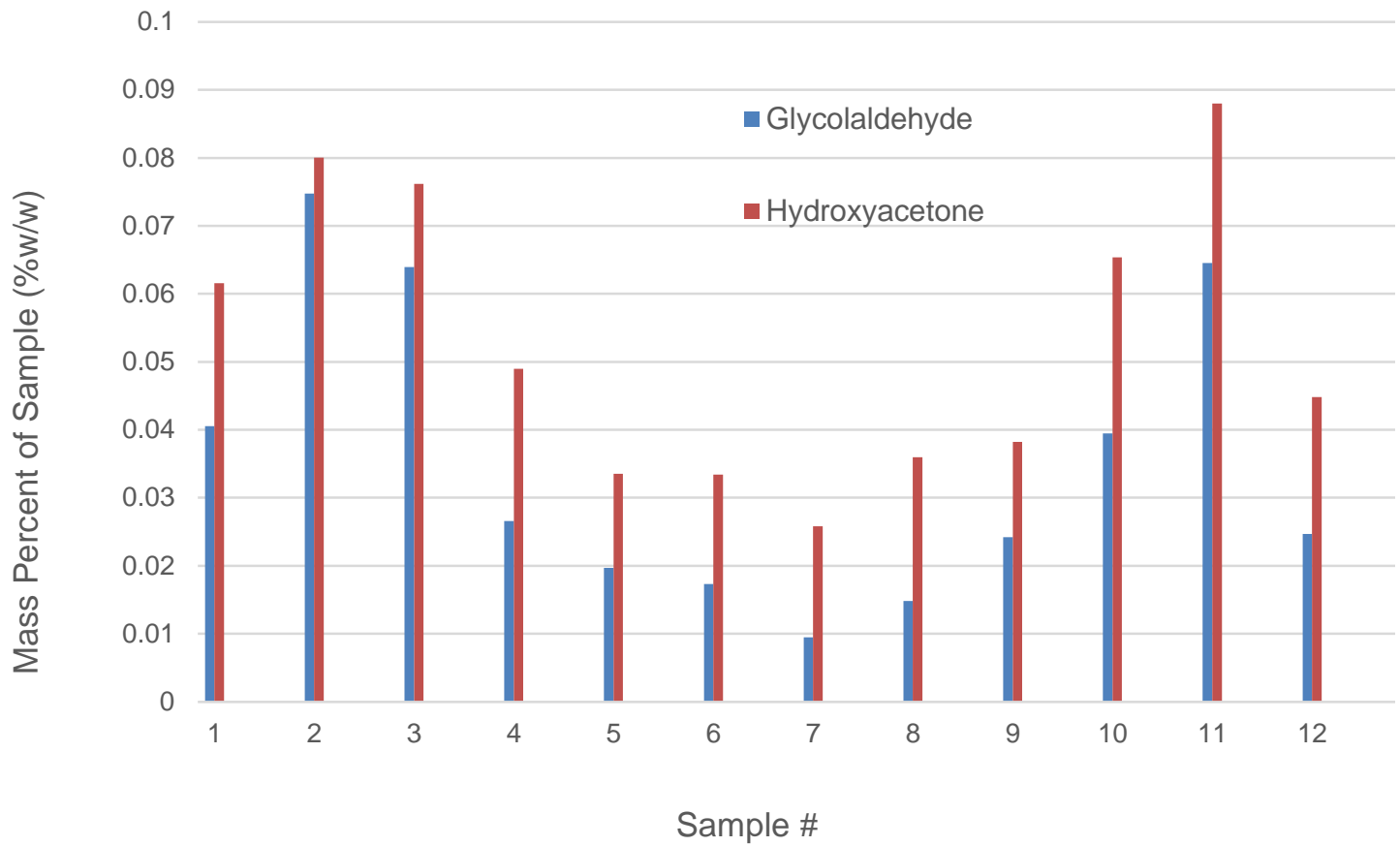

Figure 3.42 - Quantification of Hydroxyacetone and Glycolaldehyde in Anaerobic and Aerobic Samples.

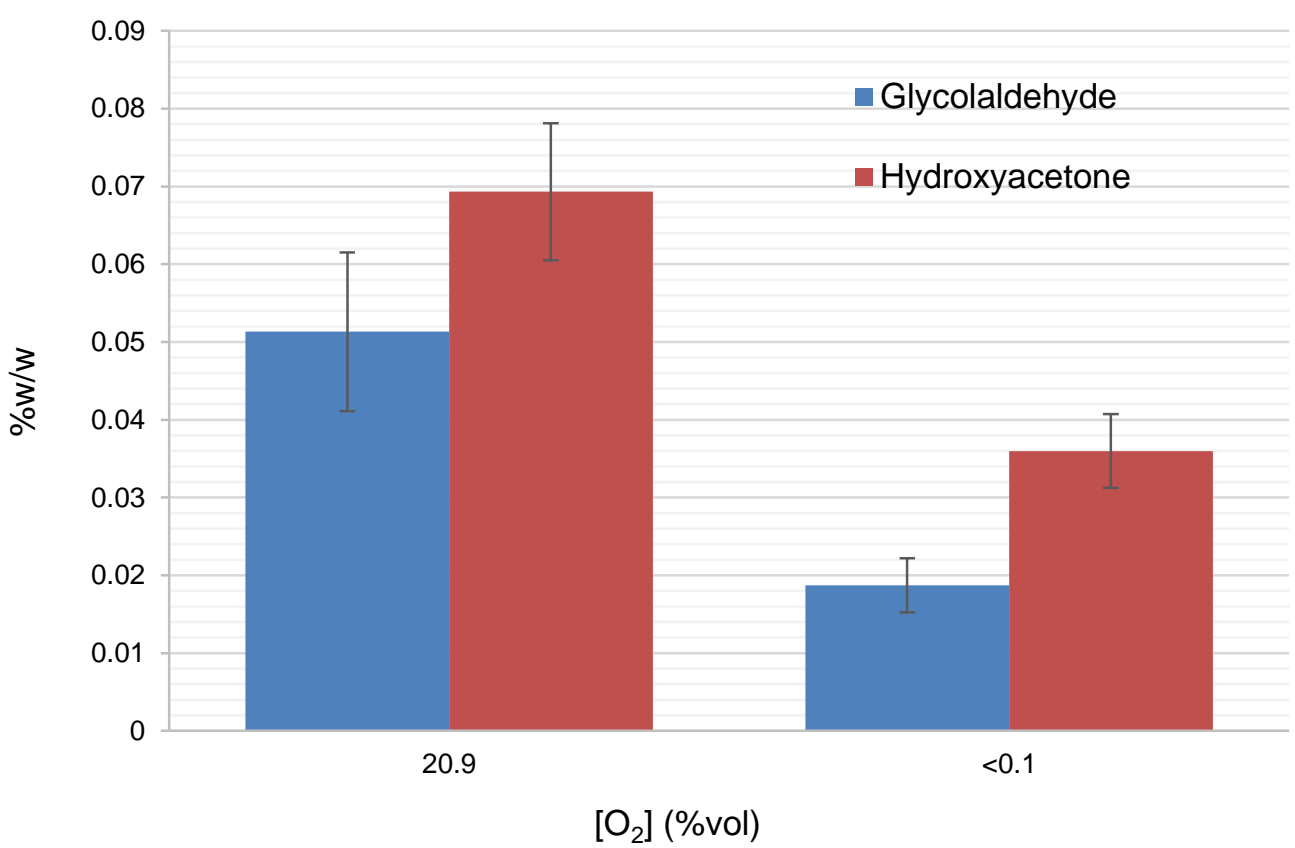

Figure 3.43 - Averaged Values of Hydroxyacetone and Glycolaldehyde in Anaerobic and Aerobic Samples. 
Considering that glycolaldehyde and hydroxyacetone are the decomposition products that remained most abundant in the anaerobic samples, these results indicate that removal of oxygen from the system has the general effect of decreasing the amount of decomposition in vaporized samples of (PG/GL). Similar treatment of other decomposition products such as acrolein, acetaldehyde, or allyl alcohol, which were not present at detectable levels in several of the anaerobic samples, would demonstrate a more striking but less generalizable contrast.

\subsection{Summary}

The major components of e-liquid - propylene glycol and glycerol - are susceptible to thermal decomposition under the conditions that they are vaporized using an electronic cigarette. It is possible to vaporize (PG/GL) without any evidence of decomposition, but when using the devices employed in this investigation, this is only typical at very low power levels. A large number of decomposition products have been identified and confirmed in samples of vaporized (PG/GL) analyzed by ${ }^{1} \mathrm{H}$ NMR spectroscopy. While (PG/GL) degradation tends to increase with increased e-cigarette operating power and increased puff duration, some catalytic aspect of the heating coil surface may be the most important factor in determining the extent and direction of thermal decomposition. The addition of nicotine and organic acids to the e-liquid mixture may complicate the chemistry of (PG/GL) decomposition - they would certainly complicate the analysis. Oxygen is very 
likely a reactant in many of the decomposition pathways discussed, and the presence of oxygen in the system may affect pathways in which it is not required in excess. 


\section{Formaldehyde}

Formaldehyde is unique, even among aldehydes. This small molecule, systematically referred to as methanal, is the first member of the aldehyde group and is extremely volatile in its molecular form, vaporizing at $-19^{\circ} \mathrm{C}$ under atmospheric pressure. The structure of this simple molecular form is shown in Figure 4.1, but this form is relatively unstable and reactive, making it difficult to isolate and handle. This type of formaldehyde is sometimes referred to as monomeric formaldehyde or carbonyl formaldehyde.

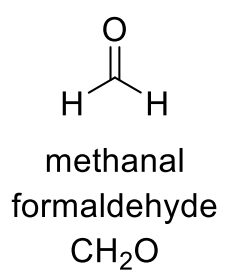

Figure 4.1 - Molecular Structure of Formaldehyde.

\subsection{Practical Characteristics of Formaldehyde}

Formaldehyde is rarely encountered in its simple molecular form - it exists more commonly as a linear polymer, a cyclic trimer, an aqueous hydrate, or as an acetal or hemiacetal adduct of an alcohol (or an aminal or hemiaminal of an amine). ${ }^{103}$ As discussed in Chapter 1, Section 1.1.3.2, an acetal of formaldehyde is known as a formal group. As such, a hemiacetal of formaldehyde is known as a hemiformal, discussed in detail in Section 4.6. These typical physical forms of formaldehyde are summarized in Scheme 4.1. 
$\underline{\text { meta-formaldehyde or } 1.3 .5 \text {-trioxane }}$ (cyclic trimer)

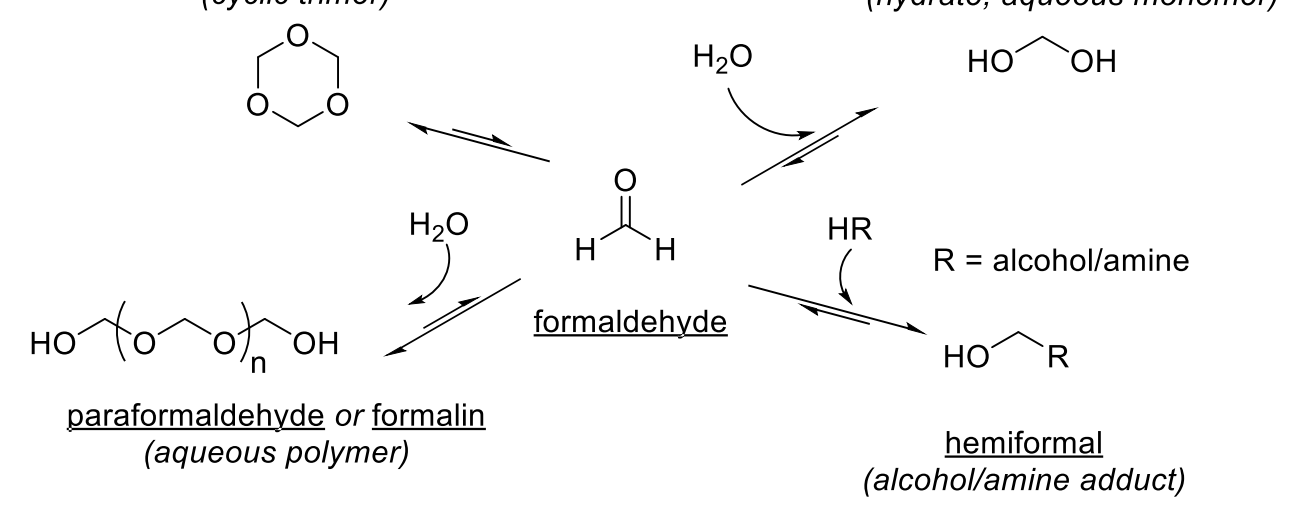

Scheme 4.1 - Stable Forms of Concentrated Formaldehyde.

Each of these intermolecular associations is a simple equilibrium and is subject to Le Châtelier's principle; solutions of formaldehyde tend to contain very little of the monomer, and the length and diversity of paraformaldehyde chains tend to grow as aqueous or alcoholic formaldehyde solutions become concentrated. ${ }^{\mathrm{XIV}}$ This chemical behavior and reactivity is not typical of other aldehydes - hemiacetals are generally regarded as unstable entities that exist transiently before the terminal condensation step of acetal formation.

\subsection{Formaldehyde Decomposition}

Formaldehyde can dissociate into carbon monoxide and hydrogen under pyrolysis conditions, ${ }^{104,105}$ and can oxidize further into carbon dioxide and water under combustion conditions, ${ }^{106}$ making it difficult to isolate from high temperature decomposition experiments. These decomposition routes are summarized in Scheme 4.2.

XIV Aqueous formalin solution may contain up to $55 \% \mathrm{w} / \mathrm{w}$ formaldehyde 


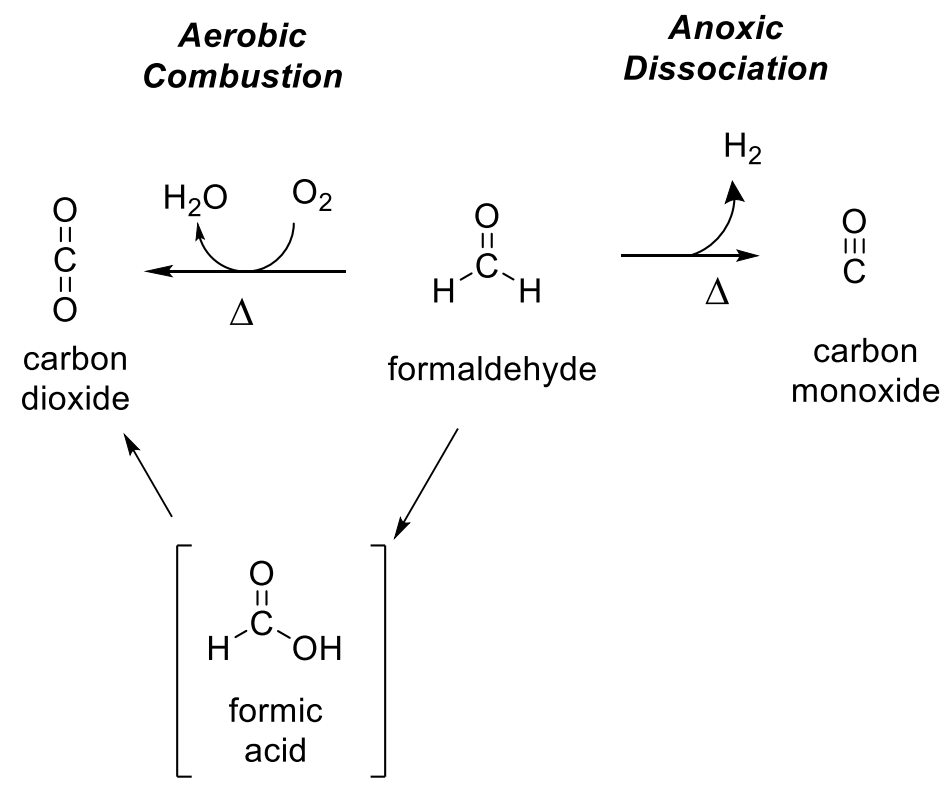

Scheme 4.2 - Thermal Decomposition Routes of Formaldehyde in Aerobic and Anoxic Systems.

\subsection{Initial Discovery of Formaldehyde}

Formaldehyde remained a theorized yet undiscovered compound until it was reported in 1859 by Alexander Mikhailovich Butlerov in an article about iodomethylene derivatives published in Annalen der Chemie. ${ }^{107}$ In this article Butlerov described an attempted synthesis of methylglycol (methylene glycol), the simplest possible glycol, which was noticeably missing from the glycol series published by Wurtz during that same year. ${ }^{43}$ Paralleling the glycol syntheses of Wurtz, he used silver acetate and a di-halogenated precursor to form a diacetate intermediate, in this case methylene diacetate, as shown in Scheme 4.3. 


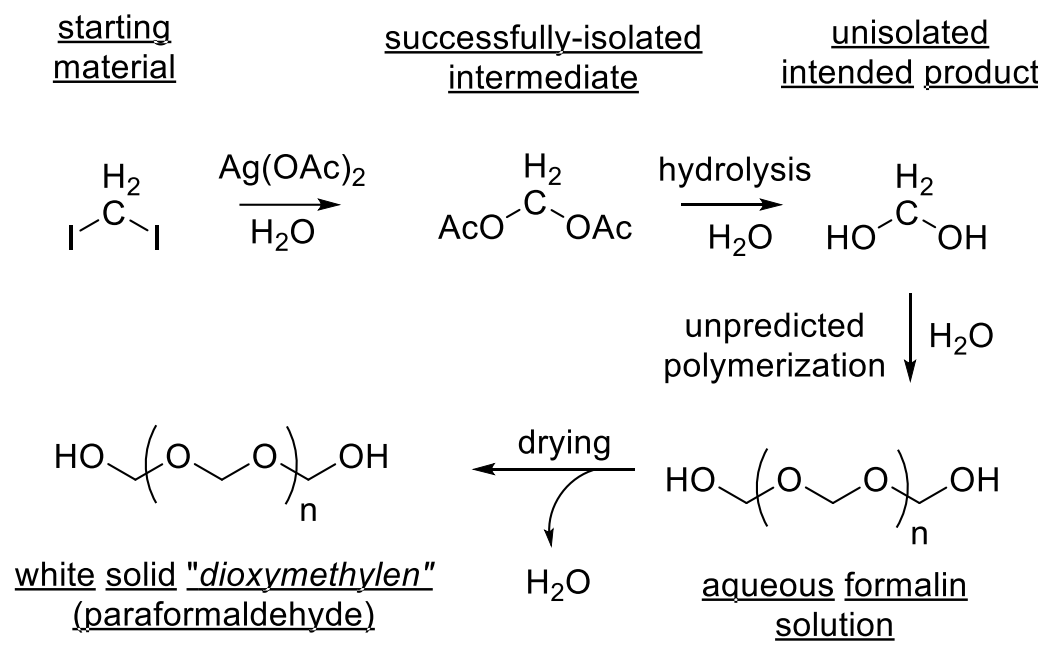

Scheme 4.3 - Butlerov's Failed Synthesis of Methylglycol or Butlerov's Accidental Synthesis of Paraformaldehyde.

Unfortunately, Butlerov found that the Wurtz method of acetate hydrolysis did not lead to simple methylglycol formation as it had for the heavier glycols, and he was unable to isolate methylene glycol in pure form. He found that heating the compound in a sealed glass tube filled with water eventually lead to the formation of acetic acid and a new compound that was isolated as a white solid upon evaporation of the water. This process is summarized in Scheme 4.3. Butlerov described this water-soluble compound as having acidic properties and stated that it partially volatilized when heated, forming a crystalline sublimate. $\mathrm{He}$ called the compound Dioxymethylen and wrote that one might consider the compound a true Methylglycoläther, which he referred to as a polymer of Formyl-aldehyd.

Butlerov did not know for certain that he was working with a polymer of formaldehyde not knowing how volatile the monomer is, he did not recognize that the vaporization process he observed upon heating Dioxymethylen was depolymerization - but he 
recognized the molecule by its reactivity despite his inability to isolate it or observe it in pure form. That accomplishment came less than a decade later in 1868 , when legendary German chemist and educator August Wilhelm von Hofmann reported he had identified the formaldehyde monomer by heating methanol with platinum in air. ${ }^{108}$

\subsection{The Formose Reaction}

In 1861, Butlerov published an article in Comptes Rendus entitled Formation synthetique d'une substance sucree, ${ }^{109}$ or "Synthetic formation of a sweet substance." In this article, Butlerov reported having boiled aqueous dioxyméthylène with calcium hydroxide, and after evaporating away the water he had observed the formation of crystalline calcium formate and a sweet substance he named méthylénitane that was soluble in alcohol and would not crystallize. This reaction, known as the formose reaction, is now understood to involve the combination of two formaldehyde monomers into glycolaldehyde, which itself combines with another formaldehyde monomer to form glyceraldehyde, resulting in a three-carbon sugar by the combination of three one-carbon molecules, as shown in Scheme 4.4. ${ }^{110}$ The reaction builds diverse mixtures of simple sugars under modest conditions in a process that is thought to proceed auto-catalytically. ${ }^{111}$ The formose reaction, wherein formaldehyde spontaneously polymerizes into sugars, is a fantastic example of the rather unusual chemistry in which formaldehyde participates. 


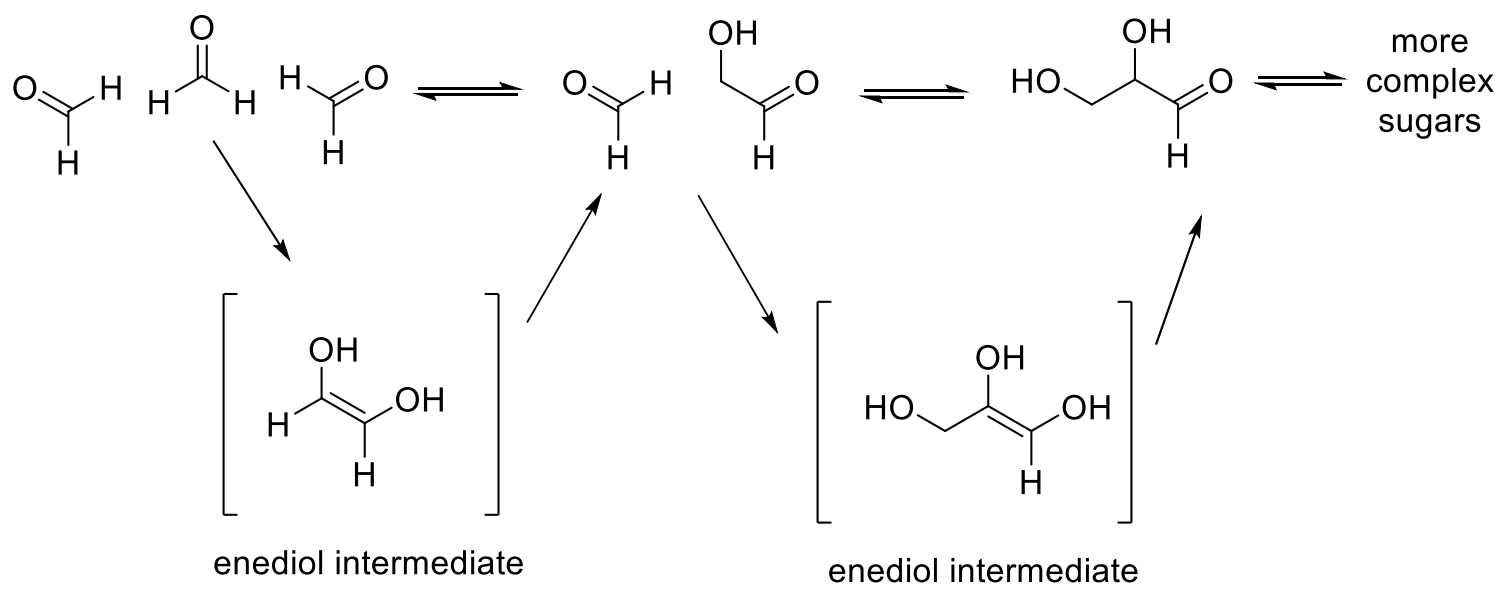

Scheme 4.4 - A Simplified Representation of the Initial Steps of the Formose Reaction.

\subsection{Formaldehyde as a Degradation Product of Glycerol and Propylene Glycol}

As was discussed in Chapter 1, Section 1.1.3, formaldehyde was identified as a degradation product of glycerol at $450{ }^{\circ} \mathrm{C}$ by John Nef in $1904 .{ }^{44}$ After fractionating the products of this decomposition, Nef noted that all of his distillation fractions smelled of formaldehyde, and a layer of meta-formaldehyde remained behind, coating his glassware. Nef identified formaldehyde and acetaldehyde as secondary degradation products of glycerol, being retroaldol dissociation products of the primary dehydration product hydroxyacetone. The only two gases that Nef identified as products of glycerol decomposition were carbon monoxide and hydrogen, which are the products of formaldehyde decomposition. In their often-cited 1983 article on glycerol pyrolysis, Stein and Antal did not detect sufficient formaldehyde to warrant quantification under their experimental conditions of $650-700{ }^{\circ} \mathrm{C}$ using FTIR and GC for analysis ${ }^{69}$ The 2007 article on isotopically-labeled glycerol pyrolysis by Paine 
et al. from Philip Morris USA used GC/MS to show that formaldehyde originates only from the terminal carbons of the three-carbon chain of glycerol. ${ }^{74}$ Recent computational studies by Nimlos et al. and Laino et al. predict formaldehyde and vinyl alcohol formation from the pyrolysis of glycerol, and an analogous mechanism is evoked by Díaz et al. to explain the formation of formaldehyde and acetaldehyde observed in their study of the oxidative thermal degradation of propylene glycol. ${ }^{75,77,99}$ A more detailed discussion is found in Chapter 1, Section 1.2.1.2.

\subsubsection{Formaldehyde as a Degradation Product of Electronic Cigarette Liquids}

Formaldehyde was identified as a decomposition product of electronic cigarette liquids as early as 2007, when Paine et al. from Philip Morris USA reported its evolution from the $\alpha$ carbons of glycerol. ${ }^{74}$ With the exception of my own work, ${ }^{112}$ detection of formaldehyde in vaporized e-liquid is typically conducted using DNPH cartridges, wherein carbonyl formaldehyde forms an adduct with 2,4-dinitrophenylhydrazine before chromatographic separation and analysis. This topic is discussed in Chapter 2, Section 6.

\subsection{Hemiformal}

According to Walker's ACS monograph Formaldehyde, monomeric formaldehyde is stabilized and solubilized by alcohols including glycols and glycerol. Formaldehyde is unmatched among aldehydes in its propensity to form stable hemiacetals; compounds formed by combination of formaldehyde with alcohols are called hemiformals. This process is depicted in Scheme 4.5. The Italian chemists Contardi and Ciocca reported in 
1936 that hemiformals were the products of reactions between polyhydroxy compounds such as sugars and formaldehyde. ${ }^{103}$

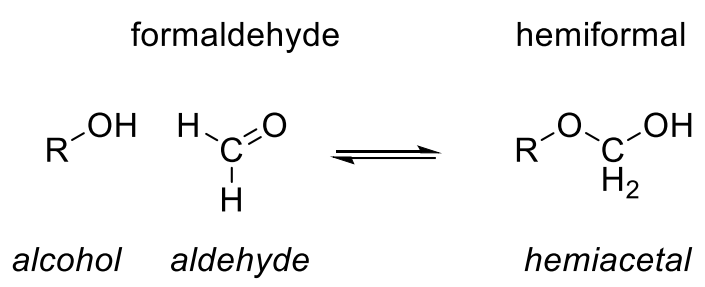

Scheme 4.5 - Hemiacetal Formation by Addition of Formaldehyde to an Alcohol.

Hemiformals are produced by reversible reactions and can be regarded as mixtures of formaldehyde and alcohols. Pyrolysis of hemiformals may be performed to generate pure monomeric formaldehyde. The simplest (and probably most common) hemiformal compound is methoxymethanol - shown in Scheme 4.6 - a compound that is occasionally referred to as hemiformal.

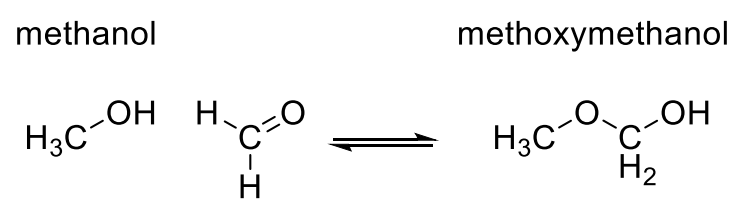

Scheme 4.6 - Reversible Formation of Hemiformal Compound Methoxymethanol.

Aqueous formalin solutions typically include a small percentage of methanol as a stabilizing agent, and the formation of methoxymethanol is generally accepted as the mechanism of stabilization - formaldehyde associates more favorably with alcohols than 
with water. Methoxymethanol was observed in 1983 by Silverman and Freeman from the Monsanto Company in St. Louis, Missouri using FTIR spectroscopy, ${ }^{113}$ and was confirmed by FTIR and GC/MS in 1991 by Johnson and Stanley from the Weapons Sciences division of the U.S. Army Missile Command in Huntsville, Alabama. ${ }^{114}$ Johnson filed a patent in 1990 for the GC/FTIR system that he designed for the detection of methoxymethanol. ${ }^{115}$

Hemiformals can be generated simply by bubbling carbonyl formaldehyde through an alcohol solution using a gas diffuser, as depicted in Figure 4.2. Under a flowing inert carrier gas, such as nitrogen, solid paraformaldehyde is decomposed by heating to $>100{ }^{\circ} \mathrm{C}$, liberating free monomeric formaldehyde that is volatile and easily carried through the system. The flowing gas may also have the effect of concentrating the hemiformal mixture by driving off excess unreacted alcohol if it is sufficiently volatile. Using this method, I have been able to purify methoxymethanol to $\sim 90 \%$ for a short time before the sample adopts an equilibrium mixture of $\sim 60 \%$ methoxymethanol, with the remainder comprised of oxymethylene dimers and trimers of methanol. 


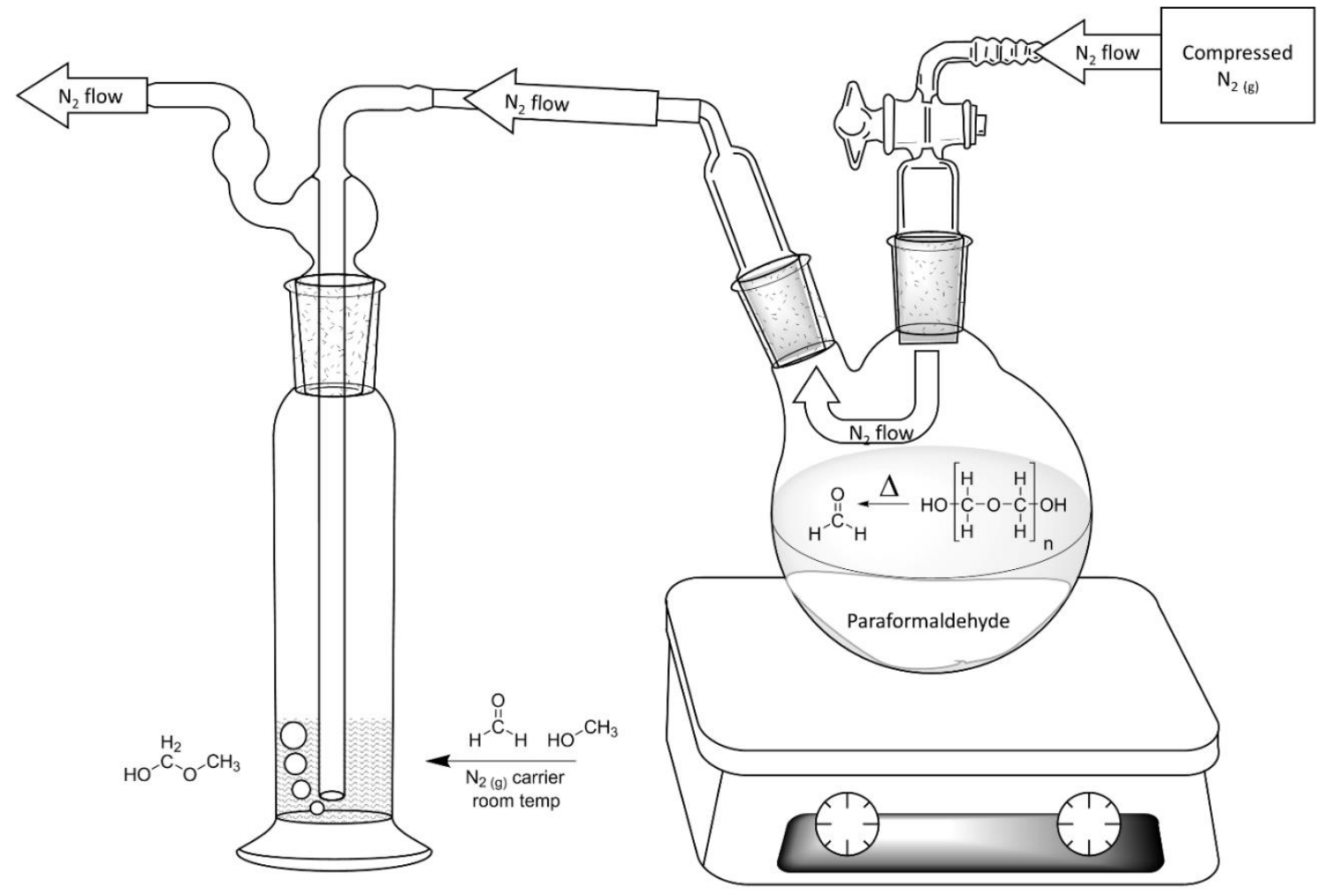

Figure 4.2 - Formaldehyde Bubbling Apparatus.

Hemiformals of propylene glycol and glycerol can be generated by this same method, but due to the low volatility of these compounds, flowing nitrogen gas is unable to enrich the sample by driving off excess alcohol. The high viscosity of glycerol makes it difficult to bubble gas through, but this problem can be mitigated by suspension in an appropriate volatile, aprotic solvent, such as acetonitrile. Unlike methanol, which has only one hydroxyl group and forms only one hemiformal (methoxymethanol), propylene glycol and glycerol each have multiple hydroxyl groups and can form a more complex ensemble of hemiformal adducts. Samples bubbled with only a small amount of formaldehyde tend to form dilute mixtures of mono-hemiformals, as shown in Scheme 4.7, where the $\alpha$-hydroxyl 
adducts are the major product (especially in the case of glycerol, which has two $\alpha$-hydroxyl groups). When large amounts of formaldehyde are reacted with propylene glycol or glycerol, complex mixtures of short poly(oxymethylene) adducts and di-hemiformals can also form.

propylene glycol mono-hemiformals

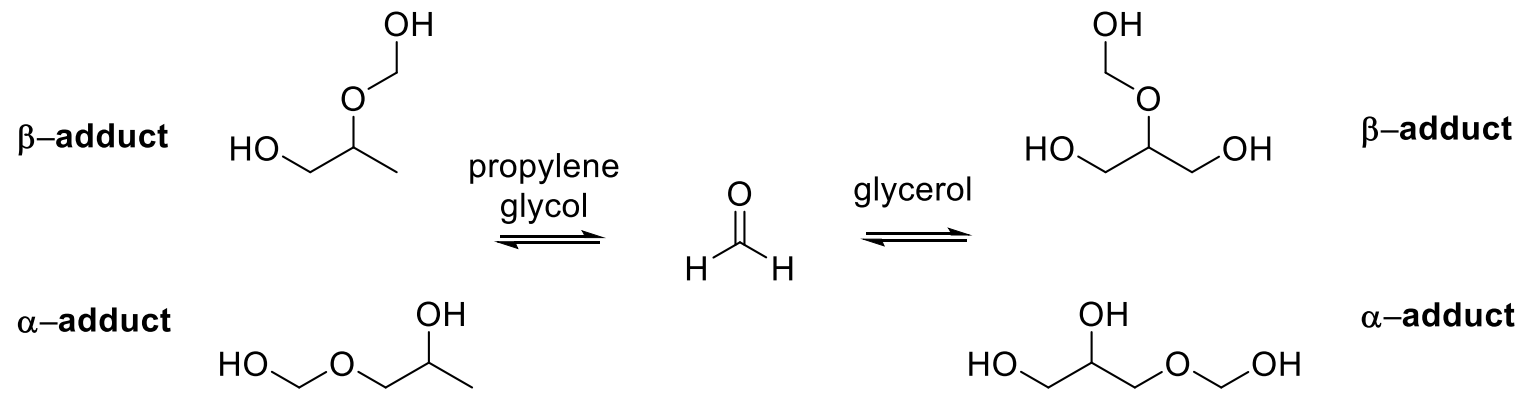

Scheme 4.7 - Glycerol and Propylene Glycol Mono-Hemiformals.

Glycerol and propylene glycol hemiformals release formaldehyde when they are dissolved in an alcohol or water, forming methylene glycol in aqueous solutions or a solvolysis product such as methoxymethanol when placed in an alcohol like methanol. This topic is covered in greater detail in Section 4.6.2.

\subsubsection{Hemiformal Detection by Nuclear Magnetic Resonance Spectroscopy}

Nuclear Magnetic Resonance spectroscopy of ${ }^{1} \mathrm{H}$ and ${ }^{13} \mathrm{C}$ allows the observation of hemiformals in solution. I have found that dilute samples of hemiformals such as 
methoxymethanol or glycerol $\alpha$-hemiformal, in an aprotic deuterated solvent such as DMSO-D 6 or DMF-D 7 , afford sharp ${ }^{1} \mathrm{H}$ resonances corresponding to the methylene and hydroxyl protons, which are coupled and can be observed by COSY analysis. The methylene carbon resonance can be observed by one-dimensional ${ }^{13} \mathrm{C}$ analysis and confirmed by ${ }^{1} \mathrm{H}_{-}{ }^{13} \mathrm{C}$ HSQC and HMBC analysis. Hydrogen-Deuterium Exchange (HDX) and Exchange spectroscopy (EXSY) experiments confirm that the hemiformal hydroxyl proton, which appears as a triplet at $6.2 \mathrm{ppm}$, is in exchange with the hydroxyl protons of water and methanol. These assignments were confirmed definitively using ${ }^{13} \mathrm{C}$-labeled paraformaldehyde to generate ${ }^{13} \mathrm{C}$-methoxymethanol by the method described in Figure 4.2. These experiments are summarized in Figure 4.3. The hemiacetal shown in the blue spectrum plotted in [A] was formed by bubbling ${ }^{13} \mathrm{C}$-formaldehyde through methanol. When a $\left\{{ }^{13} \mathrm{C}\right\}{ }^{1} \mathrm{H}$ decoupling pulse was used on this sample, as shown in the green spectrum in $[\mathrm{B}]$, one and two bond couplings $\left({ }^{1} J=160 \mathrm{~Hz},{ }^{2} J=4.0 \mathrm{~Hz}\right)$ are lost and the spectrum becomes identical to that of the hemiacetals formed by bubbling unlabeled formaldehyde through methanol, shown in red in [C], except for a small difference in the shift of the hydroxyl triplet caused by excess heat from the decoupling pulse. When this sample was titrated with $\mathrm{D}_{2} \mathrm{O}$, as shown in black in [D], the hydroxyl triplet at 6.2-6.1 ppm exchanges with bulk deuterium and the methylene doublet at 4.6-4.5 ppm loses three bond coupling $\left({ }^{3} J=7.8 \mathrm{~Hz}\right)$, becoming a singlet that shifts slightly with the changing composition of the solvent after $\mathrm{D}_{2} \mathrm{O}$ addition. This pattern of peaks is consistent across alcohols and is diagnostic of formaldehyde addition to a hydroxyl, as shown in [E] along with the full ${ }^{1} \mathrm{H}$ spectrum of methoxymethanol/methanol in DMSO-D 6 plotted in red on the bottom. 


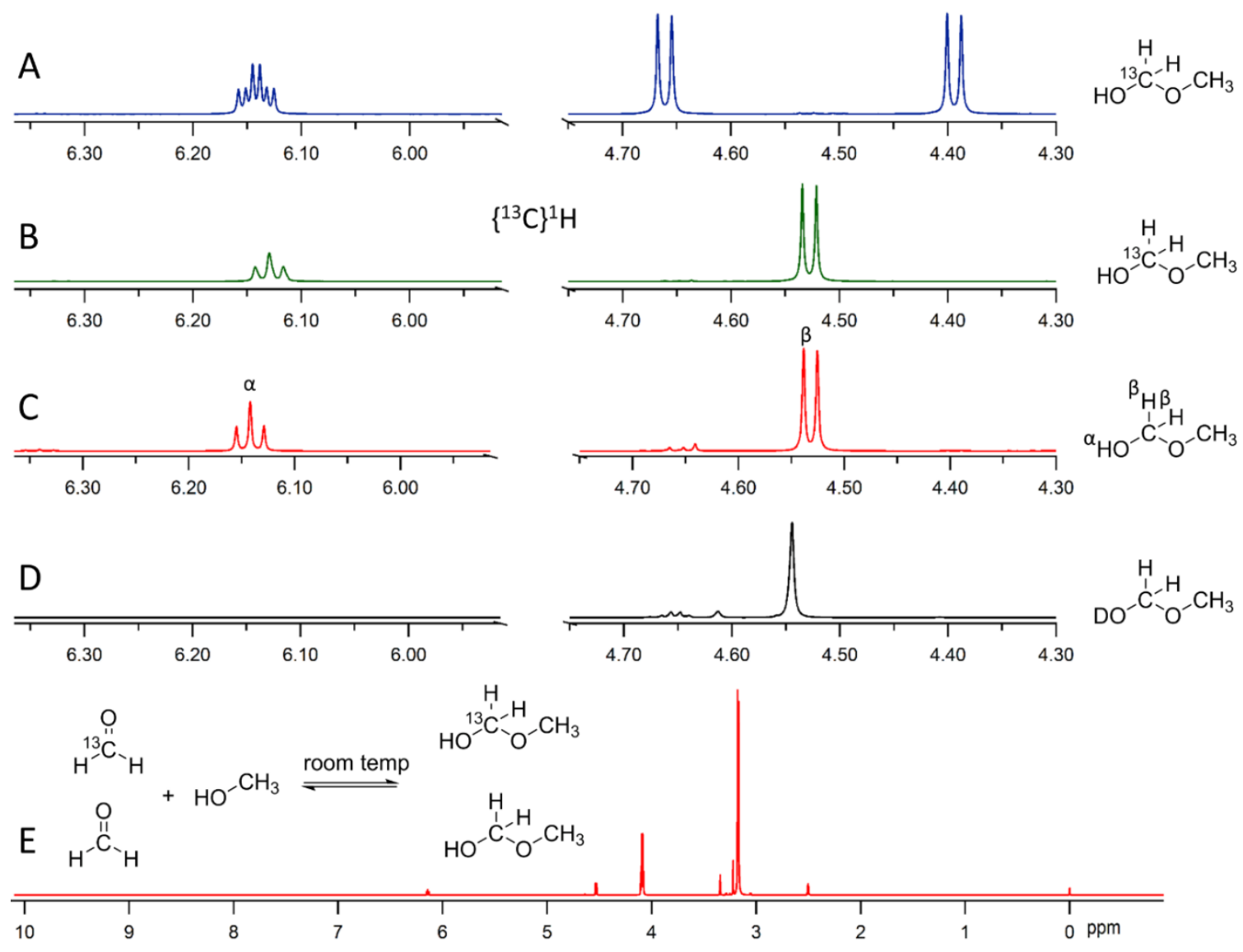

Figure 4.3 - Hemiformal Assignment and Confirmation by ${ }^{1} \mathrm{H}$ NMR Spectroscopy.

\subsubsection{Hemiformal Solvolysis}

As described in Schemes 4.1, 4.5, and 4.6, hemiformals are in a simple equilibrium relationship with carbonyl formaldehyde and an alcohol. When a hemiformal compound such as glycerol $\alpha$-hemiformal is diluted in water or an alcohol, the excess of solvent drives the solvolysis of the hemiformal, generating free glycerol and a formaldehyde-solvent adduct such as methylene glycol or methoxymethanol. This solvolysis can be studied by quantitative NMR spectroscopy, as in the experiment described below, in which 
methoxymethanol was added to $\mathrm{D}_{2} \mathrm{O}$, forming methylene glycol and methanol as shown in Scheme 4.8.

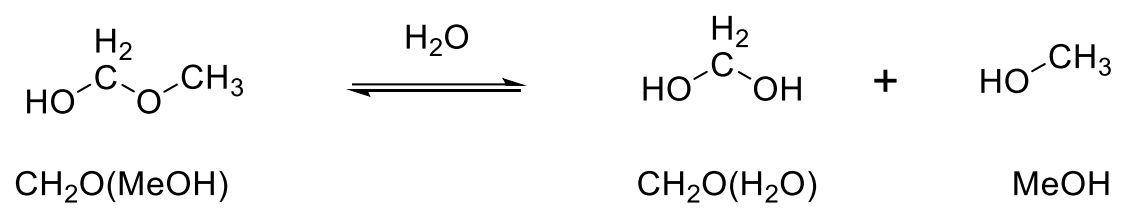

Scheme 4.8 - Hydrolysis of Methoxymethanol.

Concentrated methoxymethanol was prepared by bubbling carbonyl formaldehyde through methanol using the method described in Figure 4.2. The material was enriched to $\sim 90 \%$ pure (based on relative integration analyzed by ${ }^{1} \mathrm{H}$ NMR spectroscopy) and was used immediately after preparation. Nine samples of methoxymethanol were dissolved in deuterium oxide (99.9\%, Cambridge Isotope) at three different concentrations, each run in triplicate. These samples, detailed in Table 4.1, were allowed to equilibrate at room temperature for 12 hours before the equilibrium concentrations of the products and reactants were determined by ${ }^{1} \mathrm{H}$ NMR spectroscopy at $25{ }^{\circ} \mathrm{C}$ using relative integration. The ${ }^{1} \mathrm{H}$ NMR spectra of these samples are shown in Figure 4.4, plotting samples 1-3 in red, samples 4-6 in green, and 7-9 in blue. 
Table 4.1 - Initial Composition of Methoxymethanol Hydrolysis Samples.

\begin{tabular}{|c|}
\hline Sample \\
$\#$ \\
\hline 1 \\
2 \\
3 \\
\hline 4 \\
5 \\
6 \\
\hline 7 \\
8 \\
9 \\
\hline
\end{tabular}

\begin{tabular}{|ccc|}
\hline \multicolumn{3}{|c|}{ Volumes added to sample $(\mu \mathrm{L})$} \\
$\mathrm{CH}_{2} \mathrm{O}(\mathrm{MeOH})$ & $\mathrm{D}_{2} \mathrm{O}$ & Total \\
\hline 10 & 590 & 600 \\
10 & 590 & 600 \\
10 & 590 & 600 \\
\hline 20 & 580 & 600 \\
20 & 580 & 600 \\
20 & 580 & 600 \\
\hline 40 & 560 & 600 \\
40 & 560 & 600 \\
40 & 560 & 600 \\
\hline
\end{tabular}

\begin{tabular}{|cc|}
\hline \multicolumn{2}{|c|}{ Initial concentrations $(\mathrm{mol} / \mathrm{L})$} \\
{$\left[\mathrm{CH}_{2} \mathrm{O}(\mathrm{MeOH})\right]_{\mathrm{i}}$} & {$[\mathrm{D} 2 \mathrm{O}] \mathrm{i}$} \\
\hline 0.270 & 54.500 \\
0.270 & 54.500 \\
0.270 & 54.500 \\
\hline 0.539 & 53.576 \\
0.539 & 53.576 \\
0.539 & 53.576 \\
\hline 1.078 & 51.729 \\
1.078 & 51.729 \\
1.078 & 51.729 \\
\hline
\end{tabular}




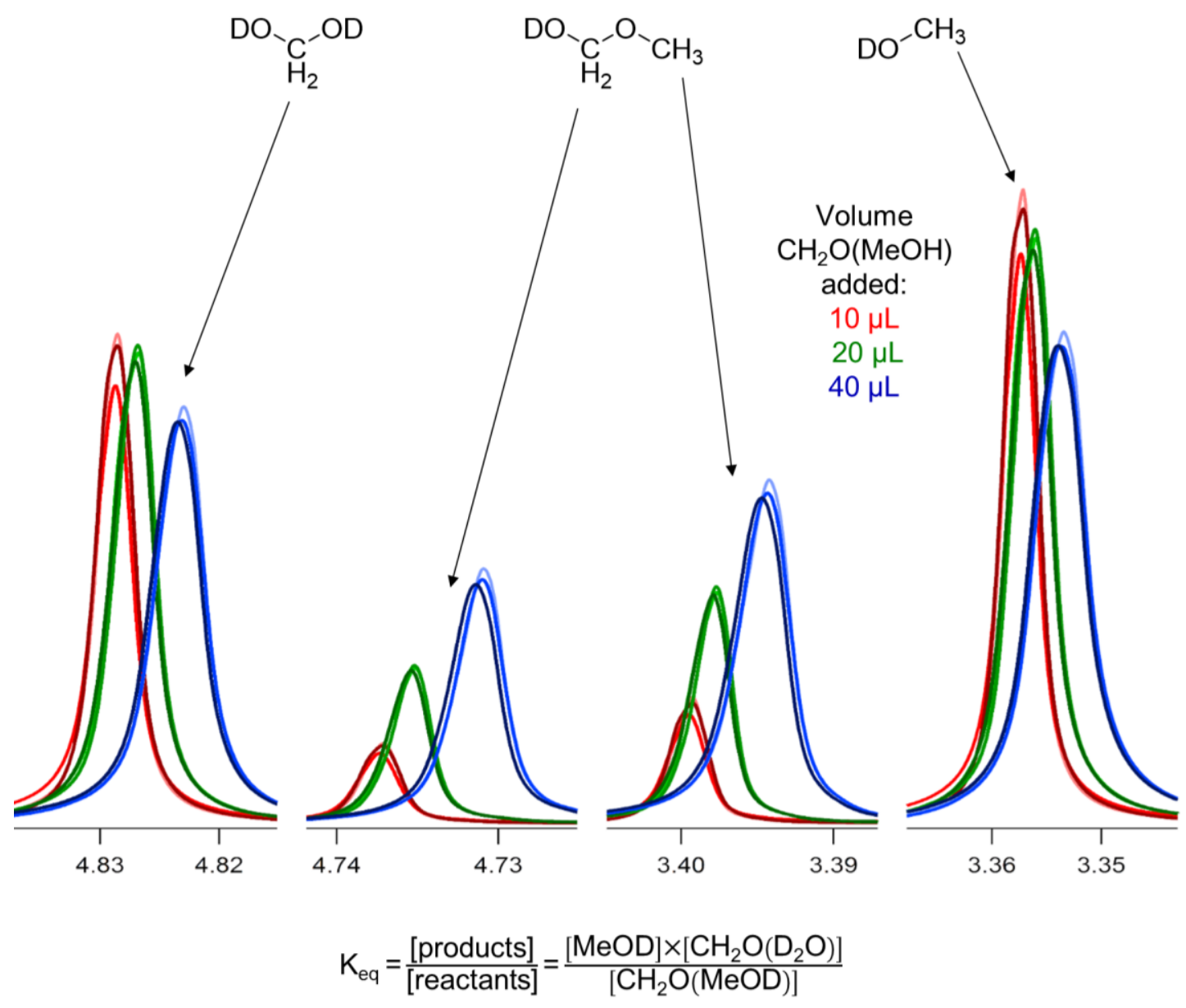

Figure 4.4 - Varied Concentrations of Methoxymethanol in $\mathrm{D}_{2} \mathrm{O}$ by ${ }^{1} \mathrm{H}$ NMR Spectroscopy at $25^{\circ} \mathrm{C}$.

The equilibrium concentrations determined from the spectra shown in Figure 4.4 were used to calculate the equilibrium constant $\left(\mathrm{K}_{\mathrm{eq}}\right)$ of methoxymethanol hydrolysis, as shown in Table 4.2. The calculated equilibrium constant is shown plotted with the ratio of methanol to methoxymethanol measured in each sample in Figure 4.5. 
Table 4.2 - Equilibrium Concentrations and $\mathrm{K}_{\mathrm{eq}}$ of Methoxymethanol Hydrolysis at $25^{\circ} \mathrm{C}$.

\begin{tabular}{|c|c|c|c|c|c|}
\hline $\begin{array}{c}\text { Sample } \\
\# \\
\end{array}$ & \multicolumn{3}{|c|}{ Equilibrium concentrations (mol/L) } & $\begin{array}{c}\text { Equilibrium } \\
\text { Constant } \\
\mathrm{K}_{\mathrm{eq}} \\
\end{array}$ & \\
\hline 1 & 0.266 & 0.035 & 0.235 & 1.80 & \\
\hline 2 & 0.260 & 0.036 & 0.234 & 1.73 & \\
\hline 3 & 0.266 & 0.035 & 0.235 & 1.80 & \\
\hline 4 & 0.467 & 0.112 & 0.427 & 1.81 & \\
\hline 5 & 0.470 & 0.113 & 0.426 & 1.81 & \\
\hline 6 & 0.468 & 0.115 & 0.424 & 1.77 & \\
\hline 7 & 0.784 & 0.346 & 0.732 & 1.72 & \\
\hline 8 & 0.786 & 0.350 & 0.729 & 1.70 & \\
\hline \multirow[t]{2}{*}{9} & 0.788 & 0.348 & 0.730 & 1.71 & \\
\hline & & & & $\begin{array}{l}1.76 \\
0.04 \\
2.48\end{array}$ & $\begin{array}{c}\text { Average } \\
\text { S.D. } \\
\text { \%rsd }\end{array}$ \\
\hline
\end{tabular}

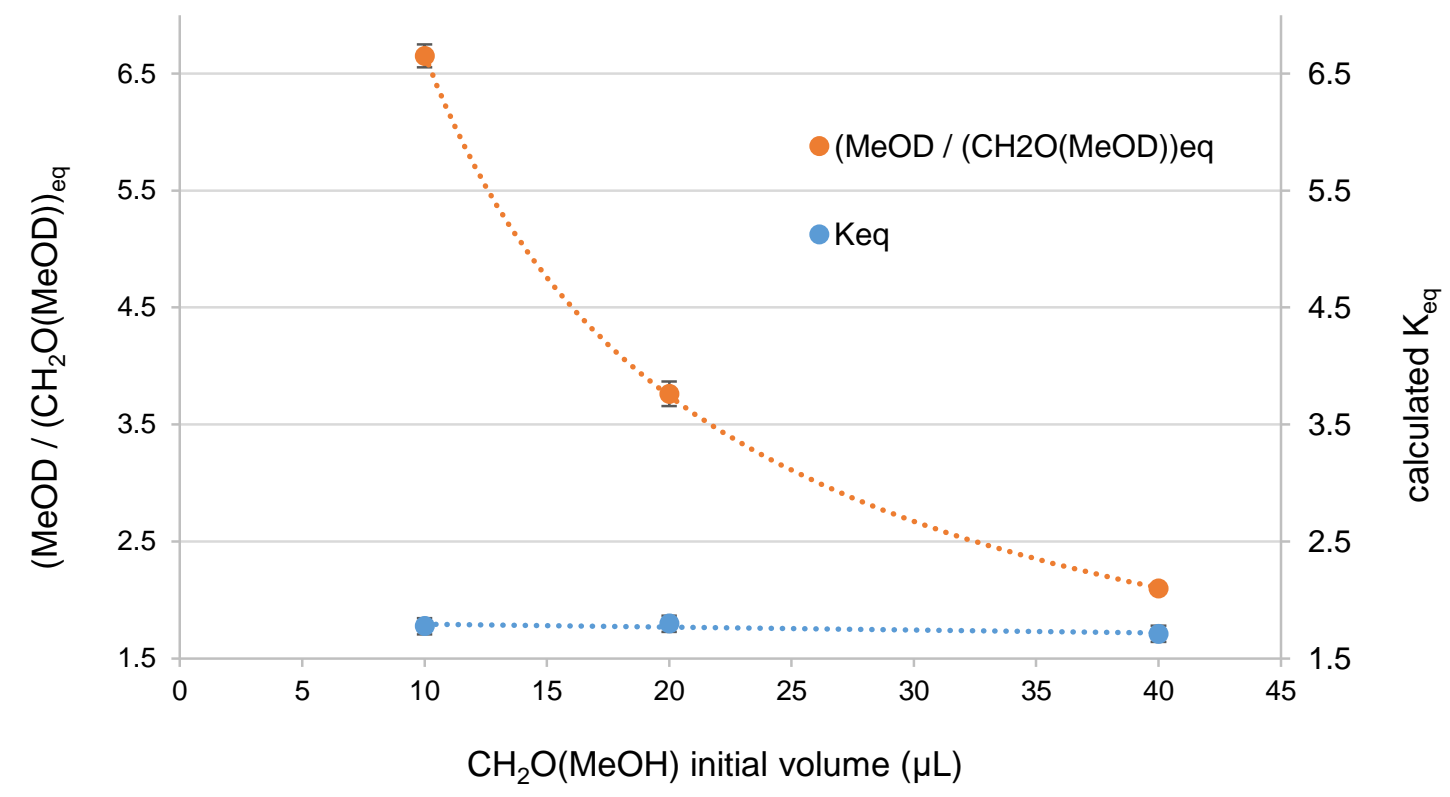

Figure 4.5 - Comparison of Measured Ratios and Calculated Equilibrium Constants. 
The equilibrium constant was similarly determined at variable temperatures, and the results were used to construct a van't Hoff plot, plotting the natural logarithm of the equilibrium constant on the vertical axis against the reciprocal of the temperature on the horizontal axis, as shown in Figure 4.6. Analysis of the van't Hoff plot in Figure 4.6 indicate that the hydrolysis of dilute methoxymethanol is an endothermic process that is driven by increased entropy and will be spontaneous at temperatures above $261 \mathrm{~K}$.

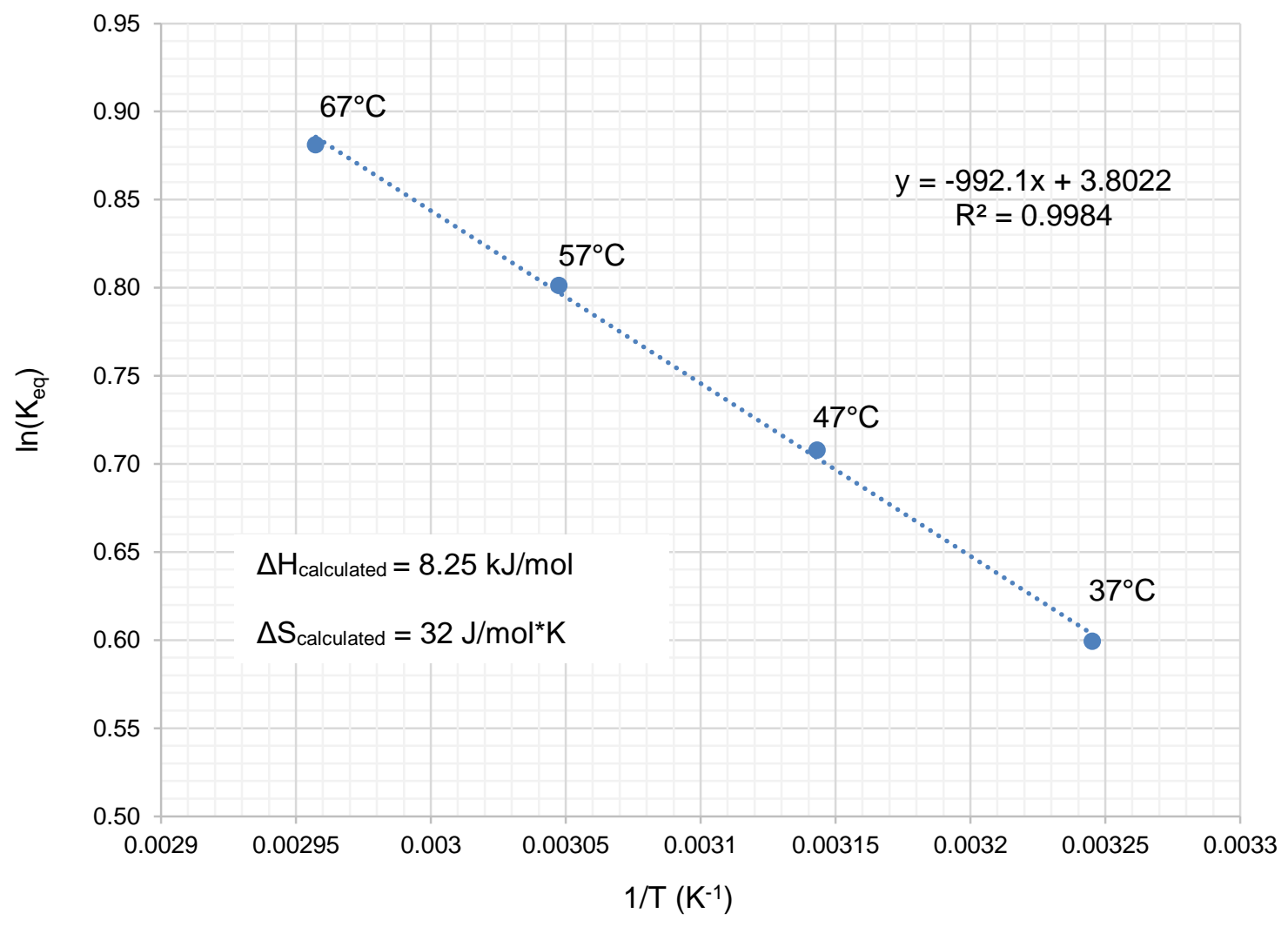

Figure 4.6 - Van’t Hoff Plot of Methoxymethanol Hydrolysis.

The slope-intercept form of the van't Hoff equation sets the natural logarithm of the equilibrium constant equal to the negative change in enthalpy divided by the value of the 
gas constant multiplied by temperature, plus the change in entropy divided by the gas constant. When the natural logarithm of experimentally determined equilibrium constants are plotted on the vertical axis of a Cartesian graph against the reciprocal of temperature on the horizontal axis, the change in enthalpy becomes equal to the slope of the resulting line multiplied by the negative gas constant, while the change in entropy is equal to the point at which the line intercepts the vertical axis multiplied by the gas constant.

\subsection{Summary}

Formaldehyde is a volatile and reactive molecule that has been known to arise from decomposition of glycerol since at least 1904, and was discussed as a decomposition product of an electronic cigarette as early as 2007 . The unusual reactivity of formaldehyde complicated its original discovery and makes it difficult to isolate as a monomer. Formaldehyde generated by decomposition at high temperatures may degrade further into carbon monoxide or carbon dioxide if heat is sufficient. Carbonyl formaldehyde spontaneously recombines into a number of different forms, and generates adducts with alcohols including propylene glycol and glycerol. These hemiformal adducts are stable and were detected in this work by ${ }^{1} \mathrm{H}$ NMR spectroscopy, which was used quantitatively to study the thermodynamics of their hydrolysis - a process that was found to be spontaneous. 


\section{Shisha}

Smoking tobacco out of a pneumatic water pipe - henceforth referred to as a hookah, though many alternative names exist - has been common practice in Western and Central Asia for hundreds of years. This practice has spread worldwide, expanding even into cultures that are conscious of the harmful effects of smoking. Recent data suggests that American teenagers are increasingly likely to have smoked hookah while the use of traditional forms of tobacco has declined among this demographic. ${ }^{116,117}$

\subsection{How Shisha is Smoked}

A hookah is simply a large pipe fitted with a bowl containing a sample of tobacco that is heated by burning charcoal. A vertical metal tube connects this bowl to a vessel partially filled with water, with a hose connecting the head-space of the vessel to a mouthpiece. Inhalation by a user draws air and smoke from the bowl of heated tobacco down the tube, where the smoke exits the water as bubbles and fills the head-space of the vessel before exiting through the hose into the user's respiratory tract. The large volume of the system requires the user to take longer, deeper breaths to draw in smoke as compared to smoking a handheld pipe or tobacco rolled in paper. The water filling the vessel through which the smoke bubbles are pulled is supposed to extract a fraction of the water-soluble components from the smoke before it reaches the user.

The tobacco that is smoked with a hookah is not limited to any specific kind or type. This technique was traditionally used to smoke dried tobacco leaves; however, the most 
common type of tobacco used in the modern era is a wet mixture of glycerol, honey or molasses, flavorants, and dried tobacco leaves. Mixed tobacco of this type goes by many names, but shisha is among the most common. In general, shisha contains only a small fraction of the nicotine that is found in other consumer tobacco products. The process of smoking a bowl of shisha using a hookah is deliberate and unhurried, and is intended to be relaxing whether done privately or socially. The relatively low nicotine content, large inhalation volume, and unique heating method make hookah smoking a long, slow process that is difficult to compare directly to other methods of tobacco use, although public health advocates have endeavored to make such a comparison.

\subsection{The Chemistry of Shisha}

From a chemical perspective, the type of shisha that is commonly found in American smoke shops is more similar to electronic cigarette liquids than to dried tobacco products. Depending on the product, the tobacco leaves themselves might make up less than $10 \%$ (w/w) of a sample of shisha, while glycerol may constitute upwards of $50 \%$ of the mass of a wet sample. Along with glycerol, detectable levels of propylene glycol or ethanol may be added to modulate the smoking characteristics or as delivery vehicles for flavorants. Flavorants that are commonly used in e-liquids, including vanillin and benzaldehyde, are also used in shisha. The presence of small sugar molecules, added to the mixture as honey or molasses, is a feature unique to shisha among tobacco products.

With large amounts of glycerol, flavorants, and sugars added to shisha, the smoke produced is described as "softer" on the respiratory system of the user, allowing for large puffs of 
flavorful smoke. The relatively low nicotine content allows users to take many large puffs without inhaling an excessive dose of the powerful stimulant. Some commercial shisha products are specifically marketed as being entirely free of nicotine, demonstrating the social and behavioral motivations of this technique, which are certainly not unique to hookah smoking.

\subsection{Smoking, Filtration, and Degradation}

A bowl of shisha is typically heated using charcoals, which smolder at several hundred degrees above room temperature. When considering the long, deep puffs of shisha smoke that may be taken from a hookah and the slow pace of a smoking session, this unique method of heating could potentially cause thermal decomposition of glycerol. Air is pulled past the burning coals into the bowl, mixing with the heated shisha before carrying aerosolized droplets of shisha smoke through the hookah and out through the mouthpiece. Some elements of shisha, especially glycerol and sugars, are susceptible to dehydration and oxidation at temperatures attained by the burning coals. Over time, the water within the vessel through which the shisha smoke is pulled takes on an odor and discoloration, suggesting that it has absorbed material from the passing smoke. The efficiency and selectivity of this filtration is unknown; once discoloration has occurred, the water is typically discarded and the vessel refilled.

In this work, the sample collection method described in Chapter 3, Section 2, which was developed to collect samples of vaporized electronic cigarette liquids as they exit the mouthpiece of the device, was adapted to collect samples of shisha smoke as they exit the 
mouthpiece of the hookah hose. Smoked shisha samples were collected using a $500 \mathrm{~mL}$ syringe as a vacuum source instead of the $50 \mathrm{~mL}$ syringe employed in the collection of vaporized e-liquid samples. The $1500 \mu \mathrm{L}$ glass vials used to collect vaporized e-liquid samples were changed to $4000 \mu \mathrm{L}$ vials, which could accommodate larger 10 gauge needles required to avoid an air flow constriction using the $500 \mathrm{~mL}$ syringe. Using this method, samples of shisha smoke were collected into DMSO-D 6 and analyzed by ${ }^{1} \mathrm{H}$ NMR spectroscopy.

\subsection{Smoked Shisha}

Samples of smoke from a commercially available shisha product (Starbuzz ${ }^{\circledR}$ Blue Mist) were collected into DMSO-D6 according to the method described in Chapter 3, Section 2, along with the amendments detailed above. Un-smoked shisha was extracted into DMSO$\mathrm{D}_{6}$ for comparison. The ${ }^{1} \mathrm{H}$ NMR spectra from samples of these types are presented in Figure 5.1, featuring smoked shisha plotted in red on top of unsmoked shisha plotted in black. A number of peaks associated with sugars from honey that appear in the unsmoked sample are absent from the smoked sample. The spectrum of the smoked sample shows evidence of decomposition, in the form of new peaks corresponding to degradation products of glycerol and sugars. These compounds include formic acid, glycidol, and 5hydroxymethylfurfural, which is a double-dehydration product of glucose. 


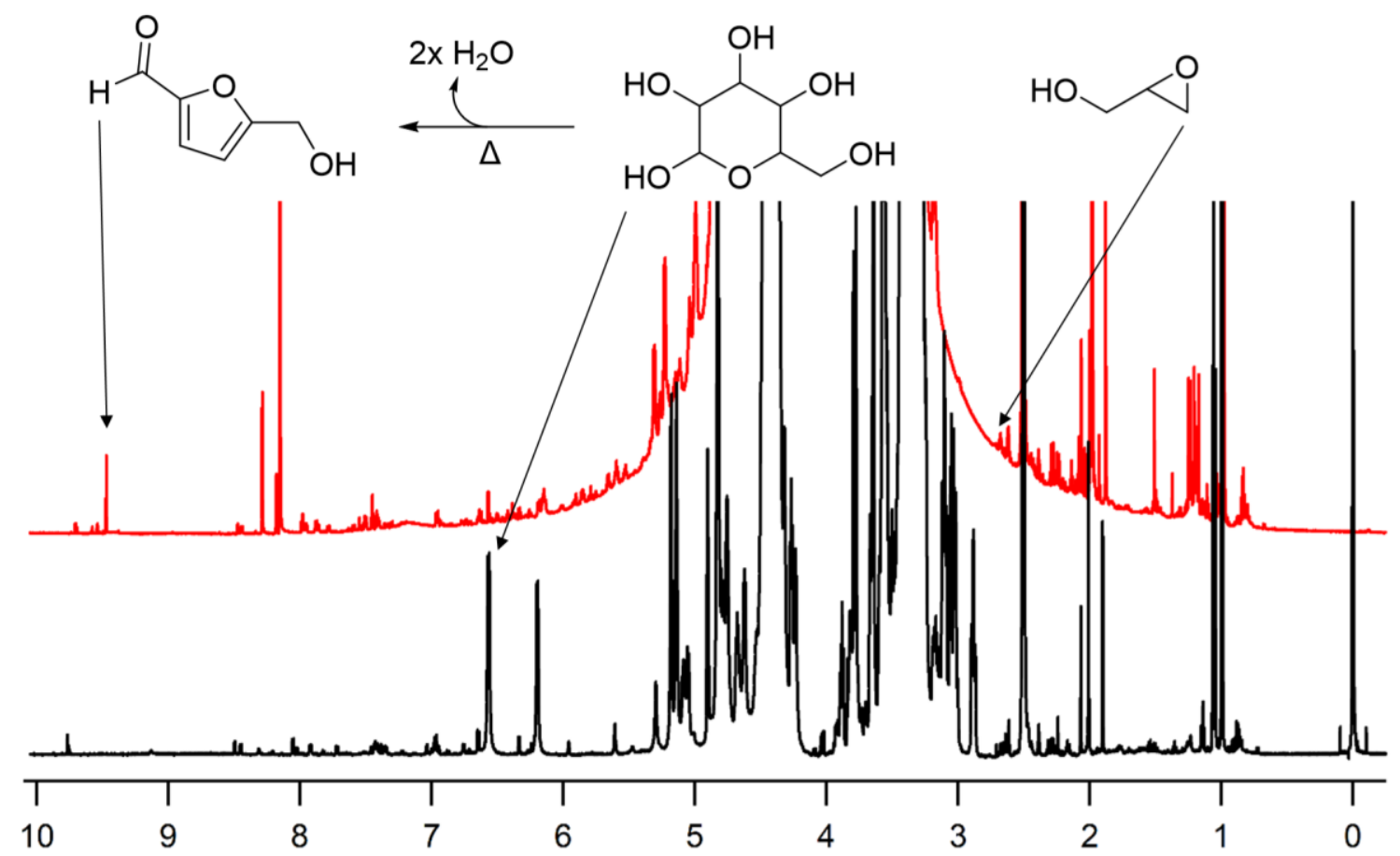

Figure 5.1 $-{ }^{1} \mathrm{H}$ NMR Spectra of Smoked (red) and Unsmoked (black) Shisha.

In Figure 5.1, plotted in black on bottom is a spectrum from an unsmoked shisha sample extracted into DMSO-D 6 . Plotted in red on top is a spectrum from a smoked shisha sample collected into DMSO-D 6 , using a variation of the method developed for sampling e-liquids described in Chapter 3, Section 2. The unsmoked shisha contains sugars and ethanol that are present to a much smaller extent in the smoked sample. The smoked sample contains peaks corresponding to decomposition products of glycerol, such as glycidol and formic acid, and well as decomposition products of honey - most notably, 5hydroxymethylfurfural. 


\subsection{Hookah Water}

The water filling the vessel of the hookah, through which shisha smoke is pulled, takes on color and odor over time and is supposed by users to provide some filtration. With this in mind, a very small vessel was developed that could be filled with a small volume of $\mathrm{D}_{2} \mathrm{O}$ through which shisha smoke was pulled. This smoke was collected into a sample vial containing $\mathrm{D}_{2} \mathrm{O}$ as described above, and the ${ }^{1} \mathrm{H}$ NMR spectrum of the smoke was compared to the spectrum of the water through which smoke had been pulled. These spectra are presented in Figure 5.2, in which the spectrum of hookah water through which shisha smoke was pulled is plotted in red across the bottom, while the spectrum of the shisha smoke itself is plotted in blue across the top. The spectra contain different profiles of peaks, corresponding to shisha components and decomposition products. Nicotine, which is present in the smoke, is not observed in the hookah water to a very high degree. Sugars and their decomposition products may be preferentially extracted from the smoke by the water, as they appear more concentrated in the spectrum of hookah water than of smoke. These experiments are exploratory in nature and should not be regarded as definitive, but they demonstrate that this technique could be used to investigate this system in detail. 


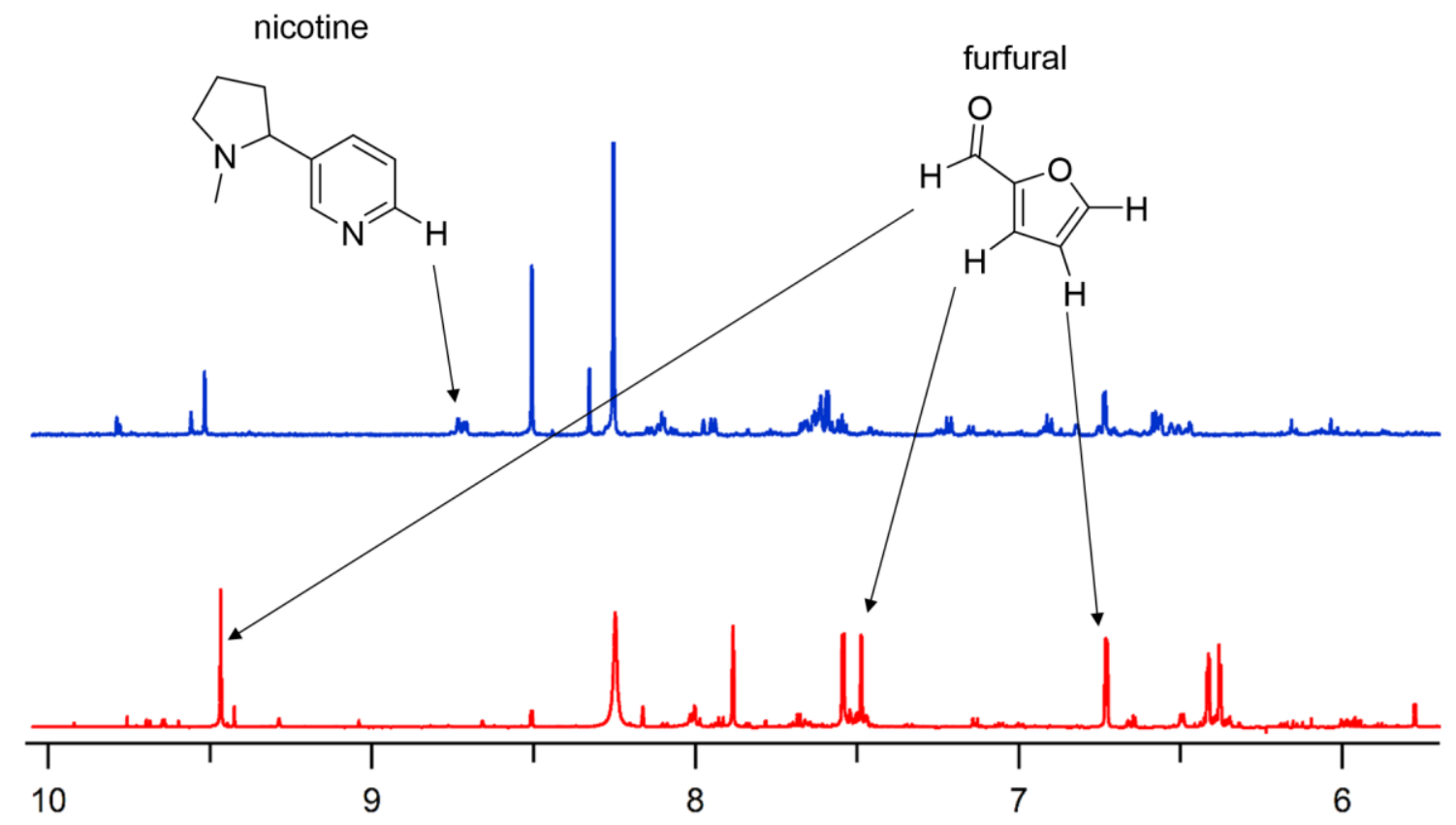

Figure 5.2 $-{ }^{1} \mathrm{H}$ NMR Spectra of Smoked Shisha and Water from the Hookah.

In Figure 5.2, plotted in red on bottom is a spectrum from hookah water through which shisha smoke has been pulled, while plotted in blue on top is a spectrum from a smoked shisha sample collected into $\mathrm{D}_{2} \mathrm{O}$ using a variation of the method developed for sampling e-liquids described in Chapter 3, Section 2. The smoked shisha contains different peaks associated with nicotine, flavorants, and decomposition products than are present in the hookah water, which contains large abundances of furfural and other sugar decomposition products.

\subsection{Summary}

Some types of shisha are very much like e-liquid in that they contain an abundance of glycerol and artificial flavorants. Compared to e-liquid, the addition of sugars and tobacco 
plant matter complicate the mixture of shisha somewhat. The method of smoking shisha is very different than e-liquid, utilizing smoldering charcoals to heat the mixture instead of resistively heated metal coils, but some of the same decomposition products can be observed in samples collected from both techniques. Glycerol and sugars, which are the most populous molecules in many shisha products, are susceptible to pyrolysis under the conditions of hookah smoking, and dehydration products such as glycidol and 5hydroxymethylfurfural can be observed in samples of shisha smoke. The water through which shisha smoke is pulled may selectively retain certain components of shisha smoke. 


\section{Conclusions and Future Work}

Glycerol and propylene glycol are susceptible to thermal decomposition by dehydration or oxidation. The temperature and extent of these degradation reactions depends on the heating surface and chemical composition of the samples; heating solutions containing dehydrating agents with a metal coil in an aerobic environment would almost certainly result in different chemistry than would occur from heating pure compounds with quartz under $100 \%$ helium atmosphere, as done in laboratory pyrolysis studies. Electronic cigarettes are capable of decomposing propylene glycol and glycerol into a diverse mixture of alcohols, alkenes, aldehydes, carboxylic acids, and epoxides. A few of these products have previously been discussed and detected as e-liquid decomposition products, but others have not been described in any reported e-cigarette studies. E-cigarettes are capable of vaporizing propylene glycol and glycerol without any evidence of decomposition when used conservatively. The surface of the heating coil is likely the most important single aspect in determining how much degradation occurs, which pathways dominate, and at what temperature decomposition begins.

Future work with an interest in the partial combustion and decomposition of glycerol and propylene glycol should focus on controlling the presence of oxygen in the atmosphere during sample collection, on directly measuring the temperature of the heating coil, on using isotopically-labeled propylene glycol and glycerol to understand the mechanisms and pathways leading to product formation, and on the continued identification of compounds present in vaporized samples. Future work with an interest in e-cigarettes should focus on 
developing methods to collect samples that have been inhaled into the lungs of a volunteer and exhaled into a perfluorinated bag, on determining the effect of nicotine and other compounds commonly used in e-liquids on the decomposition of propylene glycol and glycerol, and on the close analysis of heating coils for evidence of atypical surface features or anomalous composition that might be correlated with catalytic activity. 


\section{References}

(1) Glycerol; Miner, C. S., Dalton, N. N., Eds.; American Chemical Society Monograph Series; Reinhold Publishing Corporation: New York, 1953; Vol. 117.

(2) Glycols; Curme, Johnson, Eds.; American Chemical Society Monograph Series; Reinhold Publishing Corporation: New York, 1953; Vol. 114.

(3) Glycerol and the Glycols; Lawrie, J. W., Ed.; American Chemical Society Monograph Series; Chemical Catalog Company: New York, 1928; Vol. 44.

(4) Bamford, C. H.; Tipper, C. F. H. Comprehensive Chemical Kinetics: Oxidation and combustion reactions. v. 17. Gas-phase combustion; Elsevier Publishing Company, 1969.

(5) Hudlicky, M. Oxidations in Organic Chemistry; American Chemical Society Monograph Series; American Chemical Society: Washington DC, 1990; Vol. 186.

(6) The Pyrolysis of Carbon Compounds; Hurd, Ed.; American Chemical Society Monograph Series; Chemical Catalog Company: New York, 1929; Vol. 50.

(7) Cullis, C.; Newitt, E. The Royal Society, 1960; Vol. 257, pp 402-412.

(8) Scheele, C. W. Kongl Vetensk. Acad. Nya Handl. 1783, 4, 324.

(9) Scheele, C. W. Chem. Ann. Für Freunde Naturlehre Arzneygelahrtheit Haushaltungskunst Manufakturen 1784, 1, 99.

(10) Behrens, J. F. Dulcium Naturam Et Vires Expendens, University of Göttingen: Göttingen, Germany, 1779.

(11) Behrens, J. F. Chem. J. Für Freunde Naturlehre Arzneygelahrtheit Haushaltungskunst Manuf. 1780, 1780 (4), 187.

(12) Castle, T. Lexicon Pharmaceuticum, 2nd ed.; E. Cox: London, 1828.

(13) New Sydenham Society; Mayne, R. G.; Power, H.; Sedgwick, L. W. The New Sydenham Society's lexicon of medicine and the allied sciences: based on Mayne's Lexicon; London, 1879.

(14) Scheele, C. W. Chem. Ann. Für Freunde Naturlehre Arzneygelahrtheit Haushaltungskunst Manufakturen 1784, 2, 328.

(15) Asimov, I. Asimov On Chemistry, 2nd ed.; Anchor Books: Garden City, New York, 1975.

(16) Thorpe, T. E. Essays in Historical Chemistry; Macmillan: London, 1894.

(17) Wisniak, J. Rev. CENIC Cienc. Quím. 2009, 40 (3), 165.

(18) Wisniak, J. Educ. Quím. 2002, 13 (2), 133.

(19) Chevreul, M. E. Ann. Chim. 1813, 88, 225.

(20) Chevreul, M. E. Ann. Chim. 1815, 94, 80.

(21) Chevreul, M. E. Ann. Chim. 1815, 94, 113.

(22) Chevreul, M. E. Ann. Chim. 1815, 94, 225.

(23) Chevreul, M. E. Ann. Chim. 1815, 95, 5.

(24) Chevreul, M. E. Ann. Chim. 1816, 2, 339.

(25) Chevreul, M. E. Ann. Chim. Phys. 1817, 7, 155.

(26) Chevreul, M. E. Ann. Chim. Phys. 1817, 7, 264.

(27) Chevreul, M. E. Ann. Chim. Phys. 1817, 7, 367.

(28) Chevreul, M. E. Ann. Chim. 1815, 94, 73.

(29) Dijkstra, A. J. Ol. Corps Gras Lipides 2009, 16 (1), 8. 
(30) Chevreul, M. Recherches Chimiques sur les Corps Gras d'Origine Animale.; F. G. Levrault: Paris, 1823.

(31) Pelouze, J. Ann. Chim. Phys. 1836, 63, 19.

(32) Hess, G. H. Ann. Pharm. 1836, 20 (1), 9.

(33) Liebig, J. Ann. Chim. Phys. 1835, 59, 289.

(34) Liebig, J. Ann. Pharm. 1835, 14 (2), 133.

(35) Berzelius, J. J.; Wöhler, F.; Öngren, O. G. In Lehrbuch der Chemie; Lehrbuch der Chemie; Dresden and Leipzig, 1839; pp 657-669.

(36) Redtenbacher, J. Ann. Chem. Pharm. 1843, 47 (2), 113.

(37) Berthelot, P. E. M. Ann. Chim. Phys. 1854, 41, 216.

(38) Sobrero, A. Comptes Rendus 1847, 25, 247.

(39) Wurtz, C. A. Ann. Chim. Phys. 1857, 51, 94.

(40) Williamson, A. W. Proc. R. Soc. Lond. 1854, 7, 130.

(41) Wurtz, C. A. Ann. Chim. Phys. 1855, 43 (3), 492.

(42) Wisniak, J. Educ. Quím. 2011, 19 (1).

(43) Wurtz, C. A. Ann. Chim. Phys. 1859, 55 (3), 400.

(44) Nef, J. U. Justus Liebigs Ann. Chem. 1904, 335 (1-2), 191.

(45) Nef, J. U. Justus Liebigs Ann. Chem. 1904, 335 (3), 247.

(46) Harnitzky, T.; Menschutkine, N. Ann. Chem. Pharm. 1865, 136 (1), 126.

(47) Schulz, M.; Tollens, B. Justus Liebigs Ann. Chem. 1896, 289 (1), 20.

(48) Trillat, A.; Cambier, R. Comptes Rendus 1894, 118 (23), 1277.

(49) Sabatier, P.; Gaudin, G. Comptes Rendus 1918, 166, 1033.

(50) Katryniok, B.; Kimura, H.; Skrzyńska, E.; Girardon, J.-S.; Fongarland, P.; Capron, M.; Ducoulombier, R.; Mimura, N.; Paul, S.; Dumeignil, F. Green Chem. 2011, 13 (8), 1960.

(51) Katryniok, B.; Paul, S.; Dumeignil, F. ACS Catal. 2013, 3 (8), 1819.

(52) Katryniok, B.; Paul, S.; Bellière-Baca, V.; Rey, P.; Dumeignil, F. Green Chem. 2010, 12 (12), 2079.

(53) Ülgen, A. Conversion of glycerol to the valuable intermediates acrolein and allyl alcohol in the presence of heterogeneous catalysts, RWTH Aachen University, 2009.

(54) Tran, N. H.; Kannangara, G. S. K. Chem. Soc. Rev. 2013, 42 (24), 9454.

(55) Len, C.; Luque, R. Sustain. Chem. Process. 2014, 2 (1), 1.

(56) Zhou, C.-H. (Clayton); Beltramini, J. N.; Fan, Y.-X.; Lu, G. Q. (Max). Chem Soc Rev 2008, 37 (3), 527.

(57) Metzger, B. Glycerol Combustion, North Carolina State University, 2007.

(58) Bohon, M. D.; Metzger, B. A.; Linak, W. P.; King, C. J.; Roberts, W. L. Proc. Combust. Inst. 2011, 33 (2), 2717.

(59) Sheldon, R. A.; Kochi, J. K. Metal-catalyzed oxidations of organic compounds: mechanistic principles and synthetic methodology including biochemical processes; Academic Press: New York, 1981.

(60) Carrettin, S.; McMorn, P.; Johnston, P.; Griffin, K.; Kiely, C. J.; Hutchings, G. J. Phys. Chem. Chem. Phys. 2003, 5 (6), 1329. 
(61) Tsukuda, E.; Sato, S.; Takahashi, R.; Sodesawa, T. Catal. Commun. 2007, 8 (9), 1349.

(62) Colmenares, J. C.; Luque, R. Chem Soc Rev 2014, 43 (3), 765.

(63) Wang, F.; Dubois, J.-L.; Ueda, W. J. Catal. 2009, 268 (2), 260.

(64) Corma, A.; Huber, G. W.; Sauvanaud, L.; O’Connor, P. J. Catal. 2008, 257 (1), 163.

(65) Thompson, N. J. Ind. Eng. Chem. 1929, 21 (2), 133.

(66) Scott, G. S.; Jones, G. W.; Scott, F. E. Anal. Chem. 1948, 20 (3), 238.

(67) Laino, T.; Tuma, C.; Moor, P.; Martin, E.; Stolz, S.; Curioni, A. J. Phys. Chem. A 2012, 116 (18), 4602.

(68) Hemings, E. B.; Cavallotti, C.; Cuoci, A.; Faravelli, T.; Ranzi, E. Combust. Sci. Technol. 2012, 184 (7-8), 1164.

(69) Stein, Y. S.; Antal, M. J.; Jones, M. J. Anal. Appl. Pyrolysis 1983, 4 (4), 283.

(70) Philip Morris USA; Blake, C.; Posset, L.; Bösch, V.; Droz, A. The Effect of the Humectants Glycerol and Propylene Glycol on Mainstream and Sidestream Smoke Deliveries of Acrolein, Formaldehyde, Acetaldehyde, Acetone, and Propionaldehyde.; Philip Morris Europe, 1987.

(71) Philip Morris USA. Evaluation of Glycerol for Use as a Cigarette Ingredient; 2001.

(72) Philip Morris USA. Evaluation of Propylene Glycol for Use as a Cigarette Ingredient; 2001.

(73) Wertz, M. S.; Kyriss, T.; Paranjape, S.; Glantz, S. A. PLoS Med. 2011, 8 (12).

(74) Paine III, J. B.; Pithawalla, Y. B.; Naworal, J. D.; Thomas Jr., C. E. J. Anal. Appl. Pyrolysis 2007, 80 (2), 297.

(75) Laino, T.; Tuma, C.; Curioni, A.; Jochnowitz, E.; Stolz, S. J. Phys. Chem. A 2011, 115 (15), 3592.

(76) Rossiter, W. J.; Godette, M.; Brown, P. W.; Galuk, K. G. Sol. Energy Mater. 1985, $11(5), 455$.

(77) Díaz, E.; Sad, M. E.; Iglesia, E. ChemSusChem 2010, 3 (9), 1063.

(78) Grana, R.; Benowitz, N.; Glantz, S. A. Circulation 2014, 129 (19), 1972.

(79) Schroeder, M. J.; Hoffman, A. C. Tob. Control 2014, 23 (suppl 2), ii30.

(80) Electronic Cigarettes - An Overview; German Cancer Research Center, Series Ed.; Heidelberg, 2013.

(81) Electronic Cigarettes - An Overview - Supplement March 2014; German Cancer Research Center, Series Ed.; Heidelberg, 2014.

(82) Bell, K.; Stimson, G. V. Int. J. Drug Policy 2015, 26 (6), 533.

(83) Orellana-Barrios, M. A.; Payne, D.; Mulkey, Z.; Nugent, K. Am. J. Med. 2015, 128 (7), 674.

(84) Ebbert, J. O.; Agunwamba, A. A.; Rutten, L. J. Mayo Clin. Proc. 2015, 90 (1), 128.

(85) Caponnetto, P.; Saitta, D.; Sweanor, D.; Polosa, R. Int. J. Drug Policy 2015, 26 (6), 554.

(86) Evans, S. E.; Hoffman, A. C. Tob. Control 2014, 23 (suppl 2), ii23. 
(87) Farsalinos, K. E.; Romagna, G.; Tsiapras, D.; Kyrzopoulos, S.; Voudris, V. Int. J. Environ. Res. Public. Health 2013, 10 (6), 2500.

(88) Behar, R. Z.; Hua, M.; Talbot, P. PLOS ONE 2015, 10 (2), e0117222.

(89) Kosmider, L.; Sobczak, A.; Fik, M.; Knysak, J.; Zaciera, M.; Kurek, J.; Goniewicz, M. L. Nicotine Tob. Res. 2014, 16 (10), 1319.

(90) Goniewicz, M. L.; Knysak, J.; Gawron, M.; Kosmider, L.; Sobczak, A.; Kurek, J.; Prokopowicz, A.; Jablonska-Czapla, M.; Rosik-Dulewska, C.; Havel, C.; Jacob, P.; Benowitz, N. Tob. Control 2013, tobaccocontrol.

(91) Uchiyama, S.; Ohta, K.; Inaba, Y.; Kunugita, N. Anal. Sci. 2013, 29 (12), 1219.

(92) Jo, S.-H.; Kim, K.-H. J. Chromatogr. A 2016, 1429, 369.

(93) Geiss, O.; Bianchi, I.; Barrero-Moreno, J. Int. J. Hyg. Environ. Health 2016, 219 (3), 268.

(94) Gillman, I. G.; Kistler, K. A.; Stewart, E. W.; Paolantonio, A. R. Regul. Toxicol. Pharmacol. 2016, 75, 58.

(95) Flora, J. W.; Meruva, N.; Huang, C. B.; Wilkinson, C. T.; Ballentine, R.; Smith, D. C.; Werley, M. S.; McKinney, W. J. Regul. Toxicol. Pharmacol. 2016, 74, 1.

(96) Williams, M.; Villarreal, A.; Bozhilov, K.; Lin, S.; Talbot, P. PLOS ONE 2013, 8 (3), e57987.

(97) Goel, R.; Durand, E.; Trushin, N.; Prokopczyk, B.; Foulds, J.; Elias, R. J.; Richie, J. P. Chem. Res. Toxicol. 2015, 28 (9), 1675.

(98) Hahn, J.; Monakhova, Y. B.; Hengen, J.; Kohl-Himmelseher, M.; Schüssler, J.; Hahn, H.; Kuballa, T.; Lachenmeier, D. W. Tob. Induc. Dis. 2014, 12, 23.

(99) Nimlos, M. R.; Blanksby, S. J.; Qian, X.; Himmel, M. E.; Johnson, D. K. J. Phys. Chem. A 2006, 110 (18), 6145.

(100) Arceo, E.; Marsden, P.; Bergman, R. G.; Ellman, J. A. Chem. Commun. 2009, No. 23, 3357.

(101) Dai, Z.; Hatano, B.; Tagaya, H. Appl. Catal. Gen. 2004, 258 (2), 189.

(102) Bergens, S. H.; Bosnich, B. J. Am. Chem. Soc. 1991, 113 (3), 958.

(103) Walker, J. F. Formaldehyde, 3rd ed.; American Chemical Society Monograph Series; Robert E. Krieger Publishing Company: New York, 1975; Vol. 159.

(104) Townsend, D.; Lahankar, S. A.; Lee, S. K.; Chambreau, S. D.; Suits, A. G.; Zhang, X.; Rheinecker, J.; Harding, L. B.; Bowman, J. M. Science 2004, 306 (5699), 1158.

(105) Elango, M.; Parthasarathi, R.; Subramanian, V.; Sarkar, U.; Chattaraj, P. K. J. Mol. Struct. THEOCHEM 2005, 723 (1-3), 43.

(106) Dias, V.; Duynslaegher, C.; Contino, F.; Vandooren, J.; Jeanmart, H. Combust. Flame 2012, 159 (5), 1814.

(107) Butlerov, A. M. Ann. Chem. Pharm. 1859, 111, 242.

(108) Hofmann, A. W. Justus Liebigs Ann. Chem. 1868, 145 (3), 357.

(109) Butlerov, A. M. Comptes Rendus 1861, 53, 145.

(110) Breslow, R. Tetrahedron Lett. 1959, 1 (21), 22.

(111) Schwartz, A. W.; Graaf, R. M. J. Mol. Evol. 36 (2), 101.

(112) Jensen, R. P.; Luo, W.; Pankow, J. F.; Strongin, R. M.; Peyton, D. H. N. Engl. J. Med. 2015, 372 (4), 392. 
(113) Silverman, D. C.; Freeman, J. J. Ind. Eng. Chem. Process Des. Dev. 1983, 22 (3), 441.

(114) Johnson, R. A.; Stanley, A. E. Appl. Spectrosc. 1991, 45 (2), 218.

(115) Johnson, R. A.; Army, T. U. S. O. A. A. R. B. T. S. O. T. Method for obtaining the spectra of an unstable product; 1990.

(116) Fromme, H.; Schober, W. Atmos. Environ. 2015, 106, 429.

(117) Pepper, J. K.; Eissenberg, T. Chem. Res. Toxicol. 2014, 27 (8), 1336. 